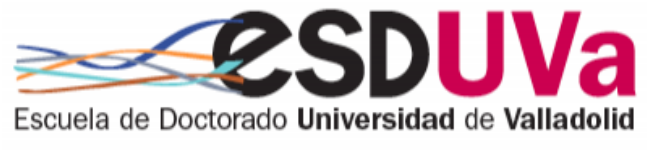

Universidad deValladolid

PROGRAMA DE DOCTORADO EN CIENCIA E INGENIERÍA AGRÍCOLA Y DE BIOSISTEMAS

TESIS DOCTORAL:

\title{
CARACTERIZACIÓN FISICO QUÍMICA Y APROVECHAMIENTO DE DOS ESPECIES PIRÓFILAS MEDITERRÁNEAS: CISTUS LADANIFER L. Y ERICA ARBOREA L.
}

\author{
Presentada por Paula Carrión Prieto \\ para optar al Grado de Doctor \\ por la Universidad de Valladolid
}

Dirigida por:

Dr. Salvador Hernández Navarro

Dr. Pablo Martín Ramos 



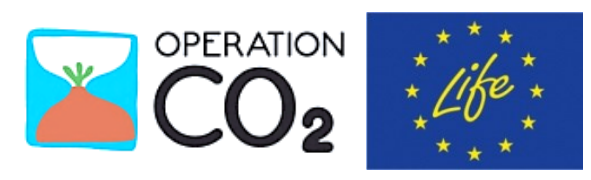

La presente Tesis Doctoral ha sido desarrollada dentro del marco del proyecto europeo LIFE+ “Operation $\mathrm{CO}_{2}$ ” (LIFE11 ENV/ES/535).

El objetivo principal del mismo es demostrar la viabilidad de los proyectos forestales y agroforestales en el secuestro de carbono en Europa. En este sentido, la primera línea de investigación se centra en el papel que representan como sumidero de $\mathrm{CO}_{2}$ dos especies de matorral en una zona del proyecto.

Por otro lado, busca contribuir de manera significativa en la lucha contra el cambio climático y conseguir la conservación y el manejo de bosques y carbono. Para ello, es imprescindible conocer el potencial económico y ecológico de las especies, por lo que siguiendo esta orientación surgen el resto de líneas de estudio, para la caracterización físico química de ambas especies, con el fin de enmarcar, reconocer y valorizar su composición como materia prima y un óptimo aprovechamiento de la vegetación mediterránea. 

"Porque eso es, en verdad, toda investigación: un hozar permanente entre la porquería y la quincalla para después, con seso y paciencia, llevar finalmente algo de sentido al papel en blanco. Algo que, además, sostenga sin desmayo la locura de la búsqueda, el caos del acopio, el placer del hallazgo, el sufrir de la reflexión y, finalmente (como espero), el premio del colofón"

Ramón Acín. La Noche antes de irse, 2016 



\section{AGRADECIMIENTOS}

A los Profesores Salvador Hernández Navarro y Pablo Martín Ramos, por aceptar la tutoría de la presente Tesis y dirigir su realización.

Al Profesor Jesús Martín Gil, promotor de las investigaciones, por su asesoramiento continuo en la aplicación de métodos espectroscópicos y por su aliento hasta el final. A la Profesora Mercedes Sánchez Báscones, por poner siempre su laboratorio y sus equipos a mi disposición.

Al Profesor Ioannis A. Barboutis, por su acogida en la Universidad Aristóteles de Tesalónica, a D. Xristos Damianidis por su ayuda inestimable en la toma de muestras y a la Dra. Vasiliki Kamperidou por su colaboración y amistad.

Al Profesor Jordi Voltas Velasco de la Universitat de Lleida y a la Profesora Anastasia A. Knorre de la Universidad Federal de Siberia, quienes a la altura de 2009 me iniciaron en la metodología investigadora.

Al Profesor Zacarías Clérigo Pérez, por incluir las investigaciones dentro del Proyecto LIFE. No en menor medida, al Departamento de Ingeniería Agrícola y Forestal y al Laboratorio de Técnicas Instrumentales de la UVa, por proporcionar la infraestructura material para realizar el trabajo.

A D. Eliecer Herrero Llorente por su ayuda en campo y por su buen humor. Al Dr. Luis Fernando Sánchez Sastre, por su apoyo, por ser mi referente. Al Dr. Norlan Miguel Ruiz Postome, por sus ánimos y los cafés cargados de humor negro que sólo los días grises pueden mejorar. A la Dra. Carmen Bravo Sánchez, por su interés en ayudarme. El palacio habría sido un pajar sin vosotros. A la Dra. Petruta Mihaela Matei, por las horas de laboratorio compartidas y conversaciones entre microondas.

A mis amigas Laura Misiego Ferrero, Elena Manuel Martín y Berta Martínez Iglesias, por ser incondicionales. Al Quevedo de este siglo, Arturo García Pulido, por tener siempre el comentario perfecto. A Patricia Valero Torrijos, Silvia Peña Lozano, José Alberto Pérez Castellano y el resto de voluntarios. A mis compañeros de carreras y entrenamientos, María Villalba Escudero, Miguel Sirgo del Valle, Rubén Gil Alfageme. A Gonzalo Arranz Montero, por su auxilio en momentos críticos. A Jesús Merino Pérez, por recordarme el mérito del día a día y del esprint final. A los demás compañeros del C.D. Triatlón Pisuerga.

Y por último, agradecer a los que estuvieron primero, a mi familia. A mi madre, Belén Prieto Sainz, porque cada palabra mía le pertenece, cada título, todo. A mi padre, Francisco Carrión García, porque soy su vivo reflejo. A mi hermano Francisco, por ser la única persona capaz de sacar lo mejor y lo peor de mí. A Encar Prieto Sainz y a David Zaraín. A mis tías, tíos y primos. A mis abuelos, Francisco Carrión de la Cal y Ma Luisa García Domínguez. A mi abuela, Carmen Sainz Zaraín. 

TABLA DE CONTENIDOS 



\section{TABLA DE CONTENIDOS}

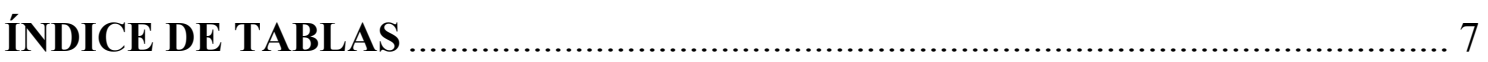

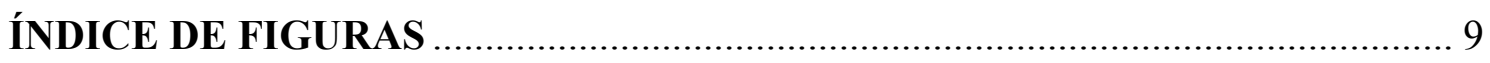

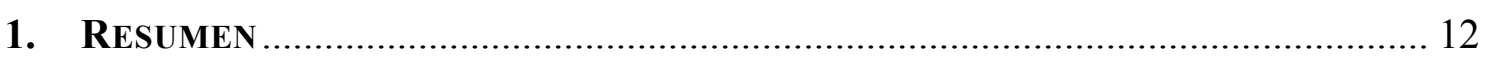

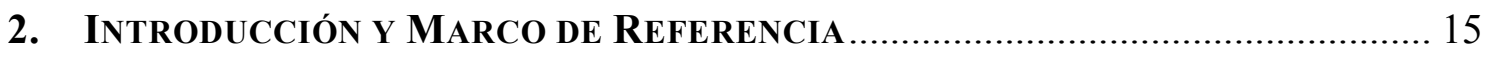

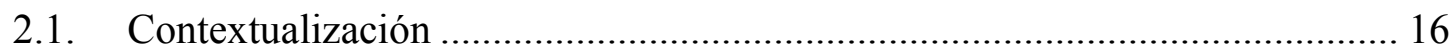

2.1.1. El fenómeno de la matorralización en la región mediterránea. Idoneidad del estudio de los matorrales de nuestra área geográfica. ..................................... 16

2.1.2. La biomasa forestal en el escenario energético del PER 2011-2020........ 17

2.2. CARACTERIZACIÓN DE JARALES Y BREZALES. SISTEMÁTICA, FILOGEOGRAFÍA Y USOS DE LAS ESPECIES ARBUSTIVAS BAJO ESTUDIO ................................................. 22

2.2.1. Características generales de los jarales. Morfología, distribución y usos de

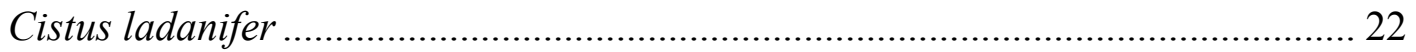

2.2.2. Características generales de los brezales. Morfología, distribución y usos de Erica arborea .............................................................................................. 26

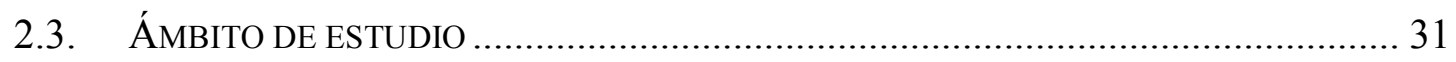

2.3.1. Situación administrativa y económica .................................................. 31

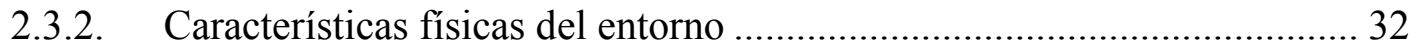

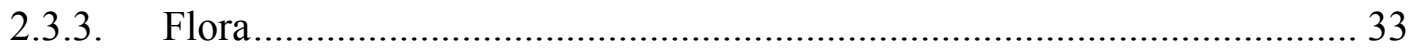

2.4. MARCO DE REFERENCIA. ESTADO DEL CONOCIMIENTO SOBRE LAS CARACTERÍSTICAS Y APROVECHAMIENTO DE LAS ESPECIES ARBUSTIVAS OBJETO DE

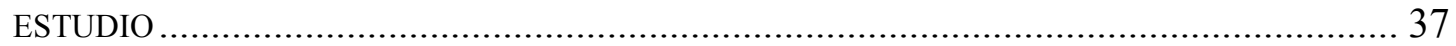

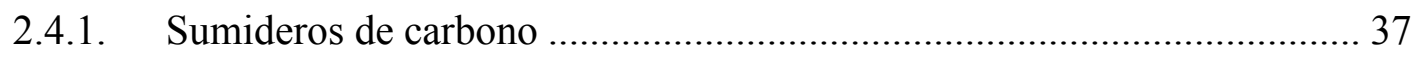

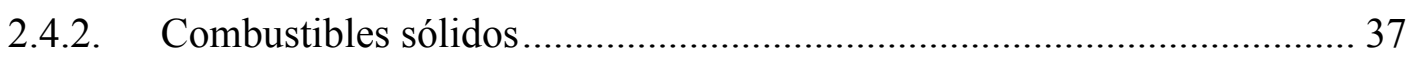

2.4.3. Precursores de productos de alto valor añadido en Medicina $y$

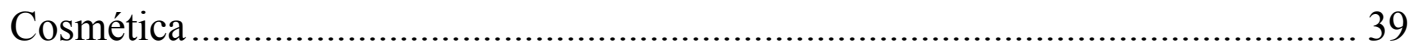

2.4.4. Precursores de combustibles líquidos: furfural e hidroximetilfurfural..... 41

2.4.5. Precursores para materiales compuestos: microfibras de celulosa como

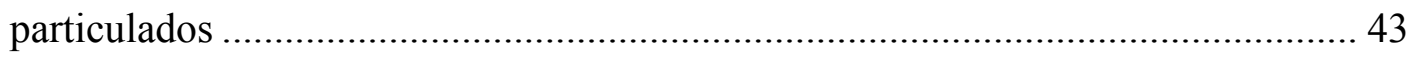

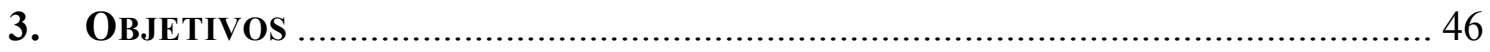

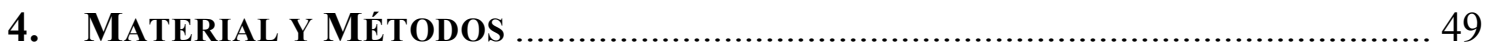

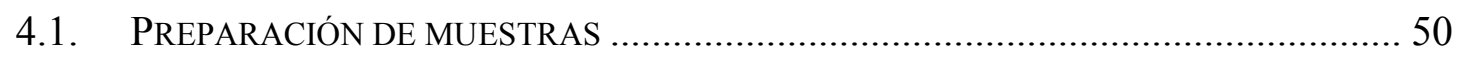


4.2. METOdOlogía PARA EL ANÁlisis DEL MATORRAL MEDITERRÁNeO COMO SUMIDEROS DE CARBONO Y PARA EL CÁLCULO DE NUEVAS RELACIONES ROOT-TOSHOOT

4.3. METODOLOGÍA PARA LA VALORIZACIÓN DE CISTUS LADANIFER Y ERICA ARBOREA COMO COMBUSTIBLES SÓLIDOS: CARACTERIZACIÓN TÉRMICA DE MADERA Y CORTEZA 54

4.4. MetOdología PARA El ESTUdio VIBRACIONAL Y TÉRMICO DE LOS ACEITES ESENCIALES DE CISTUS LADANIFER Y ERICA ARBOREA 56

4.5. METOdología PARA EL FRACCIONAMiENTO DE LA BiOMASA DE Cistus LADANIFER Y ERICA ARBOREA Y SU CONVERSIÓN A PRODUCTOS CON VALOR AÑADIDO. CARACTERIZACIÓN DE AZÚCARES, LIGNINA, FURFURAL, 5-HIDROXIMETIL FURFURAL Y MICROFIBRAS CRISTALINAS DE CELULOSA

4.5.1. Métodos de determinación de azúcares, lignina, furfural e hidroximetilfurfural

4.5.2. Tratamiento de cristalinización de la celulosa y medidas de accesibilidad y cristalinidad 61

5. Resultados 63

5.1. Publicación \#1: MEDITERRANEAN SHRUBLANDS AS CARBON SINKS FOR Climate Change Mitigation: NEW ROOT TO SHOOT RATIOS 64

5.2. Publicación \#2: VALORIZATION OF CISTUS LADANIFER AND ERICA ARBOREA SHRUBS FOR FUEL: WOOD AND BARK THERMAL CHARACTERIZATION 83

5.3. Publicación \#3: Vibrational and Thermal Studies of Essential Oils DERIVED FROM CISTUS LADANIFER AND ERICA ARBOREA SHRUBS 96

5.4. Publicación \#4: ON THE CRystallinity of Cellulose MicrofiBers DERIVED FROM C. LADANIFER AND E. ARBOREA SHRUBS 102

5.5. Publicación \#5: Furfural, 5-HMF, ACID-SOLUble LIGNIN AND NONREDUCING SUGAR CONTENTS IN C. LADANIFER AND E. ARBOREA LIGNOCELLULOSIC BIOMASS HYDROLYSATES 1133

6. Conclusiones 127

7. Bibliografía 134 


\section{ÍNDICE DE TABLAS}

Tabla 1. Objetivos 2010, 2015 y 2020 del plan de energías renovables 2011-2020 en el sector eléctrico (potencia instalada, generación bruta sin normalizar y generación bruta normalizada). Datos IDAE 18

Tabla 2. Objetivos 2010, 2015 y 2020 del plan de energías renovables 2011-2020 en el sector de la calefacción y la refrigeración. Datos IDAE 18

Tabla 3. (Publicación 1, Tabla 1) Main absorption bands in the FTIR spectra of the different fractions of $C$. ladanifer and E. arborea and their assignments according to the literature (Emmanuel, Odile, and Céline 2015, Faust 1997, Schwanninger et al. 2004, Traoré, Kaal, and Martínez Cortizas 2016).... 78

Tabla 4. (Publicación 1, Tabla 2) Corrected areas values for each fraction in C. ladanifer and E. arborea.

Tabla 5. (Publicación 1, Tabla 3) Percentages in terms of vegetal components for C. ladanifer and E. arborea (Ferro et al. 2015, Barboni et al. 2010, Leroy, Leoni, and Cancellieri 2010). 79

Tabla 6. (Publicación 1, Tabla 4) Comparison of root-to-shoot ratios for C. ladanifer and $E$. arborea determined by two methodologies: using vibrational data $\left(R_{\mathrm{FTIR}}\right)$ and using the UN-ECE/FAO-IPCC procedure modified by Sanquetta, Corte, and da Silva (2011) $\left(R_{\text {biomass }}\right)$......

Tabla 7. (Publicación 1, Tabla 5) C content, $\mathrm{N}$ content and C:N ratios for C. ladanifer and E. arborea.

Tabla 8. (Publicación 1, Tabla 6) Estimated biomass (dry matter), carbon stock and carbon dioxide equivalent.

Tabla 9. (Publicación 2, Tabla 1) Stem diameter and bark thickness of the shrub species under study. Values are given as an average of 10 repetitions, followed by the the minimum and maximum values in brackets.

Tabla 10. (Publicación 2, Tabla 2) Overall chemical composition of $C$. ladanifer and $E$. arborea (Carrión-Prieto et al. 2017). Values are given as an average of 25 repetitions, followed by the minimum and maximum values in brackets.

Tabla 11. (Publicación 2, Tabla 3) Carbon $(\mathrm{C})$, hydrogen $(\mathrm{H})$, nitrogen $(\mathrm{N})$ and oxygen (O) percentages for C. ladanifer and E. arborea fractions and HHV values calculated thereof. 
Tabla 13. (Publicación 2, Tabla 4) Percentage of biomass distribution in each plant (Carrión-Prieto et al. 2017)

Tabla 14. (Publicación 2, Tabla 5) Exothermic effects data for holocellulose and lignin in the DSC themograms for C. ladanifer and E. arborea woods

Tabla 15. (Publicación 2, Tabla 6) Experimental values for ash content (AC) from bark and wood fractions

Tabla 16. (Publicación 2, Tabla 7) Minimum barked diameter to meet the requirements of ISO 17225-2 norm (ISO 2014) for ash content of pellets and associated HHV values

Tabla 17. (Publicación 3, Tabla 1) Main bands in the ATR-FTIR vibrational spectra of C. ladanifer and E. arborea oils and various plant components. All wavenumber values are in $\mathrm{cm}^{-1}$. 98

Tabla 18. (Publicación 3, Tabla 2) Comparison of the vibrational spectra of C. ladanider and E. arborea oils with rosehip and palm oils, myrrh and mastic resins and tragacanth gum. 98

Tabla 19. (Publicación 4, Tabla 1) ISV Values and Accessibility and Crystallinity Estimations for Treated Cellulose Fibers from Different Origins 108

Tabla 20. (Publicación 5, Tabla 1) Lignin, furfural and 5-HMF in hydrolysates after a deep eutectic solvent MW-assisted extraction. 118

Tabla 21. (Publicación 5, Tabla 2) Comparative measurements of soluble lignin, furfural and HMF in the hydrolysates.

Tabla 22. (Publicación 5, Tabla 3) Total (TS), reducing (RS) and non-reducing (NRS) sugars concentrations for the $\mathrm{MW}$-assisted $\mathrm{ChCl} /$ urea $\mathrm{DMAc} / \mathrm{NaHCO}_{3}$ and DMAc/ $\mathrm{CH}_{3} \mathrm{OK}$ treatments as a function of exposure times. 122

Tabla 23. (Publicación 5, Tabla 4) Comparison of the sugar concentrations in the shrubs hydrolysates studied herein with values reported by other authors.

Tabla 24. (Publicación 5, Tabla 5) Kinetic coefficients (k), correlation coefficients $\left(\mathrm{r}^{2}\right)$ and initial concentration of each sample $\left(H_{o}\right)$ determined from the concentration as a function of time for lignin, furfural, 5-HMF, total sugars and reducing sugars production from the hydrolysis of E. arborea and C. ladanifer lignocellulosic biomass. 123 


\section{ÍNDICE DE FIGURAS}

Figura 1. Ejemplar de C. ladanifer con una rama de E. arborea en primer plano y un enfoque selectivo de cápsulas de jara. (Tomado de M. Casares, 2017)

Figura 2. Mapa de distribución de Cistus ladanifer en la Península Ibérica (haplotipos).

Figura 3. Jaral de Cistus ladanifer en tierras zamoranas 25

Figura 4. Mapa de distribución mundial de Erica arborea (imagen tomada de Discover Life http://www.discoverlife.org/mp/20q?search=Erica+arborea)

Figura 5. Ejemplar de E. arborea y un enfoque selectivo de un ramillete en flor 28

Figura 6. Brezal de Erica arborea en tierras zamoranas 29

Figura 7. Localización de la zona de estudio: Congosta, Ayoó de Vidriales, Zamora, España 31

Figura 8. De izquierda a derecha y de arriba abajo: Yema y ramilla de Pinus pinaster, hoja de Quercus pyrenaica, envés foliar de $Q$. ilex subsp. ballota, cápsulas y planta de Cistus psilosepalus, Halimium atriplicifolium, Halimium sp., Genista tridentata, Rosa sp. y muestra de Thymus sp. 35

Figura 9. Muestra de jara tras su extracción y proceso de clasificación de las fracciones.

Figura 10. Submuestras seleccionadas antes y después de la pulverización. 51

Figura 11. Lámina de estaño en balanza de precisión y equipo LECO CHN-2000 ...... 52

Figura 12. Preparación de una muestra en mortero tras su pesado (extremo superior izquierda), ejemplo de pastilla de muestra $\mathrm{KBr}$ (izquierda inferior), prensa utilizada en la formación de los pellets y equipo utilizado.

Figura 13. Proceso seguido en la determinación del contenido de cenizas, siguiendo la metodología descrita en ISO 18122:2015. En el extremo superior izquierdo, un fragmento del tallo previo al serrado, a su derecha, una muestra de los discos obtenidos; en el extremo inferior izquierdo, crisoles en mufla y a su derecha, en el desecador...... 55

Figura 14. Muestra de la exudación de C. ladanifer en campo 56

Figura 15. Secuencia del tratamiento con $\mathrm{NaOH}$ : pesado de muestras, adición de la base, proceso de agitación con la ayuda de imanes y resultado tras la digestión 57 
Figura 16. DES a base de cloruro de colina y urea donde el cation [colina] ${ }^{+}$es energéticamente energético con $\left[\mathrm{Cl}(\text { urea })_{2}\right]^{-}$

Figura 17. DES cristalizado a temperatura ambiente y preparación de muestras en horno microondas

Figura 18. Extracto de la disolución en $\mathrm{NaOH}$ en matraces para su registro espectrofotomético (imagen superior); distintas coloraciones tras los métodos descritos, siendo Miller, Dubois et al. y Chi et al. de izquierda a derecha, respectivamente (imágenes inferiores).

Figura 19. (Publicación 1, Figura 1). Cistus ladanifer L., also known as gum rockrose or labdanum (left); Erica arborea L., also known as briar root or tree heath (right)..... 80

Figura 20. (Publicación 1, Figura 2). Location of Castilla y León region in the Iberian Peninsula (left); location of Zamora province in Castilla y León region (center); location of the shrubland under study in Zamora province (right) 80

Figura 21. (Publicación 1, Figura 3). FTIR spectrum of E. arborea leaves with the corrected peak areas calculated at some selected wavenumbers $\left(\mathrm{cm}^{-1}\right)$. 80

Figura 22. (Publicación 1, Figura 4). Biomass distribution for Cistus ladanifer L. and Erica arborea L.

Figura 23. (Publicación 1, Figura 5). FTIR spectra of the different fractions (leaves, thin branches, thick branches and roots) of (a) C. ladanifer and (b) E. arborea. 81

Figura 24. (Publicación 1, Figura 6). Contribution of the different components to the total carbon and nitrogen stocks, for $C$. ladanifer and $E$. arborea, taking into consideration the biomass distribution.

Figura 25. (Publicación 2, Figura 1). DSC and TG/DTG curves of (a) C. ladanifer and (b) E. arborea woods.

Figura 26. (Publicación 2, Figura 2). DTA and TG/DTG curves of C. ladanifer stems: (a) external fraction and (b) internal fraction.

Figura 27. (Publicación 2, Figura 3). DTA and TG/DTG curves of C. ladanifer roots: (a) epidermis and (b) cortex

Figura 28. (Publicación 2, Figura 4). DTA and TG/DTG curves of E. arborea stems: (a) external fraction and (b) internal fraction.

Figura 29. (Publicación 2, Figura 5). DTA and TG/DTG curves of E. arborea roots: (a) epidermis and (b) cortex. 
Figura 30. (Publicación 3, Figura 1). FTIR spectra of (a) Cistus ladanifer oil and (b) Erica arborea oil.

Figura 31. (Publicación 3, Figura 2). Thermograms for (a) C. ladanifer oil, $\mathrm{m}=21.14$ $\mathrm{mg}$; and (b) E. arborea oil, $\mathrm{m}=17.77 \mathrm{mg}$. The left y axis corresponds to the DSC curve, while the two y axes on the right correspond to TG/DTG curves.

Figura 32. (Publicación 3, Figura 3). Low-temperature DSC thermograms for (a) C. ladanifer oil, $\mathrm{m}=4.1 \mathrm{mg}$; and (b) E. arborea oil, $\mathrm{m}=5.7 \mathrm{mg}$. The cooling/heating rate was $|\beta|=50^{\circ} \mathrm{C} \cdot \mathrm{min}^{-1}$.

Figura 33. (Publicación 4, Figura 1). X-ray powder diffraction patterns for the cellulose fibers from (a) E. arborea and (b) C. ladanifer after alkaline treatment. 106

Figura 34. (Publicación 4, Figura 2). X-ray powder diffraction patterns for the cellulose fibers from (a) E. arborea and (b) C. ladanifer after microwave-assisted DES treatment for $30 \mathrm{~min}$. The very sharp peaks observed in both diffractograms for $2 \theta>25^{\circ}$ are spurious and can be unequivocally ascribed to some remaining $\mathrm{TiO}_{2}$, anatase variety (ICDD PDF \# 04-002-8296), used in the treatment. 106

Figura 35. (Publicación 4, Figura 3). ATR-FTIR spectra of the cellulose fibers from E. arborea (solid line) and C. ladanifer (dashed line) after microwave-assisted DES treatment of the inner stem.

Figura 36. (Publicación 4, Figura 4). SEM micrographs of the fibers after microwaveassisted DES-based treatment: inner stem of C. ladanifer [400× (a) and $1000 \times$ (b) magnification] and inner stem of E. arborea [500× (c) and $1400 \times$ (d) magnification].

Figura 37. (Publicación 5, Figura 1). DES of $\mathrm{ChCl}$ and urea where a [choline $]^{+}$cation is energetically competitive with $\left[\mathrm{Cl}(\text { urea })_{2}\right]^{-}$. 116

Figura 38. (Publicación 5, Figura 2). Calibration curves for furfural, acid-soluble lignin and 5-(hydroxymethyl)-furfural concentration. 118

Figura 39. (Publicación 5, Figura 3). Lignin, furfural and HMF yields for the $E$. arborea and $C$. ladanifer lignocellulosic biomass hydrolysates after: (a) a deep eutectic solvent $\mathrm{MW}$-assisted extraction; (b) a polar aprotic solvent MW-assisted extraction; and (c) a DMAc/ $\mathrm{CH}_{3} \mathrm{OK}$ solvent $\mathrm{MW}$-assisted extraction. Total, reducing and no reducing sugars in the hydrolysates after: (d) a $\mathrm{MW}$-assisted $\mathrm{ChCl} /$ urea treatment; (e) a MW-assisted $\mathrm{DMAc} / \mathrm{NaHCO}_{3}$ treatment; and (f) a $\mathrm{MW}$-assisted DMAc $/ \mathrm{CH}_{3} \mathrm{OK}$ treatment. 119

Figura 40. (Publicación 5, Figura 4). Calibration curves for glucose concentration... 121

Figura 41. (Publicación 5, Figura 5). Proposed interaction between $\mathrm{DMAc}-\mathrm{NaHCO}_{3}$ and DMAc- $\mathrm{CH}_{3} \mathrm{OK}$ solvents and sugar polymer 



\section{RESUMEN}





\section{RESUMEN}

Cistus ladanifer L. y Erica arborea L. son los representantes más importantes de los ecosistemas de matorral de clima mediterráneo en nuestra área geográfica, donde ocupan grandes superficies vegetales. Su presencia en estas superficies ha venido siendo justificada por sus ventajas para reconstruir ecosistemas tras incendios forestales gracias a la adaptación de sus raíces (rebrotadoras obligadas). Recientemente, han comenzado a ser revalorizadas por su carácter de "sumideros perdidos" de carbono, necesarios para completar el efecto sumidero de su alternativa, los sistemas arbolados, y con ello cerrar el balance global de carbono a escala regional. Asimismo, el hecho de que ambas especies ardan con más facilidad que otras especies rebrotadoras debido a la presencia de aceites esenciales, ha determinado que fijemos nuestra atención en los componentes responsables de tales aceites, a efectos de caracterización. Finalmente, las aplicaciones como combustibles de los componentes de ambos arbustos, la explotación de sus aceites como productos de alto valor añadido para la industria de cosméticos y la valorización de furfural, hidroximetilfurfural y fibras de celulosa cristalina obtenidas del fraccionamiento y tratamiento de sus maderas son objeto de consideración.

Constituyen aportaciones sustanciales de la parte experimental de la Memoria: (a) el análisis cartográfico de C. ladanifer y E. arborea en un área de Castilla y León; (b) el análisis elemental, espectroscópico y termogravimétrico de raíz, tallo, hojas y aceites de ambas especies; (c) la cuantificación de biomasa, carbono y dióxido de carbono fijado por ambas especies; (d) la puesta a punto de un nuevo método para la obtención de cocientes root-to-shoot; (e) la determinación de los poderes caloríficos y cenizas de sus diversos componentes con vista a su aplicación como combustibles; (f) la caracterización de los terpenoides de sus aceites esenciales; $(g)$ la determinación de los contenidos de lignina, furfural, hidroximetilfurfural y azúcares reductores en hidrolizados; y $(h)$ la caracterización de la cristalinidad de las microfibras de celulosa para su aplicación potencial en materiales compuestos.

Palabras clave: Cistus ladanifer; Erica arborea; cocientes root-to-shoot; poderes caloríficos; terpenoides; aceites esenciales; furfural; hidroximetilfurfural. 



\begin{abstract}
Cistus ladanifer and Erica arborea are the most important representatives of Mediterranean climate scrub ecosystems in our geographical area, where they occupy large plant surfaces. Their presence in these areas can be ascribed to their advantages for the rebuilding of ecosystems after forest fires, due to the adaptation of their roots (obligate resprouting). They have recently begun to be revalued because of their role as carbon sinks, needed to complete the sink effect of their alternative, tree systems, and thereby to close the global carbon balance on a regional scale. Further, the fact that both species burn more easily than other resprouting species on account of the presence of essential oils, has led us to focus our attention on the components present in such oils, for characterization purposes. Finally, the applications as fuels of both shrubs, the exploitation of their oils as high added-value products for the cosmetics industry and the valorization of furfural, hydroxymethyl furfural and crystalline cellulose fibers obtained from the fractionation and treatment of their wood have been taken under consideration.

Substantive contributions of the experimental part of this $\mathrm{PhD}$ Thesis are: (a) the cartographic analysis of $C$. ladanifer and E. arborea distribution in an area of Castilla y León; (b) the elemental, spectroscopic and thermogravimetric analyses of root, stem, leaves and oils of both species; (c) the accurate quantification of biomass, carbon and carbon dioxide fixed by both species; $(d)$ the development of a new method to obtain root-to-shoot ratios; (e) the determination of higher heating values and ashes of their various components with a view to their application as fuels; $(f)$ the characterization of the terpenoids of their essential oils; $(g)$ the determination of furfural, hydroxymethylfurfural and reducing sugars in their derived hydrolysates; and $(h)$ the characterization of the crystallinity of the cellulose microfibers for their potential application in composite materials.
\end{abstract}

Keywords: Cistus ladanifer; Erica arborea; root-to-shoot ratios; higher heating values; essential oils; terpenoids; furfural; hydroxymethylfurfural; crystalline cellulose fibers. 

2.INTRODUCCIÓN Y MARCO DE REFERENCIA 



\section{INTRODUCCIÓN Y MARCO DE REFERENCIA}

\subsection{CONTEXTUALIZACIÓN}

\subsubsection{El fenómeno de la matorralización en la región mediterránea. Idoneidad del estudio de los matorrales de nuestra área geográfica.}

El reconocimiento del cambio climático como un problema real y el consiguiente establecimiento del Protocolo de Kyoto sirvieron como detonante en la investigación sobre el intercambio de carbono entre los ecosistemas terrestres y la atmósfera, siendo esta capacidad de asimilación el objetivo de las principales medidas de mitigación que se contemplan desde entonces. Sin embargo, se centran en los ecosistemas arbolados llevando a cabo reforestaciones y forestando terrenos agrícolas abandonados, principalmente-, y prescinden de ecosistemas no arbolados de gran abundancia como es el caso del matorral. Tanto es así, que FLUXNET, una red internacional que coordina observaciones micrometeorológicas regionales y mide los intercambios de dióxido de carbono, vapor de agua y energía entre los ecosistemas terrestres y la atmósfera, cuenta con poca información sobre los ecosistemas de matorral -los de menor representación a pesar de su gran extensión- y carece de estimaciones de su potencial de fijación de carbono (Baldocchi et al., 2001). Sin embargo, para estimar el ciclo de carbono a nivel regional es importante hacerlo también a escala ecosistema, describiendo detalladamente el ciclo de carbono con el fin de evitar "sumideros perdidos" que desajusten el balance global real de carbono (Kowalski, 2006). En el caso de los matorrales mediterráneos, la relevancia que ejercen en el funcionamiento y evolución de los ecosistemas terrestres es notable: restauración ecológica, protección hidrológica y edáfica, micorrizado. Además, el abandono de los usos tradicionales del suelo como la agricultura y ganadería hacen que la superficie de matorral sea cada vez mayor, conociéndose como procesos de matorralización. A pesar de que esta situación ha hecho aumentar la investigación, conociéndose con mayor detalle la composición de las formaciones de matorral mediterráneo, su cobertura y distribución, que por otro lado son aspectos necesarios para el diseño y validación de modelos y balances (no sólo para estimar el potencial de fijación de carbono sino también para conocer su viabilidad en otros usos y aprovechamientos), aún es necesario profundizar en su estructura y funcionamiento (Kummerow, 1989, Fernández et al., 1995). 
En España, Montero et al. $(2005 a ; 2005 b)$ propusieron una metodología de estimación de carbono para las principales especies forestales de carácter arbóreo, que consistía en inventariar el vuelo midiendo diámetros y alturas para obtener una estimación de la biomasa y el volumen de muestra por unidad de superficie; tras lo cual se calcula el contenido de carbono en hojas, tronco y raíces, determinando así las cantidades de carbono y de $\mathrm{CO}_{2}$ atmosférico equivalente. No obstante, no hay estudios similares sobre formaciones de matorral y las investigaciones que se desarrollan tienen como objetivo el estudio del potencial energético de la biomasa forestal, ya proceda de restos de podas y cortas o de procesos industriales de transformación de la madera (Centro de innovación y servicios de la madera, 2005).

\subsubsection{La biomasa forestal en el escenario energético del PER 2011-2020}

En la actualidad, la mayor parte de los $3.655 \mathrm{ktep}$ de consumo térmico final de biomasa en España proviene del sector forestal, utilizándose en sector doméstico mediante sistemas tradicionales poco eficientes (uso de leñas en equipos obsoletos) y en industrias forestales para consumo térmico o cogeneración. Existe una potencia instalada de 533 MW abastecida con residuos de industrias agroforestales y restos de cultivos agrícolas, principalmente (IDAE, 2011).

En los últimos años se está iniciando el desarrollo de los cultivos energéticos y de la mecanización específica para la recogida, extracción y tratamiento de la biomasa. Respecto a las aplicaciones, la implantación de tecnologías modernas para la biomasa térmica en edificios y los desarrollos tecnológicos en gasificación y ciclos ORC para la implantación de cogeneraciones hacen prever, para los próximos años, una importante expansión de la biomasa en el sector térmico en edificios e instalaciones industriales. Por consiguiente, además de avanzar en una mayor aportación cuantitativa por parte de la biomasa, se producirá un cambio cualitativo en cuanto a tecnologías actualizadas y eficientes (IDAE, 2011).

El potencial de biomasa disponible en España, bajo hipótesis conservadoras, se sitúa en torno a 88 millones de toneladas de biomasa primaria en verde, incluyendo restos de masas forestales existentes, restos agrícolas, masas existentes sin explotar y cultivos energéticos a implantar. A este potencial se suman más de 12 millones de toneladas de biomasa secundaria seca obtenida de residuos de industrias agroforestales (IDAE, 2011). 
Para alcanzar los objetivos fijados en el área de biomasa (Tablas 1 y 2) se han definido una serie de propuestas dirigidas a cada fase del aprovechamiento de la misma. Las propuestas para el desarrollo de un mercado maduro de suministro de biomasa se centran principalmente en la movilización del recurso. El apoyo al desarrollo de aplicaciones térmicas, especialmente en edificios, se realizará mediante campañas de difusión, desarrollos normativos y nuevos sistemas de apoyo financiero, de incentivos y de ayudas públicas a la inversión. El crecimiento de la producción eléctrica con biomasa se conseguirá mediante la generación y distribución a través de pequeñas cogeneraciones y centrales eléctricas en el entorno de $15 \mathrm{MW}$, para lo que se establecen nuevos programas de financiación y mejoras en el sistema de retribución de la energía eléctrica renovable (especialmente para instalaciones con menos de $2 \mathrm{MW}$ ).

Tabla 1. Objetivos 2010, 2015 y 2020 del plan de energías renovables 2011-2020 en el sector eléctrico (potencia instalada, generación bruta sin normalizar y generación bruta normalizada). Datos IDAE

\begin{tabular}{|c|c|c|c|c|c|c|c|c|c|}
\hline & \multicolumn{3}{|c|}{2010} & \multicolumn{3}{|c|}{2015} & \multicolumn{3}{|c|}{2020} \\
\hline & MW & GWh & GWh (*) & MW & GWh & GWh (*) & MW & GWh & GWh (*) \\
\hline Total & 825 & 4228 & $(* *)$ & 1162 & 7142 & $(* *)$ & 1950 & 12200 & $(* *)$ \\
\hline $\begin{array}{l}\text { Biomasa } \\
\text { sólida }\end{array}$ & 533 & 2820 & $(* *)$ & 817 & 4903 & $(* *)$ & 1350 & 8100 & $(* *)$ \\
\hline Residuos & 115 & 663 & $(* *)$ & 125 & 938 & $(* *)$ & 200 & 1500 & $(* *)$ \\
\hline Biogás & 177 & 745 & $(* *)$ & 220 & 1302 & $(* *)$ & 400 & 2600 & $(* *)$ \\
\hline
\end{tabular}

Tabla 2. Objetivos 2010, 2015 y 2020 del plan de energías renovables 2011-2020 en el sector de la calefacción y la refrigeración. Datos IDAE

\begin{tabular}{lcccccccccccc}
\hline Ktep & $\mathbf{2 0 0 5}$ & $\mathbf{2 0 1 0}$ & $\mathbf{2 0 1 1}$ & $\mathbf{2 0 1 2}$ & $\mathbf{2 0 1 3}$ & $\mathbf{2 0 1 4}$ & $\mathbf{2 0 1 5}$ & $\mathbf{2 0 1 6}$ & $\mathbf{2 0 1 7}$ & $\mathbf{2 0 1 8}$ & $\mathbf{2 0 1 9}$ & $\mathbf{2 0 2 0}$ \\
\hline Biomasa & 3468 & 3729 & 3779 & 3810 & 3851 & 3884 & 4060 & 4255 & 4377 & 4485 & 4542 & 4653 \\
Sólida & 3441 & 3695 & 3740 & 3765 & 3800 & 3827 & 3997 & 4185 & 4300 & 4400 & 4450 & 4553 \\
Biogás & 27 & 34 & 39 & 45 & 51 & 57 & 63 & 70 & 77 & 85 & 92 & 100
\end{tabular}

* La biomasa sólida incluye residuos

En el Plan de Implementación de la Industria Europea sobre Bioenergía (EIBI), por lo que respecta al ámbito de la biomasa sólida y gaseosa, se recoge la necesidad de avanzar en todos los aspectos referentes a la disponibilidad y logística de la biomasa como materia prima para usos energéticos, y se mencionan tres cadenas de valor por su especial interés, todas ellas basadas en procesos termoquímicos de conversión de la materia prima:

- Biometano y otros combustibles gaseosos a partir de biomasa vía gasificación. 
- Generación de energía eléctrica de alta eficiencia mediante gasificación de biomasa.

- Vectores bioenergéticos a partir de biomasa mediante procesos termoquímicos distintos a la gasificación.

A nivel nacional, la Plataforma Tecnológica Española de la Biomasa, el BIOPLAT, identifica los retos tecnológicos encuadrados en dos cadenas de valor: la utilización de biocombustibles sólidos mediante combustión directa y la producción y utilización de biocombustibles sólidos para gasificación.

El análisis de los últimos datos de consumo de biomasa térmica en España (3.655 kept) por Comunidades Autónomas permite advertir que Andalucía, Galicia y Castilla y León son las autonomías con mayor consumo. Dicho consumo superior está relacionado con la existencia de empresas que precisan gran cantidad de biomasa y con la presencia de un sector forestal desarrollado, además de por tener una estructura de población en la que el sector rural tiene un peso mayor y, por tanto, un consumo superior en el ámbito doméstico (IDAE, 2011).

El uso de la biomasa como recurso energético en lugar de los combustibles fósiles comúnmente utilizados supone unas ventajas medioambientales de primer orden, como son: ciclo neutro de $\mathrm{CO}_{2}$, sin contribución al efecto invernadero; disminución de emisiones de azufre; disminución de emisiones de partículas; emisiones reducidas de contaminantes como $\mathrm{CO}, \mathrm{HC}$ y $\mathrm{NO}_{\mathrm{x}}$; reducción del mantenimiento de los peligros derivados del escape de gases tóxicos y combustibles en las casas; reducción de riesgos de incendios forestales y de plagas de insectos; aprovechamiento de residuos agrícolas, evitando su quema en el terreno; posibilidad de utilización de tierras en barbecho con cultivos energéticos; independencia de las fluctuaciones de los precios de los combustibles provenientes del exterior, al no ser combustibles importados; mejora socioeconómica de las áreas rurales.

Además, la extracción de residuos forestales destinados a la producción de biomasa implica, a su vez, una serie de ventajas: favorece la regeneración natural de la masa principal; facilita la repoblación artificial de la masa forestal; posibilita el crecimiento de arbolado; incrementa la capacidad de aprovechamiento de productos forestales; facilita las operaciones selvícolas y los movimientos por el monte; mejora estéticamente el monte; y aumenta la capacidad de acogida recreativa.

Según datos del Plan de Energías Renovables (PER) del Ministerio de Industria, Turismo y Comercio (MITYC), en el año 2010, con un incremento de la potencia eléctrica con biomasa de 1695 MW y un incremento en la energía primaria procedente 
de biomasa térmica de 582,5 ktep, las emisiones evitadas de $\mathrm{CO}_{2}$ superarían los nueve millones de toneladas (7364191 t $\mathrm{CO}_{2}$ evitadas con biomasa eléctrica y 1788326 t $\mathrm{CO}_{2}$ evitadas con biomasa térmica). La heterogeneidad de recursos aprovechables es una característica intrínseca de los sistemas de producción de energía asociados a la biomasa. Ello aumenta su complejidad ya que cada proyecto necesita análisis específicos de disponibilidad, extracción, transporte y distribución.

En España, los principales desarrollos en el área de biomasa se han centrado en el uso de residuos industriales, tanto forestales como agrícolas. Respecto a la biomasa forestal, ha sido utilizada tradicionalmente en el sector doméstico mediante sistemas poco eficientes, algo que está cambiando debido a la llegada al mercado de sistemas de calefacción y agua caliente modernos, de alta eficiencia y comodidad para el usuario.

Los residuos y materiales susceptibles de ser utilizados en la producción de energía son: residuos forestales, residuos agrícolas leñosos, residuos agrícolas herbáceos, residuos de industrias forestales y agrícolas, cultivos energéticos. Dentro de los usos de la biomasa, las aplicaciones térmicas con producción de calor y agua caliente sanitaria son las más comunes. En un nivel menor de desarrollo se sitúa la producción de electricidad, pues precisa de centrales térmicas específicas, que conllevan inversiones elevadas y reducen el rendimiento. Esto explica el poco peso de la biomasa eléctrica en el cómputo global de esta energía, 837 ktep de consumo en 2010 frente a los 3915 ktep de las aplicaciones térmicas.

La cuantificación del carbono en la biomasa y la dinámica de fijación atendiendo al estado de conservación del monte es una información relevante y necesaria en la lucha contra el calentamiento global. Como se ha comentado anteriormente, son escasos los estudios referidos a la fijación de carbono por parte de especies arbustivas, dejando a un lado el estudio de su papel en la fijación del $\mathrm{CO}_{2}$ global. El territorio forestal en Castilla y León asciende a 4.8 millones de hectáreas, lo que supone el 51\% del territorio. En contraste, la superficie forestal europea es 10 puntos inferior (41\%) y la superficie forestal mundial es del $39 \%$. Por lo tanto, la superficie forestal per cápita es de 1.92 ha/hab., más de tres veces superior a la media española, 2.5 veces a la media mundial y casi 6 veces superior a la media europea (Junta de Castilla y León, 2014). La superficie ocupada por matorral en Castilla y León es significativa, superando las 900000 ha (19\% de la superficie forestal); los matorrales más abundantes son los brezales (Erica sp. y Calluna sp.) y los escobonales (Genista sp., Cytisus sp., etc) con 273000 ha y 149000 ha respectivamente, seguidos de los jarales (Cistus sp.) con una 
superficie cercana a 55000 ha (PEFC, 2009). Es por ello que se considera de gran importancia cuantificar la cantidad de carbono fijado por especies pertenecientes a dos de los géneros más abundantes de la Comunidad: Erica arborea L. y Cistus ladanifer L.

Además, no hay que olvidar las características fisiológicas y fenológicas propias de estas especies. Por ejemplo, la especie C. ladanifer produce una gran cantidad de hojarasca de lenta degradación formadora y creadora de suelo. Esta especie se desarrolla en suelos pobres en nutrientes, siendo capaz de fijar carbono y desarrollarse rápidamente a pesar de las limitaciones nutricionales e hídricas (Núñez, 1989). 


\subsection{CARACTERIZACIÓN DE JARALES Y BREZALES. SISTEMÁTICA, FILOGEOGRAFÍA Y USOS DE LAS ESPECIES ARBUSTIVAS BAJO ESTUDIO}

\subsubsection{Características generales de los jarales. Morfología, distribución y usos de Cistus ladanifer}

\subsubsection{Características generales de los jarales}

Etimológicamente, la palabra castellana jara proviene del árabe andalusí šá 'ra, y ésta del árabe clásico $̌ s a ' r a \bar{\prime}$ ', que significa tierra llena de vegetación. Su clasificación botánica indica que pertenece al género Cistus, de la familia Cistaceae y del oden Violales; de clase Magnoliopsida y división Magnoliophyta del reino Plantae.

Los jarales están constituidos por especies del género Cistus, las cuales forman comunidades arbustivas de carácter serial de bajo nivel evolutivo asociadas a la fase regresiva de encinares y bosques mixtos de encina y alcornoque. Cuando los jarales son puros están formados únicamente por $C$. ladanifer, aunque generalmente aparece acompañada de otros arbustos o matorrales y se distribuyen en manchas de gran extensión. Son representativos de los sistemas agro-silvo-pastorales mediterráneos, pues debido a su carácter pionero aparecen en las primeras etapas de sucesión (colonizando suelos degradados, principalmente tras el abandono de tierras agrícolas o incendios reiterados) y forman grandes extensiones con espesas coberturas. De hecho, el siguiente fragmento ha sido extraído de las Anotaciones al libro de la montería del Rey Alfonso XI, por José A. Valverde:

Los jarales (Cistus ladanifer principalmente) se extienden con los fuegos y colonizan suelos pobrísimos. Tras los incendios los pies de jara y madroño se transforman en peligrosísimas lanzas que impiden el paso de las caballerías (de los tractores ahora) y admiten solo el hombre cazador a pie. Con el tiempo, estos bosques que no ceden al fuego e impiden el paso de caballos han venido a constituir el refugio predilecto de la gran fauna (ciervos, jabalies, osos), que tiene en ellos sus querencias o camas.

Además, al ser una especie pirófila activa, es capaz de mantener sus semillas sin germinar durante años, amoldarse a suelos pobres y otras situaciones de escasez; esta adaptación a distintos factores como temperatura, precipitación, $\mathrm{pH}$... hace que su nicho 
ecológico sea muy extenso, al adaptarse a hábitats muy variados con condiciones ambientales diferentes, como los extremos termopluviométricos del clima peninsular.

\subsubsection{Morfología, distribución y usos de Cistus ladanifer}

Conocida como jara pringosa, $C$. ladanifer es un arbusto siempre verde que en condiciones óptimas puede llegar a medir hasta 2,5 o incluso hasta $4 \mathrm{~m}$, pero que habitualmente no supera los $2 \mathrm{~m}$. Las hojas son opuestas, carecen de estípulas y se sueldan entre sí en la base; son enteras, alargadas y estrechas, alcanzando hasta $10 \mathrm{~cm}$ de largo y 1,5 cm de anchura. En etapas iniciales están fuertemente impregnadas de una sustancia pegajosa -el ládano- que les da un aspecto lustroso y se adhiere fácilmente a manos y ropa. Florece de abril a junio, con flores muy grandes, de hasta $10 \mathrm{~cm}$ de diámetro, con 3 sépalos y 5 pétalos de un color blanco puro que en ocasiones presentan una mancha purpúrea en la base, denominándose jara de las cinco llagas. El fruto es una cápsula globosa con 7-10 compartimentos y que se abre en la madurez en otras tantas valvas (Figura 1).
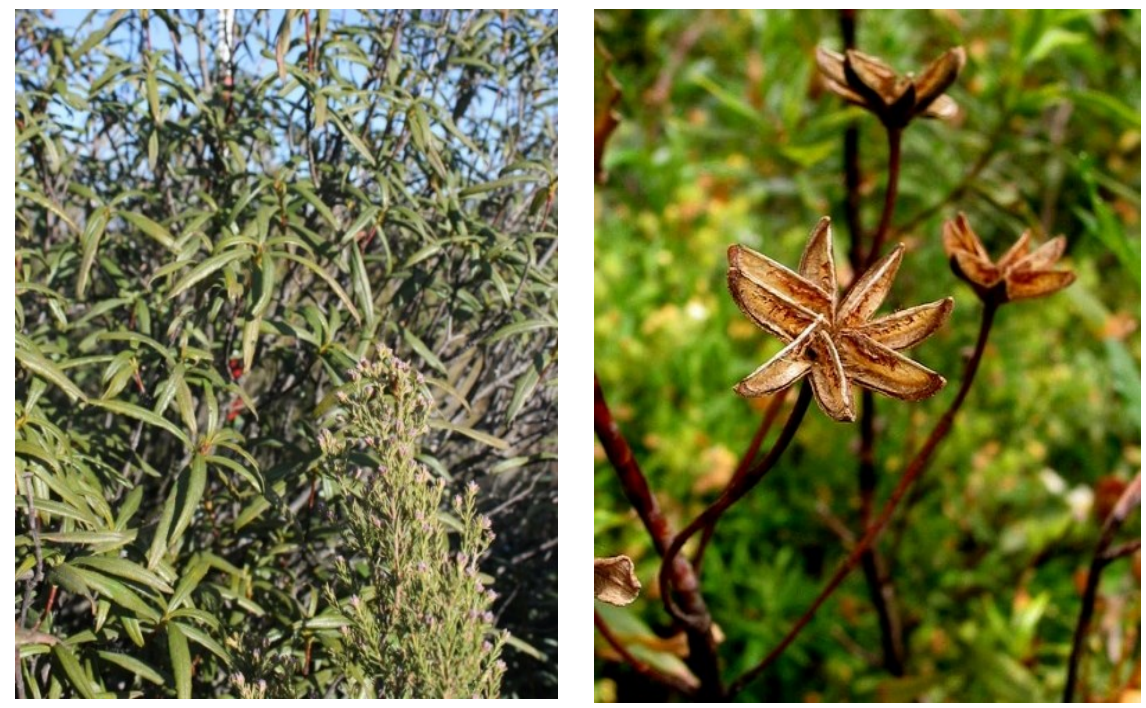

Figura 1. Ejemplar de C. ladanifer con una rama de E. arborea en primer plano y un enfoque selectivo de cápsulas de jara. (Tomado de M. Casares, 2017)

C. ladanifer es una especie autóctona de la Península Ibérica (Figura 2), que habita en la región mediterránea occidental y en las Islas Canarias. Es muy frecuente en toda la parte silícea y de clima mediterráneo de la Península, tanto en España como en Portugal, faltando sólo en el norte. 


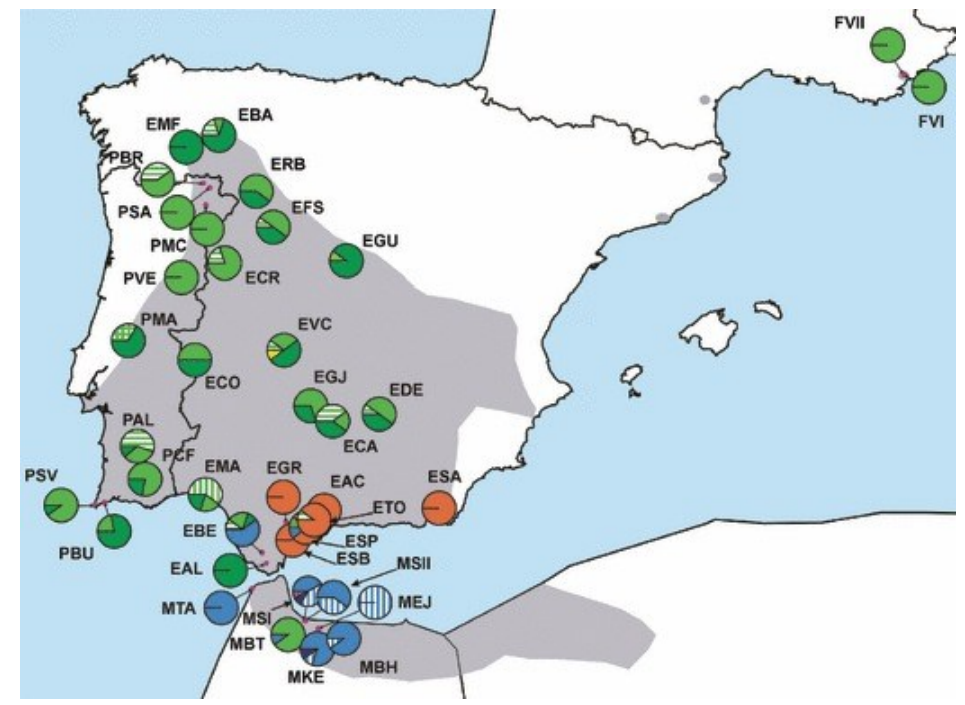

Figura 2. Mapa de distribución de Cistus ladanifer en la Península Ibérica (haplotipos). Tomado de Quintela-Sabarís et al., 2010

C. ladanifer crece en matorrales de sitios secos y soleados, generalmente en ambiente de encinar, en terrenos silíceos (no calcáreos, tales como granitos, gneises, cuarcitas y pizarras), especialmente en los suelos más degradados o menos evolucionados, por lo que su presencia suele indicar suelos muy pobres. Se extiende desde el nivel del mar, donde puede vivir incluso en las arenas de las dunas costeras hasta unos $1500 \mathrm{~m}$ de altitud. Su área coincide en líneas generales con la de la encina, ocupando el espacio que deja ésta cuando sus bosques se queman o talan y el suelo carece de cal, aunque en ocasiones se cultiva como ornamental.

En Castilla y León, se ubican fundamentalmente en áreas potenciales de los encinares silicícolas; principalmente en Zamora y León (Figura 3), sobre todo en las franjas más occidentales (El Bierzo, entorno de la Maragatería, zonas más bajas y secas de los páramos leoneses, Aliste, Sayago, Alba y en menor medida La Carballeda), son muy frecuentes en Salamanca, en el ámbito de las dehesas y montes de encina, aunque también se hallan jarales al sur de Ávila y puntualmente en Valladolid.

En nuestra región, los jarales son habitualmente monoespecíficos, aunque en ocasiones aparecen en agrupaciones mixtas con brezales (sobre todo en León y norte de Zamora) o incluso escobonares y retamares. Se asientan sobre laderas graníticas y sobre esquistos pizarrosos. Se ven favorecidos por los incendios, a los que sobreviven gracias a la elevada proporción de semilla cuya germinación se ve favorecida por el fuego (plantas pirófilas). Extensos jarales pueden ser considerados una fase poco madura en el desarrollo de manchas de vegetación, en las que suelen entrar a formar parte y pasan a dominar cuando son degradadas o incipientes. 


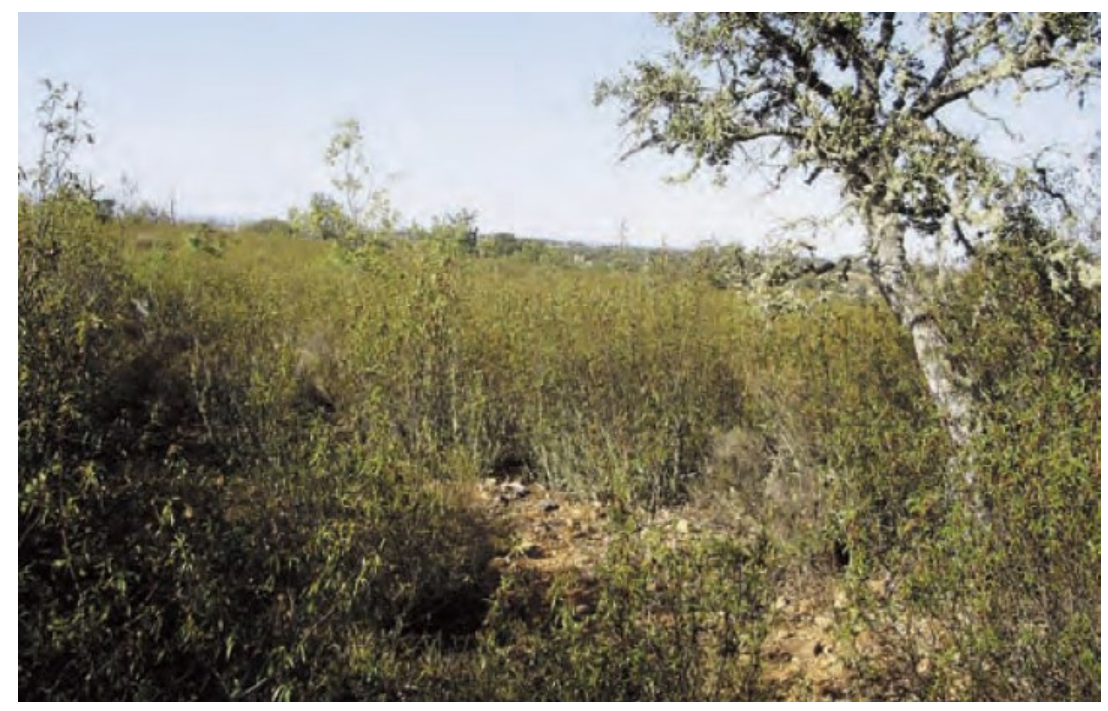

Figura 3. Jaral de Cistus ladanifer en tierras zamoranas

El nombre específico de la jara pringosa, ladanifer, alude a la producción por la misma de ládano y que supone un mecanismo de defensa frente a la sequía, de manera que refleja la radiación UV solar y preserva así el tejido vegetal y evita su desecación. Además, su abundancia le permite competir con éxito frente a distintas especies, ya que puede inhibir el crecimiento de otras plantas, lo que explica también su carácter pionero. No obstante, tiene la capacidad de asociarse mediante micorrizado a especies fúngicas con alto valor de mercado como Boletus edulis Bull. Esta resina se empleó antiguamente como medicinal: se le atribuían propiedades sedantes, y entraba en la composición del emplasto regio utilizado para la curación de las hernias. El método de recolección implicaba un rebaño de cabras que se hacía circular por un jaral denso, peinándose luego la barba y el pelambre del animal para obtener el producto, obteniéndose un ládano aromatizado a cabruno. También se han empleado hombres con mandiles de cuero o se ha extraído por inmersión de la planta en agua hirviendo. Actualmente se usa más en perfumería como fijador de perfumes pero ha vuelto a recuperar cierto protagonismo medicinal como revulsivo y antiespasmódico, para tratar las gastritis y úlceras gastroduodenales. En uso externo se usa para combatir el reumatismo y las neuralgias. Pero el uso de la resina no es muy recomendable por ser bastante tóxica.

En la actualidad se está estudiando el potencial antifúngico de los extractos de Cistus ladanifer. Un estudio (Barrio, Dueñas et al., 2013) realizado frente a especies de Candida (Candida albicans, C. glabrata and C. parapsilosis ha mostrado que Cistus ladanifer inhibe su crecimiento de un modo eficaz ( $\mathrm{MIC}<0.05 \mathrm{mg} / \mathrm{mL}$ ), especialmente 
frente a C. glabrata, donde el extracto estudiado fue capaz de reducir las concentraciones por debajo de $0.05 \mathrm{mg} / \mathrm{mL}$ en al menos tres registros y la inhibición total del crecimiento en concentraciones superiores a $0.625 \mathrm{mg} / \mathrm{mL}$. Esta acción antifúngica ha de ser referida al contenido en ácidos fenólicos y derivados $(4 \mathrm{mg} / \mathrm{g} \mathrm{de}$ extracto), derivados de ácido elágico (30 mg/g de extracto), y flavonoides tales como catequinas, flavonoles y flavonas (4 mg/g de extracto). Los derivados de ácido elágico fueron el grupo más abundante, siendo el punicalagin gallate -un derivado de punicalaginas atacado con ácido gálico-, quien aparecía en mayor cantidad (16 mg/g de extracto).

\subsubsection{Características generales de los brezales. Morfología, distribución y usos de Erica arborea}

\subsubsection{Características generales}

Etimológicamente, la palabra brezo procede del latín hispánico broccius, y éste del celta vroicos; cónfer galés grug, irlandés antiguo froech y gaélico fraoch. Su clasificación botánica indica que pertenece al género Erica, de la familia Ericaceae y del oden Ericales; de clase Magnoliopsida de la división Magnoliophyta del reino Plantae.

También denominada urce o urce branca en gallego y urze-branca en portugués, siendo urce la primera referencia que existe sobre esta especie, debida a Teofrasto (filósofo griego y contemporáneo de Aristóteles que escribió dos tratados de botánica, Historia de las plantas, donde describe más de 450 plantas y expone algunas nociones de fisiología vegetal, y Sobre las causas de las plantas, donde unifica y amplía el conocimiento sobre plagas y enfermedades). Es Dioscórides (médico, farmacólogo y

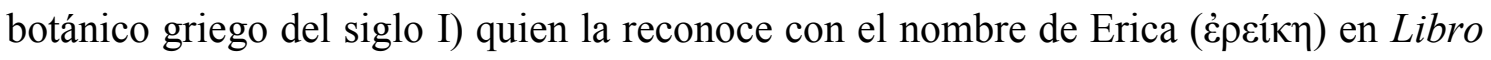
$I(88)$.

Los brezales son matorrales de bajo nivel evolutivo, propio de suelos desarrollados sobre sustratos ácidos o básicos acidificados y dominado por diversas ericáceas entre las que es común Erica arborea. Se trata de comunidades de estructura simple, con dominio de un estrato arbustivo bajo, aunque en ocasiones son frecuentes (y abundantes) otras leñosas de porte alto, como enebros y espinos. La cobertura de los arbustos dominantes es variable, aunque en la mayor parte de los casos se trata de matorrales densos. El recubrimiento de las herbáceas puede ser importante (hasta 50\%$70 \%$ ), dejando un porcentaje de suelo desnudo no muy elevado $(<15 \%)$. 
El ramón de las ericáceas presenta escasa palatabilidad y valor nutritivo, debido a sus bajos porcentajes en proteína y digestibilidad de la materia orgánica, y a sus altos contenidos en fibra, por lo que resultan poco apetentes para el ganado. No obstante, pueden constituir un alimento de volumen que es consumido cuando otros recursos son escasos o están agostados. En este sentido, la utilización de los brezos es muy baja siempre que la disponibilidad de especies herbáceas apetecibles se sitúe por encima de una altura mínima $(2-3 \mathrm{~cm})$. A medida que la disponibilidad se reduce por debajo de dicho nivel, el porcentaje de brezo en la dieta se incrementa en el ovino y de forma menos notoria en el vacuno (Osoro et al., 1999). El ovino realiza un pastoreo selectivo de los brotes nuevos y tiernos de las ericáceas, mientras el vacuno y equino no las seleccionan de manera activa en condiciones normales de pastoreo, aunque las pueden llegar a consumir; su efecto sobre estas comunidades se debe más al pisoteo. En general, se pastan en primavera, verano y otoño por ganado mayor y menor. En el área submediterránea pueden formar parte de zonas de pastoreo invernal que, tradicionalmente, son empleadas por ganado mayor. El brezo, además, es muy apreciado en apicultura y se utilizan con asiduidad en explotaciones apícolas.

\subsubsection{Morfología, distribución y usos de Erica arborea}

Erica deriva del griego erikein-ereiké, que significa romper, ya que una vez secas, sus ramas se tronchan con facilidad y sus hojas se desprenden fácilmente. Su nombre latino aludía a todas las especies de brezo conocidas, incluso a la brecina (Calluna vulgaris (L.) Hull). El epíteto específico arborea alude al porte que puede alcanzar esta planta.

Conocido como brezo blanco o brezo castellano, se trata generalmente de un arbusto elevado y muy ramoso, de 2 a $4 \mathrm{~m}$, que en la Península Ibérica alcanza el porte arbóreo en algunos puntos del sur peninsular, como el Parque Natural de los Alcornocales (Cádiz). Entre otras razones, esto es debido a que sus cepas son muy apreciadas y se han cortado para diversos usos a lo largo del tiempo. No obstante, llega hasta los $10 \mathrm{~m}$ en Canarias y alcanza los 15-20 m en el este de África, en las faldas del Kilimanjaro (Figura 4). 


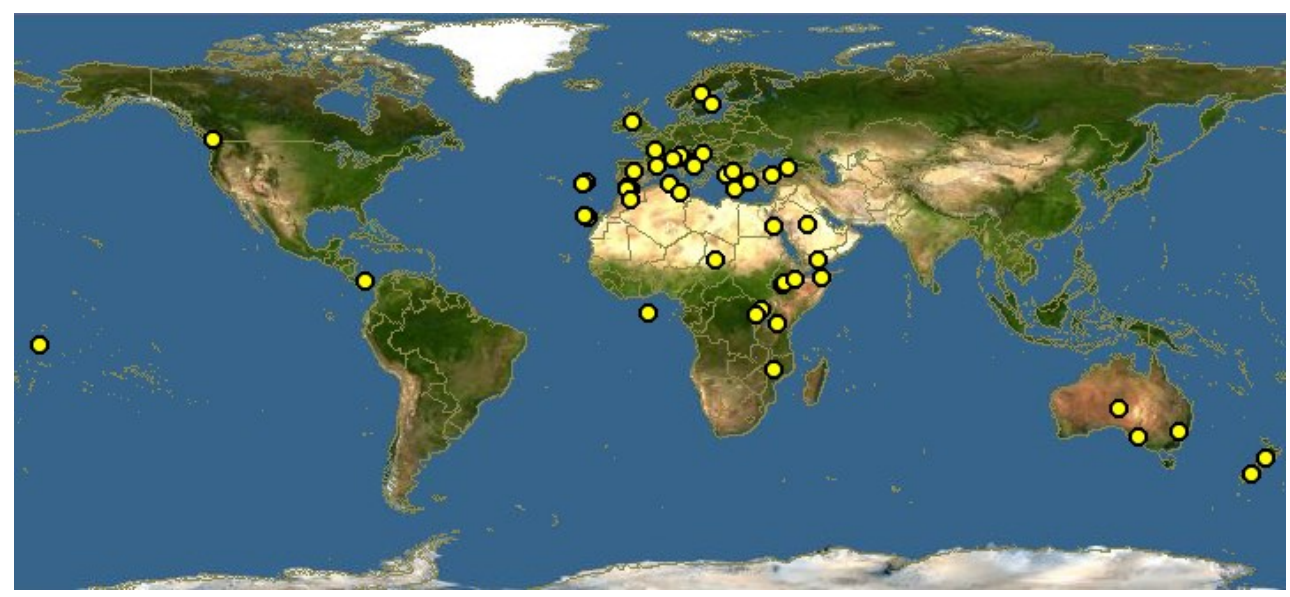

Figura 4. Mapa de distribución mundial de Erica arborea (imagen tomada de Discover Life http://www.discoverlife.org/mp/20q?search=Erica+arborea)

Las ramillas jóvenes son blanquecinas y tienen tricomas desiguales. Su corteza es parda y fibrosa, y al madurar se desprende en tiras alargadas y finas. De hojas lampiñas, persistentes, simples, finas y muy estrechas, en forma de acícula corta con los bordes revueltos hacia el envés, de manera que parece que tengan un surco en la cara inferior. Miden 3-8 $\mathrm{mm}$ de largo por 0,5-0,7 $\mathrm{mm}$ de ancho, y se disponen en las ramillas en verticilos de 3 o 4 como las aspas de un ventilador. Las flores son blanquecinas, a veces sonrosadas, y muy pequeñas, de hasta $3 \mathrm{~mm}$ de longitud, con el pedúnculo que las sostiene bastante más largo, y crecen en grupos terminales de forma piramidal. Tienen forma de campanita cerrada y estrecha, y cuando maduran los estambres permanecen en el interior, a diferencia de otras especies de brezos cuyos estambres sobresalen asomando las anteras — parte superior del estambre que contiene el polen - al exterior (Figura 5).
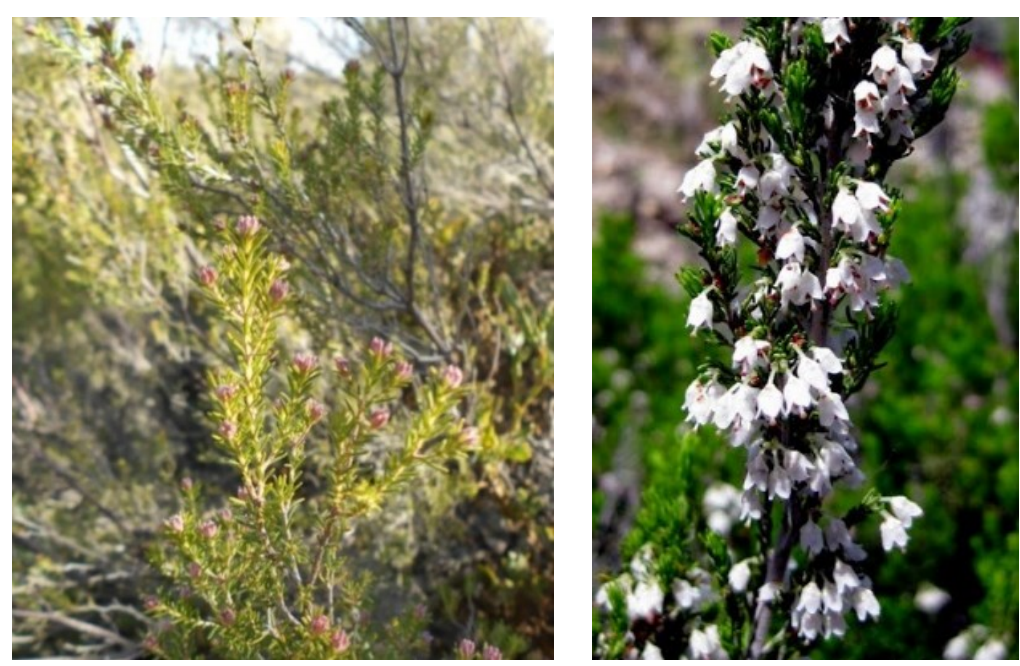

Figura 5. Ejemplar de E. arborea y un enfoque selectivo de un ramillete en flor (tomado éste de J.L. Sáez, 2017) 
El brezo blanco es una especie de área muy dispersa. Habita en la región mediterránea, Canarias, Madeira, norte y este de África, Asia Menor, Cáucaso. En la Península y Baleares es común y se extiende de forma desigual. Es una planta típica de sustratos ácidos y característica de bosques húmedos o asociada a la humedad que proporcionan los cursos de agua. Se desarrolla en el seno de hayedos, pinares y robledales, y se agrupa en extensas formaciones. Crece desde el nivel del mar hasta los $2000 \mathrm{~m}$ de altitud. En Canarias y África alcanza portes arbóreos que llegan a superar los $10 \mathrm{~m}$. Sorprende ver bosques densos de esta especie, que en la Península y Baleares no suelen pasar de un arbusto ramoso.

En Castilla y León, los brezales de Erica arborea ocupan las laderas más frescas y húmedas, con suelos que retienen suficiente cantidad de agua (Figura 6). Suelen llevar plántulas de especies arbóreas principales, como robles o abedules o pioneras secundarias, como serbales. El brezal blanco puede aparecer sobre formadores de turba, en ocasiones de notable espesor.

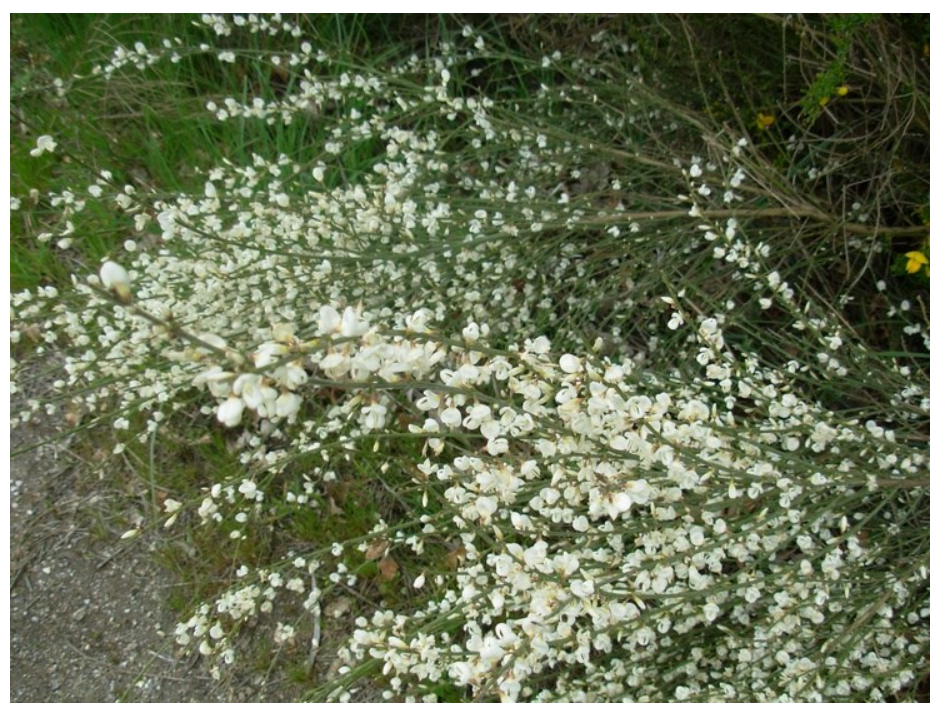

Figura 6. Brezal de Erica arborea en tierras zamoranas

Entre los usos tradicionales del brezo blanco destaca -al igual que con otras especies de brezos y afines- la fabricación de escobas, debido a su denso y fino ramaje. También se utiliza para confeccionar tejadillos, empalizadas y cubiertas que pongan al resguardo del viento, el sol, la lluvia y las miradas ajenas. Antiguamente, se denominaba brezo a la cama armada sobre zarzos. Su leña es un excelente combustible y una de las mejores para fabricar carbón vegetal, apreciado en hornos y fraguas. Su 
madera es roja como el carmín, dura y densa, muy buscada por ebanistas y torneros para pequeñas tallas.

Las raíces son muy valoradas para fabricar pipas de fumar (ver cita), como sucede en Cataluña y el norte de África, donde las extraen para luego exportarlas, principalmente a Inglaterra. En el Parque Natural de los Alcornocales, en Cádiz, la raíz de este brezo es muy buscada, debido a que sus cepas tienen un bello veteado que al pulir proporciona figuras y arabescos muy valorados. Con ella se confeccionan los salpicaderos y pomos de las palancas de cambio de los coches de lujo de la casa Ferrari.

En "El signo de los Cuatro" de Sir Arthur Conan Doyle leemos:

«Mi actividad se ha extendido últimamente al Continente — dijo Holmes, al poco rato, mientras llenaba su vieja pipa de raíz de brezo-».

Otro uso del brezo es como planta ornamental, si bien hay otras especies de brezo de flores muy vistosas. No obstante, la medicina popular utilizaba la copa y la flor del brezo blanco para aplicarlas como emplasto con el fin de curar las mordeduras de serpientes. En los últimos tiempos también se ha utilizado en repoblaciones forestales con planta autóctona. Asimismo es conocida la denominada "tierra de brezo", un sustrato muy nutritivo para el cultivo de plantas de jardinería que se va formando durante años bajo el suelo de los brezales umbrosos.

Es una planta melífera, cuyas flores producen abundante néctar y a la vez su polen es utilizado por las abejas (Terrab, 2004). Conocida desde la antigüedad, el brezo estaba consagrado a dos diosas, a Venus Ericina, quien personificaba el amor impuro y era la diosa patrona de las cortesanas, y a la diosa conocida como Uroica ( $U r$, nombre del brezo entre los celtas), deidad que regía el solsticio estival con su cohorte de diligentes abejas (Mc Innes, 2014). La miel de brezo es un constante en la industria de la apicultura, apreciada internacionalmente por su sabor fuerte y penetrante, con un gusto ácido, aromático y ligeramente amargo.

Según el antropólogo y escritor Juan Luis Arsuaga: «Los diferentes clanes escoceses se distinguían entre sí por el color de la planta que llevaban en el gorro (y no, como suele pensarse, por los colores del estampado o “tartán”); por ejemplo, el brezo rojo y el brezo blanco podían corresponder a clanes diferentes». En este sentido, la novela Brezo blanco, de la escritora Nieves Hidalgo, trata de los conflictos entre los clanes escoceses.

El brezo siempre ha cubierto vastas extensiones de terreno en Escocia y sus flores han sido utilizadas para fabricar cerveza desde el año 2000 a.C. 


\section{3. ÁMBITO DE ESTUDIO}

Las formaciones vegetales precisan de ciertas sustancias fundamentales, además de la energía procedente del sol, para su existencia: el agua, que llega al suelo mediante las precipitaciones, y los nutrientes, que dependen de la naturaleza de la roca madre, el grado de evolución edáfica y el aporte de materia orgánica, y cuyo aprovechamiento -a su vez- está ligado al del agua, puesto que las plantas toman los nutrientes disueltos en ella.

\subsubsection{Situación administrativa y económica}

El estudio se ha desarrollado en una zona próxima a la población de Congosta, en el municipio de Ayoó de Vidriales de la provincia de Zamora (Castilla y León, España). El término municipal del mismo nombre comprende las localidades de Ayoó de Vidriales, Carracedo de Vidriales y Congosta (Figura 7); pertenece a la comarca de Benavente y Los Valles (agrupación voluntaria de municipios limítrofes con características geográficas, económicas, sociales e históricas afines) y constituye la mancomunidad de servicios Valle del Tera, cuyo objetivo es la gestión de determinados servicios públicos municipales (LOREACYL 14/2007, de 30 de noviembre). La zona está enclavada en el límite Sur de la Sierra Cabrera, en la transición de ésta a la Sierra de Carpuria, siendo ésta última la que forma el Valle de Vidriales, por lo que el relieve se caracteriza por una continuación de pequeñas ondulaciones, más o menos acentuadas, en las cuales se encajan pequeños valles, marcados por intensos procesos erosivos, todos ellos con dirección predominante hacia el Sureste.
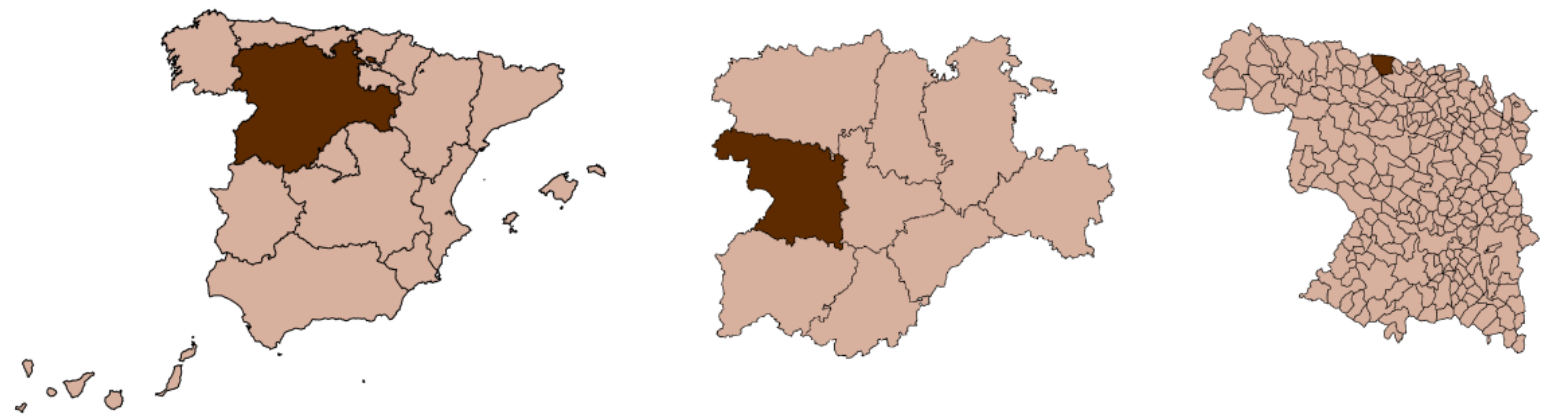

Figura 7. Localización de la zona de estudio: Congosta, Ayoó de Vidriales, Zamora, España

Con una superficie de $\sim 60 \mathrm{~km}^{2}$ cuenta con una población total de 349 habitantes en el año 2016 (INE), la actividad económica principal es la Agricultura, ganadería, 
caza y selvicultura; con 5399 ha de explotaciones (siendo 5194 en propiedad), 658 ha son tierras labradas, 1380 ha destinadas a pastos permanentes, 1273 ha de especies arbóreas forestales y 2089 de otras tierras no forestales. De las 131 explotaciones agrícolas, 118 tienen menos de 5 ha, 568 ha están destinadas a herbáceas y 89 a viñedo. Las unidades ganaderas del municipio ascienden a 88 bovinos, 234 ovinos, 24 caprinos, 178 porcinos, 24 equinos y 13 aves. Además, son cinco los cotos de caza del municipio, privados, y que abarcan un total de 6191.7 ha. Ayoó también cuenta con 13.50 ha de superficie de la Red Natura 2000, bajo la denominación de Lugar de Interés Comunitario (LIC) Lagunas de Tera y Vidriales (VV.AA., 2013).

\subsubsection{Características físicas del entorno}

La superficie de matorral mixto analizada se encuentra entre las 25 ha correspondientes a la zona $\mathrm{B}$ del proyecto LIFE+ Operation $\mathrm{CO}_{2}$ : Integrated agroforestry practices and natura conservation against climate change cuyo objetivo principal es demostrar la viabilidad de los proyectos forestales y agroforestales en el secuestro de carbono en Europa; enmarcado en las siguientes condiciones ambientales:

En términos de clasificación, el clima de la zona de Ayoó de Vidriales puede enclavarse dentro del tipo Mediterráneo Templado, que por otro lado es el tipo climático predominante en la totalidad de la provincia. El régimen de precipitaciones, sin embargo, es algo más húmedo de lo normal para este tipo climático que sería de unos $440 \mathrm{~mm} / \mathrm{año}$, alcanzándose en la zona precipitaciones de unos $850 \mathrm{~mm} / \mathrm{año}$. La caracterización agroclimática de la zona la asigna un tipo de verano "maíz", M; un tipo de invierno "avena fresco", av; y un régimen de humedad es "Mediterráneo seco", Me. Esta caracterización agroclimática tomada del Mapa de Cultivos y Aprovechamiento de la Provincia de Zamora (MAPA, 1984) hay que considerarla con la puntualización de la altura mayor de Ayoó de Vidriales con respecto a la comarca en la que se enclava, es decir de Benavente y los Valles, de clima más benigno. El índice de potencialidad agroclimática de L. Turc para el secano es de 12, siendo para el regadío de 32 . En cuanto a las heladas, las temperaturas por debajo de $\operatorname{los} 0^{\circ} \mathrm{C}$, son muy habituales, comenzando generalmente en octubre y finalizando sobre el mes de mayo.

Geológicamente, pertenece al Mioceno (era del Cenozoico, periodo Terciario y subsistema Neógeno), piso Pontiense-Vallesiense-Vindoboniense superior, correspondiente con una litología de arcillas, arenas y conglomerados (Hoja 269- 
Arrabalde, del Mapa Geológico Nacional (MAGNA) del Instituto Geológico y Minero de España, IGME).

El suelo está dentro del Orden de los Inceptisoles, caracterizados por ser suelos jóvenes, en evolución, poco meteorizados y que comienzan a mostrar el desarrollo de los horizontes de los que se componen. El suborden de estos suelos es el Ochrept, pues tiene un epipedon úmbrico, mientras que el subgrupo es el Xerochept debido a su régimen de humedad xérico. (Mapa de suelos para España del Sistema Español De Información de Suelos (SEISnet), basado en las claves de Taxonomía de Suelos del United States Department of Agriculture, USDA).

\subsubsection{Flora}

La importancia de la vegetación radica en el papel que desempeña este elemento como productor primario de casi todos los ecosistemas, así como en sus importantes relaciones con el resto de los componentes bióticos y abióticos del medio, tanto en sí misma como por ser un componente relevante de los ecosistemas y del paisaje. Además, mejora la estructura y porosidad del suelo, favoreciendo la infiltración y reduciendo la escorrentía y la evaporación.

\section{Vegetación potencial}

Las series de vegetación indican todo el conjunto de comunidades vegetales o estadios que pueden hallarse en unos espacios teselares afines como resultado del proceso de la sucesión, lo que incluye tanto las cualidades mesológicas, geográficas y florísticas de la asociación representativa de la etapa climax o cabeza de serie, como las de las asociaciones iniciales o subseriales que pueden reemplazarla. Siendo la vegetación potencial la comunidad vegetal estable que existiría en un área dada como consecuencia de la sucesión progresiva, sobre todo si el hombre dejase de alterar los ecosistemas naturales terrestres (Rivas-Martínez, 2004).

Cada serie está caracterizada por el piso bioclimático, la vegetación azonal y la región, siendo: la zonificación en función del gradiente término altitudinal, la vegetación que se desarrolla cuando las condiciones del suelo (salinidad, yesos, etc.) predominan sobre el resto de las condiciones ambientales del lugar, y un territorio extenso con especies, géneros e incluso familias propias, respectivamente. La zona de estudio se encuentra en el piso supramediterráneo, al que se le atribuye una temperatura 
media anual de $13^{\circ} \mathrm{C} \mathrm{a} 8^{\circ} \mathrm{C}$, la temperatura media de las mínimas del mes más frío de $1^{\circ} \mathrm{C}$ a $-4^{\circ} \mathrm{C}$, la temperatura media de las máximas del mes más frío de $9^{\circ} \mathrm{C}$ a $2^{\circ} \mathrm{C}$, el índice de termicidad $(\mathrm{T}+\mathrm{m}+\mathrm{M})$ de 210 a 60 y heladas entre los meses de septiembre a junio. Presenta a su vez una vegetación azonal de series climatófilas, dentro de la región mediterránea. Con estas características, pueden darse tres tipos de series de vegetación diferentes (Rivas-Martínez, 1987):

- Serie supramesomediterránea salmantina y orensano-sanabriense subhúmeda silicícola de Quercus pyrenaica (roble melojo). Genisto jalcatae-Querceto pyrenaicae sigmetum (serie 18e).

- Serie supramesomediterránea salmantina lusitano-duriense y orensano-sanabriense silicícola de Quercus rotundijolia (encina). Genisto hystricis-Querceto rotundijoliae sigmetum (serie 24b).

- Serie supramesomediterránea carpetana occidental, orensano-sanabriense y leonesa húmedo-hiperhúmeda silicícola de Quercus pyrenaica (roble melojo). Holco mollisQuerceto pyrenaicae sigmetum (serie 18b).

\section{Vegetación actual}

El área elegida tiene una vegetación mixta de matorral, cuyas especies predominantes son Erica arborea L. (Ea) y Cistus ladanifer L. (Cl), propuestas por Rivas Martínez como vegetación potencial en las series de vegetación de la zona: $E$. arborea, Db, 5g, 5h, 8a, 8d, 9a, 9b, 1lb, 16a, 16b, 16e, 17, 18e, 18d, 18h, 21a, 21e, 23a, 23b, 23e, 25, 35 y C. ladanifer, 24a, 24b, 24e, 24d.

Entre la vegetación arbórea encontramos Pinus pinaster Aiton (variedad mediterránea, xerófila, ocupa terrenos desde el nivel del mar hasta los $1900 \mathrm{~m}$ de altitud, en terrenos silíceos y calizas); Quercus pyrenaica Willd (especie ibérica por excelencia, exclusiva de terrenos silíceos de montaña en la mitad Oeste de la Península, medra en laderas y páramos desde los 800 a los 1700 m y resiste la sequía estival, el frío intenso y las heladas tardías. Valor protector muy elevado por desarrollarse a menudo en terrenos pobres. De interés paisajístico por su crecimiento rápido y vital, sobre todo en monte bajo y una vez que los brinzales enraízan bien; Quercus ilex subsp. ballota (Desf.) Samp. — muy extendida en terrenos continentales por la Península Ibérica(Figura 8). En estas formaciones de encinares cabe destacar que los únicos que aparecen 
en la zona estudidada, de forma pura y con un desarrollo óptimo, son los que se encuentran en el paraje denominado "Monte Cocho".
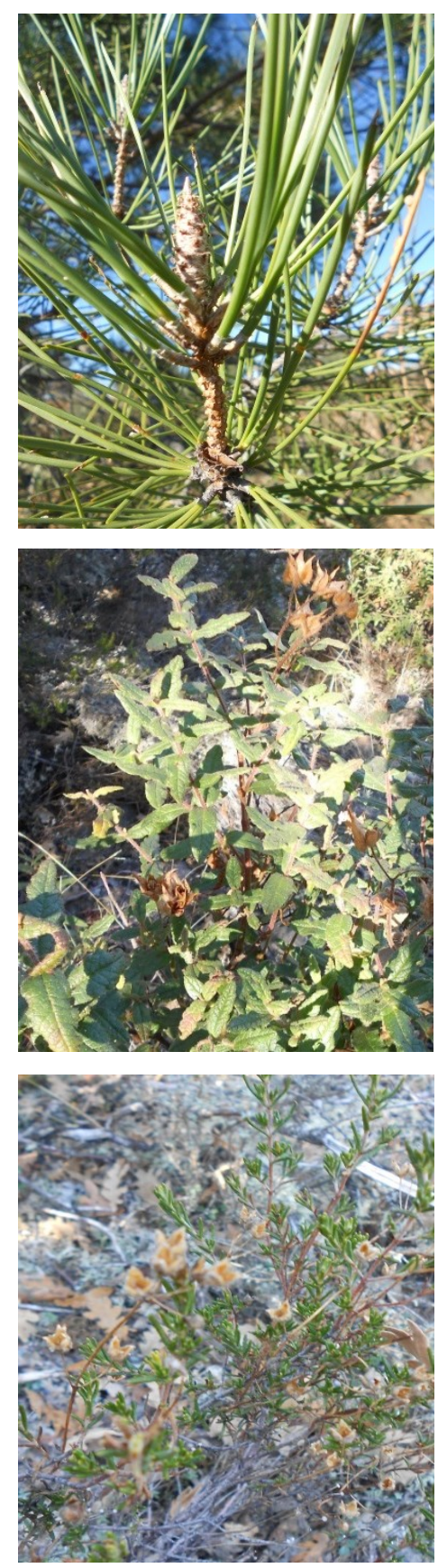
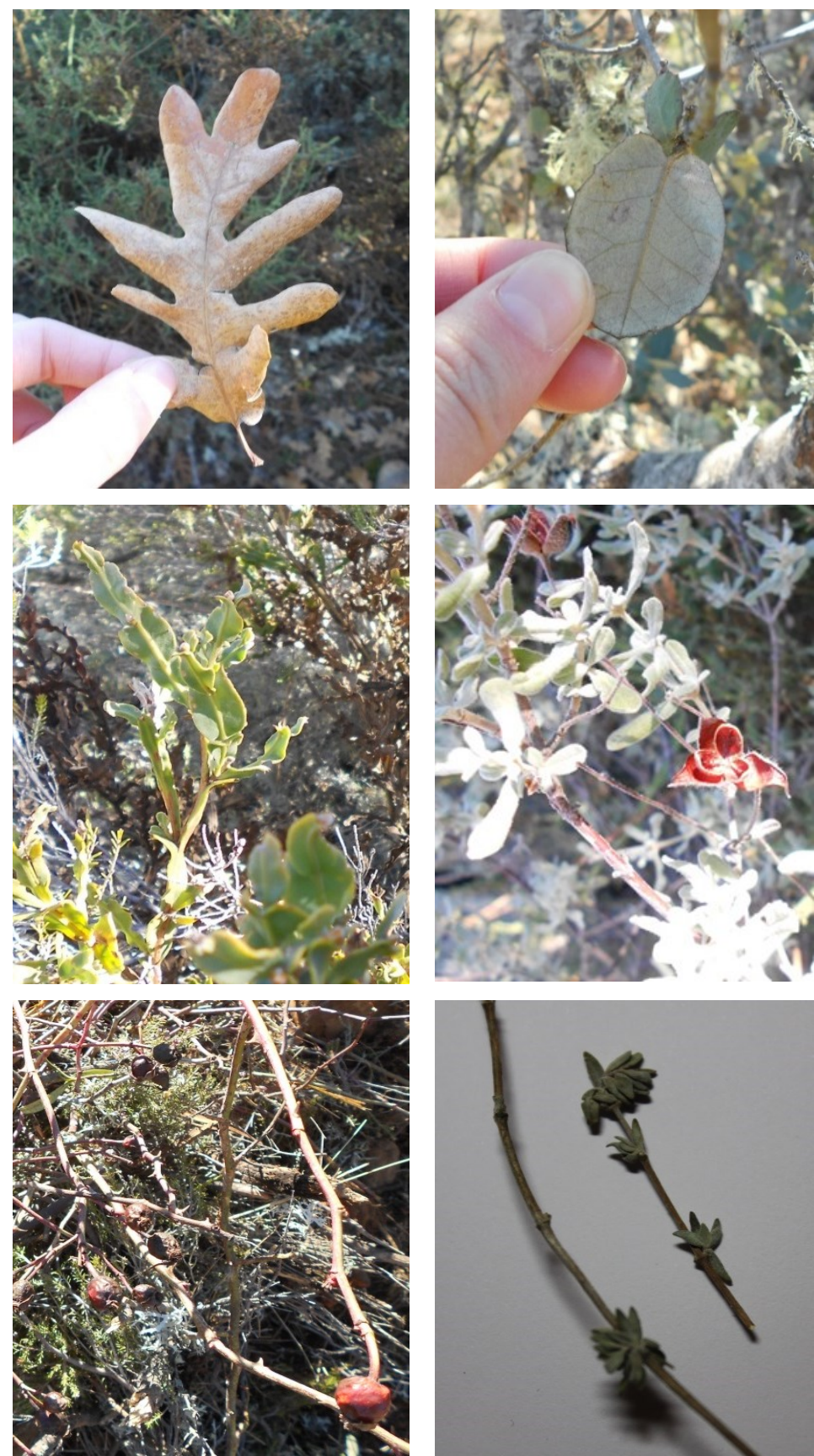
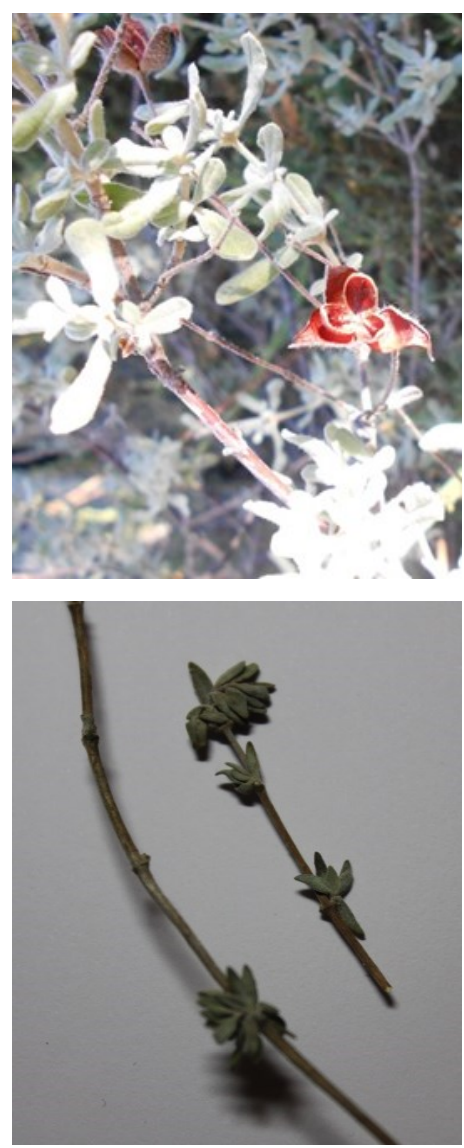

Figura 8. De izquierda a derecha y de arriba abajo: Yema y ramilla de Pinus pinaster, hoja de Quercus pyrenaica, envés foliar de Q. ilex subsp. ballota, cápsulas y planta de Cistus psilosepalus, Halimium atriplicifolium, Halimium sp., Genista tridentata, Rosa sp. y muestra de Thymus sp. 
Entre la vegetación arbustiva y de matorral, además de las dos especies bajo estudio, encontramos: brezo común, Calluna vulgaris; brezo bermejo, Erica cinerea; brezo de escobas, Erica scoparia; carpazo, Cistus psilosepalus Sweet (en suelos frescos, vaguadas y torrenteras; frecuentemente asociado a Erica lusitanica); jara blanca Halimium atriplicifolium (Lam.) Spach (también llamada jara del diablo, sobre terrenos silíceos, aunque tolera también los calizos); carquesa o carqueja, Genista tridentata L., (=Chamaespartium tridentatum), usual en terrenos silíceos y en bosques aclarados de pinos o alcornoques; Rosa sp., frecuente en setos y espinares, orlas forestales y bosques; generalmente en suelos frescos y ambientes con cierta humedad; desde el piso colino al subalpino, pisos meso- y supramediterráneo; tomillo común, Thymus sp.; y Satureja intricata Lange, que aparece en tomillares y matorrales pulvinulares de los páramos y montañas interiores de clima mediterráneo duro y continental (Figura 8). 


\subsection{MARCO DE REFERENCIA. ESTADO DEL CONOCIMIENTO SOBRE LAS CARACTERÍSTICAS Y APROVECHAMIENTO DE LAS ESPECIES ARBUSTIVAS OBJETO DE ESTUDIO}

\subsubsection{Sumideros de carbono}

La capacidad de fijación de $\mathrm{CO}_{2}$ por el matorral no es un área de estudio rica en antecedentes. La mayor parte de los existentes proceden de publicaciones de los Departamentos de Ingeniería Agrícola y Forestal (Hernández, Martín y Ruíz, 2007) y Ciencias Agroforestales (Moyano y Charro, 2007) de la Universidad de Valladolid; del Grupo de Investigación de Estudios Funcionales de Ecosistemas Mediterráneos de la Universidad de Extremadura (Alías-Gallego, 2009; García-Rosa et al., 2014), del grupo de investigación formado por Montero, Alonso, Ruíz-Peinado et al. (2002, 2005a, 2005b) y del grupo de Física Aplicada de la Universidad de Granada (Kowalski et al., 2003, 2006, 2008). La mayor parte, corresponden a especies arbóreas y solo unos pocos a matorrales. En dichos estudios está representada C. ladanifer pero no E. arborea.

La importancia relativa de los matorrales en su función como sumidero resulta evidenciada de la comparación de los datos obtenidos para Pinus sylvestis L. del monte de Valsaín, con los de la Sierra Suroeste de la provincia de Badajoz: 461,9 $\mathrm{tCO}_{2} /$ ha (Montero et al., 2004) para la especie arbórea y 25,32 $\mathrm{tCO}_{2} /$ ha para los jarales extremeños (Alías et al., 2009). Si comparamos porcentualmente la capacidad de fijación anual, el pinar fija 9,65 $\mathrm{tCO}_{2} / \mathrm{ha} /$ año, mientras que los jarales estudiados 1,26 $\mathrm{tCO}_{2} /$ ha/año. En este caso, lo que se fija por ha y año en los jarales estudiados es un $13 \%$ de lo que fija en el pinar. Por tanto, a pesar de ser cantidades inferiores no deben desestimarse estos porcentajes relativos a ecosistemas y formaciones vegetales a priori más importantes en términos absolutos.

\subsubsection{Combustibles sólidos}

La preocupación por el uso de combustibles fósiles - no renovables - como principal fuente de energía ha ido incrementando a lo largo del tiempo, lo que ha llevado a investigadores, industria y agencias gubernamentales a desarrollar una fuerte investigación sobre el uso de recursos energéticos alternativos y renovables como lo es la biomasa. Obtener energía a través de la biomasa es una de las opciones más prometedoras de energía renovable y uno de los principales objetivos en Europa debido a sus implicaciones ambientales positivas (EU, 2011). Debido a esto, muchos países europeos están desarrollando el uso de pellets o briquetas de madera para el suministro 
de energía a nivel local y muchas industrias están viéndose forzadas a reemplazar los combustibles fósiles por biomasa, neutral en cuanto a emisiones de $\mathrm{CO}_{2}$ y por tanto, creándose un interesante mercado para pellet.

En nuestra región, la jara (C. ladanifer) y el brezo (E. arborea), son dos matorrales muy comunes y atractivos para este mercado incipiente debido al elevado volumen disponible de estas formaciones vegetales, sin embargo, no se suelen usar en la fabricación de pellets. Teniendo en cuenta la problemática que los incendios forestales suponen en España, la posibilidad de prevenir incendios sacando carga de combustible del monte es una de las principales acciones preventivas de incendios, que difícilmente se realiza debido al coste que ello supone, pero que podría ser compensado si esta vegetación arbustiva tuviera un valor comercial. El uso del sotobosque para la fabricación de pellets y briquetas transformará la concepción que se tiene de éste, dejando de ser considerado un problema a eliminar del bosque para convertirse en energía renovable. No obstante, para que la biomasa pueda ser utilizada como una fuente de energía alternativa, sus propiedades fisicoquímicas deben ser caracterizadas y para ello es necesaria más investigación al respecto. Desconociendo estas propiedades, no se puede desarrollar un buen diseño ni una selección óptima de equipos e instalaciones para el tratamiento, almacenamiento y uso de la biomasa.

De los dos arbustos bajo estudio, el que presenta mayor potencial de rentabilidad es el brezo, E. arborea, cuyo poder calorífico ha sido clasificado como el más alto de todas las especies perennes de madera dura del arco mediterráneo (Barboutis y Lykidis, 2014).

Dado que corteza y madera de $C$. ladanifer y de $E$. arborea presentan diferencias en su estructura química, poseerán diferentes propiedades, especialmente en aquellas relacionadas con su potencial aplicación como combustibles. Esta distinción es importante ya que la corteza de las especies perennes de madera dura generalmente presenta mayor contenido en ceniza que la madera, siendo un $2 \%$ el umbral de contenido de ceniza establecido por la Organización Internacional de Normalización en la norma internacional ISO 17225-2:2014, la cual ha sustituido a la norma europea EN 14961-2 sobre pellets de madera para uso no industrial (Consejo Europeo del Pellet, 2011). En la reciente normativa, el poder calorífico neto requerido (NCV) o el poder calorífico inferior (LHV) es $\geq 16.56 \mathrm{~kJ} \cdot \mathrm{g}^{-1}$ y el valor calorífico mayor (HHV) $\geq 18.82$ $\mathrm{kJ} \cdot \mathrm{g}^{-1}$. 


\subsubsection{Precursores de productos de alto valor añadido en Medicina y Cosmética}

\subsubsection{La oleorresina ládano}

El ládano se obtiene al hervir las hojas y las ramas tiernas. Presenta un contenido relativamente alto de ambrox (olor a ámbar) y, por lo tanto, es apreciado en perfumería como sustituto del ámbar gris, procedente del cachalote, especie en peligro de extinción.

En el Génesis (37:25) se indica: "Y levantando los ojos divisaron una caravana de ismaelitas que venían de Galaad, con camellos iban cargados de almáciga, sandáraca, ládano, bálsamo y cáscara resinosa, e iban bajando para llevarlo a Egipto" Los egipcios utilizaron el ládano, en los procesos de embalsamamiento.

También en el Génesis (43:11) está escrito: "Entonces su padre Israel les dijo: Si asi tiene que ser, haced esto: tomad de los mejores productos de la tierra en vuestras vasijas, y llevad a aquel hombre como presente un poco de bálsamo y un poco de miel, resina aromática, mirra, nueces y almendras".

Heródoto de Halicarnaso (484 a.C - 425 a.C), historiador y geógrafo griego; (III, 112): “Aún tiene más de extraño y maravilloso la droga del lédano o ládano como los árabes lo llaman, que nacida en el más hediondo lugar es la que mejor huele de todas, cosa extraña por cierto va criándose en las barbas de las cabras y de los machos de cabríos de donde se les extrae a la manera que del moho de los troncos de los árboles. Es el más provechosos de todos los ungüentos para mil usos, y de él, muy especialmente se sirven los árabes para sus perfumes". De hecho, la bola del ládano recogida directamente en la planta o bien al peinar el pelo de las cabras, recubierta con musgo y ámbar se convirtió, en un codiciado ambientador natural para los árabes.

En el Dioscórides leemos: "Es una mata que crece a la manera del cisto empero produce mas luenga y negras las hojas, sobre las cuales se recoge cierta grasa y unctosa viscosidad, a la prima vera. Untado con vino, disminuye la fealdad de las cicatrizes. Instilado con agua y miel, o con azeyte rosado, sana el dolor de oído".

Según Andrés Laguna: "Esta especie de cisto que aquí describe Dioscórides de la cual se coge el liquor de las boticas llamado ladano, y laudano, es aquella planta muy pegajosa, que en castilla tiene por nombre xara. Escribe Plinio el Viejo que se coge de la yedra cierto liquor, del cual suele hazerse el ladano. Empero aqueste error, como tengo ya declarado, procede de la semejanza de los vocablos, porque cisto nos significa xara y cisso por otra parte yedra, de suerte que tomó el uno por el otro". 
Francisco Suarez de Ribera (1548-1617) lo ilustró como el fruto en polvo de la jara cura la disentería o la diarrea; y el cocimiento de las cortezas cura las hemorragias del aparato respiratorio o la leucorrea, sofocación uterina e incluso cura las encias ulceradas por el escorbuto.

Aunque ya no se usa, el ládano se tomaba en jarabes para la tos. También fue usado en la preparación de linimentos, pomadas y emplastos para el tratamiento de dolores reumáticos. Encontró utilización para combatir síntomas de gastritis y otras patologías del sistema digestivo $\mathrm{y}$, asimismo, fue utilizado como sedante en el insomnio, la ansiedad e histeria. Otra aplicación, ciertamente curiosa, lo fue en el tratamiento de la tosferina: la planta de jara se cortaba y era colgada boca abajo en el dormitorio del enfermo.

\subsubsection{Esencia de jara}

La esencia de jara se obtiene a partir de la destilación de la planta y se convierte en un subproducto muchos más puro y ecológico que la bola de ládano al no tener ningún añadido en su elaboración.

\subsubsection{Aceite de jara}

El aceite de $C$. ladanifer es rico en monoterpenos (67\%) y sesquiterpenos (20\%) y pobre en en diterpenos (3\%). Los componentes principales son $\alpha$-pineno (broncodilatador), trans-pinocarveol, acetato de bornilo, 4-terpineol and camfeno (monoterpenos); viridiflorol (emulador de estrógeno, también encontrado en propolio), ledol y globulol (alcoholes sesquiterpénicos); y 15-nor-labdan-8-ol (diterpeno) (Gomes, 2005; Gülz et al., 1984; Verdeguer et al., 2012).

\subsubsection{Extractos de brezo}

E. arborea contiene muchos compuestos activos, tales como flavonoides, monoterpenos, triterpenoides, glucósidos fenilpropanoides y taninos condensados. Además, extractos de la planta utilizados en Farmacia son considerados antiinflamatorios, antioxidants y analgésicos. De hecho, se considera que en la composición de la corteza (con hojas) se encuentran triterpenoides, tales como friedelin, lupeol, betulina y ácido ursólico (Khassan, 1977; Hegnauer, 1962). 


\subsubsection{Aceite de brezo}

Según Bessah y Benyoussef (2014), el aceite esencial de las hojas de E. arborea de origen argelino y destilado en agua $\left(d=0.8587 ;[\alpha]+2^{\circ} 44^{\prime}\right)$, contiene 75 componentes, entre los que se encuentran como principales constituyentes: el ácido palmítico (33.3\%), el (Z,Z,Z)-9,12,15-octadecatrien-1-ol, un alcohol graso (6.6\%) y el nonacosano, un hidrocarburo de cadena lineal (6.1\%). Otros componentes, presentes en proporciones más bajas, son el alcohol $\beta$-fencílico, $\beta$-cariofileno, $\beta$-bourboneno, eugenol, ionol, geranil acetona and germacreno D.

\subsubsection{Cerveza de brezo}

De aroma intenso, afrutado y floral. Robert Louis Stevenson, en el poema Heather Ale, dedicado a la cerveza de brezo que elaboraban los pictos, aparecen los siguientes versos:

$$
\begin{aligned}
& \text { «De las campanillas del brezo } \\
& \text { lograron una bebida excelente } \\
& \text { mucho más dulce que la miel } \\
& \text { y más fuerte que el vino...». }
\end{aligned}
$$

\subsubsection{Miel de brezo}

La miel de brezo es muy apreciada por su gusto ácido y aromático. Debido a su riqueza en hidroquinona, es un antiséptico urinario. También sirve para un cuidado preventivo de la próstata y tiene uso contra anemias por su riqueza en hierro.

\subsubsection{Precursores de combustibles líquidos: furfural e hidroximetilfurfural}

Una interesante posibilidad de valorización de la biomasa de las especies arbustivas Cistus ladanifer y Erica arborea pasa por su transformación en biodiesel. De cara a esta valorización, la hemicelulosa es la fracción más prometedora ya que su hidrólisis es más sencilla, facilitando la obtención de los aldehídos furfural e hidroximetilfurfural (precursores de los combustibles líquidos) en condiciones más suaves y mediante procesos relativamente cortos.

En la presentación del estado de conocimiento sobre los bioproductos bajo consideración sólo nos ocuparemos de los antecedentes sobre los procesos de transformación de la biomasa arbustiva a furfural e hidroximetilfurfural, y no al de las reacciones de condensación aldólica e hidrogenación que llevan a los combustibles líquidos. Sin embargo, sí haremos hincapié en los productos derivados de furfural e HMF. 


\subsubsection{Furfural (2-furfuraldehído).}

Es el tercer biocombustible que puede ser directamente utilizado en motores diésel. Viene produciéndose desde 1922 (cuando Quaker Oats obtuvo la patente de su producción) a partir del bagazo de la caña de azúcar, cascarilla de arroz o de biomasa no alimentaria.

Actualmente, se producen 250.000 t/año y constituye, hoy por hoy, el único compuesto químico orgánico insaturado preparado a partir de hidratos de carbono. Su síntesis conlleva la hidrólisis en medio acuoso ácido y a alta temperatura de la hemicelulosa contenida en residuos agrícolas y forestales, el $25 \%$ de cuya composición son polisacáridos de D-xilosa (xilosanos). No obstante, en disolución se encuentran presentes hexosas susceptibles de producir 5-hidroximetil-furfural y posteriormente, el ácido levulínico.

A partir de furfural se producen una variedad de compuestos de interés industrial tales como alcohol furfurílico, furfurilamina, ácidos furoico y furanacrílico, furano y tetrahidrofurano. Constituye, además, una unidad base (building block) para obtener moléculas más complejas tales como difurfuril diamina y ésteres de ácido difurfurildicarboxílico, a partir de los cuales pueden obtenerse monómeros para la fabricación de poliamidas y poliésteres, respectivamente.

La mayor parte del furfural se utiliza como disolvente en el refinado de aceites lubricantes y en la preparación del nylon, junto con el alcohol furfurílico, en condensaciones con formaldehído, fenol, acetona o urea, para obtener resinas con excelentes propiedades para su termoconformado, alta resistencia a la corrosión, bajo peligro de incendio y muy buena resistencia física, que se usan extensamente en la industria de la fundición como machos para moldes de alta calidad.

\subsubsection{Hidroximetilfurfural y derivados.}

El hidroximetilfurfural (HMF) es un derivado de un furano con un grupo alcohol y un grupo aldehído y que se forman por descomposición térmica o deshidratación de los glúcidos. La coloración anaranjada o rojiza que adquiere el melocotón maduro se debe a la formación de HMF a partir de fructosa. La miel reciente contiene 1-5 mg $\mathrm{HMF} / \mathrm{kg}$ de miel y la miel envejecida, hasta $40 \mathrm{mg} / \mathrm{kg}$ miel. Las uvas pasas pueden contener hasta $100 \mathrm{mg} / \mathrm{kg}$ de HMF. A partir del HMF se pueden obtener 4 ácidos dicarboxílicos (maleico, málico, succínico y malónico) y ácido glicólico. De hecho, el grupo de investigación de Tecnologías Avanzadas Aplicadas al Desarrollo Rural 
Sostenible (TADRUS) de la Universidad de Valladolid, es capaz de obtener al 100\% ácido levulínico a partir de fructosa pura. Hoy en día, HMF y ácido levulínico son consideradas especies químicas muy versátiles y compuestos potenciales para la producción de biocombustibles.

El HMF se puede convertir en 2,5-dimetilfurano, que es un líquido biocombustible que se puede utilizar como aditivo para sustituir a los alcoholes. Entre sus derivados se encuentran los ácidos delta-amino levulínico, difenólico, succínico, y derivados como 1,4-butanodiol. Es un precursor del nylon, cauchos sintéticos y plásticos y también del metiltetrahidrofurano (biofuel utilizado como aditivo del petróleo, producido vía hidrogenación del ácido levulínico o del furfural) y del ester levulinato de etilo (aditivo tanto del diésel como del petróleo y producido por esterificación del alcohol con ácido levulínico).

\subsubsection{Precursores para materiales compuestos: microfibras de celulosa como particulados}

Debido al rápido incremento de los problemas ambientales, los polímeros biodegradables y biocompatibles se utilizan cada vez en mayor cantidad. Los bioplásticos nacieron de la inquietud de dar solución a los impactos ambientales generados por los residuos de plástico, y a reducir, a corto y medio plazo, la dependencia del petróleo como recurso no renovable. Un bioplástico se define como el material polimérico fabricado a partir de recursos renovables como azúcares, almidón, celulosa, lactosa, patatas, cereales, melazas y otros, que no es fósil, se degrada con rapidez, se puede comportar y se sintetiza con energía renovable. También se llama así a los sintéticos fabricados a partir de petróleo que son biodegradables. Asimismo, se consideran bioplásticos las mezclas de ambos tipos de polímeros, como las de almidón y policaprolactona, ya comercializados en algunos países avanzados. Es importante destacar que éstos materiales pueden procesarse utilizando técnicas convencionales tales como extrusión y soplado.

Las aplicaciones de estos polímeros son múltiples, desde carcasas de computadoras, televisores, celulares, aparatos de música, y productos como los envases que generan un volumen importante de residuos como las botellas de bebidas, los films para productos frescos y confitería, las bandejas de polímero sobre la base de almidón de maíz solubles en agua, hasta cintas adhesivas de celulosa modificada que puede 
usarse también como recubrimiento de bandejas de celulosa o almidón, films de mezclas de Ecoflex con PLA (ácido láctico del maíz, ya granulado), y almidón para envases de alimentos con atmósfera modificada.

Las propiedades mecánicas de los compuestos pueden cambiar con la composición química de las fibras. Las interfases juegan un rol importante en las propiedades físicas y mecánicas de los compuestos. Una buena adhesión a lo largo de la interfase puede obtenerse a través de un mejor mojado de las fibras por la matriz durante la fabricación y también mediante enlaces químicos entre ambos componentes. La celulosa es un polímero hidrofílico que consiste en una cadena lineal de unidades de anhidroglucosa con enlaces $\beta$-1,4 glucosídicos. La gran cantidad de grupos hidroxilo les otorga a las fibras propiedades hidrofílicas. Cuando estas fibras se usan para reforzar matrices hidrofóbicas, el resultado es una interfase muy pobre y en consecuencia una muy baja resistencia a la humedad y propiedades mecánicas menores a las deseadas. Por lo tanto, los tratamientos químicos de las fibras (celulosa) se vuelven importantes para mejorar la adhesión fibra/matriz y, de esta forma, las propiedades de los materiales compuestos obtenidos. El aumento de la compatibilidad entre la fibra y la matriz no sólo mejora la adhesión interfacial sino también el grado de dispersión del refuerzo dentro de la matriz (haciendo más fuertes las interacciones polímero-fibra que las fibra-fibra que conducen a la aglomeración) que es otro factor determinante de las propiedades finales.

Las micrografias SEM de la celulosa sin tratar suelen mostrar una morfología aglomerada en la que se pueden distinguir micro-fibras que conforman cada fibra individual. En las micrografias de las fibras tratadas se puedo ver que a medida que el tratamiento alcalino se hace más agresivo, el diámetro de las fibras disminuye (debido a la separación de las microfibras) y se dispersan.

En relación con los materiales compuestos, se puede afirmar que la adhesión fibra/matriz (de las fibras seleccionadas), suele mejorar respecto al de las fibras sin tratar. Aun así, las propiedades mecánicas de los compuestos sólo alcanzan valores optimizados cuando las fibras tienen la relación de aspecto suficiente para actuar como refuerzo de la matriz (es decir, cuando actúan como propiamente fibras y no como partículas). Las matrices que se han utilizado para la fabricación de materiales compuestos de celulosa abarcan termoplásticos, termoestables y cemento, principalmente. 
Por otro lado, la parte medular en el desarrollo de este tipo de materiales es mejorar la compatibilidad del material lignocelulósico con las matrices utilizadas, para lo cual, se utiliza una infinidad de métodos de modificación superficial. Estos métodos incluyen tratamientos alcalinos y ácidos y agentes compatibilizantes (i.e., silanos, anhidridomaléico). Todas estas modificaciones superficiales tienen como objetivo final el de mejorar las propiedades del material compuesto desarrollado. Al respecto se han encontrado mejoras en algunas de las propiedades mecánicas de los materiales. Por ejemplo, el módulo de Young aumenta con el contenido de fibras utilizadas, mientras que, la resistencia a la tensión del material compuesto disminuye. La disminución en la resistencia a la tensión puede deberse a que, en los trabajos consultados, se utilizaron fibras lignocelulósicas sin tratamiento, fibras cortas sin tener en cuenta el concepto de longitud crítica en el reforzamiento o fibras con tratamiento ácido únicamente. Otra de las propiedades que ha sido mejorada con las modificaciones superficiales es el esfuerzo de corte interfacial, con mejoras superiores al 375\% de esta propiedad al hacer tratamientos superficiales a la fibra del bagazo de caña en una matriz de poli(estireno) (PS).

Las triquitas o nanowhiskers (CNWs, por sus siglas en inglés) y las microfibras de celulosa representan un gran interés a los científicos y tecnólogos hoy en día, debido a su naturaleza renovable, buenas propiedades mecánicas y una relación longitud/diámetro muy alta, lo que conduce a que presente un área superficial muy grande, en comparación con las fibras tradicionales. El módulo de elasticidad teórico de los CNWs materiales ha sido calculado en 167,5 GPa, por lo que se espera que su capacidad de reforzamiento en matrices poliméricas sea excepcional. Las triquitas de celulosa tienen típicamente longitudes de cien a varios cientos de nanómetros y diámetros de entre 3-20 nm y, junto con las microfibras, son usualmente preparados por hidrólisis de fibras de celulosa. Además, se ha encontrado que en la obtención de las triquitas de celulosa, las dimensiones y la cristalinidad de éstas depende de las condiciones de hidrólisis (temperatura, concentración de ácido y tiempo de hidrólisis, entre otros) y la fuente de celulosa que se haya empleado.

Respecto al tamaño de las triquitas es preciso decir que dependen del origen de la celulosa: si es de origen bacteriano o marino (urochordata, tunicata,...) las triquitas son más largas, mientras que el uso de celulosa de madera nos conduce a triquitas más cortas. 


\section{OBJETIVOS}





\section{OBJETIVOS}

En base a lo expuesto en la introducción, se pretende caracterizar las propiedades físicas y estructurales de dos especies pirófilas de matorral mediterráneo: Cistus ladanifer y Erica arborea, dada su amplia extensión en la Península Ibérica, así como el aprovechamiento que se realiza de las mismas. Para ello, se ha estructurado la Tesis Doctoral como un compendio de varias líneas de estudio individuales, que constan de sus respectivos objetivos específicos y que han sido confeccionados como artículos para su publicación en diversas revistas internacionales especializadas.

De este modo, el primero de los objetivos principales consiste en la cuantificación del carbono contenido y su relación entre las partes aérea y radical (relación root to shoot). Como objetivos secundarios, enumeramos los siguientes:

Separación de la biomasa en fracciones, cuantificación y análisis de su distribución, comparando la fisiología de ambas especies y las diferencias entre ambas.

Cuantificación del porcentaje de carbono y nitrógeno contenidos en cada fracción, especialmente en la raíz debido a su omisión en gran parte de los métodos de estimación de dióxido de carbono fijado por la vegetación.

Análisis del comportamiento de ambas especies como sumideros de carbono y su relevancia en la lucha contra el cambio climático.

El objetivo principal que se persigue en la segunda línea de investigación es la caracterización de la madera de jara y de brezo para el estudio de las posibilidades energéticas que éstas presentan. Los objetivos secundarios se enumeran a continuación:

Correlacionar los cálculos de poder calorífico superior y contenido de cenizas de corteza y madera interna con otros métodos -directos e indirectosutilizados en la bibliografía. 
Analizar los diámetros de las dos especies de matorral que permitirían su valorización como combustibles bajo los requerimientos que establece la normativa europea.

Como tercer objetivo se busca definir las propiedades físicoquímicas de los aceites esenciales de ambos matorrales. El objetivo particular de este estudio se define en el siguiente punto:

Comparación de los componentes químicos principales de las fracciones en que se ha dividido cada planta, entre ambas especies bajo estudio y entre los aceites, gomas y resinas de otras especies con alto valor de mercado.

El propósito del cuarto y quinto objetivos son el fraccionamiento de la biomasa de las dos especies arbustivas para obtener, por una parte, biocompuestos como furfural (base química de herbicidas, fungicidas e insecticidas) e hidroximetilfurfural (biocombustible para transporte) y por otra, microfibras cristalinas de celulosa para uso como particulados/reforzantes de materiales compuestos. Los objetivos particulares vinculados son:

Fraccionamiento y valorización de los componentes de la biomasa. Producción de furfural e hidroximetil furfural.

Estudio de la cristalinidad de microfibras de celulosa para su utilización en materiales compuestos. 
4. Material y Métodos 



\section{MATERIAL Y MÉTODOS}

Se recapitula brevemente el conjunto de métodos seguidos en la elaboración de cada artículo científico.

\subsection{PREPARACIÓN DE MUESTRAS}

Muestras de ambas especies de Cistus ladanifer y Erica arborea fueron recogidas atendiendo a que los individuos seleccionados estuvieran sanos y tuvieran características similares al resto de la población. Posteriormente, se procedió a su apeo y a la extracción de la parte subterránea: ambas partes (aérea y radicular) se separaron mediante una sierra y, siguiendo el procedimiento de Ruiz-Peinado et al. se extrajo el sistema radical con la ayuda de un tractor con un pico acoplado y unas piquetas para completar el trabajo manualmente (Figura 9). Para cada planta, se excavó un círculo alrededor con un diámetro equivalente a dos veces su corona; además del grueso de la raíz, se recogieron aquellas que permanecían en el agujero. Las muestras se trasladaron íntegras a laboratorio (instalaciones de la ETSIIAA de la Universidad de Valladolid, España) donde, valiéndose de un calibre (Figura 9), se separaron por fracciones y pesaron en verde. En el caso de Cistus ladanifer fueron clasificadas en hojas, frutos, ramas finas (con un diámetro comprendido entre 3 y $7 \mathrm{~mm}$ ), materia gruesa (de 7-17 $\mathrm{mm}$ de diámetro) y raíces. Por otro lado, Erica arborea, dada su morfología y la imposibilidad de separación de las hojas, se dividió en cuatro fracciones: hojas con flores y frutos, materia fina $(<1 \mathrm{~cm})$, materia gruesa $(<5 \mathrm{~cm})$ y raíces, de acuerdo con Mello et al. Además, para la valorización de Cistus ladanifer y Erica arborea como combustible se diferenció la madera interna y externa de tallo y raíz (Ver 5.2. Valorization of $C$. ladanifer and E. arborea shrubs for fuel: wood and bark thermal characterization). 

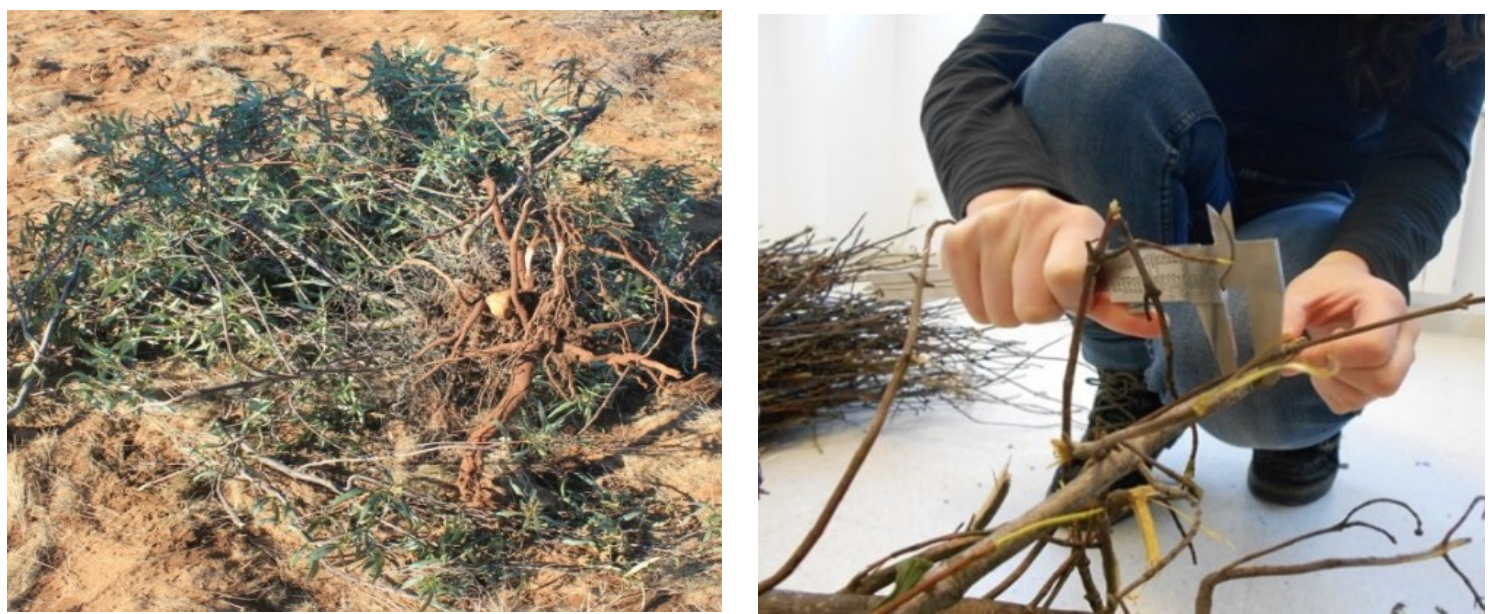

Figura 9. Muestra de jara tras su extracción y proceso de clasificación de las fracciones.

Se determinó empíricamente el contenido de materia seca (biomasa) mediante la extracción de submuestras de cada fracción (Figura 10). Las fracciones de las partes aéreas se secaron en estufa a una temperatura de $102 \pm 2^{\circ} \mathrm{C}$ hasta peso constante, momento en el cual se considera que el contenido de agua es nulo. La parte radical, debido a su envergadura, fue pesada una vez su humedad se equilibró con el ambiente (seco al aire) y se contrastó el resultado con el de una fracción de la misma secada en estufa. Posteriormente, cada fracción se pasó por un molino de cuchillas y otro de bola y se tamizó para obtener polvo de $1 \mathrm{~mm}$ (Figura 10). Los frutos de la jara y los elementos gruesos del tallo requirieron de una prensa hidráulica, dada la resistencia de su estructura al molido.
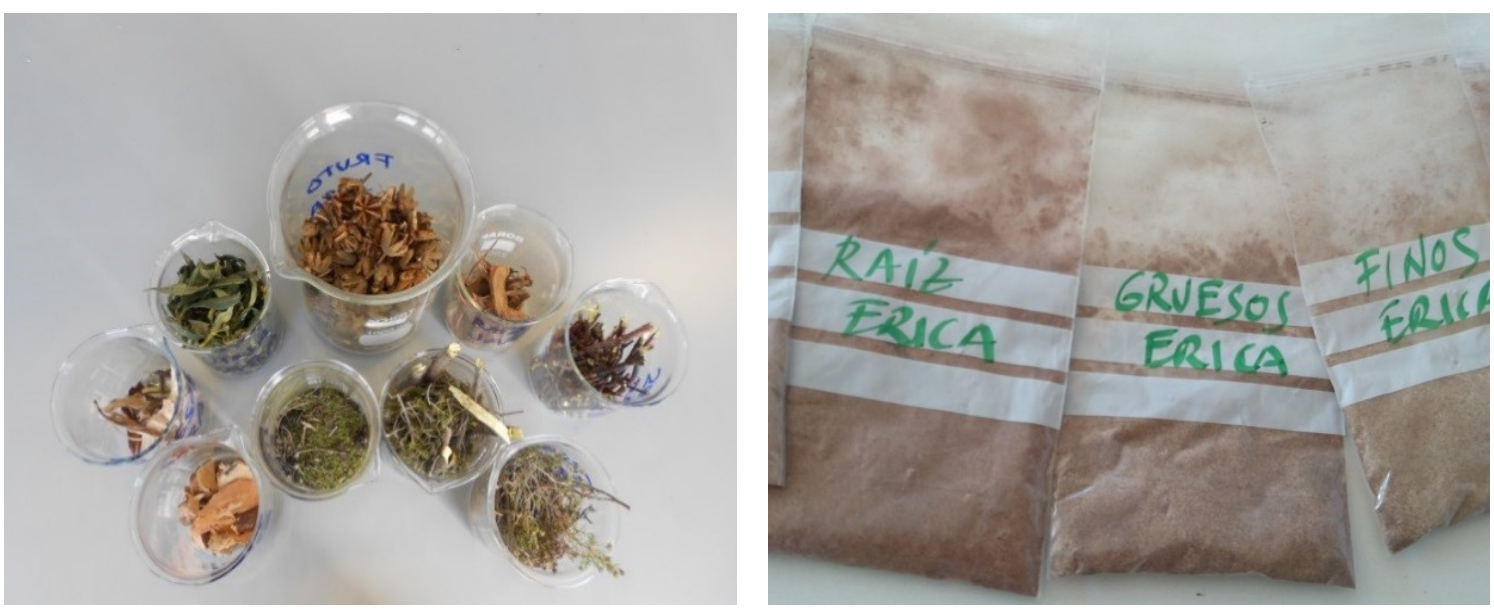

Figura 10. Submuestras seleccionadas antes y después de la pulverización 


\subsection{METODOLOGÍA PARA EL ANÁLISIS DEL MATORRAL MEDITERRÁNEO COMO SUMIDEROS DE CARBONO Y PARA EL CÁLCULO DE NUEVAS RELACIONES ROOT-TO-SHOOT}

La determinación de la concentración tanto de carbono como de nitrógeno se ha determinado con el analizador LECO CHN-2000 (LECO Corp., Saint Joseph, MI, USA). Para el calibrado del analizador se prepararon cuatro repeticiones de 0.09 a 0.12 g de ácido etilendiaminotetraacético al 99\% (CAS No. 60-00-4), adquirido a Alfa Aesar (Thermo Fisher (Kandel) GmbH, Karlsruhe, Germany); mientras que el peso de las muestras de jara y brezo se mantiene constante, $0.10 \mathrm{~g}$ en la báscula de precisión. Se envuelven de forma individual en láminas de estaño y se les da forma de esfera, posteriormente se colocan en un muestreador automático que las va introduciendo en el equipo. El sistema automatizado realiza la combustión de las muestras a una temperatura de $900^{\circ} \mathrm{C}$, tras lo cual recoge los productos restantes de la combustión $\left(\mathrm{CO}_{2}, \mathrm{H}_{2} \mathrm{O}, \mathrm{O}_{2}, \mathrm{~N}_{2}\right.$ and $\left.\mathrm{NO}_{\mathrm{x}}\right)$. Los niveles de $\mathrm{CO}_{2}$ y $\mathrm{H}_{2} \mathrm{O}$ se monitorizan con dos detectores infrarrojos - selectivos y no dispersivos-independientes, y el $\mathrm{N}_{2}$ se determina mediante un detector de conductividad térmica. El aparato ofrece de forma directa los resultados con el peso compensado, dando el porcentaje de contenido de carbono y nitrógeno de cada fracción (Figura 11).
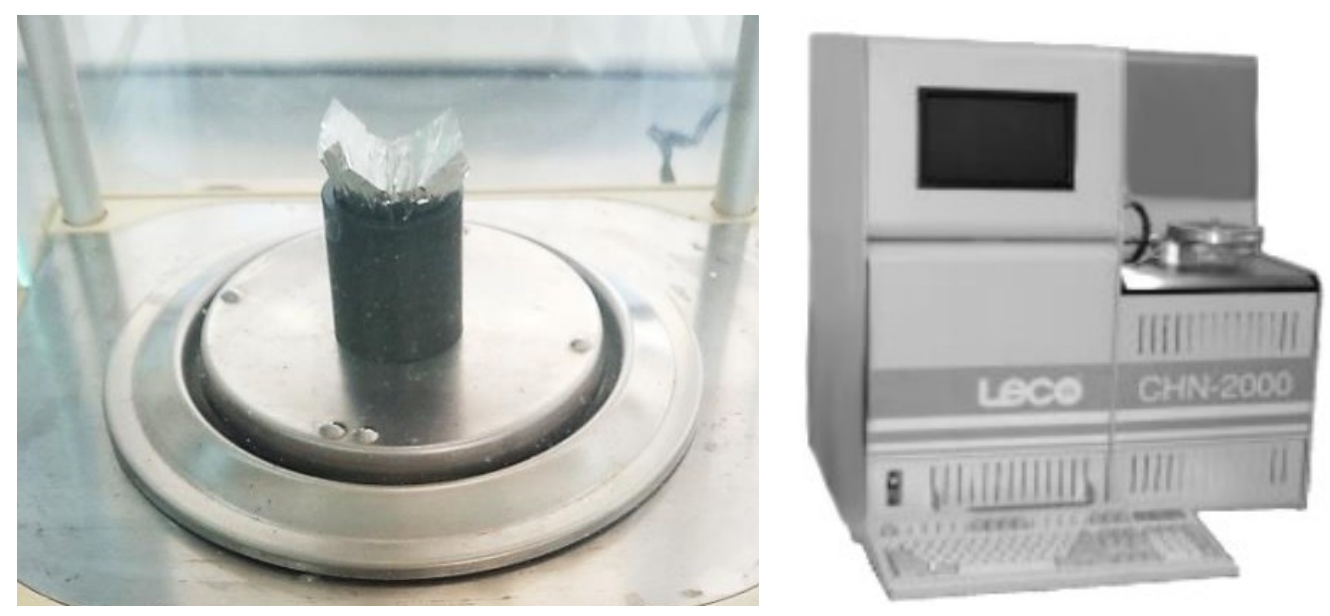

Figura 11. Lámina de estaño en balanza de precisión y equipo LECO CHN-2000

El ratio root-to-shoot $(R)$ fue definido en 2006 por el IPCC, siendo la relación entre la biomasa de la parte subterránea o radical (root) y la de la parte aérea (shoot). Se ha considerado la parte aérea como el sumatorio de hojas, ramas finas y materia gruesa. Las ecuaciones para obtener el valor de $R$ pueden variar de proyecto a proyecto, variando según especies, fase de crecimiento y el lugar, por lo que los cálculos se han 
realizado, al igual que Sanquetta et al., bajo condiciones específicas e idénticas para las muestras de las dos especies consideradas.

La espectroscopía infrarroja con transformada de Fourier (FTIR) es una técnica analítica para la caracterización no destructiva de muestras biológicas. Se ha recogido el espectro FTIR en transmitancia directa de hojas, materia fina, materia gruesa y raíces de C. ladanifer y E. arborea, utilizando un espectrómetro Thermo Nicolet iS50 (Thermo Fisher Scientific, Waltham, MA, USA) y aplicando el método de pellets de bromuro potásico (KBr). Éstos se obtenían mezclando $1.0 \mathrm{wt} \%$ de muestra en $200 \mathrm{mg}$ de $\mathrm{KBr}$ en polvo e introduciéndolo en un troquel específico para la formación de los pellets de 13 $\mathrm{mm}$ de diámetro; se forman en una prensa, aplicando el vacío con una fuerza de aproximadamente 8 toneladas durante varios minutos (Figura 12).
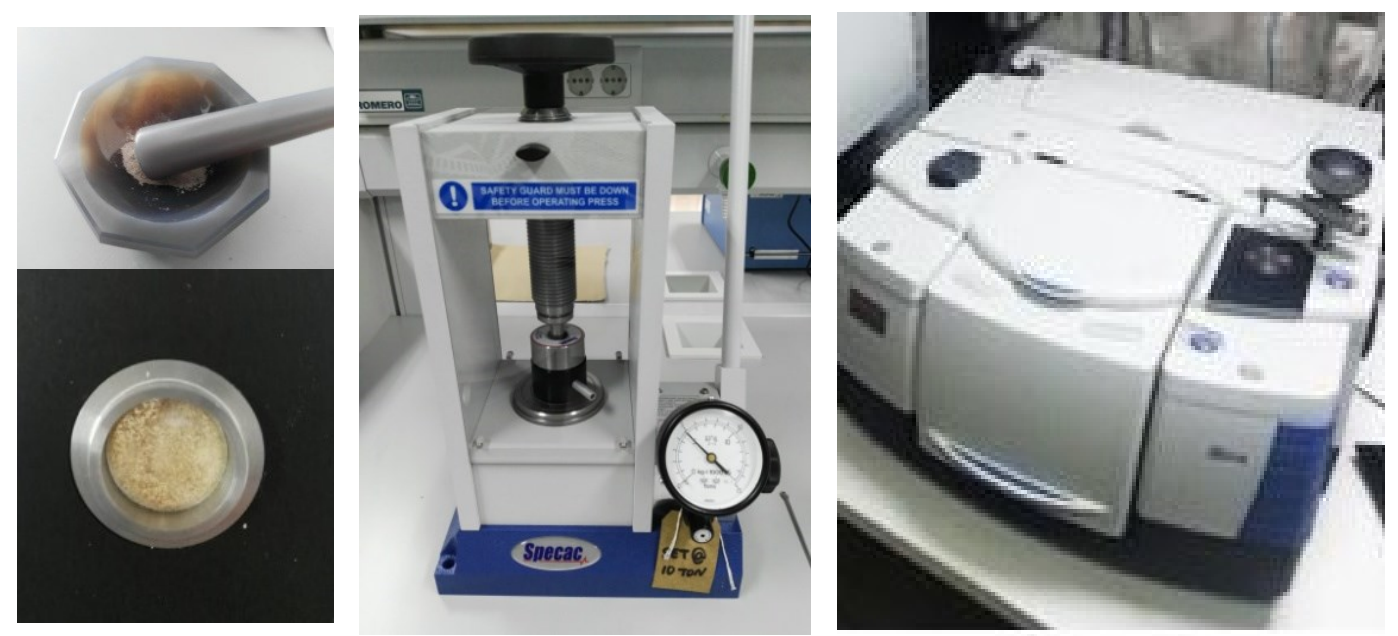

Figura 12. Preparación de una muestra en mortero tras su pesado (extremo superior izquierda), ejemplo de pastilla de muestra KBr (izquierda inferior), prensa utilizada en la formación de los pellets y equipo utilizado

Los espectros se recogieron en el rango del infrarrojo medio $\left(4000-400 \mathrm{~cm}^{-1}\right)$ con una resolución espectral de $4 \mathrm{~cm}^{-1}$ y tomando 32 lecturas por muestra. Se realizaban escáneres denominados background en intervalos de 60 min para un ajuste adecuado y la corrección de los registros. Para ello se utilizó una pastilla de KBr puro, para así corregir tanto las pérdidas por dispersión de la luz infrarroja en cada pastilla como la humedad absorbida por el $\mathrm{KBr}$ (Figura 12). La información vibracional fue analizada con el software OMNIC v.9.3.32 (Thermo Fisher Scientific), enfocando el estudio en la región de la huella dactilar $\left(1900-800 \mathrm{~cm}^{-1}\right)$, puesto que es donde suceden la mayor parte de las variaciones de la absorción infrarroja. 


\subsection{METODOLOGÍA PARA LA VALORIZACIÓN DE CISTUS LADANIFER Y ERICA ARBOREA COMO COMBUSTIBLES SÓLIDOS: CARACTERIZACIÓN TÉRMICA DE MADERA Y CORTEZA}

Se ha determinado el poder calorífico superior llevando de nuevo todos los productos derivados de la combustión a la temperatura previa a la misma, y condensando el vapor producido. Equivale al calor de combustión en termodinámica ya que el cambio de entalpía debido a la reacción conlleva que la temperatura previa y posterior a la combustión de los componentes sea la misma; por lo tanto, el agua generada en la combustión se condensa a estado líquido, produciendo su calor latente de vaporización.

La estimación de biomasa y de los valores caloríficos correspondientes pueden obtenerse mediante métodos directos e indirectos; mientras que los métodos directos implican la destrucción de la biomasa, los indirectos utilizan ecuaciones para estimar los valores caloríficos a través de las mediciones de otras variables (Bombelli et al. 2009).

Los valores caloríficos han sido determinados mediante un método directo, implicando la selección, tala y extracción de la biomasa de cada una de las especies, con su consecuente combustión (Figura 13). A partir de las muestras recolectadas y correspondientemente separadas por fracciones (Ver 4.1. Preparación de muestras), se ha determinado para cada especie el diámetro del tallo, el grosor de la corteza y los porcentajes de madera y corteza. Tras la separación de la corteza del tallo de madera y su correspondiente medición, se trituraron las muestras con una astilladora portátil. En el caso de C. ladanifer, se seleccionaron dos grupos de individuos atendiendo al diámetro medio del tallo y la edad, de modo que hubiera en la muestra diámetros superiores a la media de ejemplares mayores y más robustos, con el fin de tener mayor probabilidad de cumplir los requisitos de la norma ISO 17225-2 para contenido en cenizas (ENplus-B class).

Los valores caloríficos de las fracciones se calcularon mediante metodologías diferentes, tanto indirectamente aplicando ecuaciones ya estandarizadas como empíricamente. En primer lugar, el alto poder calorífico se calculó a través del análisis elemental de las muestras de ambas especies, siguiendo la normativa del Instituto americano de Tecnología del GAS (IGT) propuesta por Talwalkar et al. en 1981; posteriormente se calculó aplicando los porcentajes de holocelulosa y de la mezcla de lignina y extractos aplicando los factores propuestos por Aseeva et al. (2005) y Kienzle 
et al. (2001) y, finalmente, de manera experimental en un calorímetro de bomba Par 1261 de isoperibol (Thermo Fisher Scientific, Waltham, MA, EE.UU.) siguiendo los procedimientos señalados en la norma BS EN 14918:2009.

Además, también se ha calculado experimentalmente la entalpía total de combustión mediante calorimetría de barrido diferencial (DSC) y análisis termogravimétrico con termoanálisis diferencial.
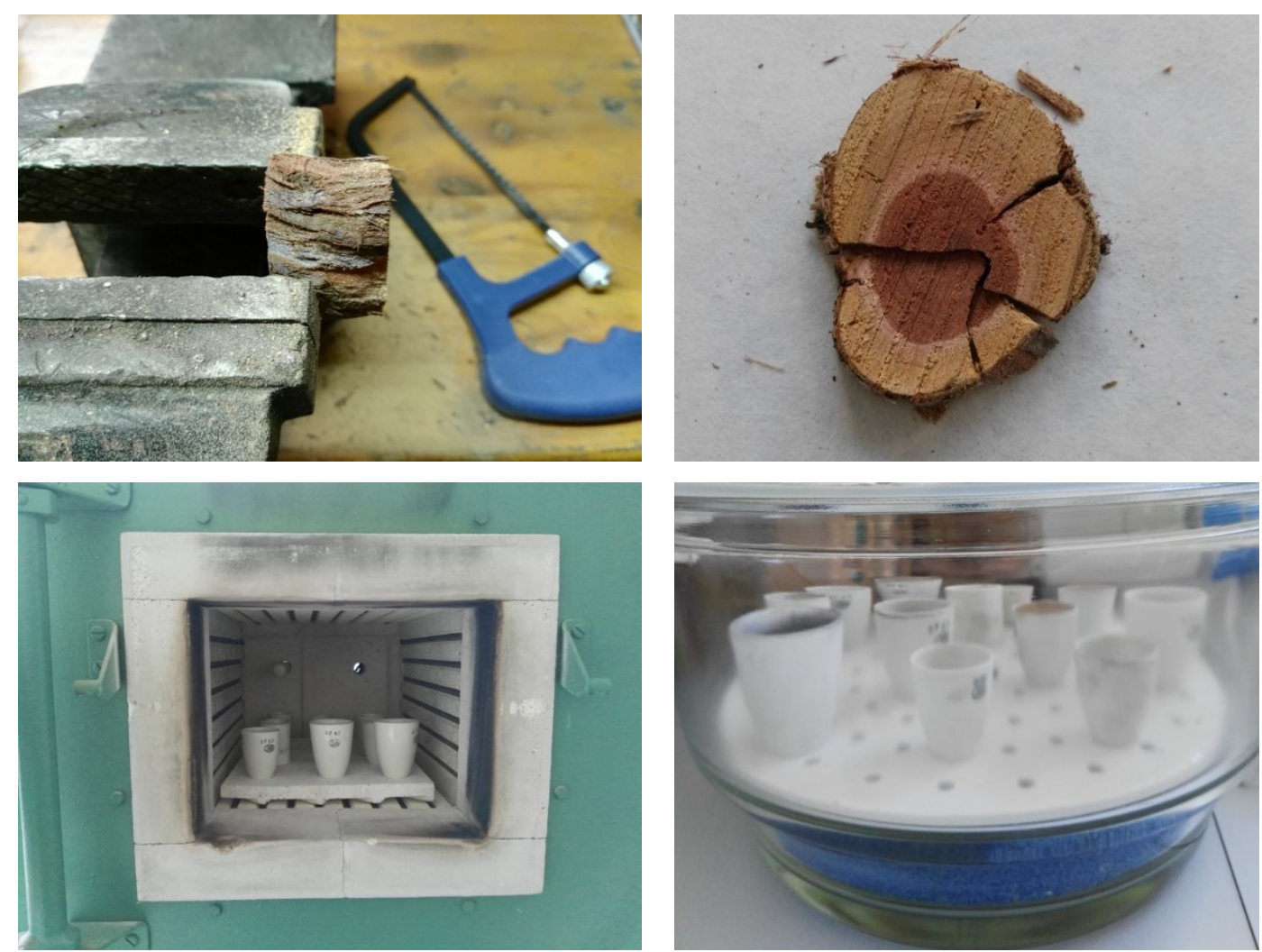

Figura 13. Proceso seguido en la determinación del contenido de cenizas, siguiendo la metodología descrita en ISO 18122:2015. En el extremo superior izquierdo, un fragmento del tallo previo al serrado, a su derecha, una muestra de los discos obtenidos; en el extremo inferior izquierdo, crisoles en mufla y a su derecha, en el desecador 


\subsection{METODOLOGÍA PARA EL ESTUDIO VIBRACIONAL Y TÉRMICO DE LOS ACEITES ESENCIALES DE CISTUS LADANIFER Y ERICA ARBOREA}

La extracción de los aceites esenciales se llevó a cabo mediante la hidrodestilación de $100 \mathrm{~g}$ de hojas frescas de C. ladanifer y de E. arborea (Figura 14), se recogieron, secaron bajo sulfato anhidro de sodio y almacenaron hasta su utilización. A su vez, de forma alternativa se extrajeron suspendiendo la materia vegetal seca en alcohol amílico. No obstante, para confirmar su pureza, se determinó la composición por cromatografía de gases acoplada a un espectrómetro de masas (GC-MS).

En cambio, los aceites de Rosa rubiginosa L. y de Elaeis guineensis Jacq., la mirra del género Commiphora, masilla de Pistacea lentiscus L. y goma tragacanto de Astragalus, utilizados como comparación, se obtuvieron de manera comercial; al igual que una muestra de aceite esencial de E. arborea.

Se estudiaron los espectros de vibración en el infrarrojo medio a través de un espectrómetro con transformada de Fourier equipado con un sistema de reflexión total atenuada (ATR), los análisis termogravimétricos (TG) y calorimétricos de barrido diferencial (DSC) - a alta temperatura - en un autoanalizador térmico simultáneo y, los registros DSC a baja temperatura en un calorímetro de compensación de energía.

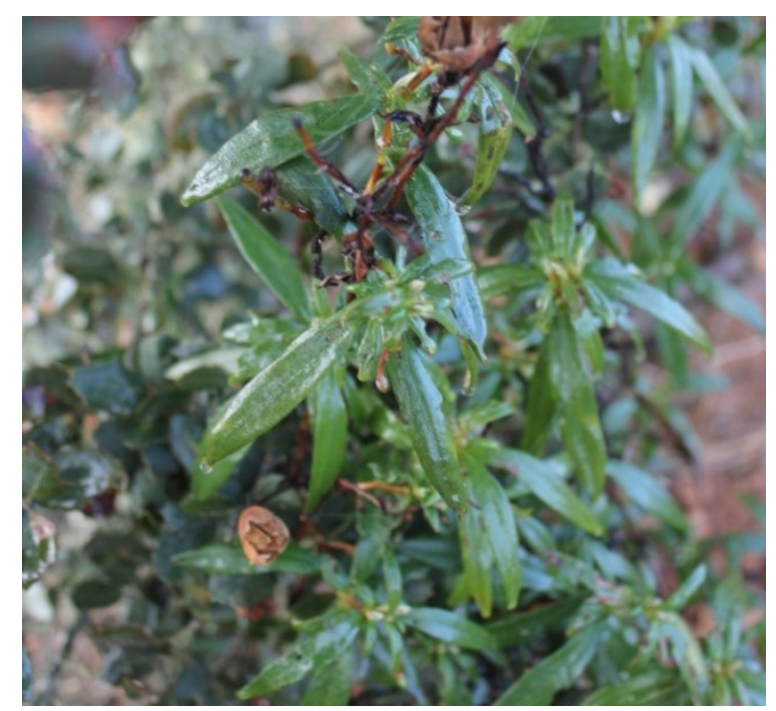

Figura 14. Muestra de la exudación de C. ladanifer en campo 


\subsection{METODOLOGÍA PARA EL FRACCIONAMIENTO DE LA BIOMASA DE CISTUS LADANIFER Y ERICA ARBOREA Y SU CONVERSIÓN A PRODUCTOS CON VALOR AÑADIDO. CARACTERIZACIÓN DE AZÚCARES, LIGNINA, FURFURAL, 5-HIDROXIMETIL FURFURAL Y MICROFIBRAS CRISTALINAS DE CELULOSA}

El material objeto de estudio son distintas fracciones de la biomasa de Cistus ladanifer y Erica arborea: corteza de tallo, tallo interno, tallo externo, corteza de raíz, raíz interna y raíz externa, con sus respectivas réplicas.

En primer lugar, se separan las huminas dada su insolubilidad en medio alcalino. Para ello, se preparan las disoluciones con $20 \mathrm{mg}$ de cada muestra y $2 \mathrm{~mL}$ de $\mathrm{NaOH} 0.1$ M, dejándolas $24 \mathrm{~h}$ en un agitador (Figura 15). Para obtener las huminas, se decanta y lava el sólido obtenido (material A). Del residuo correspondiente a las muestras de tallo de ambas especies (las de mayor interés para la obtención de microfibras de celulosa) se han obtenido imágenes por microscopia electrónica de barrido (SEM).
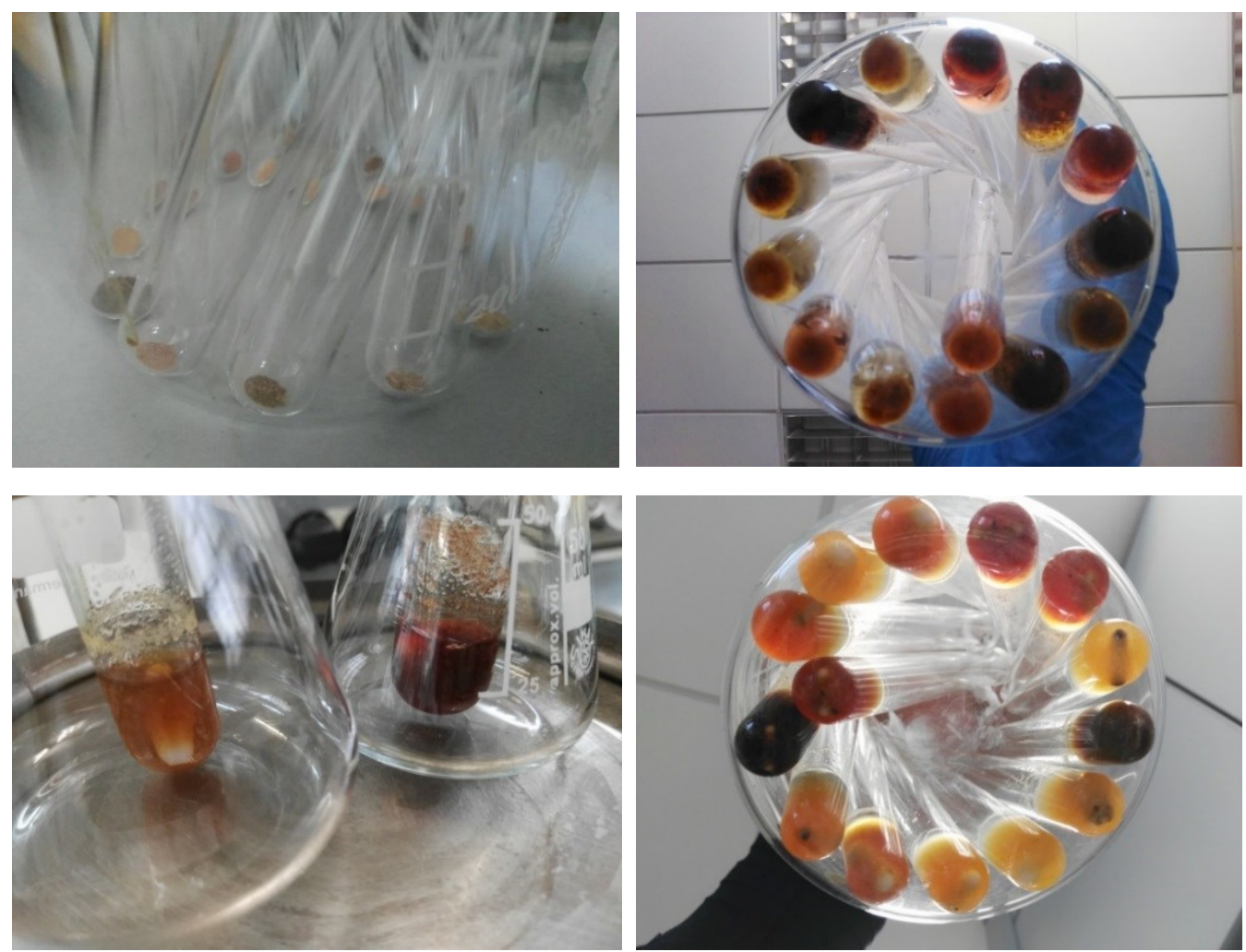

Figura 15. Secuencia del tratamiento con NaOH: pesado de muestras, adición de la base, proceso de agitación con la ayuda de imanes y resultado tras la digestión 
De las disoluciones anteriores se extraen $0.3 \mathrm{~mL}$ por muestra y se diluyen en matraces de $10 \mathrm{~mL}$, aunque al ser la concentración elevada para su registro en el espectrofotómetro, se llevaron a $25 \mathrm{~mL}$ (Figura 18). El pH debe ser neutro, por lo que se debe neutralizar con $\mathrm{HCl}$ si así fuese necesario. Si al añadirlo aparece precipitado, la muestra contiene ácidos húmicos, que, al ser insolubles en medio ácido, precipitan. En cambio, los ácidos fúlvicos sí son solubles (material B). Para la obtención del patrón se emplea glucosa, preparándose varias disoluciones de distintas concentraciones que abarquen el rango de absorciones más amplio posible (30-200 mg).

Para el tratamiento de hidrolización de la biomasa del material $\mathrm{B}$, se han seguido las siguientes metodologías:

En disolvente eutéctico profundo (Deep eutectic solvent, DES) con digestión asistida por microondas. Para la hidrólisis de la biomasa de C. ladanifer y E. arborea se utilizó un disolvente eutéctico profundo, un líquido iónico con base de cloruro de colina-urea (Figuras 16 y 17). Se preparó agitando la mezcla de cloruro de colina y la urea (relación molar de 1:2) a $80^{\circ} \mathrm{C}$ hasta que se formara un líquido incoloro homogéneo, para almacenarlo después en un desecador al vacío. Posteriormente, se añadió a las muestras de biomasa (200 mg) cloruro de colina/urea y ácido nítrico al 10\% $(8 \mathrm{~mL})$, con óxido de titanio como catalizador $(20 \mathrm{mg})$ y se trataron en un sistema de digestión por microondas - horno microondas Ethos-One de la casa Milestone (Sorisole, BG, Italy), equipado con un sistema de agitador magnético- a $120{ }^{\circ} \mathrm{C}$ para un tiempo comprendido entre 1 y 60 min. Las muestras se dejaron enfriar a temperatura ambiente. A continuación, el DES se eliminó mediante lavado con agua.

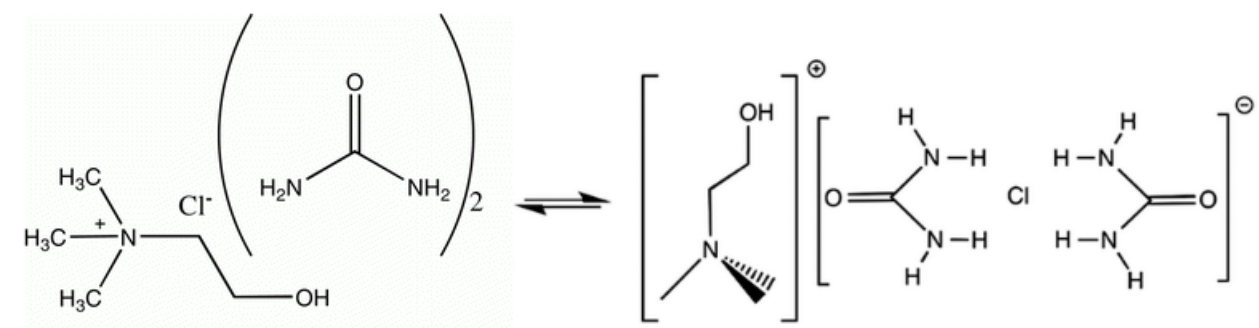

Figura 16. DES a base de cloruro de colina y urea donde el cation [colina] ${ }^{+}$es energéticamente energético con $\left[\mathrm{Cl}(\text { urea })_{2}\right]^{-}$

En disolvente polar aprótico DMAc, con bicarbonato de sodio y aplicación de microondas. Como método alternativo al anterior, se utilizaron $8 \mathrm{~mL}$ de N,Ndimetilacetamida (DMAc), un líquido de alto punto de ebullición, incoloro y miscible en agua, con $40 \mathrm{mg}$ de hidrógenocarbonato de sodio $\left(\mathrm{NaHCO}_{3}\right)$ como agente para el 
tratamiento de la madera de C. ladanifer y E. arborea, siendo sendas muestras de biomasa de $200 \mathrm{mg}$. Se aplicó el mismo procedimiento que con el disolvente anterior: se le aplicaron microondas con un agitador magnético a $120{ }^{\circ} \mathrm{C}$ entre 1 y $60 \mathrm{~min}$, las muestras se dejaron enfriar a temperatura ambiente y se eliminó la DMAc mediante lavado con agua.

En DMAc como disolventey metóxido de potasio asistido con microondas. Este tercer método surge como tercer recurso electivo. El tratamiento hidrolítico de $200 \mathrm{mg}$ de biomasa de $C$. ladanifer y $E$. arborea consistió en una mezcla de $8 \mathrm{~mL}$ de DMAc y $40 \mathrm{mg}$ de metóxido de potasio (utilizado comúnmente como catalizador en la transesterificación en la producción de biodiesel). El ion metóxido se preparó en laboratorio haciendo reaccionar metanol e hidróxido. Tras ello, se trató la solución como en los métodos previos.
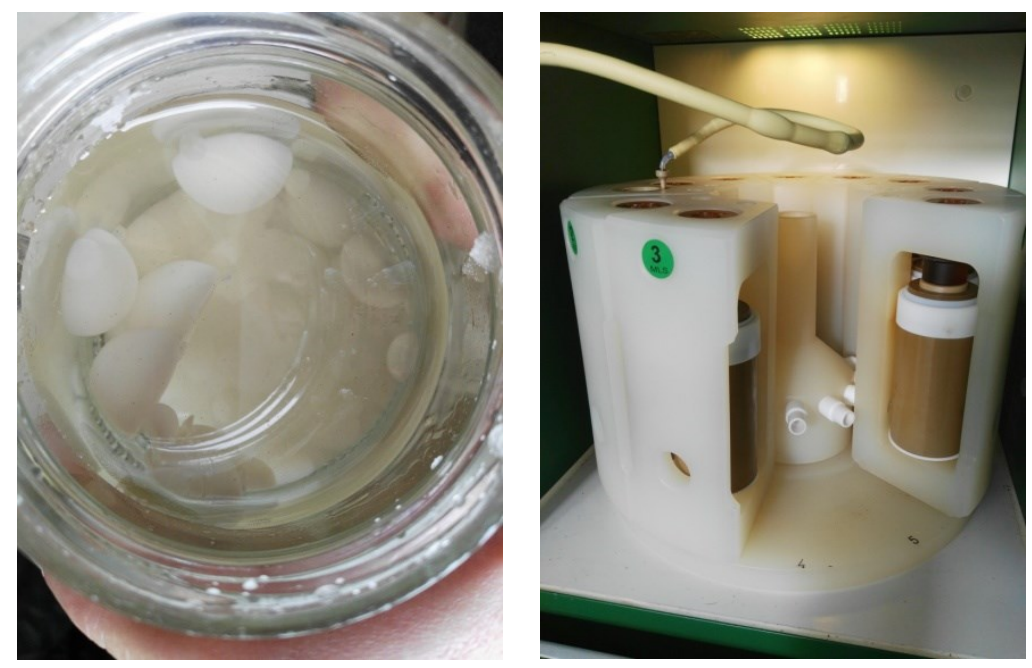

Figura 17. DES cristalizado a temperatura ambiente y preparación de muestras en horno microondas

\subsubsection{Métodos de determinación de azúcares, lignina, furfural e hidroximetilfurfural}

La cuantificación de azúcares reductores se ha realizado mediante el método de Miller utilizando el ácido 3-amino 5-nitrosalicílico (DNS) como reactivo más específico de una disolución que contiene hidróxido sódico y tartrato sódico potásico (Figura 18). La disolución se prepara mezclando $0.8 \mathrm{~g}$ de $\mathrm{NaOH}, 15 \mathrm{~g}$ de tartrato sódico potásico y $0.5 \mathrm{~g}$ de DNS y se completa con agua destilada hasta $50 \mathrm{~mL}$. Para asegurar su homogeneidad, se la mantiene 5 minutos en ebullición. Después, es enfriada con agua y hielo, se le añaden $5 \mathrm{~mL}$ de agua para compensar el volumen evaporado y se deja 15 min de reposo. Con esta disolución, que llamaremos DNS, se preparan mezclas 1:1 con las muestras y disoluciones patrón a analizar: usualmente, $0.5 \mathrm{~mL}$ de DNS y $0.5 \mathrm{~mL}$, 
tanto de cada muestra como a las disoluciones patrón. La determinación de azúcares reductores en estas mezclas se realiza a través de la medición de sus absorbancias por lectura espectrofotométrica a $540 \mathrm{~nm}$ (rango visible del espectro).

La determinación de azúcares totales se ha llevado a cabo por el método de Dubois et al. (Figura 18). Este método es llamado fenol-sulfúrico porque en la preparación de las soluciones de medida se mezclan, en tubos de ensayo, 1-2 mL de muestra, $1 \mathrm{~mL}$ de fenol (5\%) y $5 \mathrm{~mL}$ de ácido sulfúrico concentrado (95.5\%). Los tubos de ensayo que contienen estas soluciones se colocan en una gradilla que se mantiene en baño termostatado, entre $25-30{ }^{\circ} \mathrm{C}$, durante $10-15$ min. Como muestra patrón, se ha utilizado glucosa a distintas concentraciones. La medida espectrofotométrica de azúcares totales se realiza a $490 \mathrm{~nm}$ (rango visible del espectro), longitud de onda a la que las hexosas y sus derivados metilados presentan absorción máxima.

El contenido de azúcares no reductores fue calculado por diferencia entre los porcentajes de azúcares totales y azúcares reductores.
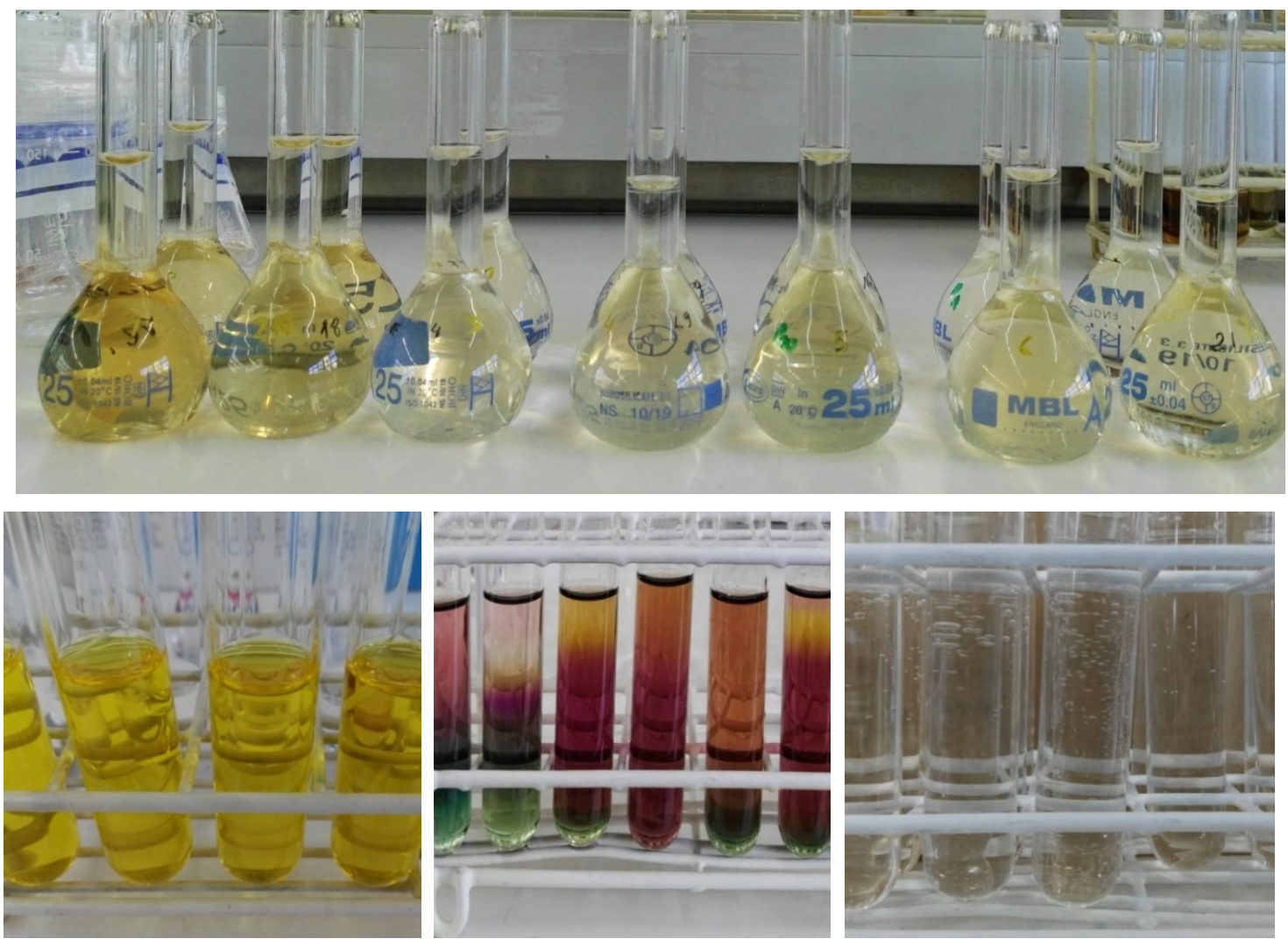

Figura 18. Extracto de la disolución en $\mathrm{NaOH}$ en matraces para su registro espectrofotomético (imagen superior); distintas coloraciones tras los métodos descritos, siendo Miller, Dubois et al. y Chi et al. de izquierda a derecha, respectivamente (imágenes inferiores). 
Para determinar lignina ácida soluble, furfural e hidroximetil furfural (HMF), se ha seguido la metodología utilizada por Chi et al. (2009) con borohidruro como reductor (Figura 18). En primer lugar, se analizan los ácidos fúlvicos en la región ultravioleta del espectro entre 200 y $400 \mathrm{~nm}$ con el fin de obtener las relaciones E2:E3, utilizadas en la estimación de la aromaticidad y el peso molecular, E2:E4 como medida de la humificación y E4:E6, como medida para determinar el carácter del carbón orgánico disuelto (Peacock et al, 2014). Después de ello, se adicionan $30 \mathrm{mg}$ de borohidruro de sodio y tras $5 \mathrm{~min}$, una pequeña cantidad de $\mathrm{HCl}$. Las muestras patrón consideradas son lignina, furfural y HMF, que presentan absorbancias máximas a longitudes de onda de $205 \mathrm{~nm}, 277 \mathrm{~nm}$ y $285 \mathrm{~nm}$, respectivamente. No obstante, si las concentraciones de furfural e hidroximetilfurfural son elevadas, resulta más idóneo medir la lignina ácida soluble a $280 \mathrm{~nm}$. El valor de la lignina será el registro previo a la reducción de borohidruro, mientras que el del furfural y HMF será el de la diferencia entre el valor previo y el posterior.

\subsubsection{Tratamiento de cristalinización de la celulosa y medidas de accesibilidad y cristalinidad}

Muestras de tallo de C. ladanifer y de E. arborea $(200 \mathrm{mg})$, secadas previamente a $70{ }^{\circ} \mathrm{C}$, fueron trituradas en un molino de cuchillas y se sumergieron en $4 \mathrm{~mL}$ de una solución de $\mathrm{NaOH}(25 \% \mathrm{p} / \mathrm{v})$ durante $24 \mathrm{~h}$ a temperatura ambiente con agitación magnética vigorosa. Posteriormente, se lavaron las fibras alcalinizadas, primero con agua corriente y más tarde con agua destilada hasta eliminar toda presencia alcalina en el lavado y finalmente, fueron dejadas en un desecador al vacío hasta tener un peso constante. El lavado alcalino fue reciclado para evitar contaminación ambiental.

Alternativamente, también se utilizó un líquido iónico, un disolvente eutéctico profundo (DES) con base de cloruro de colina y urea para la fibrilación de la celulosa de la madera de $C$. ladanifer y E. arborea. Se preparó el DES de cloruro de colina/urea agitando la mezcla de cloruro de colina y la urea (relación molar de 1:2) a $80{ }^{\circ} \mathrm{C}$ hasta que se formara un líquido incoloro homogéneo, para almacenarlo después en un desecador al vacío.

Posteriormente, las muestras de celulosa $(200 \mathrm{mg})$ fueron tratadas con una mezcla de cloruro de colina/urea y $\mathrm{HNO}_{3}(1.8 \mathrm{~mL})$ y $\mathrm{TiO}_{2}$ como catalizador $(20 \mathrm{mg})$ en un sistema de digestión por microondas —en horno microondas Ethos-One de la casa Milestone (Sorisole, BG, Italy), equipado con un sistema de agitador magnético- a 
$120^{\circ} \mathrm{C}$ durante $30 \mathrm{~min}$. Las muestras se dejaron enfriar a temperatura ambiente. A continuación, el DES se eliminó mediante lavado con agua.

Para determinar la cristalinidad a través de difracción de rayos X (XRPD), se utilizó un difractrómetro D8 Advance Bragg-Brentano de Bruker (Billerica, MA, USA) en geometría de reflexión empleando radiación $\mathrm{Cu} K_{\alpha}(\lambda=1.5406 \AA$ ). Los difractogramas se recogieron mediante exploración continua en el intervalo $2 \theta=5-80^{\circ}$. El índice de cristalinidad $(C r I)$ se calculó a través de la ecuación de Segal et al., $C r I=[1$ $\left.I_{\mathrm{am}} / I_{002}\right] \times 100$, donde $I_{002}$ es la intensidad máxima de la dispersión cristalina en la reflexión $002\left(2 \theta=22.3^{\circ}\right.$ en la celulosa) y $I_{\mathrm{am}}$ se corresponde con la intensidad mínima o "valle" entre los picos a $2 \theta=18^{\circ}$ y $22^{\circ}$. El índice de amorficidad $(A m I)$ es, evidentemente, $A m I=1-C r I$.

Los espectros vibracionales de los materiales fueron analizados en el rango 400$4000 \mathrm{~cm}^{-1}$ mediante un espectrómetro Nicolet iS50 FT-IR, de Thermo Scientific (Waltham, MA, USA), equipado con un sistema incorporado de reflexión total atenuada (ATR) con diamante. Las bandas de absorción a 1430 y $890 \mathrm{~cm}^{-1}$ fueron las utilizadas en el cálculo del índice de orden lateral (lateral order index, $L O I$ ).

Las micrografías SEM fueron tomadas con un microscopio Quanta 200FEG de FEI (Hillsboro, OR, USA) con detectores de electrones retrodispersados y emitidos (BSE y S, equipado con un espectrómetro Genesis dispersivo de energía de rayos $\mathrm{X}$ (EDS). Las muestras analizadas no han sido metalizadas.

Las mediciones de absorción de yodo se llevaron a cabo para comprobar la accesibilidad de la celulosa, según el método ASTM D4607-94. Se calculó el ISV o valor de absorción de yodo (mg) mediante la ecuación $I S V=[(a-b) \times c \times 254] / w$, donde $a$ es el volumen de solución de hiposulfito de sodio control $(\mathrm{mL})$ utilizado, $b$ es el volumen de solución de hiposulfito de sodio consumido con las muestras (mL), $c$ es la concentración molar de hiposulfito de sodio ( $\mathrm{mol} / \mathrm{L}), \mathrm{y} w$ es el peso seco de la muestra (g). 

5.RESULTADOS 

5.1. Publicación \#1:

\section{MEDITERRANEAN SHRUBLANDS AS CARBON SINKS FOR} CLIMATE CHANGE MITIGATION: NEW ROOT TO SHOOT RATIOS (CARBON MANAGEMENT, 2017. DOI: 10.1080/17583004.2017.1285178)

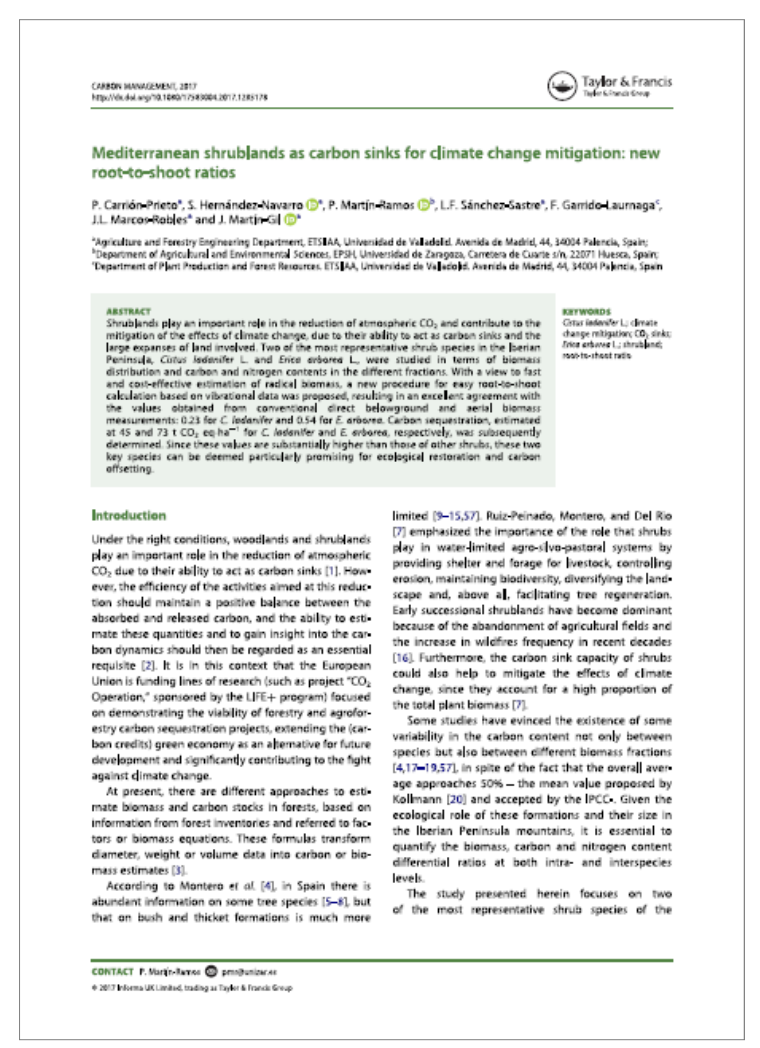





\title{
Mediterranean shrublands as carbon sinks for Climate Change mitigation: new root to shoot ratios
}

\author{
P. Carrión-Prieto ${ }^{a}$, S. Hernández-Navarro ${ }^{a}$, P. Martín-Ramos ${ }^{b *}$, L.F. Sánchez-Sastre ${ }^{a}$, \\ F. Garrido-Laurnaga ${ }^{c}$, J.L. Marcos-Robles ${ }^{\mathrm{a}}$ and J. Martín-Gil ${ }^{a}$ \\ a Agriculture and Forestry Engineering Department, ETSIIAA, Universidad de \\ Valladolid. Avenida de Madrid, 44, 34004 Palencia, Spain. Phone: +34 (979) \\ 108347; Fax: +34 (979) 108301; E-mail:paula.carrion@alumnos.uva.es; \\ inpaisal@iaf.uva.es; luxpher@gmail.com; jlmarcos@iaf.uva.es; mgil@iaf.uva.es. \\ $b$ Department of Agricultural and Environmental Sciences, EPSH, Universidad de \\ Zaragoza, Carretera de Cuarte s/n, 22071 Huesca, Spain. Phone: +34 (974) \\ 292668; Fax: +34 (974) 239302;E-mail:pmr@unizar.es. \\ $c$ Department of Plant Production and Forest Resources. ETSIIAA, Universidad de \\ Valladolid. Avenida de Madrid, 44, 34004 Palencia, Spain. Phone: +34 (979) \\ 108370; Fax: +34 (979) 108301; E-mail:fegala@pvs.uva.es.
}

(Received 29 October 2016; accepted XX)

\section{Funding}

This work was supported by the European Union LIFE+ Programme under Grant LIFE1 1 ENV/ES/000535 ("CO 2 Operation: Integrated agroforestry practices and nature conservation against climate change").

\begin{abstract}
Shrublands play an important role in the reduction of atmospheric $\mathrm{CO}_{2}$ and contribute to the mitigation of the effects of Climate Change, due to their ability to act as carbon sinks and the large expanses of land involved. Two of the most representative shrub species in the Iberian Peninsula, Cistus ladanifer L. and Erica arborea L., were studied in terms of biomass distribution and carbon and nitrogen contents in the different fractions. With a view to fast and cost-effective estimation of radical biomass, a new procedure for facile root-to-shoot calculation based on vibrational data was proposed, resulting in an excellent agreement with the values obtained from conventional direct belowground and aerial biomass measurements: 0.23 for $C$. ladanifer and 0.54 for $E$. arborea. Carbon sequestration, estimated at 45 and $73 \mathrm{t} \mathrm{CO}_{2} \mathrm{eq} \cdot \mathrm{ha}^{-1}$ for $C$. ladanider and $E$. arborea, respectively, was subsequently determined. Since these values are substantially higher than those of other shrubs, these two key species can be deemed as particularly promising for ecological restoration and carbon offsetting.
\end{abstract}

Keywords: Cistus ladanifer L.; Climate Change mitigation; $\mathrm{CO}_{2}$ sinks; Erica arborea L.; shrubland; root to shoot ratio.

${ }^{*}$ Corresponding author. Email: pmr@unizar.es 


\section{Introduction}

Under the right conditions, woodlands and shrublands play an important role in the reduction of atmospheric $\mathrm{CO}_{2}$ due to their ability to act as carbon sinks (Watson and Noble 2002). However, the efficiency of the activities aimed at this reduction should maintain a positive balance between the absorbed and released carbon, and the ability to estimate these quantities and to gain insight into the carbon dynamics should then be regarded as essential requisites (OrellanaRivadeneyra et al. 2012). It is in this context, the European Union is funding lines of research (such as project " $\mathrm{CO}_{2}$ Operation", sponsored by LIFE+ program) focused on demonstrating the viability of forestry and agroforestry carbon sequestration projects, extending the (carbon credits) green economy as an alternative for future development and significantly contributing to the fight against Climate Change.

At present, there are different approaches to estimate biomass and carbon stocks in forests, based on information from forest inventories and referred to factors or biomass equations. These formulas transform diameter, weight or volume data into carbon or biomass estimates (Somogyi et al. 2007).

According to Montero, Pasalodos-Tato, Montoto, et al. (2013), in Spain there is abundant information on some tree species (Ibàñez, Vayreda, and Gracia 2002, Montero, Ruiz-Peinado, and Muñoz 2005, RuizPeinado, del Rio, and Montero 2011, RuizPeinado, Montero, and Del Rio 2012), but that on bushes and thickets formations is much more limited (Alías et al. 2015, García Rosa 2013, Blanco Oyonarte and Navarro Cerrillo 2003, Castro et al. 1996, Navarro 2004, Cerrillo and Oyonarte 2006, MendozaPonce and Galicia 2010, Paton et al. 1997). Ruiz-Peinado, Montero, and Del Rio (2012) emphasized the importance of the role that shrubs play in water-limited agro-silvopastoral systems by providing shelter and forage for livestock, controlling erosion, maintaining biodiversity, diversifying the landscape, and above all, facilitating tree regeneration. Early successional shrublands have become dominant because of the abandonment of agricultural fields and the increase in wildfires frequency in recent decades (Masalles and Vigo 1987). Furthermore, the carbon sink capacity of shrubs could also help to mitigate the effects of Climate Change, since they account for a high proportion of the total plant biomass (Ruiz-Peinado, Montero, and Del Rio 2012).

Some studies have evinced the existence of some variability in the carbon content not only between species but also between different biomass fractions (Montero, Pasalodos-Tato, Montoto, et al. 2013, García Rosa 2013, Gayoso A and Guerra C 2005, Lin, Liu, and Lin 2002, Alías Gallego et al. 2009), in spite of the fact that the overall average approaches 50\% -the mean value proposed by Kollmann (1959) and accepted by the IPCC-. Given the ecological role of these formations and their size in the Iberian Peninsula mountains, it is essential to quantify the biomass, carbon and nitrogen content differential ratios both at intra- and interspecies levels.

The study presented herein focuses on two of the most representative shrub species of the Iberian Peninsula, namely Cistus ladanifer L. and Erica arborea L. (Figure 1), which occupy surfaces of over $2,100,000$ ha and 2,400,000 ha, respectively (MAPAMA 2016). They have a wide distribution, according to the Spanish Forest Map (MAPAMA 2006) and the Anthos Spanish Plants Information System (Spanish Biodiversity Foundation and Spanish Royal Botanic Garden 2011), and also appear accompanying tree species, in such a way that they have their own codification in the Spanish National Forest Inventory (MAPAMA 2016). Phytosociologically, these species are very important in pure Mediterranean shrublands, in siliceous soils (Serrada, Montero, and Reque Kilchenmann 2008), and Cistus ladanifer has a relevant role as an animal feed source (Serrada, Montero, and Reque Kilchenmann 2008). These species are represented in various habitats of the Council Directive 92/43/EEC. 
Fractions of these two species, grown under the same conditions, have been assessed, placing particular emphasis on an accurate determination of their radical biomass, provided that the literature tends to underestimate root:shoot ratios $(R)$ according to Mokany, Raison, and Prokushkin (2006). The aim of this work has been to obtain these ratios, since they are an expansion factor used for inferring belowground biomass from aboveground biomass measurements (IPCC 2006) and, provided that it is based on biomass and carbon, it can provide ecological values for the calculation of stock, production and ecosystem productivity that are closer to reality.

[FIG. 1]

\section{Material and methods}

\section{Location}

The study was carried out on a plot located in the municipality of Ayoó de Vidriales $\left(42^{\circ}\right.$ $\left.07^{\prime} 10^{\prime \prime} \mathrm{N}, 6^{\circ} 06^{\prime} 59^{\prime \prime} \mathrm{W}\right)$, in the province of Zamora, Castilla y Leon, Spain (Figure 2). The chosen area $(>1.2 \mathrm{ha})$ is a mixed shrubland in which the dominant shrub species are Erica arborea L. (Ea) and Cistus ladanifer L. $(\mathrm{Cl})$.

With a continental climate -typical of the northern plateau of the Iberian Peninsula-, temperatures are extreme, with monthly average temperature values ranging from -2 ${ }^{\circ} \mathrm{C}$ to $25^{\circ} \mathrm{C}$. Rainfall is scarce (about $440 \mathrm{~mm}$ per year), with a pronounced drought period from late May to mid-September. The soil belongs to Inceptisols (i.e., soils of relatively new origin, characterized by having only the weakest appearance of horizons, or layers, produced by soil-forming factors), suborder Ochrept (i.e., it is a young soil with thin, light colored horizons), with a xeric moisture regime (Xerochept).

[FIG. 2]

\section{Data sampling and fresh weight determination}

Calculations for the estimation of biomass and carbon stocks may be obtained either by direct or by indirect methods (Sanquetta et al. 2004). Direct methods involve the destruction of heavy biomass, whereas in indirect methods regression models are used to estimate stored biomass and carbon from measurements of other variables -such as DBH (diameter at breast height), tree height $(\mathrm{H})$ or age-, making the process easier (Sanquetta, Corte, and da Silva 2011).

In the first part of this study, biomass was determined by a destructive method, which comprised the selection, felling and extraction of biomass for each of the species (conducted in December 2013). Selected samples corresponded to healthy individuals and featured similar characteristics to the rest of the population. The aerial part was separated from the roots using a saw and then, following an analogous procedure to that described by Ruiz-Peinado, Montero, and Del Rio (2012), root systems were excavated by using a tractor with a shovel and then spades were used to complete the job. For each plant, soil was excavated down in a circular area of twice the mean crown diameter. In addition to the main body of the roots, those remaining in the hole were also collected.

25 samples of each species were transported to the laboratory (ETSIIAA facilities, Universidad de Valladolid, Spain), where they were separated into different fractions and weighed (fresh weight). In the case of Cistus ladanifer, they were classified into leaves, fruits, thin branches $(3-7 \mathrm{~mm}$ in diameter), thick branches (7-17 $\mathrm{mm}$ in diameter) and roots. On the other hand, for Erica arborea -given its morphology and the impracticality of leaves separation- they were divided into four fractions: leaves with flowers and fruits, fine material $(<1 \mathrm{~cm})$, thick material $(<5 \mathrm{~cm})$ and roots, in agreement with de Mello et al. (2012).

\section{Dry matter content}

The dry matter (biomass) content was empirically determined by extracting 
subsamples from each fraction. The fractions of the aerial parts were dried in oven at $102 \pm 2^{\circ} \mathrm{C}$ until constant weight was attained (at which the water content was assumed to be zero). The roots, because of their size, were weighted once their moisture was balanced with the environment (i.e., air-dried) and the results were crosschecked by comparison with those obtained for some fractions dried in the stove. The dry matter content for each component was calculated, in agreement with de Mello et al. (2012), using the following expression:

$$
\text { Dry matter }(\%)=\frac{\mathrm{w}_{\mathrm{dry}}}{\mathrm{w}_{\text {fresh }}} \cdot 100
$$

where $W_{d r y}$ is the dry weight $(\mathrm{g})$ and $W_{\text {fresh }}$ is the fresh weight (g).

Subsequently, each fraction was ground in a ball mill and homogenized to obtain $1 \mathrm{~mm}$ sieve powder (the fruits of the gum rockrose and some thick stem elements required a hydraulic press, given the resistance of the structure to grinding) for $\mathrm{CHN}$ analysis (discussed below).

\section{Root-to-shoot ratio as indicator of the relationship between the belowground and aerial biomass}

Root-to-shoot ratios can be applied to individual plants or to stands of vegetation at a local, landscape, regional or biome scale (Mokany, Raison, and Prokushkin 2006), and they are often considered as constant or species/area specific values in most studies (Wang, Duan, and Zhang 2011). The root-toshoot ratio is defined by the IPCC (2006) as the ratio of belowground (root) to aboveground (shoot) biomass -including leaves, thin branches and thick branches-, as follows:

$$
\mathrm{R}=\frac{\mathrm{W}_{\text {root }}}{\mathrm{W}_{\text {shoot }}}
$$

where $R$ is the root-to-shoot ratio (dimensionless), $W_{\text {root }}$ is the root dry weight (g) and $W_{\text {shoot }}$ is the aboveground dry weight (g).

The use of the root-to-shoot ratio as an indicator of the relationship between the belowground (root biomass) and aerial biomass (the sum of leaves, thin branches and thick branches biomasses) is particularly important, since it can serve as an estimator of belowground carbon based on a simple biometric survey of aboveground biomass with lower costs (Schenk and Jackson 2002). Consequently, realistic root-to-shoot ratios play a key role in the improvement of the accuracy of estimates of root biomass and, in turn, in the estimation of the effects of management and land-use changes in national inventories of greenhouse gas emissions (Mokany, Raison, and Prokushkin 2006).

The equations for the obtaining of the $R$ value may vary from project to project. Individual standard values are frequently used, such as those proposed by Kauppi, Mielikainen, and Kuusela (1992), Kauppi, Tomppo, and Ferm (1995), Löwe, Seufert, and Raes (2000), UN-ECE/FAO (2000), Federici et al. (2015) and IPCC (2006). Nevertheless, it is known that these factors also vary depending on the species, the growth stage and the location: the $R$ biomass ratio of adult plants in Mediterranean ecosystems tends to be higher than in more temperate ecosystems, possibly as an adaptation to the summer dry season (Hilbert 1990, Lloret, Casanovas, and Penuelas 1999). Consequently, calculations were performed under specific and identical conditions for the samples of the two species under study, in agreement with Sanquetta, Corte, and da Silva (2011).

\section{FTIR spectroscopy as new tool to determine root-to-shoot ratios}

Fourier transform infrared (FTIR) spectroscopy is a useful analytical technique for the nondestructive characterization of biological specimens. It is regarded as a rapid and accurate method for the fast and simultaneous qualitative and quantitative characterization of natural products and their constituents (Huck 2015). Molecular bonds with an electric dipole moment that can change by atomic displacement owing to natural vibrations are IR active. These vibrational modes are quantitatively 
measurable by FTIR spectroscopy (Griffiths and Haseth 2007, Baker et al. 2014).

The FTIR spectra of leaves, thin branches, thick branches and roots of $C$. ladanifer and $E$. arborea were collected in direct transmittance mode using a Thermo Nicolet iS50 spectrometer (Thermo Fisher Scientific, Waltham, MA, USA) and the potassium bromide (KBr) pellet method. $13 \mathrm{~mm}$ diameter pellets were obtained by mixing 1.0 $\mathrm{wt} \%$ sample into $200 \mathrm{mg}$ of fine $\mathrm{KBr}$ powder and then finely pulverizing and putting into a pellet-forming die. A force of approximately 8 tons was applied under a vacuum of several $\mathrm{mm} \mathrm{Hg}$ for several minutes to form transparent pellets. Spectra were recorded in the mid-infrared range $\left(4000-400 \mathrm{~cm}^{-1}\right)$ at a spectral resolution of $4 \mathrm{~cm}^{-1}$, taking 32 scans per sample. Background scanning and correction was carried out at 60 min intervals, using a pure $\mathrm{KBr}$ pellet for the background spectra to correct for infrared light scattering losses in the pellet and for moisture adsorbed on the KBr (Evans 1991, Owen and Thomas 1989, Chen et al. 2010).

The vibrational data were analyzed with OMNIC v.9.3.32 (Thermo Fisher Scientific) software, focusing on the fingerprint region (1900-800 $\left.\mathrm{cm}^{-1}\right)$, in which most of the variations of infrared absorption occur. Within the fingerprint region, four wavenumbers were selected for the calculation of the shoot-to-root ratios: 1369 $\mathrm{cm}^{-1}$, attributable to the $\mathrm{C}-\mathrm{H}$ and $\mathrm{C}-\mathrm{O}$ groups of the hexose ring in cellulose; $1458 \mathrm{~cm}^{-1}$, consistent with the saccharide backbone; 1514 $\mathrm{cm}^{-1}$, attributed to the $\mathrm{C}=\mathrm{C}$ stretching vibration in the aromatic skeletal vibration in lignin; and $1730 \mathrm{~cm}^{-1}$, assigned to ester linkage of the carboxylic group in hemicelluloses.

Root-to-shoot ratios for both species, $C$. ladanifer and $E$. arborea, based on vibrational data were calculated using the corrected peak areas at the four wavenumbers indicated above and equation 3 :

$$
\underset{\text { roots }}{\mathrm{R}_{\lambda}}=\frac{\mathrm{A}_{\text {root }}}{\mathrm{A}_{\text {shoot }}}=
$$

$\overline{\text { (leafs+thin branches+thick branches) }}$ where $R_{\lambda}$ is the root-to-shoot ratio (dimensionless) for each wavenumber; $A_{\text {root }}$ is the area of the peak in the roots sample; and $A_{\text {shoot }}$ is the aboveground peak area, i.e., the summation of the peak areas in the leaves, thin branches and thick branches samples. As noted above, these ratios are often regarded as constant or species/area specific values (Wang, Duan, and Zhang 2011).

It should be clarified that the peak height depends on the number of molecules present (concentration) and on the strength of the absorption (absorptivity). Conversely, the area of the peak is regarded as a better indicator of concentration, because the final peak profile is the sum of all the individual elements. Whereas in some cases the peak height can be changed by a broadening problem, the area will remain unchanged, as the total number of molecules is constant.

The corrected areas (defined as the areas under the spectrum bordered with a baseline) in the spectral regions of interest were determined with OMNIC software, using the automatic baseline correction procedure prior to area calculation. Figure 3 shows how the areas at each selected wavenumber were calculated, taking the fingerprint region of the vibrational spectrum of $E$. arborea leaves as an example.

\section{[FIG. 3]}

\section{Carbon and nitrogen determination in the laboratory}

The determination of the carbon and nitrogen concentrations was conducted using a LECO CHN-2000 analyzer (LECO Corp., Saint Joseph, MI, USA). Ethylenediaminetetraacetic acid, 99\%; CAS No. 60-00-4), purchased from Alfa Aesar (Thermo Fisher (Kandel) GmbH, Karlsruhe, Germany), was used for the analyzer calibration in four replicates from 0.09 to 0.12 $\mathrm{g}$, while the weight of the samples of the two shrubs under study was always $0.10 \mathrm{~g}$ (measured in a precision scale). Samples were individually wrapped in tin foil and shaped into spheres and, subsequently, they were 
placed in an autosampler that loaded them into the apparatus. The automated system performed the combustion of samples at a temperature of $900{ }^{\circ} \mathrm{C}$ and the remaining products of combustion $\left(\mathrm{CO}_{2}, \mathrm{H}_{2} \mathrm{O}, \mathrm{O}_{2}, \mathrm{~N}_{2}\right.$ and $\mathrm{NO}_{\mathrm{x}}$ ) were collected and mixed thoroughly. $\mathrm{CO}_{2}$ and $\mathrm{H}_{2} \mathrm{O}$ levels were monitored by two independent selective nondispersive infrared detectors, and $\mathrm{N}_{2}$ was determined by a thermal conductivity detector. The apparatus directly provided the weight-compensated results as a percentage of carbon and nitrogen content in each fraction.

There are many studies about the allocation of nitrogen in the plants, provided that it changes as a function of the species and time of the year. However, there is a strong linear relationship between plant nitrogen concentration and the fraction of mass allocated to leaves (Hilbert 1990, Agren and Ingestad 1987):

$$
P \cdot S=\frac{d N}{d t}=a N
$$

where $P$ is the net photosynthesis, $S$ is the fraction of mass allocated to leaves, $N$ is the nitrogen concentration, and $a$ is a constant.

\section{Indirect calculation of carbon stock}

Aboveground biomass was estimated from the equations proposed by Montero, Pasalodos-Tato, López-Senespleda, et al. (2013) for Mediterranean shrublands. These equations, deemed as accurate for large expanses of territory, are based on data from the canopy cover and average height of different species of Mediterranean shrublands and provide tons of dry matter per hectare, differing according to taxonomic affinities. The species analyzed herein belong to the formations classified as 'gum rockroses and Cistaceae shrublands' [tr.] and 'briar roots and Ericaceae shrublands' [tr.], and their respective equations are as follows:

$$
\begin{gathered}
\ln \left(\mathrm{W}_{\mathrm{a} \mathrm{Cl}}\right)=-2.596+0.957 . \\
\ln \left(\mathrm{H}_{\mathrm{av} \mathrm{Cl}}\right)+0.747 \cdot \ln \left(\mathrm{FCC}_{\mathrm{av} \mathrm{Cl}}\right) \\
{[\text { Eq. 5] }} \\
\ln \left(\mathrm{W}_{\mathrm{a} \mathrm{Ea}}\right)=-2.921+0.984 \cdot \ln \left(\mathrm{H}_{\mathrm{av} \mathrm{Ea}}\right)+ \\
0.863 \cdot \ln \left(\mathrm{FCC}_{\mathrm{av} \mathrm{Ea}}\right) \\
{[\text { Eq. } 6]}
\end{gathered}
$$

where $W_{a}$ is the amount of aboveground biomass, in tons of dry matter per hectare (tons DW $\cdot \mathrm{ha}^{-1}$ ); $H_{a v}$ stands for the average height of the shrub expressed in decimeters (dm); and $F C C_{a v}$ represents the canopy cover of the shrub expressed in $\%$.

The root biomasses of each of the shrub species were estimated by applying the value of the root-to-shoot ratios determined in previous sections to the aboveground biomass calculated for each hectare of shrubland. The average height was measured by a sample inventory with a range pole, and the canopy cover was determined through GIS (Geographic Information Systems) tools, using LARS (Low Altitude Remote Sensing) data collected with a remotely piloted aircraft (RPA).

The total amount of carbon stored was predicted using the experimentally determined carbon content of each fraction. Thus, the tons of carbon per hectare of shrubland were estimated using equation 7 :

$$
\begin{gathered}
\mathrm{C}_{\mathrm{t}}=\left[\mathrm{W}_{\mathrm{a} \mathrm{Cl}} \cdot\left(\mathrm{CC}_{\text {shoot Cl}}+\mathrm{R}_{\mathrm{Cl}} \cdot \mathrm{CC}_{\text {root Cl }}\right)+\right. \\
\left.\mathrm{W}_{\mathrm{a} \mathrm{Ea}} \cdot\left(\mathrm{CC}_{\text {shoot Ea }}+\mathrm{R}_{\mathrm{Ea}} \cdot \mathrm{CC}_{\text {root Ea }}\right)\right] \cdot \mathrm{S}
\end{gathered}
$$

[Eq. 7]

where $C_{t}$ is the total assimilated carbon (tons), $W_{a}$ is the aboveground biomass (tons $\cdot \mathrm{ha}^{-1}$ ), $R$ is the root-to-shoot ratio, $C C$ is the amount of carbon absorbed by each fraction of biomass $(\%)$, and $S$ is the surface (ha).

\section{Results and discussion}

\section{Biomass distribution and root-to-shoot ratios}

Figure 4 shows the biomass distribution in each plant: $C$. ladanifer has 19\% of leaves, $1 \%$ of capsules, $29 \%$ of thin branches, $33 \%$ of thick branches and $18 \%$ of roots; versus $E$. arborea with $4 \%$ of leaves, $20 \%$ of thin branches, $41 \%$ of thick branches and $35 \%$ of roots. Thus, in C. ladanifer the aboveground biomass accounted for $3.98 \mathrm{~g}$, roughly $81.8 \%$ of the total dry mass, whereas belowground (roots) accounted for $0.89 \mathrm{~g}, 18.2 \%$. On the other hand, in E. arborea root biomass (8.06 g) represented $35.3 \%$ of the total dry mass, while the remaining $64.6 \% \quad(14.72 \mathrm{~g})$ corresponded to aboveground biomass. 
Consequently, the root-to-shoot ratios, calculated as the quotients of 18.2 by 81.8 and 35.8 by 64.6 , were $R=0.22$ for $C$. ladanifer and $R=0.55$ for E. arborea.

The distribution of biomass -and therefore of carbon uptake- differed in the two shrub species under study: whereas for $C$. ladanifer biomass and assimilated carbon were roughly similar in each of the components that the plant was divided in, for E. arborea significant differences were observed amongst the various fractions. This becomes evident in view of the values of $R$ : while the aerial part of $C$. ladanifer accounted for over $80 \%$ of the dry weight of the plant, in E. arborea it was ca. $65 \%$. This can be ascribed to the characteristics of the shrubs under study, such as the labdanum present in the leaves of the Cistaceae or the development of a root capable of holding the arboreal freightage of the Ericaceae. Moreover, it should also be taken into consideration that, under such environmental conditions, phenology reflects the strategy of plants to cope with the alternation of favourable and unfavourable seasons for assimilation and growth (CastroDíez, Montserrat-martí, and Cornelissen 2003).

\section{[FIG. 4]}

\section{Root-to-shoot ratios based on vibrational analysis}

The infrared spectra of the different fractions of the two shrubs under study, $C$. ladanifer and E. arborea, are depicted in Figure 5. The main absorption bands and their assignments are listed in Table 1.

\section{[FIG. 5] [TABLE 1]}

In the fingerprint region, four wavenumbers were selected: $1369 \mathrm{~cm}^{-1}$, attributable to in-plane bending vibrations of the $\mathrm{C}-\mathrm{H}$ and $\mathrm{C}-\mathrm{O}$ groups of the hexose ring in the cellulose; $1458 \mathrm{~cm}^{-1}$, associated with the alkane deformation of $\mathrm{CH}$ and $\mathrm{CH}_{2}$ in the saccharide backbone; $1514 \mathrm{~cm}^{-1}$, ascribed to the $\mathrm{C}=\mathrm{C}$ stretching vibration in the aromatic skeletal vibration in lignin; and $1730 \mathrm{~cm}^{-1}$, assigned to the ester linkage of the carboxylic group of ferulic and p-coumaric acids in hemicelluloses. The corrected area values for these selected peaks are summarized in Table 2. In general terms, peak areas for E. arborea were higher than those of $C$. ladanifer, in particular those associated to thick branches and roots (which are richer in lignin for $E$. arborea, Table 3 ).

\section{[TABLE 2] [TABLE 3]}

Peak areas at $1369 \mathrm{~cm}^{-1}$ and at $1514 \mathrm{~cm}^{-1}$ were lower than those at $1730 \mathrm{~cm}^{-1}$ at 1458 $\mathrm{cm}^{-1}$. For the peak at $1369 \mathrm{~cm}^{-1}$, leaves and thin branches showed higher area values than those of roots and thick branches, due to their higher cellulose content. In Erica arborea, as regards the peak at $1514 \mathrm{~cm}^{-1}$, thick branches and roots showed peak areas higher than those of leaves and thin branches, due the higher content of lignin of the former (mature wood has high condensed lignin structures with higher molecular weight than younger tissues) (Amir et al. 2006). Conversely, for the peak at $1730 \mathrm{~cm}^{-1}$ thin branches showed the highest area values because of their higher content in hemicellulose. In relation to this latter peak, which is a hemicellulose content indicator, the areas for both the thin and thick branches proved to be the sum of the areas under the three other peaks: $6.22 \approx \sum(1.30+3.97+1.26)$; $3.90 \approx \sum(0.86+2.66+0.93)$;

$5.98 \approx \sum(1.20+3.04+1.03)$

$4.77 \approx \sum(1.75+2.60+0.94)$.

Regarding the $1458 \mathrm{~cm}^{-1}$ peak in leaves, the area values were the sum of the areas obtained for the other bands, both for $C$. ladanifer and E. arborea $\left(4.97 \approx \sum(2.71+0.84+1.30) \quad\right.$ and $6.74 \approx \sum(4.75+0.79+1.44)$, respectively). This can be explained by the fact that this band is an indicator of the overall polysaccharide content (hemicellulose, lignin and cellulose).

On the basis of previous correlations, it is possible to differentiate the roots from the other plant components, which can be grouped under the term 'shoot'. The root areas $\left(A_{\text {root }}\right)$ ranged from 0.85 to 4.56 , while 
shoot areas $\left(A_{\text {shoot }}\right)$ were in the 3.41-15.50 range for both species (Table 4).

Root-to-shoot ratios based on vibrational data $\left(R_{\mathrm{FTIR}}\right)$ for $C$. ladanifer and E. arborea, for the four selected peaks, ranged from 0.25 to 0.33 and from 0.29 to 0.53 , respectively (see Table 4). In comparison with the results based on belowground and aerial biomass $\left(R_{\text {biomass }}=0.22\right.$ for $C$. ladanifer and $R_{\text {biomass }}=0.55$ for $E$. arborea), the closest values would be those associated with the peak at $1369 \mathrm{~cm}^{-1}$ (cellulose band) in the case of $C$. ladanifer $\left(R_{\mathrm{FTIR}}=0.25\right)$ and with the peak at $1514 \mathrm{~cm}^{-1}$ (lignin band) for $E$. arborea $\left(R_{\mathrm{FTIR}}=0.53\right)$. This is in agreement with the relative contents of vegetal components in the two shrubs under study: very high in cellulose for $C$. ladanifer and very high in lignin for $E$. arborea (Table 3 ).

\section{[TABLE 4]}

\section{Carbon and nitrogen concentrations}

Carbon concentrations were analysed for each the different components of the two shrubs (Table 5), omitting the one for $C$. ladanifer capsules due to its low representativeness and to allow comparison between the components of both species. The value of the carbon content (albeit slightly higher in the leaves) did not vary in a significant manner as regards the aerial and root parts, with values of $48.38 \pm 1.02 \%$ for $C$. ladanifer and $50.56 \pm 1.38 \%$ E. arborea. These values are in excellent agreement with those reported by Montero, Pasalodos-Tato, Montoto, et al. (2013) for the aerial part of the same species $\quad(49.70 \pm 0.66 \%$ and $51.43 \pm 1.17 \%$, respectively), to the Spanish Forest Map 1:25,000 (MFE25) values $(49.64 \pm 1.04 \%$ and $50.57 \pm 1.62 \%$, respectively) and are very close to the $50 \%$ value proposed by IPCC (2006).

\section{[TABLE 5]}

In relation to the amount of nitrogen per unit mass, it was maximum in the leaves for both species $\left(18.89 \mathrm{mg} \cdot \mathrm{g}^{-1}\right.$ and $10.46 \mathrm{mg} \cdot \mathrm{g}^{-1}$ for $C$. ladanifer and E. arborea, respectively).
The dissimilarity in the contents of $\mathrm{N}$ in the leaves between $\mathrm{Cl}$ and $\mathrm{Ea}$ (Table 5) can be explained according to equation 4 , provided that $S$ is substantially higher in $C$. ladanifer than in E. arborea and that $P$ is three times higher in April-May in species of genus Cistus (Cistus incanus) than in species of genus Erica (Erica multiflora) according to Catoni and Gratani (2014).

The distribution of nitrogen content, which resembles an inverse pyramid, reaching its maximum nitrogen content in the leaves and gradually decreasing as we move towards the root, where it presents its minimum, is consistent with that reported by García Rosa (2013) for fractions of $C$. ladanifer of different ages. Nonetheless, the value obtained in this study $(1.89 \%)$ is higher than that found by García Rosa (2013), around $0.91 \%$.

In the gum rockrose, the second highest nitrogen content -albeit substantially lowercorresponded to the thin branches $\left(8.42 \mathrm{mg} \cdot \mathrm{g}^{-}\right.$ $\left.{ }^{1}\right)$, followed by the roots $\left(3.62 \mathrm{mg} \cdot \mathrm{g}^{-1}\right)$ and by the thick branches $\left(2.66 \mathrm{mg} \cdot \mathrm{g}^{-1}\right)$. Conversely, in the briar root the leaves were followed by the thick branches $\left(3.78 \mathrm{mg} \cdot \mathrm{g}^{-1}\right)$ and by thin branches and roots (with almost identical values, 3.41 and $3.40 \mathrm{mg} \cdot \mathrm{g}^{-1}$, respectively). If the nitrogen content is analyzed considering the biomass of each fraction (Figure 6), it may be observed that the behavior is very different in the two species: the values for the analyzed fractions of $C$. ladanifer followed the order leaves $>$ thin branches $>$ thick branches $>$ roots, which was almost reversed to that in $E$. arborea: thick branches $>$ roots $>$ thin branches $>$ leaves.

[FIG. 6]

\section{Carbon stocks}

Upon calculation of the aboveground biomass for each of the species, according to Montero, Pasalodos-Tato, López-Senespleda, et al. (2013) (equations 5 and 6), the total amount of carbon stored (Table 6) was estimated with equation 7 , using the previously determined root-to-shoot ratios 
(Table 4) and the carbon concentrations in each of the fractions (Table 5). The carbon dioxide equivalent was obtained by direct conversion of the carbon stock using the ratio of their atomic weights $(44 / 12)$.

\section{[TABLE 6]}

In relation to the biomass (dry matter) values, the obtained value for $C$. ladanifer $\left(25.45 \mathrm{t} \mathrm{DW} \cdot \mathrm{ha}^{-1}\right)$ was significantly higher than those reported by Alías Gallego et al. (2009) (13.82 t DW $\mathrm{ha}^{-1}$ ) and García Rosa (2013) $\left(17 \mathrm{t} \cdot \mathrm{ha}^{-1}\right)$, but was similar to those reported by Basanta (1982) (27.26 t DW $\left.\cdot \mathrm{ha}^{-1}\right)$ or by Terradas (2001) (25 t DW $\left.\cdot \mathrm{ha}^{-1}\right)$.

It should be mentioned that, although the aboveground biomass value for E. arborea $\left(25.61 \mathrm{t} \mathrm{DW} \cdot \mathrm{ha}^{-1}\right)$ was only $19 \%$ higher than that reported by Navarro (2004) (21.39 t $\left.\mathrm{DW} \cdot \mathrm{ha}^{-1}\right)$, the total biomass value $(39.70 \mathrm{t}$ $\mathrm{DW} \cdot \mathrm{ha}^{-1}$ ) would be $85 \%$ higher than Navarro's estimation. Therefore, regardless of whether biomass multiplied by carbon concentration or carbon equations are directly used for carbon quantification, omitting the belowground biomass would be misleading, as stated by Koehler, Watzlawick, and Kirchner (2002), and it would seriously affecting the accuracy of carbon stock estimates.

The carbon stock values for $C$. ladanifer (10.07 tC $\cdot \mathrm{ha}^{-1}$ and $2.19 \mathrm{tC} \cdot \mathrm{ha}^{-1}$ for the shoots and roots, respectively) were -as expectedhigher than those reported by Alías et al. (2015), García Rosa (2013): $8.05 \mathrm{tC} \cdot \mathrm{ha}^{-1}$ and $1.41 \mathrm{tC} \cdot \mathrm{ha}^{-1}$, respectively. Nonetheless, these differences may be ascribed to the fact the latter are average values for specimens of very different ages (ranging from 0-2 year-old to 25-55 year-old specimens), whereas the specimens studied in this work were much more homogeneous (25-35 year-old specimens) and the associated biomass would be at its maximum (as noted by García Rosa (2013)).

It is also worth noting that the biomass values for both species were significantly higher than those of other shrubs: $c a .16 \mathrm{t}^{\mathrm{h}} \mathrm{ha}^{-}$ , $14 \mathrm{t} \cdot \mathrm{ha}^{-1}, 8.36 \mathrm{t} \cdot \mathrm{ha}^{-1}$, and $3.17 \mathrm{t} \cdot \mathrm{ha}^{-1}$ for
Asparagus albus, Genista sp., Rosmarinus officinalis and Retama sphaerocarpa, respectively (García Rosa 2013). In turn, the carbon sequestration associated to $C$. ladanifer and E.arborea shrublands would also be significantly higher.

\section{Conclusions}

In this work, different fractions of two shrub species present in significant volumes in Mediterranean areas, namely Cistus ladanifer and Erica arborea, have been studied by several techniques. A faster, cheaper and less time-consuming method for root-to-shoot ratio calculation based on vibrational data has been proposed: by using the areas under selected peaks in the infrared spectra, an excellent agreement with the results from UN-ECE/FAO-IPCC/Sanquetta et al. methodology ( $\left.R_{\text {biomass }}\right)$ was obtained, attaining the best correspondences for the peak at $1369 \mathrm{~cm}^{-1}$ (cellulose band) in the case of $C$. ladanifer $\left(R_{\mathrm{FTIR}}=0.25 ; R_{\text {biomass }}=0.22\right)$ and for the peak at $1514 \mathrm{~cm}^{-1}$ (lignin band) for $E$. arborea $\quad\left(R_{\mathrm{FTIR}}=0.53 ; \quad R_{\text {biomass }}=0.55\right)$. The elemental analysis confirmed that the percentage of carbon in the aerial and radical fractions did not differ in a significant manner, so the use of a 0.5 global value for the entire plant can be deemed as appropriate for both species. The percentage distribution of the biomass showed significant differences between the two species. As regards carbon storage, since carbon content did not depend on the analyzed fraction but was directly related to biomass, it could then be directly quantified from the aerial biomass (which is relatively easy to determine) using the rootto-shoot ratios. The carbon stock values (12.27 and $19.86 \mathrm{tC} \cdot \mathrm{ha}^{-1}$ for C. ladanifer and E. arborea, respectively) were substantially higher than those of other shrubs, evincing the importance of these two shrubs species for the mitigation of Climate Change and their suitability for ecological restoration purposes, in particular for poor soils. 
Acknowledgements

P.C.-P would like to thank Professor Z. Clérigo-Pérez for his support in the data sampling.

Funding

This work was supported by the European Union LIFE+ Programme under project $" \mathrm{CO}_{2}$ Operation: Integrated agroforestry practices and nature conservation against climate change", ref. LIFE11 ENV/ES/000535.

Conflict of interest

The authors declare no competing financial interests.

\section{References}

Agren, G. I., and T. Ingestad. 1987. "Root: shoot ratio as a balance between nitrogen productivity and photosynthesis." Plant, Cell and Environment 10 (7):579-586. doi: 10.1111/1365-3040.ep11604105.

Alías Gallego, J. C., M. García Rosa, C. Valares Masa, T. Sosa Díaz, and N. Chaves Lobón. 2009. "El matorral como sumidero de carbono." V Congreso Forestal Español, Ávila, Spain.

Alías, J. C., M. García, T. Sosa, C. Valares, and N. Chaves. 2015. "Carbon storage in the different compartments of two systems of shrubs of the southwestern Iberian Peninsula." Agroforestry Systems 89 (4):575-585 . doi: 10.1007/s10457-0159792-z.

Amir, S., M. Hafidi, L. Lemee, G. Merlina, M. Guiresse, E. Pinelli, J. C. Revel, J. R. Bailly, and A. Ambles. 2006. "Structural characterization of humic acids, extracted from sewage sludge during composting, by thermochemolysis-gas chromatography-mass spectrometry." Process Biochemistry 41 (2):410-422. doi: 10.1016/j.procbio.2005.07.005.

Baker, Matthew J., Júlio Trevisan, Paul Bassan, Rohit Bhargava, Holly J. Butler, Konrad M. Dorling, Peter R. Fielden, Simon W. Fogarty, Nigel J. Fullwood, Kelly a Heys, Caryn Hughes, Peter Lasch, Pierre L. Martin-Hirsch, Blessing Obinaju, Ganesh D. Sockalingum, Josep Sulé-Suso, Rebecca J. Strong, Michael J. Walsh, Bayden R. Wood, Peter Gardner, and Francis L. Martin. 2014. "Using Fourier transform IR spectroscopy to analyze biological materials." Nature protocols 9 (8):1771-91. doi: 10.1038/nprot.2014.110. Barboni, Toussaint, Grazia Pellizzaro, Bachisio Arca, Nathalie Chiaramonti, and Pierpaolo Duce. 2010. "Analysis and origins of volatile organic compounds smoke from ligno-cellulosic fuels." Journal of Analytical and Applied Pyrolysis $89 \quad$ (1):60-65. doi: 10.1016/j.jaap.2010.05.006.

Basanta, A. 1982. "Vegetación serial en Sierra Morena. Estudio ecológico de las respuestas del matorral a distintas intervenciones en el Coto Nacional "La Pata del Caballo" (Huelva)." PhD, Universidad de Sevilla.

Blanco Oyonarte, P., and R. M. Navarro Cerrillo. 2003. "Aboveground phytomass models for major species in shrub ecosystems of western Andalusia." Forest Systems 12 (3):47-55. doi: 10.5424/1078.

Castro-Díez, Pilar, Gabriel Montserrat-martí, and J. H. C. Cornelissen. 2003. "Trade-offs between phenology, relative growth rate, life form and seed mass among 22 Mediterranean woody species." Plant Ecology 166 (1):117-129. doi: 10.1023/a:1023209230303.

Castro, Isabel, Miguel Ángel Casado, Lucía Ramírez-Sanz, José Manuel de Miguel, Margarita Costa, and Francisco Díaz Pineda. 1996. "Funciones de estimación de la biomasa aérea de varias especies del matorral mediterráneo del centro de la península Ibérica." Orsis : Organismes $i$ Sistemes 11:107-116.

Catoni, Rosangela, and Loretta Gratani. 2014. "Variations in leaf respiration and photosynthesis ratio in response to air temperature and water availability among Mediterranean evergreen species." Journal of Arid Environments 102:82-88. doi: 10.1016/j.jaridenv.2013.11.013.

Cerrillo, R. M. Navarro, and P. Blanco Oyonarte. 2006. "Estimation of above-ground biomass in shrubland ecosystems of southern Spain." Investigación agraria. Sistemas y recursos forestales 15 (2):197207. doi: 10.5424/srf/2006152-00964.

Chen, Huilun, Carlo Ferrari, Marco Angiuli, Jun Yao, Costantino Raspi, and Emilia Bramanti. 2010. "Qualitative and quantitative analysis of wood samples by Fourier transform infrared spectroscopy and multivariate analysis." Carbohydrate 
Polymers $82 \quad$ (3):772-778. doi: 10.1016/j.carbpol.2010.05.052.

de Mello, Anabel, Leif Nutto, Karla Weber, Carlos Sanquetta, Jorge Monteiro de Matos, and Gero Becker. 2012. "Individual biomass and carbon equations for Mimosa scabrella Benth. (bracatinga) in southern Brazil." Silva Fennica 46 (3):333-343. doi: 10.14214/sf.44.

Emmanuel, Vartanian, Barres Odile, and Roque Céline. 2015. "FTIR spectroscopy of woods: A new approach to study the weathering of the carving face of a sculpture." Spectrochimica Acta - Part A: Molecular and Biomolecular Spectroscopy 136 (Part C):1255-1259. doi: 10.1016/j.saa.2014.10.011.

Evans, P. A. 1991. "Differentiating "hard" from "soft" woods using Fourier transform infrared and Fourier transform spectroscopy." Spectrochimica Acta Part A: Molecular Spectroscopy 47 (910):1441-1447. doi: 10.1016/05848539(91)80235-b.

Faust, Ben. 1997. Modern Chemical Techniques : An Essential Reference for Students and Teachers: Royal Society of Chemistry.

Federici, Sandro, Francesco N. Tubiello, Mirella Salvatore, Heather Jacobs, and Josef Schmidhuber. 2015. "New estimates of $\mathrm{CO} 2$ forest emissions and removals: 1990-2015." Forest Ecology and Management 352:89-98. doi: 10.1016/j.foreco.2015.04.022.

Ferro, Miguel D., Maria C. Fernandes, Ana F. C. Paulino, Sónia O. Prozil, Janis Gravitis, Dmitry V. Evtuguin, and Ana M. R. B. Xavier. 2015. "Bioethanol production from steam explosion pretreated and alkali extracted Cistus ladanifer (rockrose)." Biochemical Engineering Journal 104:98-105. doi: 10.1016/j.bej.2015.04.009.

García Rosa, María. 2013. "Estudio de la biomasa de Cistus ladanifer L. y Retama sphaerocarpa L. como sumidero de $\mathrm{CO}_{2}$ : existencias y potencialidad." Departamento de Biología Vegetal, Ecología y Ciencias de la Tierra, Universidad de Extremadura, Universidad de Extremadura. España (http://hdl.handle.net/10662/577).

Gayoso A, Jorge, and Javier Guerra C. 2005. "Contenido de carbono en la biomasa aérea de bosques nativos en Chile."
Bosque (Valdivia) 26 (2):33-38. doi: 10.4067/s0717-92002005000200005.

Griffiths, Peter R., and James A. De Haseth. 2007. Fourier Transform Infrared Spectrometry: John Wiley \& Sons.

Hilbert, D. W. 1990. "Optimization of Plant Root: Shoot Ratios and Internal Nitrogen Concentration." Annals of Botany 66 (1):91-99.

Huck, Christian W. 2015. "Advances of infrared spectroscopy in natural product research." Phytochemistry Letters 11:384-393. doi: 10.1016/j.phytol.2014.10.026.

Ibàñez, J. J., J. Vayreda, and C. Gracia. 2002. "Metodología complementaria al Inventario Forestal Nacional en Catalunya." In El Inventario Forestal Nacional. Elemento clave para la Gestión Forestal Sostenible, edited by F. Bravo, M. Del Río and C. Del Peso, 67-77. Fundación General de la Universidad de Valladolid.

IPCC. 2006. 2006 IPCC Guidelines for National Greenhouse Gas Inventories Agriculture, Forestry and Other Land Use. Edited by H.S. Eggelston, L. Buendia, K. Miwa, T. Ngara and K. Tanabe. Vol. 4, Guidelines for National Greenhouse Gas Inventories. Hayama, Japan: IPCC National Greenhouse Gas Inventories Programme - Institute for Global Environmental Strategies.

Kauppi, P. E., K. Mielikainen, and K. Kuusela. 1992. "Biomass and Carbon Budget of European Forests, 1971 to 1990." Science 256 (5053):70-74. doi: 10.1126/science.256.5053.70.

Kauppi, Pekka E., Erkki Tomppo, and Ari Ferm. 1995. "C and $\mathrm{N}$ storage in living trees within Finland since 1950s." Plant and Soil 168-169 (1):633-638. doi: 10.1007/bf00029377.

Koehler, E. S., L. F. Watzlawick, and F. F. Kirchner. 2002. "Fontes e níveis de erros nas estimativas do potencial de fixação de carbono." In As florestas e o carbono, edited by C. R. Sanquetta, L. F. Watzlawick, R. Balbinot, M. A. B. Ziliotto and F. S. Gomes, 251-264. Curitiba, Brasil: Imprensa Universitaria da UFPR.

Kollmann, Franz. 1959. Tecnología de la madera y sus aplicaciones. 2 vols. Madrid, Spain: Instituto Forestal de Investigaciones y 
Experiencias y Servicios de la Madera. Ministerio de Agricultura.

Leroy, Valrie, Eric Leoni, and Dominique Cancellieri. 2010. "Thermal Degradation of Lignocellulosic Fuels: Biopolymers Contribution." In Biopolymers, edited by Magdy Elnashar, 303-318. Rijeka, Croatia: InTech.

Lin, Y. J., C. P. Liu, and J. C. Lin. 2002. "Measurement of specific gravity and carbon content of important timber species in Taiwan." Taiwan Journal of Forest Science 17 (3):291-299.

Löwe, Helmut, Günther Seufert, and Frank Raes. 2000. "Comparison of methods used within Member States for estimating $\mathrm{CO}_{2}$ emissions and sinks according to UNFCCC and EU Monitoring Mechanism: forest and other wooded land." Biotechnologie, agronomie, société et environnement 4 (4):315-319.

Lloret, F., C. Casanovas, and J. Penuelas. 1999. "Seedling survival of Mediterranean shrubland species in relation to root:shoot ratio, seed size and water and nitrogen use." Functional Ecology 13 (2):210-216. doi: 10.1046/j.1365-2435.1999.00309.x.

MAPAMA. 2006. "Spanish Forest Map (MFE50)." Spanish Ministry of Agriculture, Food and Environment. http://www.mapama.gob.es/es/biodiversid ad/servicios/banco-datosnaturaleza/informaciondisponible/mfe50.aspx.

MAPAMA. 2016. "Spanish National Forest Inventory." Spanish Ministry of Agriculture, Food and Environment. http://www.mapama.gob.es/es/desarrollorural/temas/politica-forestal/inventariocartografia/inventario-forestal-nacional/.

Masalles, R. M., and J. Vigo. 1987. "La successió a les terres mediterrànies: sèries de vegetació." In Ecosistemes terrestres: la resposta als incendis $i$ a d'altres pertorbacions, edited by J. Terradas, 2743. Barcelona, Spain: Quaderns d'Ecologia Aplicada.

Mendoza-Ponce, Alma, and Leopoldo Galicia. 2010. "Aboveground and belowground biomass and carbon pools in highland temperate forest landscape in Central Mexico." Forestry 83 (5):497-506. doi: 10.1093/forestry/cpq032.

Mokany, Karel, R. John Raison, and Anatoly S. Prokushkin. 2006. "Critical analysis of root:shoot ratios in terrestrial biomes." Global Change Biology 12 (1):84-96. doi: 10.1111/j.1365-2486.2005.001043.x.

Montero, G, M Pasalodos-Tato, E LópezSenespleda, R Onrubia, and G Madrigal. 2013. "Ecuaciones para la estimación de la biomasa en matorrales y arbustedos mediterráneos." $6^{\circ}$ Congreso Forestal Español, Vitoria-Gasteiz, Spain, June 1014, 2013.

Montero, G., M Pasalodos-Tato, R. Montoto, E. Lopez-Senespleda, R. Onrubia, A. BravoOviedo, and R Ruiz-Peinado. 2013. "Contenido de Carbono en la biomasa de las principales especies de matorral y arbustedos de España." $6^{\circ}$ Congreso Forestal Español, Vitoria-Gasteiz, Spain, June 10-14, 2013.

Montero, Gregorio, Ricardo Ruiz-Peinado, and Marta Muñoz. 2005. Producción de biomasa y fijación de $\mathrm{CO}_{2}$ por los bosques españoles. Vol. 13, Monografías INIA: Serie Forestal. Madrid, Spain: Instituto Nacional de Investigación $\mathrm{y}$ Tecnología Agraria y Alimentaría (INIA).

Navarro, Rafael M. 2004. "Fitomasa aérea en los ecosistemas de matorral en el monte Can Vilallonga (T.M. DE Cassà de la SelvaGirona)." Ecología 18:99-112.

Orellana-Rivadeneyra, G., M.L. Sandoval-Saolís, G. Linares-Fleites, N.E. García Calderón, and J.V.; Tamariz-Flores. 2012. "Description of the carbon dynamics in forest soils using a model of reservoirs." Avances en Ciencias e Ingeniería 3 (1):123-135.

Owen, N. L., and D. W. Thomas. 1989. "Infrared studies of "hard" and "soft" woods." Applied Spectroscopy 43 (3):451-455. doi: 10.1366/0003702894202760.

Paton, D., R. Osorio, P. Azocar, H. Bote, A. Matas, and J. Tovar. 1997. "Prediction of browsing biomass of certain shrub species from arid Mediterranean climate of North Chile by multicriteria analysis." Archivos de zootecnia 46 (175):225-237.

Ruiz-Peinado, R., G. Montero, and M. Del Rio. 2012. "Biomass models to estimate carbon stocks for hardwood tree species." Forest Systems 21 (1):42-52. doi: 10.5424/fs/2112211-02193.

Ruiz-Peinado, Ricardo, Miren del Rio, and Gregorio Montero. 2011. "New models for estimating the carbon sink capacity of 
Spanish softwood species." Forest Systems 20 (1):176-188.

Sanquetta, C.R., A.P.D. Corte, R. Balbinot, and M.A.B. Zilliotto. 2004. "Proposta metodológica para quantificação e monitoramento do carbono estocado em florestas plantadas." In Mercado de carbono: mercado e ciência, edited by C.R. Sanquetta and M.A.B. Zilliotto, 240255. Curitiba, Brazil: UFPR / ECOPLAN.

Sanquetta, Carlos R., Ana P. D. Corte, and Fernando da Silva. 2011. "Biomass expansion factor and root-to-shoot ratio for Pinus in Brazil." Carbon Balance and Management 6 (6):1-8. doi: 10.1186/1750-0680-6-6.

Schenk, H. Jochen, and Robert B. Jackson. 2002. "Rooting depths, lateral root spreads and below-ground/above-ground allometries of plants in water-limited ecosystems." Journal of Ecology 90 (3):480-494. doi: 10.1046/j.1365-2745.2002.00682.x.

Schwanninger, M., J. C. Rodrigues, H. Pereira, and B. Hinterstoisser. 2004. "Effects of short-time vibratory ball milling on the shape of FT-IR spectra of wood and cellulose." Vibrational Spectroscopy 36 (1):23-40. doi: 10.1016/j.vibspec.2004.02.003.

Serrada, R., G. Montero, and J.A. Reque Kilchenmann. 2008. Compendium of applied forestry in Spain. Madrid, Spain: Instituto Nacional de Investigación y Tecnología Agraria y Alimentaria (INIA).

Somogyi, Z, E Cienciala, R Mäkipää, $\mathrm{P}$ Muukkonen, A Lehtonen, and P Weiss. 2007. "Indirect methods of large-scale forest biomass estimation." European Journal of Forest Research 126 (2):197207. doi: 10.1007/s10342-006-0125-7.

Spanish Biodiversity Foundation, and Spanish Royal Botanic Garden. 2011. "Anthos. Spanish plants information system." Spanish Ministry of Agriculture, Food and Environment and Spanish State Agency for National Research Council. http://www.anthos.es/index.php?lang=en.

Terradas, J. 2001. Ecología de la vegetación: de la ecofisiología de las plantas a la dinámica de comunidades y paisajes. Barcelona, Spain: Omega.

Traoré, Mohamed, Joeri Kaal, and Antonio Martínez Cortizas. 2016. "Application of FTIR spectroscopy to the characterization of archeological wood." Spectrochimica
Acta - Part A: Molecular and Biomolecular Spectroscopy 153:63-70. doi: 10.1016/j.saa.2015.07.108.

UN-ECE/FAO. 2000. Forest resources of Europe, CIS, North America, Australia, Japan, and New Zealand (industrialized temperate/boreal countries) : UNECE/FAO contribution to the Global Forest Resources Assessment 2000. Vol. 17, Geneva timber and forest study papers. New York, USA: United Nations, Economic Commission for Europe, Timber Section; Food and Agriculture Organization of the United Nations.

Wang, Jinchuang, Baoli Duan, and Yuanbin Zhang. 2011. "Effects of experimental warming on growth, biomass allocation, and needle chemistry of Abies faxoniana in even-aged monospecific stands." Plant Ecology $213 \quad$ (1):47-55. doi: 10.1007/s11258-011-0005-1.

Watson, Robert T., and Ian R. Noble. 2002. "Carbon and the Science-Policy Nexus: The Kyoto Challenge." In Challenges of a Changing Earth, edited by Will Steffen, Jill Jäger, David J. Carson and Clare Bradshaw, 57-64. Berlin, Heidelberg: Springer Berlin Heidelberg. 


\section{Tables}

Table 1. Main absorption bands in the FTIR spectra of the different fractions of C. ladanifer and E. arborea and their assignments according to the literature (Emmanuel, Odile, and Céline 2015, Faust 1997, Schwanninger et al. 2004, Traoré, Kaal, and Martínez Cortizas 2016).

\begin{tabular}{|c|c|c|c|c|c|c|c|c|c|c|}
\hline \multicolumn{8}{|c|}{ Wavenumber $\left(\mathrm{cm}^{-1}\right)$} & \multirow{2}{*}{ Vegetal component } & \multirow{2}{*}{ Bonds } & \multirow{2}{*}{ Assignment } \\
\hline $\mathrm{L} \mathrm{Cl}$ & $\mathrm{t} C l$ & $\mathrm{~T} \mathrm{Cl}$ & $\mathrm{RCl}$ & $\mathrm{L} E a$ & $\mathrm{t} E a$ & T Ea & $\mathrm{R} E a$ & & & \\
\hline 1035 & 1050 & 1035 & 1035 & 1035 & 1050 & 1039 & 1031 & Cellulose, hemicellulose, lignin & $\begin{array}{l}\mathrm{C}-\mathrm{O}, \mathrm{C}=\mathrm{C} \\
\text { and } \mathrm{C}-\mathrm{C}-\mathrm{O}\end{array}$ & $\begin{array}{l}\text { Aromatic } \mathrm{C}-\mathrm{H} \text { in plane deformation; plus } \mathrm{C}-\mathrm{O} \text { deformation in primary } \\
\text { alcohols; plus } \mathrm{C}=\mathrm{O} \text { stretch (unconjugated) }\end{array}$ \\
\hline & & & & & & & 1081 & Cellulose & $\mathrm{C}-\mathrm{H}$ & $\mathrm{C}-\mathrm{O}$ deformation in secondary alcohols and aliphatic ethers \\
\hline 1164 & & & & & & & 1155 & Cellulose & $\mathrm{C}-\mathrm{H}$ & $\mathrm{C}-\mathrm{O}-\mathrm{C}$ asymmetric valence vibration, $\mathrm{C}=\mathrm{O}$ stretching in aliphatic groups \\
\hline 1233 & 1243 & 1243 & 1251 & 1243 & 1247 & 1247 & 1247 & Lignin & $\mathrm{C}-\mathrm{H}$ & $\mathrm{C}-\mathrm{C}$ plus $\mathrm{C}-\mathrm{O}$ plus $\mathrm{C}=\mathrm{O}$ stretching $(\mathrm{OH}$ plane deformation, also $\mathrm{COOH})$ \\
\hline 1317 & 1320 & 1320 & 1321 & & 1331 & 1332 & & Cellulose, hemicellulose, lignin & $\mathrm{C}-\mathrm{O}, \mathrm{CH}_{2}$ & $\begin{array}{l}\text { Condensation of guaiacyl unit and syringyl unit, syringyl unit and } \mathrm{CH}_{2} \\
\text { bending stretching; } \mathrm{CH}_{2} \text { rocking vibration }\end{array}$ \\
\hline 1367 & 1375 & 1375 & 1375 & 1373 & 1375 & 1375 & 1374 & Cellulose & $\mathrm{C}-\mathrm{H}$ & In-plane bending vibration of the $\mathrm{C}-\mathrm{H}$ and $\mathrm{C}-\mathrm{O}$ groups of the hexose ring \\
\hline 1455 & 1458 & 1457 & 1456 & 1455 & 1464 & 1459 & 1452 & Saccharide backbone & $\mathrm{C}-\mathrm{H}$ & $\begin{array}{l}\text { Alkane deformation relating to } \mathrm{CH} \text { and } \mathrm{CH}_{2} \text {, consistent with the saccharide } \\
\text { backbone }\end{array}$ \\
\hline 1515 & 1508 & 1508 & 1509 & 1517 & 1508 & 1513 & 1513 & Lignin & $\mathrm{C}=\mathrm{C}$ & Stretching vibration in the aromatic skeletal vibration \\
\hline 1615 & 1618 & 1617 & 1617 & 1616 & 1617 & 1617 & 1618 & Cellulose, lignin & $\mathrm{O}-\mathrm{H}, \mathrm{C}-\mathrm{O}$ & Absorbed $\mathrm{O}-\mathrm{H}$ and conjugated $\mathrm{C}-\mathrm{O}$ lignin or cellulose \\
\hline 1733 & 1735 & 1735 & 1736 & 1733 & 1739 & 1740 & 1736 & Hemicellulose + lignin & $\mathrm{C}=\mathrm{O}$ & Ester linkage of the carboxylic group of ferulic and p-coumaric acids \\
\hline 2850 & & & & 2852 & & & & Cellulose, hemicellulose, lignin & $\mathrm{CH}$ stretch & Symmetric $\mathrm{CH}_{2}$ valence vibration \\
\hline 2919 & 2921 & 2920 & 2919 & 2923 & 2918 & 2919 & 2928 & Cellulose, hemicellulose, lignin & $\mathrm{CH}$ stretch & \\
\hline 3389 & 3413 & 3421 & 3405 & 3374 & 3409 & 3409 & 3405 & Lignin & $\mathrm{O}-\mathrm{H}$ & Intramolecular $\mathrm{O}_{3}-\mathrm{H} \cdots \mathrm{O}_{5}{ }^{\prime}$ in cellulose \\
\hline
\end{tabular}

Cl: Cistus ladanifer; Ea: Erica arborea; L: leaves; t: thin branches; T: thick branches; R: roots. 
Table 2. Corrected areas values for each fraction in C. ladanifer and E. arborea.

\begin{tabular}{|c|c|c|c|c|c|c|c|c|}
\hline \multirow{2}{*}{$\begin{array}{c}\text { Wavenumber } \\
\left(\mathrm{cm}^{-1}\right)\end{array}$} & \multicolumn{4}{|c|}{ Cistus ladanifer $\mathbf{L}$. } & \multicolumn{4}{|c|}{ Erica arborea L. } \\
\hline & Leaves & Thin branches & Thick branches & Roots & Leaves & Thin branches & Thick branches & Roots \\
\hline 1730 & 2.71 & 6.22 & 3.90 & 4.21 & 4.75 & 5.98 & 4.77 & 4.56 \\
\hline 1514 & 0.84 & 1.30 & 0.86 & 0.85 & 0.79 & 1.20 & 1.75 & 1.97 \\
\hline 1458 & 4.97 & 3.97 & 2.66 & 2.87 & 6.74 & 3.04 & 2.60 & 3.55 \\
\hline 1369 & 1.30 & 1.26 & 0.93 & 0.88 & 1.44 & 1.03 & 0.94 & 1.15 \\
\hline
\end{tabular}

Table 3. Percentages in terms of vegetal components for C. ladanifer and E. arborea (Ferro et al. 2015, Barboni et al. 2010, Leroy, Leoni, and Cancellieri 2010).

\begin{tabular}{ccc}
\hline & Cistus ladanifer & Erica arborea \\
\hline Cellulose (\%) & $54.9-55.7$ & $37.3-41.1$ \\
Lignin (\%) & $24.5-34.2$ & $39.3-40.1$ \\
Hemi-cellulose (\%) & $10.1-10.9$ & $9.7-13.8$ \\
Extractive (\%) & $9.4-9.6$ & $5.7-11.0$ \\
\hline
\end{tabular}

Table 4. Comparison of root-to-shoot ratios for $C$. ladanifer and E. arborea determined by two methodologies: using vibrational data $\left(R_{\mathrm{FTIR}}\right)$ and using the UN-ECE/FAO-IPCC procedure modified by Sanquetta, Corte, and da Silva (2011) ( $\left.R_{\text {biomass }}\right)$.

\begin{tabular}{|c|c|c|c|c|c|c|c|c|}
\hline & \multicolumn{4}{|c|}{ C. ladanifer } & \multicolumn{4}{|c|}{ E. arborea } \\
\hline \multicolumn{9}{|l|}{ FTIR method } \\
\hline Wavenumber $\left(\mathrm{cm}^{-1}\right)$ & 1730 & 1514 & 1458 & 1369 & 1730 & 1514 & 1458 & 1369 \\
\hline Root area & 4.21 & 0.85 & 2.87 & 0.88 & 4.56 & 1.97 & 3.55 & 1.15 \\
\hline Shoot area & 12.83 & 3.00 & 11.60 & 3.49 & 15.50 & 3.74 & $12-38$ & $3-41$ \\
\hline$R_{\mathrm{FTIR}}$ & 0.33 & 0.28 & 0.25 & 0.25 & 0.29 & 0.53 & 0.29 & 0.34 \\
\hline \multicolumn{9}{|c|}{$U N-E C E / F A O-I P C C$ modified by Sanquetta } \\
\hline$R_{\text {biomass }}$ & \multicolumn{4}{|c|}{0.22} & \multicolumn{4}{|c|}{0.55} \\
\hline
\end{tabular}

The underlined $R_{\text {FTIR }}$ values are the closest to those obtained by UN-ECE/FAO-IPCC method, modified by Sanquetta.

Table 5. C content, $\mathrm{N}$ content and $\mathrm{C}: \mathrm{N}$ ratios for $C$. ladanifer and E. arborea.

\begin{tabular}{|c|c|c|c|c|c|c|c|c|}
\hline & \multicolumn{4}{|c|}{ Cistus ladanifer $L}$. & \multicolumn{4}{|c|}{ Erica arborea $L}$. \\
\hline & Leaves & Thin branches & Thick branches & Roots & Leaves & Thin branches & Thick branches & Roots \\
\hline $\mathrm{C}$ content $\left(\mathrm{mg} \cdot \mathrm{g}^{-1}\right)$ & $\begin{array}{l}500.72 \\
(0.37)\end{array}$ & $\begin{array}{c}481.20 \\
(0.27)\end{array}$ & $\begin{array}{c}475.60 \\
(0.59)\end{array}$ & $\begin{array}{l}477.76 \\
(0.47)\end{array}$ & $\begin{array}{l}528.20 \\
(0.22)\end{array}$ & $\begin{array}{c}493.38 \\
(0.13)\end{array}$ & $\begin{array}{c}502.62 \\
(0.31)\end{array}$ & $\begin{array}{l}498.24 \\
(1.23)\end{array}$ \\
\hline $\mathrm{N}$ content $\left(\mathrm{mg} \cdot \mathrm{g}^{-1}\right)$ & $\begin{array}{l}18.89 \\
(0.02)\end{array}$ & $\begin{array}{c}8.42 \\
(0.00)\end{array}$ & $\begin{array}{l}2.66 \\
(0.18)\end{array}$ & $\begin{array}{c}3.62 \\
(0.02)\end{array}$ & $\begin{array}{l}10.46 \\
(0.03)\end{array}$ & $\begin{array}{c}3.41 \\
(0.18)\end{array}$ & $\begin{array}{l}3.78 \\
(0.04)\end{array}$ & $\begin{array}{c}3.40 \\
(0.16)\end{array}$ \\
\hline $\mathrm{C}: \mathrm{N}$ ratio & $\begin{array}{l}26.50 \\
(0.05)\end{array}$ & & & & $\begin{array}{l}50.52 \\
(0.14)\end{array}$ & & & \\
\hline
\end{tabular}

All values are given in average \pm standard deviations (in brackets).

Table 6. Estimated biomass (dry matter), carbon stock and carbon dioxide equivalent.

\begin{tabular}{cccccccccc}
\hline & \multicolumn{3}{c}{ Biomass $\left(\mathrm{t} \mathrm{DW} \cdot \mathrm{ha}^{-1}\right)$} & \multicolumn{3}{c}{ Carbon stock $\left(\mathrm{tC} \cdot \mathrm{ha}^{-1}\right)$} & \multicolumn{3}{c}{$\mathbf{C O}_{\mathbf{2}} \mathbf{e q}\left(\mathrm{t} \mathrm{CO} \mathrm{CO}_{2} \mathrm{eq}^{-1}\right)$} \\
\cline { 2 - 10 } & Shoot & Root & Total & Shoot & Root & Total & Shoot & Root & Total \\
\hline C. ladafiner & 20.86 & 4.59 & 25.45 & 10.07 & 2.19 & 12.27 & 36.94 & 8.04 & 44.97 \\
E. arborea & 25.61 & 14.09 & 39.70 & 12.84 & 7.02 & 19.86 & 47.07 & 25.73 & 72.80 \\
\hline
\end{tabular}




\section{Figure captions}
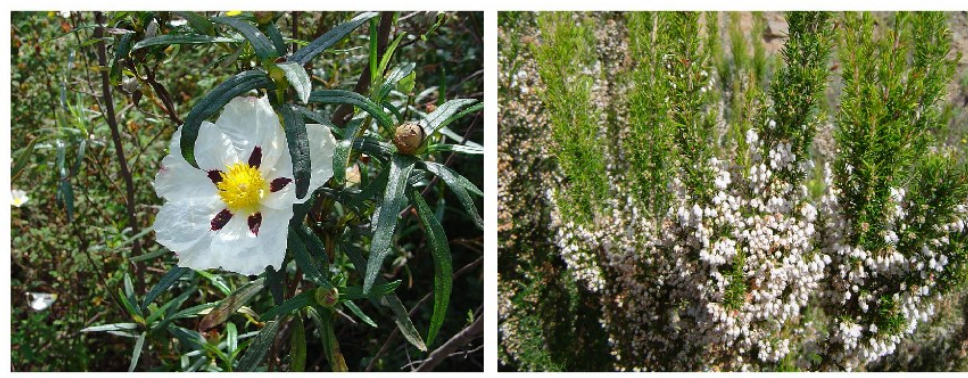

Figure 1. Cistus ladanifer L., also known as gum rockrose or labdanum (left); Erica arborea L., also known as briar root or tree heath (right).
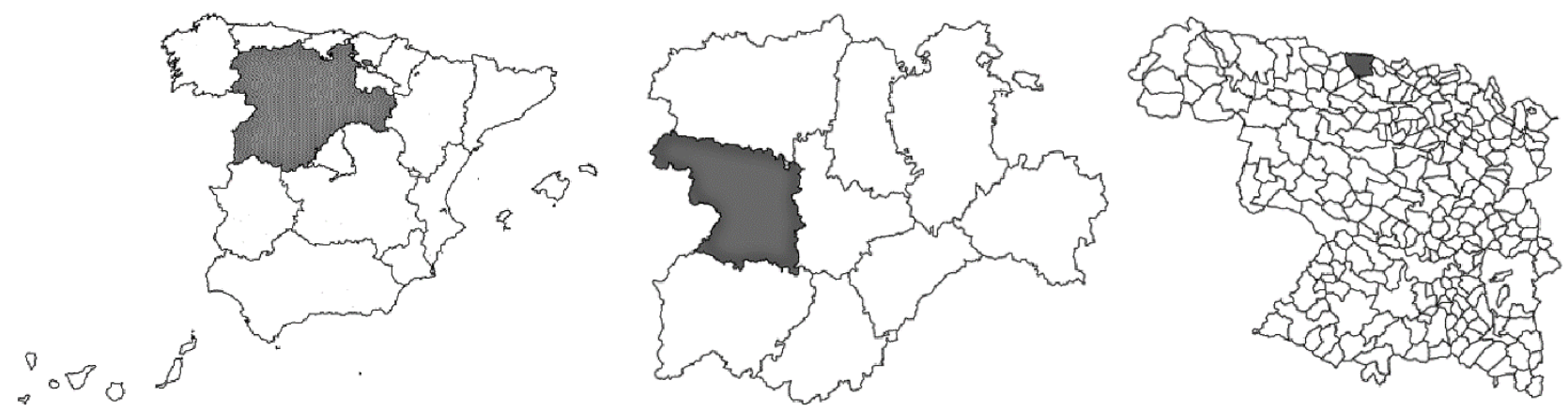

Figure 2. Location of Castilla y León region in the Iberian Peninsula (left); location of Zamora province in Castilla y León region (center); location of the shrubland under study in Zamora province (right).

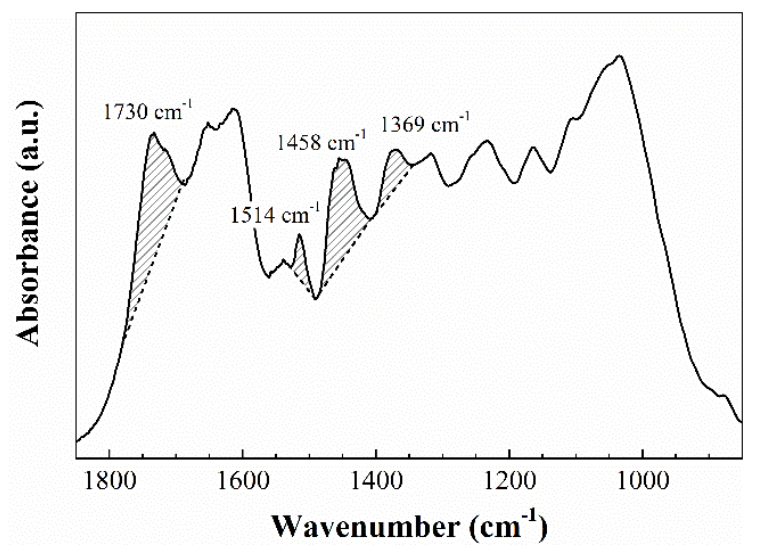

Figure 3. FTIR spectrum of E. arborea leaves with the corrected peak areas calculated at some selected wavenumbers $\left(\mathrm{cm}^{-1}\right)$. 


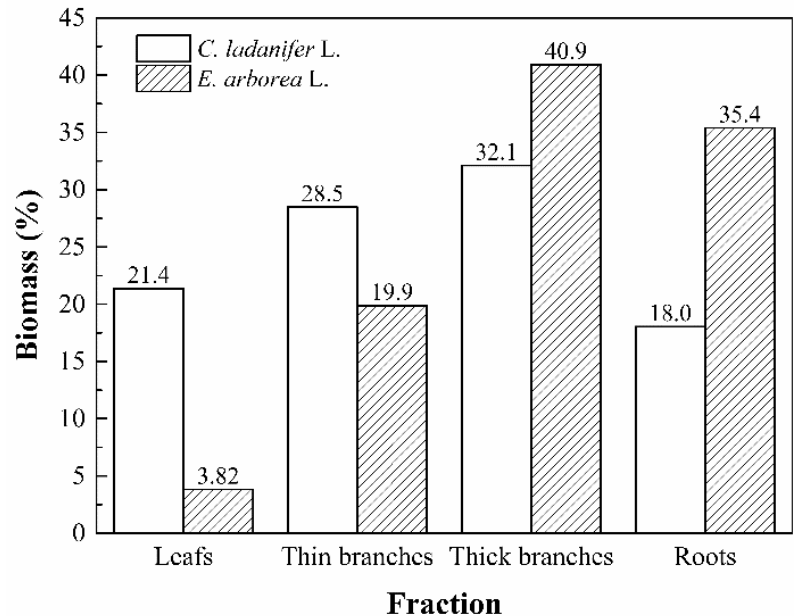

Figure 4. Biomass distribution for Cistus ladanifer L. and Erica arborea L.
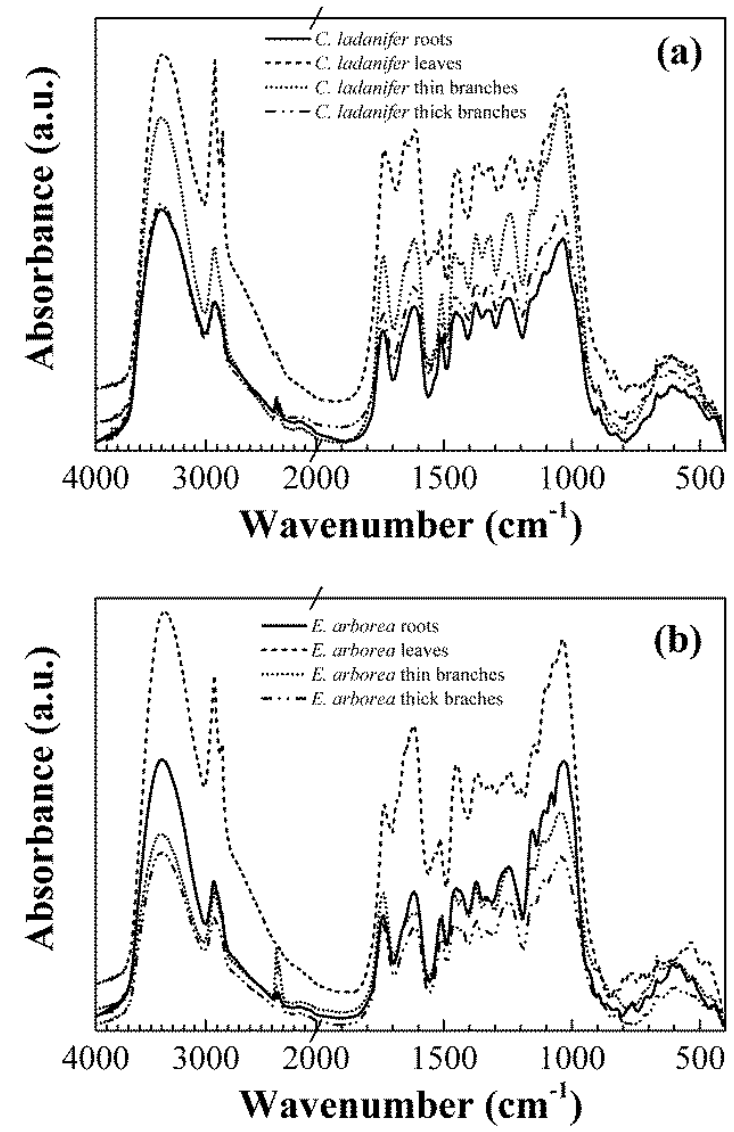

Figure 5. FTIR spectra of the different fractions (leaves, thin branches, thick branches and roots) of ( $a$ ) $C$. ladanifer and (b) E. arborea 


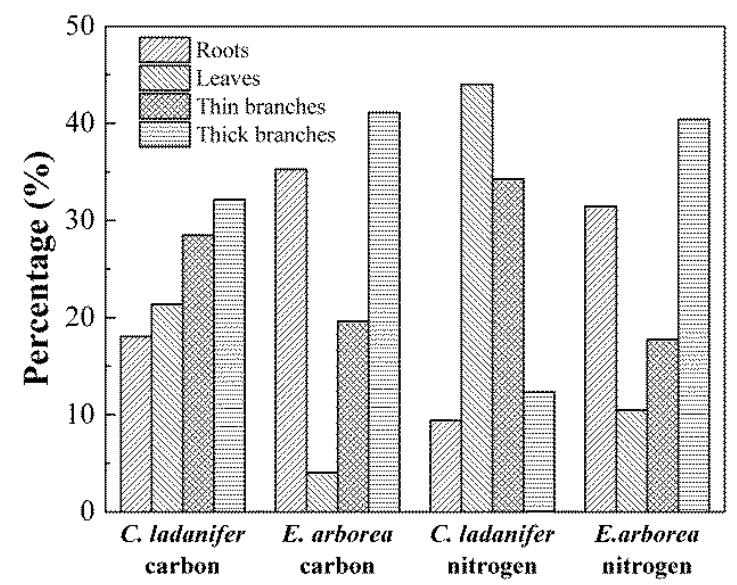

Figure 6. Contribution of the different components to the total carbon and nitrogen stocks, for C. ladanifer and E. arborea, taking into consideration the biomass distribution. 
5.2. Publicación \#2:

\section{VALORIZATION OF CISTUS LADANIFER AND ERICA ARBOREA SHRUBS FOR FUEL: WOOD AND BARK THERMAL CHARACTERIZATION (ENVIADA A EUROPEAN JOURNAL OF WOOD AND WOOD PRODUCTS)}

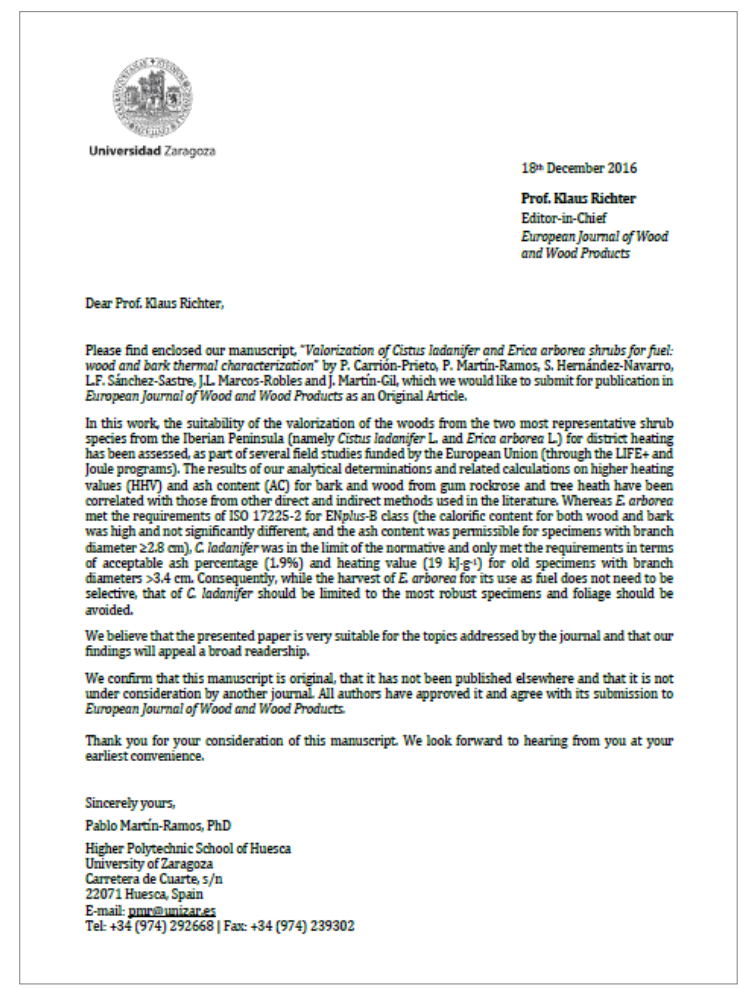





\title{
Valorization of Cistus ladanifer and Erica arborea shrubs for fuel: wood and bark thermal characterization
}

\author{
Paula Carrión-Prieto ${ }^{1}$, Pablo Martín-Ramos ${ }^{2 *}$, Salvador Hernández-Navarro ${ }^{1}$, Luis F. Sánchez- \\ Sastre $^{1}$, José L. Marcos-Robles ${ }^{1}$ and Jesús Martín-Gil ${ }^{1}$ \\ ${ }^{1}$ Agriculture and Forestry Engineering Department, ETSIIAA, Universidad de Valladolid. Avenida \\ de Madrid, 44, 34004 Palencia, Spain. \\ ${ }^{2}$ Department of Agricultural and Environmental Sciences, EPSH, Universidad de Zaragoza, \\ Carretera de Cuarte s/n, 22071 Huesca, Spain. Phone: +34 (974) 292668; Fax: +34 (974) 239302; \\ E-mail:pmr@unizar.es
}

\begin{abstract}
As a form of upgraded biomass characterized by its high energy density, low production costs, and low process energy requirements, wood pellets are an environmentally friendly fuel allowing for carbon neutral heating with high energy efficiency. In this work, the suitability of a valorization of the woods from the two most representative shrub species from the Iberian Peninsula (namely Cistus ladanifer L. and Erica arborea L.) for heating has been assessed. Whereas Erica arborea met the requirements of ISO 17225-2:2014 for ENplus-B class (the calorific content for both wood and bark was high and not significantly different, and the ash content was permissible for specimens with branch diameter $\geq 2.8 \mathrm{~cm}$ ), Cistus ladanifer was in the limit of the normative and only met the requirements in terms of acceptable ash percentage $(1.9 \%)$ and heating value $\left(19 \mathrm{~kJ} \cdot \mathrm{g}^{-1}\right)$ for old specimens with branch diameters $>3.4 \mathrm{~cm}$. Consequently, while the harvest of $E$. arborea for its use as fuel does not need to be selective, that of $C$. ladanifer should be limited to the most robust specimens and foliage should be avoided.
\end{abstract}

Keywords: ash content; biomass resources; gum rockrose; heating values; tree heath.

\section{Introduction}

A significant proportion of Mediterranean forest vegetation consists of evergreen small diameter hardwood shrubs, such as Cistus ladanifer L. (gum rockrose) and Erica arborea L. (tree heath), which have been traditionally used as fuelwood for domestic heating purposes. In the geographic area under study (Castilla y León, Spain) both species are so abundant that their utilization as a biomass resource for energy purposes has aroused significant interest ${ }^{2}$.

Of the two bushes into consideration, the most potentially profitable would be $E$. arborea, whose heating value was recently reported to be the highest of all evergreen Mediterranean hardwood species (Barboutis and Lykidis 2014).

\footnotetext{
${ }^{2}$ In fact, since 2012, field studies aimed at this valorization, funded by the European Union through the LIFE+ and Joule programs, have been conducted in several municipalities in the province of Zamora (Spain). The ultimate goal would be to collect tree heath and gum rockrose for their combustion in district heating facilities (municipal boilers) in Fabero (Soria, Spain) and Las Navas del Marqués (Ávila, Spain), as well as for the electricity production plant located in Garray (Soria, Spain).
} 
The use of biomass as an energy source provides substantial socio-economic and environmental benefits. However, bio-fuels have low bulk densities which limit their use to areas around their origin, being this drawback a restrictive factor for their energy use. Nevertheless, densification by pelleting minimizes this disadvantage. Global pellet production has considerably increased for the last years (from 7 to 19 million tons between 2006 and 2012 (Duca et al. 2014), mainly in Europe and North America, and the growth in pellet consumption has resulted in more diversity. Consequently, the industry has started looking for products, such as wastes obtained from forestry and scrubland wood. The doubtful quality of these materials originated the development of quality standards in some countries, so as to guarantee the right use of the different types of pellets in combustion equipment.

Due to differences in chemical structure, bark and wood from C. ladanifer and E. arborea should show different properties, and in particular, those related to their applicability as fuels. This differentiation is important because the bark of all evergreen hardwood species usually presents significantly higher ash content than wood and, in agreement with the international standard ISO 17225-2:2014 (ISO 2014) -which has recently superseded the European Standard, EN 14961-2, for the quality characteristics of pellets (European Pellet Council 2011)-, the threshold ash content value is $2 \%$. In this normative, the required net calorific value (NCV) or lower calorific value (LHV) is $\geq 16.56 \mathrm{~kJ} \cdot \mathrm{g}^{-1}$ and the higher heating value (HHV) is $\geq 18.82 \mathrm{~kJ} \cdot \mathrm{g}^{-1}$.

The aims of the study presented herein have been: $(i)$ to correlate the results of our analytical determinations and related calculations on HHV and ash content (AC) for bark and wood from $C$. ladanifer and E. arborea with those from other direct and indirect methods used in the literature; and (ii) to explore which bark diameters would meet the ISO 17225-2:2014 (ISO 2014)/ENplus (ENplus 2015) requirements for HHV and AC with a view to the valorization of these two shrub species as fuels. This is in line with the work by other authors on woods from other species (Duca et al. 2014, Miranda et al. 2017).

\section{Material and methods}

The quantity known as higher heating value (HHV) (also referred to as gross energy, upper heating value, gross calorific value (GCV) or higher calorific value (HCV)) is determined by bringing all the products of combustion back to the original pre-combustion temperature and, in particular, condensing any vapor produced. This is the same as the thermodynamic heat of combustion, since the enthalpy change for the reaction assumes a common temperature of the compounds before and after combustion, in which case the water produced by combustion is condensed to a liquid, hence yielding its latent heat of vaporization.

Calculations for the estimation of biomass and heating values may be obtained either by direct or by indirect methods. Direct methods involve the destruction of heavy biomass, whereas in indirect methods equations are used to estimate heating values from measurements of other variables, making the process easier (Bombelli et al. 2009).

In the first part of this study, heating values were determined by a destructive method, which comprised the selection, felling and extraction of biomass of each of the species and its subsequent combustion.

The aerial part was separated from the roots using a saw and then, following an analogous procedure to that described by Ruiz-Peinado Gertrudix et al. (2012), root systems were excavated by using a tractor with a shovel and then spades to complete the job. For each plant, soil was excavated down in a circular area of twice the mean crown diameter. In addition to the main body of the roots, those remaining in the hole were also collected. Samples were transported to the 
laboratory (ETSIIAA facilities, Universidad de Valladolid, Spain), where they were separated into different fractions and weighed (fresh weight). In the case of $C$. ladanifer, they were classified into leaves, xerochastic capsules, branches (thin: 3-7 mm in diameter; thick: 7-17 mm in diameter) and roots. On the other hand, for E. arborea -given its morphology and the impracticality of leaves separation- they were divided into four fractions: leaves with flowers and fruits, fine material $(<1$ $\mathrm{cm})$, thick material $(<5 \mathrm{~cm})$ and roots, in agreement with Mello et al. (2012).

In addition to aforementioned information, the stem diameter $(2 R)$, bark thickness $(f)$ and wood and bark percentages were characterized for both species. The proportion of bark was calculated as the ratio of bark area in a transverse section to the total stem area of this section, according to equation (Barmpoutis et al. 2015):

$$
Z=100\left[f \frac{(2 R-f)}{R^{2}}\right]
$$

where $Z=$ bark percentage $(\%), R=$ barked stem radius $(\mathrm{cm})$ and $f=$ bark thickness $(\mathrm{cm})$.

For the determination of the bark percentage, the transverse surfaces were assumed to be circular. Consequently, bark and wood were separated and the materials were ground by means of a portable chipper. The resulting data is summarized in Table1.

Table 1. Stem diameter and bark thickness of the shrub species under study. Values are given as an average of 10 repetitions, followed by the the minimum and maximum values in brackets.

\begin{tabular}{ccccc}
\hline Species & Stem diameter, $2 R(\mathrm{~cm})$ & Bark thickness, $f(\mathrm{~cm})$ & Bark, $Z(\%)$ & Wood (\%) \\
\hline C. ladanifer $\mathrm{L}$. & $1.9(1.8-2.3)$ & $0.15(0.07-0.20)$ & 29 & 71 \\
C. ladanifer $\mathrm{L}$. & $3.4(2.3-4.2)$ & $0.20(0.11-0.40)$ & 22.5 & 77.5 \\
(old specimens) & $2.8(2.6-3.6)$ & $0.18(0.10-0.30)$ & 25 & 75 \\
E. arborea $\mathrm{L}$. & & & \\
\hline
\end{tabular}

It should be noted that for the study of $C$. ladanifer two sets of individuals were selected: ones with average stem diameter (1.9 cm trunk diameter) and also robust old specimens (older than 12 years, according to the equation $y=1.5496 x+1.5342 R^{2}$ (Valares Masa et al. 2016)), with diameters above the average, provided that this second group was more likely to meet the EN standard. Ten repetitions were carried out for each group.

Calorific values, expressed as $\mathrm{HHV}$, for $C$. ladanifer and $E$. arborea fractions were calculated from elemental analysis data in agreement with the US Institute of Gas Technology (IGT) (Talwalkar et al. 1981): $H H V=0.341(\% C)+1.322(\% H)-0.12(\% O+\% N)$, where $\% \mathrm{C}, \% \mathrm{H}$, etc. are the mass fractions in $\mathrm{wt} \%$ of dry material and $\mathrm{HHV}$ the heating value for dry material in $\mathrm{MJ} / \mathrm{kg}$. Although originally derived from data on coal, this formula has been shown to give acceptable results for a wide range of carbonaceous materials including biomass (CHPQA 2008).

Alternatively, HHV values were also calculated from holocellulose and lignine+extractives percentages, following the guidelines of Aseeva et al. (2005) and Kienzle et al. (2001) and applying a factor of 17.5 for holocellulose and of 25.5 for the lignine+extractives mixture.

Experimental HHV values were determined in a Parr 1261 isoperibol bomb calorimeter (Thermo Fisher Scientific, Waltham, MA, USA) according to the method described in BS EN 14918:2009 standard (BSI 2010). Other experimental values, such as the total enthalpy of combustion, were obtained from differential scanning calorimetry (DSC) curves by numerical integration of the experimental signal on the whole temperature range $\left(30-600{ }^{\circ} \mathrm{C}\right)$. DSC data were obtained on a TA Instruments (New Castle, DE, USA) mod. Q100 v.9.0 DSC equipped with an intracooler cooling unit at $-25^{\circ} \mathrm{C}$ (with a 1:1 volume mixture of ethylenglycol-water), at a heating 
rate $\beta=20^{\circ} \mathrm{C} / \mathrm{min}$ and at a $\mathrm{N}_{2}: \mathrm{O}_{2}$ ratio of $4: 1(20 \mathrm{~mL} / \mathrm{min})$. Samples were hermetically sealed in aluminium pans, and an empty pan was used as a reference. TG/DTG analyses were conducted with a Perkin-Elmer (Waltham, MA, USA) STA6000 simultaneous thermal analyser by heating the samples in a slow stream of $\mathrm{N}_{2}(20 \mathrm{~mL} / \mathrm{min})$ from room temperature up to $700{ }^{\circ} \mathrm{C}$, with a heating rate of $20^{\circ} \mathrm{C} / \mathrm{min}$. Pyris v.11 software was used for data analysis (PerkinElmer 2014). Temperature calibration was performed with high-grade standards, biphenyl (CRM LGC 2610) and indium (Perkin-Elmer, $\mathrm{x}=99.99 \%$ ), which was also used for enthalpy calibration.

The elemental analysis and vegetal component percentages data used for above calculations, collected from 25 samples of each species with an average height (Carrión-Prieto et al. 2016), is summarized in Table 2. For the determination of ash content, the methodology described in ISO 18122:2015 (ISO 2015) was used, using 5 replicates.

Table 2. Overall chemical composition of C. ladanifer and E. arborea (Carrión-Prieto et al. 2017). Values are given as an average of 25 repetitions, followed by the minimum and maximum values in brackets.

\begin{tabular}{ccc}
\hline & Cistus ladanifer $\mathrm{L}$. & Erica arborea $\mathrm{L}$. \\
\hline Elemental analysis: & & \\
C (\%) & $47.8(47.5-50.1)$ & $51.0(49.3-52.8)$ \\
H (\%) & $6.4(6.0-6.8)$ & $6.2(6.0-6.4)$ \\
N (\%) & $0.8(0.3-1.9)$ & $1.0(0.3-1.1)$ \\
O (by diff., \%) & $\sim 45.0$ & $\sim 41.8$ \\
\hline Cellulose (\%) & $55.0(54.9-55.7)^{\dagger}$ & $40.0(37.3-41.1)$ \\
Lignin (\%) & $25.3(24.5-34.2)$ & $39.5(39.3-40.1)$ \\
Hemi-cellulose (\%) & $10.2(10.1-10.9)^{\ddagger}$ & $11.0(9.7-13.8)^{*}$ \\
Extractive (\%) & $9.5(9.4-9.6)$ & $9.5(5.7-11.0)$ \\
\hline Moisture (wt.\%) & 26.8 & 26.0
\end{tabular}

${ }^{\dagger}$ This cellulose content is higher than that of most woods, which is usually in the $35-50 \%$ range.

* These hemicellulose contents are lower than those of most woods, which usually range from $20 \%$ to $30 \%$.

\section{Results}

\section{HHV values calculated from the elemental analysis data}

Table 3. Carbon $(\mathrm{C})$, hydrogen $(\mathrm{H})$, nitrogen $(\mathrm{N})$ and oxygen $(\mathrm{O})$ percentages for $C$. ladanifer and E. arborea fractions and HHV values calculated thereof.

\begin{tabular}{ccccccccc}
\hline & \multicolumn{4}{c}{ Cistus ladanifer L. } & \multicolumn{3}{c}{ Erica arborea L. } \\
\cline { 2 - 8 } & Leaves & Thin branches & Thick branches & Roots & Leaves & Thin branches & Thick branches & Roots \\
\hline \multirow{2}{*}{$\mathrm{C}(\%)$} & 50.07 & 48.12 & 47.56 & 47.76 & 52.82 & 49.34 & 50.26 & 49.82 \\
& $(0.04)$ & $(0.03)$ & $(0.06)$ & $(0.05)$ & $(0.02)$ & $(0.01)$ & $(0.03)$ & $(0.12)$ \\
H (\%) & 6.4 & 6.4 & 6.4 & 6.4 & 6.2 & 6.2 & 6.2 & 6.2 \\
& $(0.2)$ & $(0.2)$ & $(0.2)$ & $(0.2)$ & $(0.2)$ & $(0.2)$ & $(0.2)$ & $(0.2)$ \\
N (\%) & 1.89 & 0.84 & 0.27 & 0.36 & 1.05 & 0.34 & 0.38 & 0.34 \\
O (by diff.,\%) & $(0.00)$ & $(0.00)$ & $(0.02)$ & $(0.00)$ & $(0.00)$ & $(0.02)$ & $(0.00)$ & $(0.02)$ \\
\hline HHV (kJ·g-1) & 41.64 & 44.60 & 45.77 & 45.48 & 39.93 & 44.12 & 43.16 & 43.64 \\
\hline All & 20.53 & 19.42 & 19.16 & 19.25 & 21.29 & 19.69 & 20.12 & 19.91 \\
\hline
\end{tabular}

All values for the elemental analysis are given in average \pm standard deviations (in brackets) across five replicates. The value for $C$. ladanifer capsules has been omitted due to its low representativeness and to allow comparison of the components of both species. 
HHV values calculated from elemental analysis data according to the IGT formula are reported in Table . HHV values for Cistus ladanifer, from the largest to the smallest, were: foliage (20.53 $\left.\mathrm{kJ} \cdot \mathrm{g}^{-1}\right)$, thin branches $\left(19.42 \mathrm{~kJ} \cdot \mathrm{g}^{-1}\right)$, thick branches $\left(19.16 \mathrm{~kJ} \cdot \mathrm{g}^{-1}\right)$ and roots $\left(19.25 \mathrm{~kJ} \cdot \mathrm{g}^{-1}\right)$. The result for branches was in close agreement with that reported by Dimitrakopoulos and Panov (2001) (viz. $\left.19.05 \mathrm{~kJ} \cdot \mathrm{g}^{-1}\right)$.

HHV values for Erica arborea, from the largest to the smallest, were: foliage $\left(21.29 \mathrm{~kJ} \cdot \mathrm{g}^{-1}\right)$, thick branches $\left(19.69 \mathrm{~kJ} \cdot \mathrm{g}^{-1}\right)$, thin branches $\left(20.12 \mathrm{~kJ} \cdot \mathrm{g}^{-1}\right)$, roots $\left(19.91 \mathrm{~kJ} \cdot \mathrm{g}^{-1}\right)$ and stem wood $(19.8$ $\left.\mathrm{kJ}^{-1} \mathrm{~g}^{-1}\right)$. These results were in reasonably good agreement with those reported by Dimitrakopoulos and Panov (2001) for foliage $\left(23.59 \mathrm{~kJ} \cdot \mathrm{g}^{-1}\right)$ and branches $\left(19.34 \mathrm{~kJ} \cdot \mathrm{g}^{-1}\right)$.

\section{HHV values calculated from the component percentages}

Using Table 3 and the percentage of biomass distribution in each plant (Table 4), overall HHV for both shrubs was readily calculated.

Table 4. Percentage of biomass distribution in each plant (Carrión-Prieto et al. 2017)

\begin{tabular}{ccc}
\hline & \multicolumn{2}{c}{ Component percentage } \\
\cline { 2 - 3 } & Cistus ladanifer L. & Erica arborea L. \\
\hline Leaves (\%) & 19 & 4 \\
Capsules (\%) & 1 & 20 \\
Thin branches (\%) & 29 & 41 \\
Thick branches (\%) & 33 & 35 \\
Roots (\%) & 18 & \\
\hline
\end{tabular}

All values are given in average across 25 samples of each species.

Because C. ladanifer has $19 \%$ of leaves $\left(\times 20.53 \mathrm{~kJ} \cdot \mathrm{g}^{-1}=3.87 \mathrm{~kJ} \cdot \mathrm{g}^{-1}\right), 29 \%$ of thin branches $\left(\times 19.42 \mathrm{~kJ} \cdot \mathrm{g}^{-1}=5.655 \mathrm{~kJ} \cdot \mathrm{g}^{-1}\right), 33 \%$ of thick branches $\left(\times 19.16 \mathrm{~kJ} \cdot \mathrm{g}^{-1}=6.346 \mathrm{~kJ} \cdot \mathrm{g}^{-1}\right)$ and $18 \%$ of roots $\left(\times 19.25 \mathrm{~kJ} \cdot \mathrm{g}^{-1}=3.478 \mathrm{~kJ} \cdot \mathrm{g}^{-1}\right)$, the resultant HHV weighted average was $19.3 \mathrm{~kJ} \cdot \mathrm{g}^{-1}$.

Likewise, E. arborea, with $4 \%$ of leaves $\left(\times 21.29 \mathrm{~kJ} \cdot \mathrm{g}^{-1}=0.855 \mathrm{~kJ} \cdot \mathrm{g}^{-1}\right), 20 \%$ of branches $(\times 19.69$ $\left.\mathrm{kJ} \cdot \mathrm{g}^{-1}=3.952 \mathrm{~kJ} \cdot \mathrm{g}^{-1}\right), 41 \%$ of thick branches $\left(\times 20.12 \mathrm{~kJ} \cdot \mathrm{g}^{-1}=8.28 \mathrm{~kJ} \cdot \mathrm{g}^{-1}\right)$ and $35 \%$ of roots $(\times 19.91$ $\left.\mathrm{kJ} \cdot \mathrm{g}^{-1}=7.0 \mathrm{~kJ} \cdot \mathrm{g}^{-1}\right)$, yielded a HHV weighted average of $20.0 \mathrm{~kJ} \cdot \mathrm{g}^{-1}$.

By applying the weighted average formulas and percentages of bark and wood from Table 1 it was possible to estimate HHV values of $19.5 \mathrm{~kJ} \cdot \mathrm{g}^{-1}$ (bark) and $19.3 \mathrm{~kJ} \cdot \mathrm{g}^{-1}$ (wood) for random C. ladanifer specimens, and of $19.6 \mathrm{~kJ} \cdot \mathrm{g}^{-1}$ (bark) and $19.2 \mathrm{~kJ} \cdot \mathrm{g}^{-1}$ (wood) for old specimens. As regards E. arborea, the bark and wood $\mathrm{HHV}$ values were $20.6 \mathrm{~kJ} \cdot \mathrm{g}^{-1}$ and $19.9 \mathrm{~kJ} \cdot \mathrm{g}^{-1}$, respectively.

If, alternatively, the HHV values were calculated from the maximum holocellulose and lignin+extractives percentages (Table 2.) using the factors proposed by Aseeva et al. (2005) and Kienzle et al. (2001) for such fractions, the results obtained were $20.2 \mathrm{~kJ} \cdot \mathrm{g}^{-1}$ for $C$. ladanifer and $22.1 \mathrm{~kJ} \cdot \mathrm{g}^{-1}$ for E. arborea.

\section{HHV experimental values from calorimetry}

HHV, determined with an isoperibol bomb calorimeter according to the method described in the EN 14918:2009 standard, yielded values of $19.7 \mathrm{~kJ} \cdot \mathrm{g}^{-1}$ for $C$. ladanifer and $21.0 \mathrm{~kJ}^{-1} \mathrm{~g}^{-1}$ for E. arborea. 


\section{Results from DSC and TG/DTG curves}

DSC curves for $C$. ladanifer and E. arborea woods are shown in Fig. S1 and their thermal effects (mainly due to holocellulose and lignin combustion) are summarized in Table 5. Overall enthalpy change values obtained from these curves resulted in $18.04 \mathrm{~kJ} \cdot \mathrm{g}^{-1}$ and $18.63 \mathrm{~kJ} \cdot \mathrm{g}^{-1}$, respectively.

Table 5. Exothermic effects data for holocellulose and lignin in the DSC themograms for C. ladanifer and E. arborea woods

\begin{tabular}{cccccc}
\hline & \multicolumn{2}{c}{ Holocellulose (cellulose+hemicellulose) } & \multicolumn{2}{c}{ Lignin } & Overall enthalpy change \\
\hline & $\mathrm{T}_{\text {peak }}\left({ }^{\circ} \mathrm{C}\right)$ & $\mathrm{T}_{\text {offset }}\left({ }^{\circ} \mathrm{C}\right)$ & $\mathrm{T}_{\text {peak }}\left({ }^{\circ} \mathrm{C}\right)$ & $\mathrm{T}_{\text {offset }}\left({ }^{\circ} \mathrm{C}\right)$ & $\Delta H\left(\mathrm{~kJ} \cdot \mathrm{g}^{-1}\right)$ \\
\hline C. ladanifer & 365 & & 455 & 479 & 18.04 \\
\hline E. arborea & 376 & 392 & 527 & 535 & 18.63 \\
\hline
\end{tabular}

$\mathrm{T}_{\text {peak }}$ stands for the temperature at which the maximum mass loss occurred, according to TG/DTG measurements; $\mathrm{T}_{\text {offset }}$ stands for the temperature at which the maximum value of heat flux occurred, obtained from the DSC thermograms.

The ash content of the various fractions of $C$. ladanifer and E. arborea was estimated from the residue after heating at $700{ }^{\circ} \mathrm{C}$ (Fig. S2 to Fig. S5), according to the usual temperature conditions for pyrolysis in oxygen bomb calorimeters (Wang et al. 2016). Both the inner and outer parts of the stem and those of the epidermis and cortex of the roots of $C$. ladanifer resulted in percentage values ranging from $0.5 \%$ to $0.6 \%$, with no significant differences between fractions. Conversely, for $E$. arborea, the percentages for the stem outer part and the root epidermis ranged from 0.6 to $0.9 \%$, while those for the inner parts of the root and the stem were $0.19 \%$ and $0.36 \%$, respectively. It is worth noting that all these values were below $2 \%$.

\section{Ash content from UNE-EN ISO 18122:2016 method}

Overall experimental ash content values obtained according UNE-EN ISO 18122:2015 norm (ISO 2015) for C. ladanifer and E. arborea were $1.9 \%$ and $1.6 \%$ dry weight, respectively. When the ash percentage was broken down for each of the fractions, for $C$. ladanifer it followed the order: foliage $(9.0 \%)>$ stem bark $(7.0-6.5 \%)>$ roots $(1.4 \%)>$ branches $(1.1 \%)>$ stem wood $(0.7-0.6 \%)$. Likewise, for Erica arborea the AC order followed was: foliage (5.5\%) > stem bark (5.0-4.6\%) > roots $(1.7 \%)>$ branches $(1.1 \%)>$ stem wood $(0.5 \%)$. Simplified data for bark and wood fractions is shown in Table 6.

Table 6. Experimental values for ash content (AC) from bark and wood fractions

\begin{tabular}{cccc}
\hline & Overall AC (\%) & Bark AC (\%) & Wood AC (\%) \\
\hline C. ladanifer L. & 2.45 & 7.0 & 0.7 \\
C. ladanifer L. (old specimens) & 1.9 & 6.5 & 0.6 \\
E. arborea L. & 1.6 & 5.0 & 0.5 \\
\hline
\end{tabular}

Values are given in average across 5 repetitions.

\section{Discussion}

The experimental and calculated HHV values for $C$. ladanifer specimens of indiscriminate age were in the 19.0-19.4 range, as compared to $19.2-20.2 \mathrm{~kJ} \cdot \mathrm{g}^{-1}$ for old specimens. These results were slightly higher than those reported (according to the superseded EN 14775 norm) by García Rosa

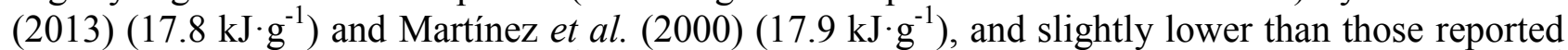
by Marques et al. (2011) $\left(21.4 \mathrm{~kJ} \cdot \mathrm{g}^{-1}\right)$. Analogous results for E. arborea were in the $19.9-22.1 \mathrm{~kJ} \cdot \mathrm{g}^{-1}$ range, in excellent agreement with those reported by Zabaniotou et al. (2000) $\left(20.58 \mathrm{~kJ} \cdot \mathrm{g}^{-1}\right)$ and Tihay et al. (2009) $\left(21.4 \mathrm{~kJ} \cdot \mathrm{g}^{-1}\right)$ and somewhat higher than those reported by Barmpoutis et al. (2015) $\left(19.95 \mathrm{~kJ} \cdot \mathrm{g}^{-1}\right)$. 
Whole enthalpy change values from thermal analysis were around $18.04 \mathrm{~kJ} \cdot \mathrm{g}^{-1}$ and $18.63 \mathrm{~kJ} \cdot \mathrm{g}^{-1}$ for $C$. ladanifer and $E$. arborea, respectively. These values can be assigned to low heating values (LHV), provided that they would be in good agreement with those expected from the holocellulose and lignin net calorific values (ca. $17 \mathrm{~kJ} \cdot \mathrm{g}^{-1}$ and ca. $21 \mathrm{~kJ} \cdot \mathrm{g}^{-1}$, respectively (Energy research Centre of the Netherlands 2012)) and the percentages reported in Table 2.. In fact, the value reported in the literature for the LHV of C. ladanifer is $17.9 \mathrm{~kJ} \cdot \mathrm{g}^{-1}$ (Martínez et al. 2000), very close to the one reported herein.

The ash values obtained for randomly-chosen specimens of $C$. ladanifer according to UNE-EN ISO 18122:2015 (ISO 2015) testing standard were lower than those referred by Ferro et al. (2015) (3.0-3.2\%) and Marques et al. (2011) (whole plant, 3.1\%; wood, 0.8\%), determined according to earlier EN norms, and were in agreement with those informed by Martínez et al. (2000) (2.3\%). For E. arborea, results presented in this study were lower than those reported by Dimitrakopoulos and Panov (2001) (2.5\% for leaves and 1.6\% branches), Doat et al. (1981) (2.4\%) and Boubaker et al. (2004) $(3.5 \%)$.

Regarding the ash content broken down for each of the fractions, the highest value was obtained for stem bark (around 6.0\%), thus identifying this fraction as the one which compromises the use of these shrubs as fuelwood.

In terms of the requirements of ISO 17225-2:2014 (ISO 2014) for ash content of pellets (ENplusB class) and in view of Table 7, C. ladanifer stems with a diameter of $1.9 \mathrm{~cm}$ would be noncompliant, while those with diameters over $3.4 \mathrm{~cm}$ would be acceptable. Consequently, we propose this minimum barked diameter to produce pellets of ENplus-B class. The barked diameter value proposed for E. arborea is entirely coincident with that suggested by Barboutis and Lykidis (2014) following the EN 14961-2 norm.

Table 7. Minimum barked diameter to meet the requirements of ISO 17225-2 norm (ISO 2014) for ash content of pellets and associated HHV values

\begin{tabular}{ccccc}
\hline & Diameter $(\mathrm{cm})$ & Ash $(\%)$ & EN class & HHV $\left(\mathrm{kJ} \cdot \mathrm{g}^{-1}\right)$ \\
\hline C. ladanifer L. & 3.40 & 1.9 & ENplus B & 19.2 \\
E. arborea L. & 2.80 & 1.5 & ENplus B & 19.9 \\
\hline
\end{tabular}

\section{Conclusion}

One of the requirements of current European standards concerning biofuels in the form of pellets for their use in rural district heating is the ash percentage maximum, limited to $2 \%$. Ash content is significantly influenced by the bark and foliage percentages of the plants to be used as fuel. Both shrub species under study, $C$. ladanifer and E. arborea, yielded HHV values that met the requirements established in the regulations for their use as fuel. However, only the ash contents for $E$. arborea were compliant without ambiguity. In the case of $C$. ladanifer, biomass ash percentage was in the upper limit of the normative and this would be a problem for its acceptance as fuelwood. To ensure its adequacy, only old specimens (with stem diameters ranging from 2 to $4.8 \mathrm{~cm}$ ) should be harvested, avoid foliage.

\section{Acknowledgments}

Financial support by the European Union LIFE+ Programme, under project " $\mathrm{CO}_{2}$ Operation: Integrated agroforestry practices and nature conservation against climate change" (ref. LIFE11 $\mathrm{ENV} / \mathrm{ES} / 000535)$, is gratefully acknowledged. 


\section{Electronic Supplementary Material}

DSC, DTA and TG/DTG thermograms for C. ladanifer and E. arborea stems and roots are depicted in Fig. S1 to Fig. S5.
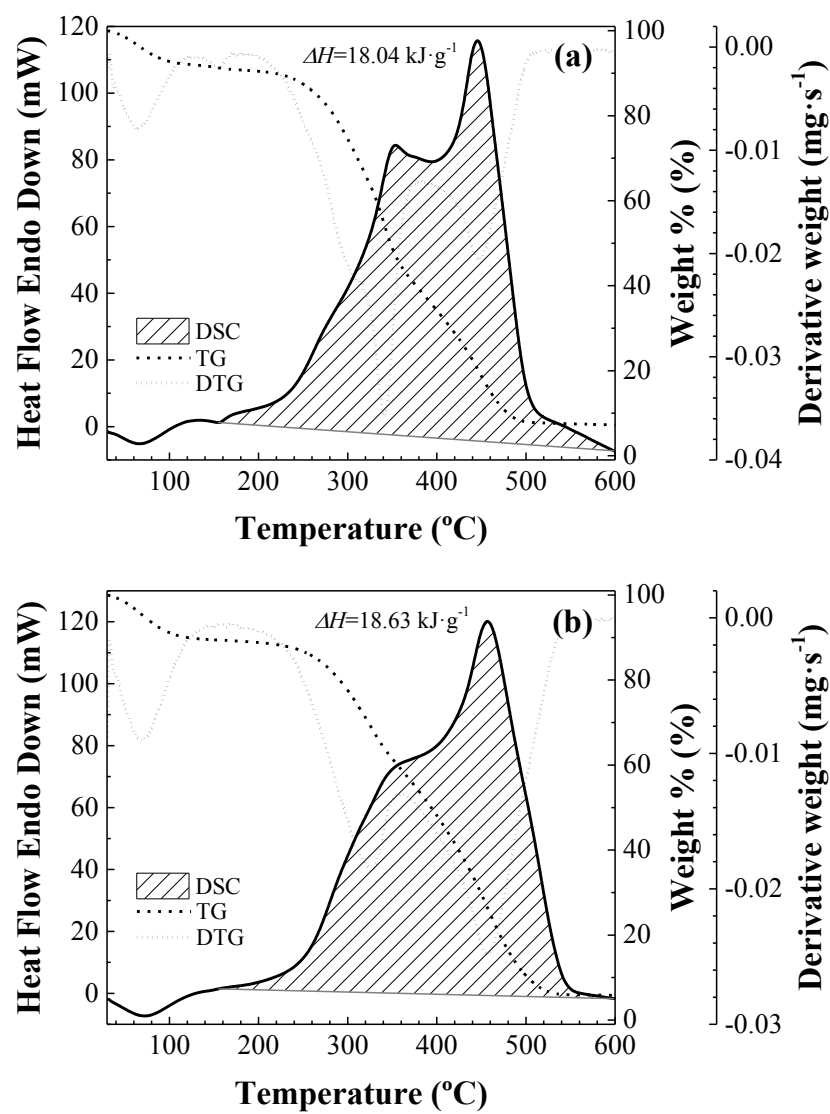

Fig. S1 DSC and TG/DTG curves of (a) C. ladanifer and (b) E. arborea woods. 

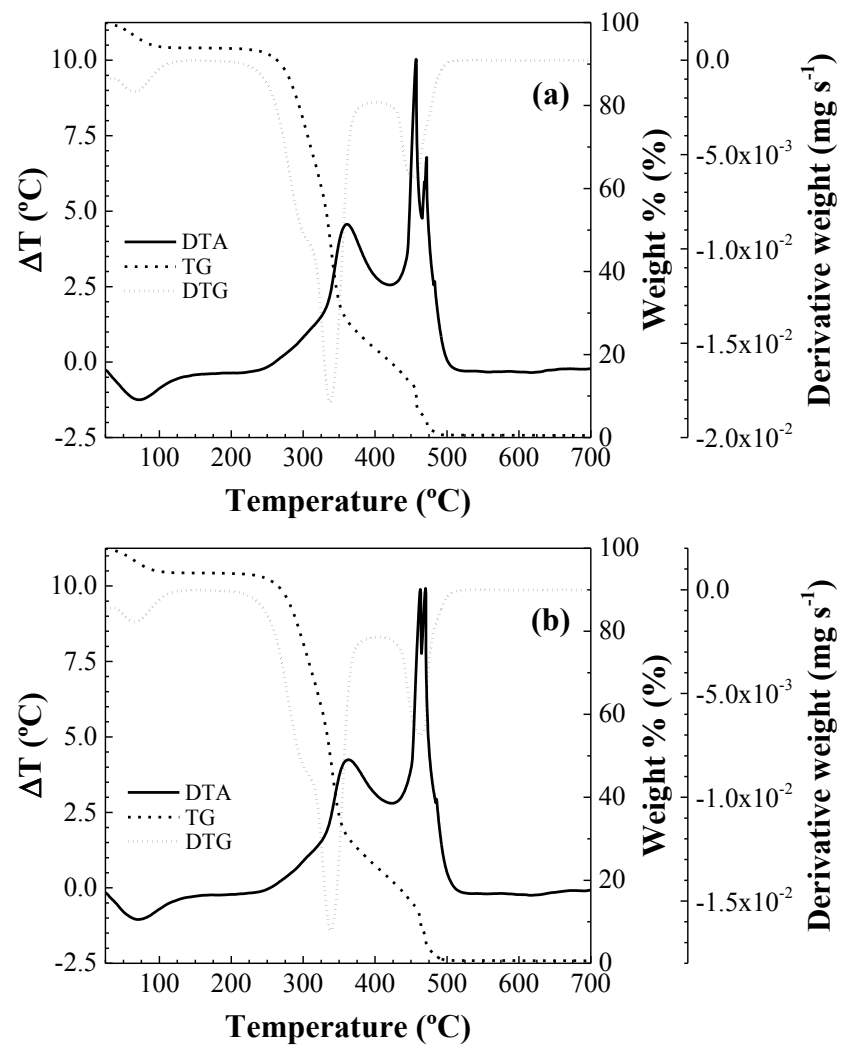

Fig. S2 DTA and TG/DTG curves of $C$. ladanifer stems: (a) external fraction and (b) internal fraction.
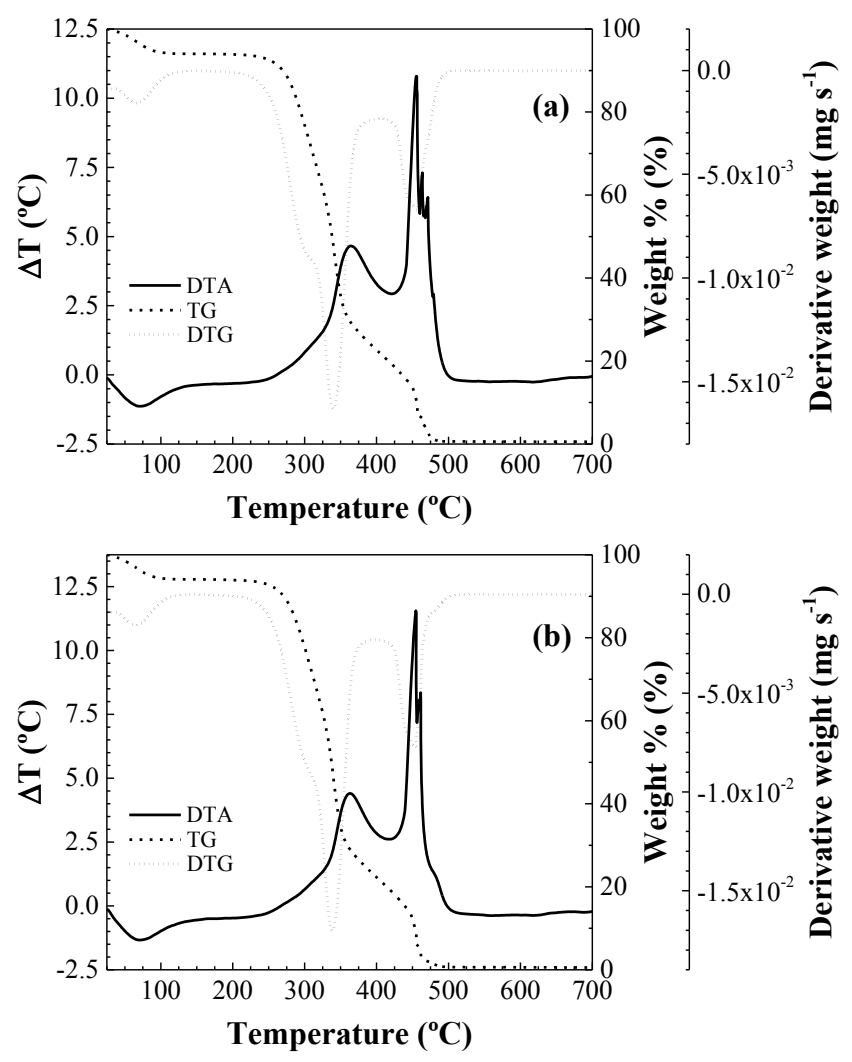

Fig. S3 DTA and TG/DTG curves of $C$. ladanifer roots: (a) epidermis and (b) cortex. 

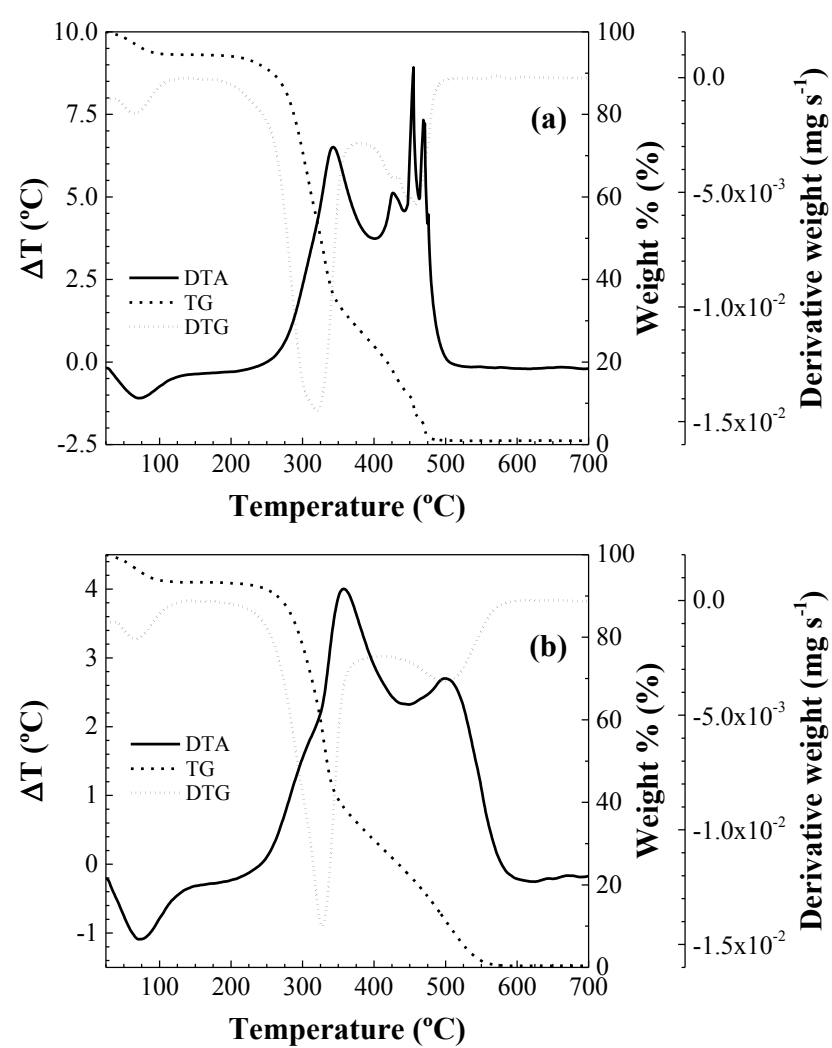

Fig. S4 DTA and TG/DTG curves of E. arborea stems: (a) external fraction and (b) internal fraction.
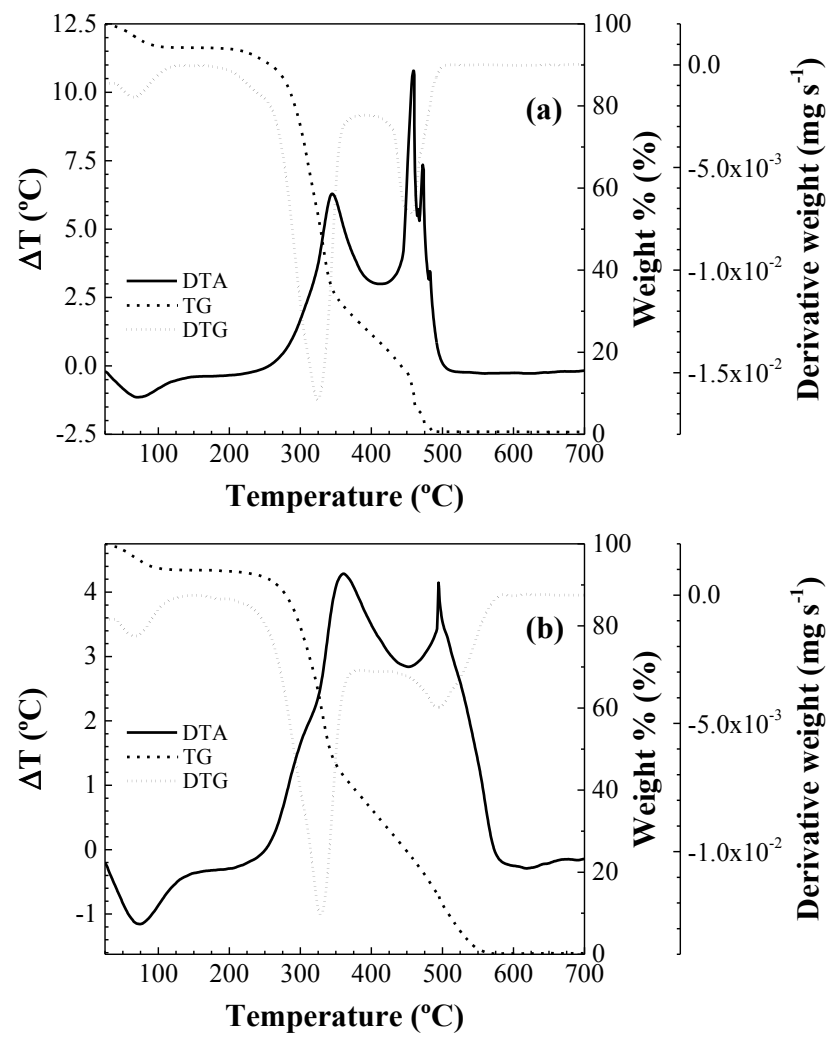

Fig. S5 DTA and TG/DTG curves of E. arborea roots: (a) epidermis and (b) cortex. 


\section{References}

ASEEVA R.M.; B.D. THANH; B.B. SERKOV. 2005. Factors Affecting Heat Release at the Combustion of the Different Species of Wood. In Berlin AA, IA Novakov, NA Khalturinskiy, GE Zaikov eds. Chemical Physics of Pyrolysis, Combustion, and Oxidation. New York, USA. Nova Science Publishers p. 45-53.

BARBOUTIS I.; C. LYKIDIS. Year. The effects of bark on fuel characteristics of some evergreen Mediterranean hardwood species. In Proceedings of the 57th International Convention of Society of Wood Science and Technology, Zvolen, Slovakia. 533-540 p.

BARMPOUTIS P.; C. LYKIDIS; I. BARBOUTIS. 2015. Influence of stem diameter and bark ratio of evergreen hardwoods on the fuel characteristics of the produced pellets. Pro Ligno 11 (4): 673-679

BOMBELLI A.; V. AVITABILE; H. BALZTER; L.B. MARCHESINI; M. BERNOUX; M. BRADY; R. HALL; M. HANSEN; M. HENRY; M. HEROLD; A. JANETOS; B.E. LAW; R. MANLAY; L.G. MARKLUND; H. OLSSON; D. PANDEY; M. SAKET; C. SCHMULLIUS; R. SESSA; Y.E. SHIMABUKURO; R. VALENTINI; M. WULDER. 2009. T12 Assessment of the status of the development of the standards for the Terrestrial Essential Climate Variables: Biomass. Rome, Italy: Global Terrestrial Observing System Secretariat, Land and Water Division (NRL), Food and Agriculture Organization of the United Nations (FAO). p. 30.

BOUBAKER A.; C. KAYOULI; A. BOUKARY; A. BULDGEN. 2004. Chemical and biological characterisation of some woody species browsed by goats in the North-West of Tunisia. In Ben Salem H, P Morand-Fehr, A Nefzaoui eds. Nutrition and feeding strategies of sheep and goats under harsh climates. Zaragoza, Spain. CIHEAM. p. 147-151.

BSI. 2010. BS EN 14918:2009, Solid biofuels. Determination of calorific value. London, UK: British Standards Institution. p. 64.

CARRIÓN-PRIETO P.; S. HERNÁNDEZ-NAVARRO; P. MARTÍN-RAMOS; L.F. SÁNCHEZ-SASTRE; F. GARRIDO-LAURNAGA; J.L. MARCOS-ROBLES; J. MARTÍN-GIL. 2016. Mediterranean shrublands as carbon sinks for Climate Change mitigation: new root to shoot ratios. Carbon Management. DOI: 10.1080/17583004.2017.1285178.

CARRIÓN-PRIETO P.; S. HERNÁNDEZ-NAVARRO; P. MARTÍN-RAMOS; L.F. SÁNCHEZ-SASTRE; F. GARRIDO-LAURNAGA; J.L. MARCOS-ROBLES; J. MARTÍN-GIL. 2017. Mediterranean shrublands as carbon sinks for climate change mitigation: new root-to-shoot ratios. Carbon Management 8 (1): 1-11. DOI: 10.1080/17583004.2017.1285178.

CHPQA. 2008. Guidance Note 29: Alternative fuels - Energy inputs. London, UK: UK Combined Heat \& Power Quality Assurance Programme. p. 6.

DIMITRAKOPOULOS A.P.; P.I. PANOV. 2001. Pyric properties of some dominant Mediterranean vegetation species. International Journal of Wildland Fire 10 (1): 23-17. DOI: 10.1071/wf01003.

DOAT J.; J.C. VALETTE; D. ASKRI; L. CAUMARTIN; M. BETTACHINI; M. MORO. 1981. Le pouvoir calorifique supérieur d'espèces forestières méditerranéennes. Annales des Sciences forestières 38 (4): 469-486

DUCA D.; G. RIVA; E. FOPPA PEDRETTI; G. TOSCANO. 2014. Wood pellet quality with respect to EN 14961-2 standard and certifications. Fuel 135: 9-14. DOI: 10.1016/j.fuel.2014.06.042.

ENERGY RESEARCH CENTRE OF THE NETHERLANDS. 2012. ECN Phyllis2 database for biomass and waste. Consultado 21/01/2017 Disponible en https://www.ecn.nl/phyllis2.

ENPLUS. 2015. ENplus Handbook version 3.0. Brussels, Belgium: European Biomass Association AEBIOM,. p. 103.

EUROPEAN PELLET COUNCIL. 2011. Handbook for the Certification of Wood Pellets for Heating Purposes, based on EN 14961-2. Brussels, Belgium: European Pellet Council. p. 33.

FERRO M.D.; M.C. FERNANDES; A.F.C. PAULINO; S.O. PROZIL; J. GRAVITIS; D.V. EVTUGUIN; A.M.R.B. XAVIER. 2015. Bioethanol production from steam explosion pretreated and alkali extracted Cistus ladanifer (rockrose). Biochemical Engineering Journal 104: 98-105. DOI: 10.1016/j.bej.2015.04.009.

GARCÍA ROSA M. 2013. Estudio de la biomasa de Cistus ladanifer L. y Retama sphaerocarpa L. como sumidero de $\mathrm{CO}_{2}$ : existencias y potencialidad. Tesis PhD. Badajoz, Spain. Departamento de Biología Vegetal, Ecología y Ciencias de la Tierra, Universidad de Extremadura. 230 p. 
ISO. 2014. 17225-2:2014 Solid biofuels, Fuel specifications and classes. Part 2: Graded wood pellets. Geneva, Switzerland: International Organization for Standardization. p. 9.

ISO. 2015. 18122:2015, Solid biofuels, Determination of ash content. Geneva, Switzerland: International Organization for Standardization. p. 6.

KIENZLE E.; I. SCHRAG; R. BUTTERWICK; B. OPITZ. 2001. Calculation of gross energy in pet foods: new data on heat combustion and fibre analysis in a selection of foods for dogs and cats. Journal of Animal Physiology and Animal Nutrition 85 (5-6): 148-157. DOI: 10.1046/j.14390396.2001.00311.x.

MARQUES E.; J.M. PAIVA; C. PINHO. Year. The new Portuguese energy challenge? Pellets from shrubs. In 21st Brazilian Congress of Mechanical Engineering, October 24-28, 2011.'Proceedings'. Natal, RN, Brazil. 12 p.

MARTÍNEZ J.M.; M. VARELA; R. ESCALADA; J.M. MURILLO; E. GONZÁLEZ; J. CARRASCO; P. MANZANARES. Year. Combustion assays of brushwood (Cistus ladanifer) biomass in a BAFB pilot plant. In 1st World Conference on Biomass for Energy and Industry, June 5-9, 2000.'Proceedings'. Sevilla, Spain. 1987-1990 p.

MELLO A.A.D.; L. NUTTO; K.S. WEBER; C.E. SANQUETTA; J.L.M.D. MATOS; G. BECKER. 2012. Individual biomass and carbon equations for Mimosa scabrella Benth. (Bracatinga) in Southern Brazil. Silva Fennica 46 (3): 333-343

MIRANDA I.; I. MIRRA; J. GOMINHO; H. PEREIRA. 2017. Fractioning of bark of Pinus pinea by milling and chemical characterization of the different fractions. Maderas. Ciencia y tecnología (ahead): 0-0. DOI: $10.4067 / \mathrm{s} 0718-221 \times 2017005000016$.

PERKINELMER. 2014. Pyris - Instrument Managing Software, Version 11. Waltham, MA, USA: PerkinElmer, Inc.

RUIZ-PEINADO GERTRUDIX R.; G. MONTERO; M. DEL RIO. 2012. Biomass models to estimate carbon stocks for hardwood tree species. Forest Systems 21 (1): 42-52. DOI: 10.5424/fs/211221102193.

TALWALKAR A.T.; S. UNITED; E. DEPARTMENT OF; T. INSTITUTE OF GAS. 1981. IGT/DOE coalconversion systems technical data book, Chicago, IL, USA. Institute of Gas Technology.

TIHAY V.; P.-A. SANTONI; A. SIMEONI; J.-P. GARO; J.-P. VANTELON. 2009. Skeletal and global mechanisms for the combustion of gases released by crushed forest fuels. Combustion and Flame 156 (8): 1565-1575. DOI: 10.1016/j.combustflame.2009.05.004.

VALARES MASA C.; T. SOSA DÍAZ; J. ALÍAS GALLEGO; N. CHAVES LOBÓN. 2016. Quantitative variation of flavonoids and diterpenes in leaves and stems of Cistus ladanifer L. at different ages. Molecules 21 (3): 275-288. DOI: 10.3390/molecules21030275.

WANG F.; L.J. HU; Y.W. ZHENG; Y.B. HUANG; X.Q. YANG; C. LIU; J. KANG; Z.F. ZHENG. 2016. Regulation for Optimal Liquid Products during Biomass Pyrolysis: A Review. IOP Conference Series: Earth and Environmental Science 40: 012047. DOI: 10.1088/1755-1315/40/1/012047.

ZABANIOTOU A.A.; A.I. ROUSSOS; C.J. KORONEOS. 2000. A laboratory study of cotton gin waste pyrolysis. Journal of Analytical and Applied Pyrolysis 56 (1): 47-59. DOI: 10.1016/s01652370(00)00088-7. 
5.3. Publicación \#3:

VIBRATIONAL AND THERMAL STUDIES OF ESSENTIAL OILS DERIVED FROM CISTUS LADANIFER AND ERICA ARBOREA SHRUBS (NATURAL PRODUCT COMMUNICATIONS, 2017, 12(1):119-122)

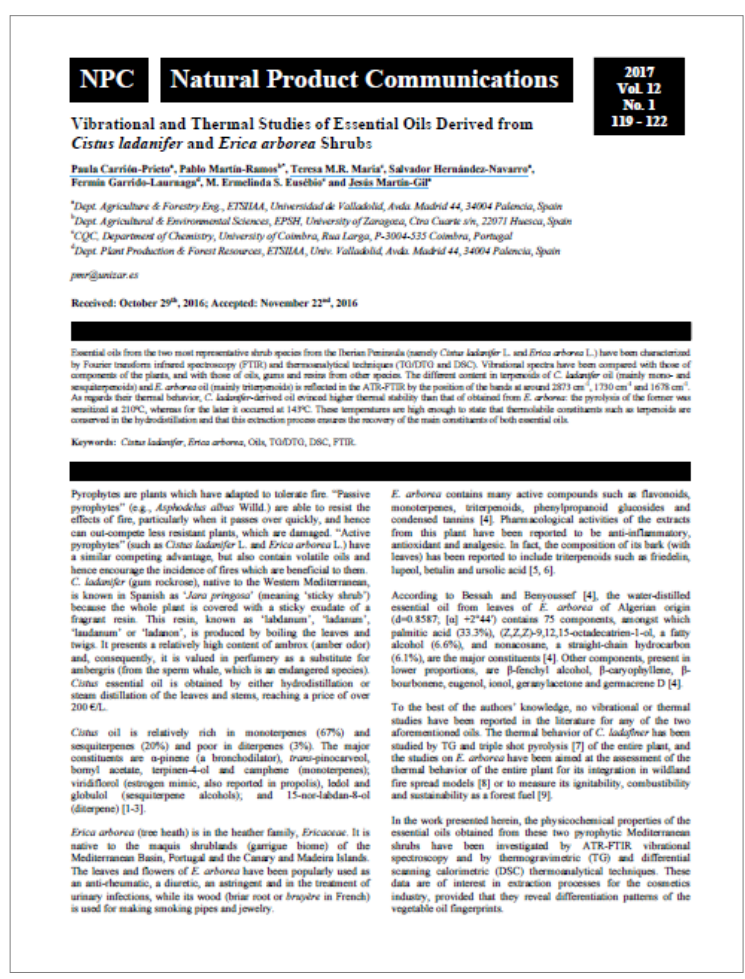



Natural Product Communications

\title{
Vibrational and Thermal Studies of Essential Oils Derived from Cistus ladanifer and Erica arborea Shrubs
}

\author{
Paula Carrión-Prieto ${ }^{a}$, Pablo Martín-Ramos ${ }^{b^{*}}$, Teresa M.R. Maria ${ }^{c}$, Salvador Hernández-Navarro ${ }^{a}$, \\ Fermín Garrido-Laurnaga $^{d}$, M. Ermelinda S. Eusébio ${ }^{c}$ and Jesús Martín-Gil ${ }^{\mathrm{a}}$ \\ ${ }^{a}$ Dept. Agriculture \& Forestry Eng., ETSIIAA, Universidad de Valladolid, Avda. Madrid 44, 34004 Palencia, \\ Spain \\ ${ }^{\mathrm{b}}$ Dept. Agricultural \& Environmental Sciences, EPSH, University of Zaragoza, Ctra Cuarte $s / n, 22071$ \\ Huesca, Spain \\ ${ }^{\mathrm{c}}$ CQC, Department of Chemistry, University of Coimbra, Rua Larga, P-3004-535 Coimbra, Portugal. \\ ${ }^{\mathrm{d} D e p t . ~ P l a n t ~ P r o d u c t i o n} \&$ Forest Resources, ETSIIAA, Univ. Valladolid, Avda. Madrid 44, 34004 Palencia, \\ Spain
}

Received: October $29^{\text {th }}, 2016$; Accepted: November $22^{\text {nd }}, 2016$

Essential oils from the two most representative shrub species from the Iberian Peninsula (namely Cistus ladanifer L. and Erica arborea L.) have been characterized by Fourier transform infrared spectroscopy (FTIR) and thermoanalytical techniques (TG/DTG and DSC). Vibrational spectra have been compared with those of components of the plants, and with those of oils, gums and resins from other species. The different content in terpenoids of $C$. ladanifer oil (mainly mono- and sesquiterpenoids) and E. arborea oil (mainly triterpenoids) is reflected in the ATR-FTIR by the position of the bands at around $2873 \mathrm{~cm}^{-1}, 1730 \mathrm{~cm}^{-1}$ and $1678 \mathrm{~cm}^{-1}$. As regards their thermal behavior, C. ladanifer-derived oil evinced higher thermal stability than that of obtained from E. arborea: the pyrolysis of the former was sensitized at $210^{\circ} \mathrm{C}$, whereas for the later it occurred at $143^{\circ} \mathrm{C}$. These temperatures are high enough to state that thermolabile constituents such as terpenoids are conserved in the hydrodistillation and that this extraction process ensures the recovery of the main constituents of both essential oils.

Keywords: Cistus ladanifer L., Erica arborea L., Oils, TG/DTG, DSC, FTIR.

Pyrophytes are plants which have adapted to tolerate fire. "Passive pyrophytes" (e.g., Asphodelus albus Willd.) are able to resist the effects of fire, particularly when it passes over quickly, and hence can out-compete less resistant plants, which are damaged. "Active pyrophytes" (such as Cistus ladanifer L. and Erica arborea L.) have a similar competing advantage, but also contain volatile oils and hence encourage the incidence of fires which are beneficial to them.

C. ladanifer (gum rockrose), native to the Western Mediterranean, is known in Spanish as 'Jara pringosa' (meaning 'sticky shrub') because the whole plant is covered with a sticky exudate of a fragrant resin. This resin, known as 'labdanum', 'ladanum', 'laudanum' or 'ladanon', is produced by boiling the leaves and twigs. It presents a relatively high content of ambrox (amber odor) and, consequently, it is valued in perfumery as a substitute for ambergris (from the sperm whale, which is an endangered species). Cistus essential oil is obtained by either hydrodistillation or steam distillation of the leaves and stems, reaching a price of over $200 € / \mathrm{L}$.

Cistus oil is relatively rich in monoterpenes $(67 \%)$ and sesquiterpenes $(20 \%)$ and poor in diterpenes $(3 \%)$. The major constituents are $\alpha$-pinene (a bronchodilator), trans-pinocarveol, bornyl acetate, terpinen-4-ol and camphene (monoterpenes); viridiflorol (estrogen mimic, also reported in propolis), ledol and globulol (sesquiterpene alcohols); and 15-nor-labdan-8-ol (diterpene) [1-3].

Erica arborea (tree heath) is in the heather family, Ericaceae. It is native to the maquis shrublands (garrigue biome) of the Mediterranean Basin, Portugal and the Canary and Madeira Islands. The leaves and flowers of E. arborea have been popularly used as an anti-rheumatic, a diuretic, an astringent and in the treatment of urinary infections, while its wood (briar root or bruyère in French) is used for making smoking pipes and jewelry.

E. arborea contains many active compounds such as flavonoids, monoterpenes, triterpenoids, phenylpropanoid glucosides and condensed tannins [4]. Pharmacological activities of the extracts from this plant have been reported to be anti-inflammatory, antioxidant and analgesic. In fact, the composition of its bark (with leaves) has been reported to include triterpenoids such as friedelin, lupeol, betulin and ursolic acid $[5,6]$.

According to Bessah and Benyoussef [4], the water-distilled essential oil from leaves of E. arborea of Algerian origin $\left(d=0.8587 ;[\alpha]+2^{\circ} 44^{\prime}\right)$ contains 75 components, amongst which palmitic acid (33.3\%), (Z,Z,Z)-9,12,15-octadecatrien-1-ol, a fatty alcohol $(6.6 \%)$, and nonacosane, a straight-chain hydrocarbon $(6.1 \%)$, are the major constituents [4]. Other components, present in lower proportions, are $\beta$-fenchyl alcohol, $\beta$-caryophyllene, $\beta$-bourbonene, eugenol, ionol, geranylacetone and germacrene D [4].

To the best of the authors' knowledge, no vibrational or thermal studies have been reported in the literature for any of the two aforementioned oils. The thermal behavior of $C$. ladafiner has been studied by TG and triple shot pyrolysis [7] of the entire plant, and the studies on E. arborea have been aimed at the assessment of the thermal behavior of the entire plant for its integration in wildland fire spread models [8] or to measure its ignitability, combustibility and sustainability as a forest fuel [9].

In the work presented herein, the physicochemical properties of the essential oils obtained from these two pyrophytic Mediterranean shrubs have been investigated by ATR-FTIR vibrational spectroscopy and by thermogravimetric (TG) and differential scanning calorimetric (DSC) thermoanalytical 
techniques. These data are of interest in extraction processes for the cosmetics industry, provided that they reveal differentiation patterns of the vegetable oil fingerprints.

ATR-FTIR spectra: The wavenumbers for the main bands in the ATR-FTIR spectra of the oils of the two plants under study, together with those from leaves, roots, capsules, fine and coarse-texture components for each of the species, are summarized in Table 1. A close similarity can be observed, on the one hand, between the leaves and roots spectra and, on the other hand, between the fine and coarse components spectra. The capsules vibrational pattern for E. arborea exactly matched that of the fine components and is listed in the same column.

Table 1: Main bands in the ATR-FTIR vibrational spectra of $C$. ladanifer and $E$. arborea oils and various plant components. All wavenumber values are in $\mathrm{cm}^{-1}$.

\begin{tabular}{|c|c|c|c|c|c|c|c|c|c|}
\hline \multicolumn{5}{|c|}{ Cistus ladanifer } & \multicolumn{5}{|c|}{ Erica arborea } \\
\hline Oil & Leaves & Roots & Fine & Coarse & Oil & Leaves & Roots & Capsules / Fine & Coarse \\
\hline 3465 & 3412 & 3416 & 3419 & 3421 & 3360 & 3396 & 3405 & 3421 & 3412 \\
\hline 2953 & $x$ & x & $\times$ & $\times$ & 2970 & $\times$ & $x$ & $\times$ & $\times$ \\
\hline 2918 & 2919 & 2920 & 2922 & 2920 & 2931 & 2923 & 2929 & 2918 & 2919 \\
\hline 2871 & 2850 & x & & $x$ & 2874 & 2852 & $x$ & 2850 & \\
\hline$\times$ & 2360 & 2361 & 2355 & 2361 & $\times$ & 2360 & 2361 & 2360 & 2360 \\
\hline$x$ & $\neq$ & 2344 & 2339 & 2343 & $x$ & $\neq$ & 2344 & 2342 & 2343 \\
\hline 1737 & 1733 & 1735 & 1732 & 1735 & 1721 & 1733 & 1736 & 1739 & 1740 \\
\hline 1681 & $\times$ & $\times$ & $\times$ & x & 1674 & $\times$ & $\times$ & $\times$ & $\times$ \\
\hline$\times$ & 1615 & 1617 & 1621 & 1617 & 1614 & 1616 & 1617 & 1616 & 1616 \\
\hline$x$ & 1515 & 1508 & 1505 & 1508 & $\times$ & 1516 & 1513 & 1508 & 1510 \\
\hline 1448 & 1455 & 1452 & 1455 & 1457 & 1454 & 1455 & 1451 & 1464 & 1459 \\
\hline 1375 & 1367 & 1375 & 1373 & 1375 & 1374 & 1373 & 1374 & 1375 & 1375 \\
\hline 1328 & $\neq$ & 1320 & & 1320 & 1329 & $\neq$ & & 1331 & \\
\hline 1245 & 1232 & 1243 & 1242 & 1243 & 1249 & 1243 & 1247 & 1246 & 1247 \\
\hline 1164 & 1164 & & & & 1152 & & 1151 & & \\
\hline 1125 & & & & & & & & & \\
\hline 1111 & & & & & 1085 & & 1081 & & \\
\hline$\times$ & 1035 & 1035 & 1031 & 1035 & 1046 & 1035 & 1031 & 1050 & 1039 \\
\hline 1015 & & & & & 1007 & & & & \\
\hline 952 & & & & & 939 & & & & \\
\hline 886 & & & & & 860 & & & & \\
\hline 815 & & & & & 821 & & & & \\
\hline 787 & & & & & 758 & & & & \\
\hline 635 & & & & & 665 & & & & \\
\hline 618 & 617 & 559 & 602 & 610 & 559 & & 579 & 608 & 607 \\
\hline
\end{tabular}

The oils spectra differed from those of the leaves, roots and other plant components from which they were obtained by the absence of the absorption bands at $2360 \mathrm{~cm}^{-1}$ (absorbed $\mathrm{CO}_{2}$ ), $2344 \mathrm{~cm}^{-1}$ (glucose ring stretching), $1515 \mathrm{~cm}^{-1}$ (benzene ring stretching in lignin) and $1035 \mathrm{~cm}^{-1}$ (lignin), present in the latter components. Nonetheless, they all shared the presence of bands at $1455 \mathrm{~cm}^{-1}$
$\left(\mathrm{CH}_{2}\right.$ cellulose, lignin) and $1375 \mathrm{~cm}^{-1}$ (C-H cellulose, hemicellulose) [10]. Bands specific to the oils are those which appeared at 2960, 2873, 1678, 945 and $818 \mathrm{~cm}^{-1}$. The bands at 2960 and $2873 \mathrm{~cm}^{-1}$ are assigned to asymmetrically and symmetrically stretching vibration of $\mathrm{C}-\mathrm{H}$ of aliphatic $\mathrm{CH}_{3}$ groups, due to the alkyl rest of the triglycerides present in large quantities in vegetable oils, while the band at around $1678 \mathrm{~cm}^{-1}$ can either be attributed to $\mathrm{C}=\mathrm{C}$ stretching vibration of cis disubstituted olefins $(\mathrm{RHC}=\mathrm{CHR})$ or to an oxo group $(\alpha: \beta$ unsaturated) from terpenoids. The $\mathrm{C}-\mathrm{H}$ out-of-plane deformation band observed between 952 and $939 \mathrm{~cm}^{-1}$ is highly characteristic of trans double bonds, and the band at around $818 \mathrm{~cm}^{-1}$ is related to an isopropylidine group $\left(\mathrm{R}_{2} \mathrm{C}=\mathrm{CHR}\right)$, usual in terpenoids [1114].

The fact that the labdanum and tree heath oils spectra (Figure 1 ), as is the case for other oils, showed bands in common with resins and gums has led us to compare these spectra with those of rosehip and palm oils, myrrh and mastic resins and tragacanth gum (see Table 2). It is worth noting that, in the $2920-1160 \mathrm{~cm}^{-1}$ region, there is a high correspondence with the bands of $R$. rubiginosa oil, a moderate correspondence with those of other oils and resins, and low agreement with those of tragacanth gum.

In the case of $C$. ladanifer oil, whose terpene composition is favorable to low-number isoprenic units (monoterpenes and sesquiterpenes), the band at $1737 \mathrm{~cm}^{-1}$ (attributable to $v(\mathrm{CO})$ ester carbonyl or to terpenic oxo groups) is shared with rosehip and palm oils, whereas the composition rich in terpenes with a high number of isoprenic units (triterpenoids) exhibited by $E$. arborea oil leads to a shift of this band towards lower wavenumbers $\left(1721 \mathrm{~cm}^{-1}\right)$. As regards the band at around 2875 $\mathrm{cm}^{-1}$ (attributed to symmetrical $v(\mathrm{C}-\mathrm{H})$ from $\mathrm{CH}_{3}$ ) in the $E$. arborea spectrum, it should be noted that it agrees with that of mastic resin. Another useful band in terms of differential identification of the oils is that which appears at $1681 \mathrm{~cm}^{-1}$ in $C$. ladanifer oil and shifted to lower wavenumbers in tree heath oil $\left(1674 \mathrm{~cm}^{-1}\right)$ and rosehip oil $\left(1653 \mathrm{~cm}^{-1}\right)$. This band does not appear in palm pulp oil or in tragacanth gum.

Table 2: Comparison of the vibrational spectra of $C$. ladanider and E. arborea oils with rosehip and palm oils, myrrh and mastic resins and tragacanth gum.

\begin{tabular}{|c|c|c|c|c|c|c|c|c|c|}
\hline \multicolumn{2}{|c|}{ C. ladanifer } & \multicolumn{2}{|c|}{ E. arborea } & \multirow{2}{*}{ Myrrh resin } & \multirow{2}{*}{ Mastic resin } & \multirow{2}{*}{ Rosehip oil [15] } & \multirow{2}{*}{ Palm pulp oil } & \multirow{2}{*}{ Tragacanth gum } & \multirow{2}{*}{ Assignments } \\
\hline extract & oil & extract & oil & & & & & & \\
\hline & 3465 & & & & & & & & $\mathrm{OH}$ group \\
\hline 3362 & & 3366 & 3360 & 3348 & & & & 3335 & \\
\hline 2969 & 2953 & 2969 & 2970 & & 2949 & 3009 & 3004 & & asymmetric $v(\mathrm{CH})$ from $\mathrm{CH}_{3}$ \\
\hline \multirow[t]{2}{*}{2915} & 2918 & 2930 & 2931 & 2925 & & 2923 & 2914 & 2932 & asymmetric $v(\mathrm{CH})$ from $\mathrm{CH}_{2}$ \\
\hline & 2871 & 2875 & 2874 & & 2874 & & & & symmetric $v(\mathrm{CH})$ from $\mathrm{CH}_{3}$ \\
\hline 2848 & 2834 & & & 2854 & & 2853 & 2850 & & \\
\hline 1732 & 1737 & 1735 & 1721 & 1732 & & 1742 & 1736 & 1716 & $v(\mathrm{CO})$ ester carbonyl of triglycerides \\
\hline 1712 & 1710 & & & & 1707 & & & & \\
\hline 1655 & 1681 & 1674 & 1674 & 1635 & 1650 & 1653 & $x$ & $\times$ & $v(\mathrm{C}=\mathrm{C})$ disubstituted olefins; olefinic terpenoids \\
\hline 1607 & & 1614 & 1614 & 1601 & & & & 1600 & $v(\mathrm{C}=\mathrm{C})$ aromatic ring \\
\hline \multirow[t]{2}{*}{1462} & 1448 & 1454 & 1454 & & 1459 & 1456 & 1467 & & $\delta\left(\mathrm{CH}_{2}\right)$ bending deformation \\
\hline & & & & 1435 & & & & 1410 & $\delta(\mathrm{C}-\mathrm{H})$ from $\mathrm{CH}_{2}$ or $\mathrm{CH}_{3}$ groups; $\mathrm{CH}_{2}$ cellulose/lignin \\
\hline 1378 & 1375 & 1374 & 1374 & 1378 & & 1377 & 1389 & 1371 & $\begin{array}{c}\delta\left(\mathrm{CH}_{2}\right)_{2} \text { bending deformation } \\
\mathrm{CH} \text { hemicellulose, cellulose }\end{array}$ \\
\hline 1341 & 1328 & 1329 & $\begin{array}{l}1328 \\
1298\end{array}$ & 1335 & & & & $\times$ & $\delta\left(\mathrm{CH}_{3}\right)$ bending deformation \\
\hline 1227 & 1245 & 1249 & 1249 & 1238 & 1245 & 1238 & 1243 & 1242 & $\delta(\mathrm{C}-\mathrm{H}), v(\mathrm{C}-\mathrm{O}-\mathrm{H})$ \\
\hline 1160 & 1164 & 1152 & 1142 & & 1161 & 1160 & 1170 & 1145 & methyl ester, $v(\mathrm{CO})$ \\
\hline 1128 & 1125 & & & & & & & 1120 & $v(\mathrm{C}-\mathrm{C})$ \\
\hline \multirow[t]{5}{*}{1106} & 1111 & & & & 1115 & & & & \\
\hline & 1083 & 1085 & 1084 & & & 1098 & 1100 & & stretching vibration of $\mathrm{C}-\mathrm{O}$ ester groups \\
\hline & 1048 & 1046 & 1045 & & 1046 & & & & starch $\mathrm{OH}$, cellulose \\
\hline & 1032 & & & 1023 & & & & 1034 & \\
\hline & 993 & 1007 & 1007 & & 1008 & & & & trans double bond \\
\hline 950 & 952 & 939 & 988 & & & & & & $\delta(\mathrm{C}-\mathrm{H})$ bending \\
\hline 876 & 886 & 860 & 860 & 841 & 837 & & & 892 & $\delta(\mathrm{C}-\mathrm{O}-\mathrm{C})$ \\
\hline \multirow[t]{2}{*}{816} & 815 & 821 & 838 & & & & & 846 & $\mathrm{R}_{2} \mathrm{C}=\mathrm{CHR}$ isopropylidine \\
\hline & 787 & & & & & & & & \\
\hline 729 & & 758 & 758 & 725 & & 723 & 717 & $x$ & $\rho\left(\mathrm{CH}_{2}\right)_{\mathrm{n}}$ \\
\hline 719 & 698 & & 701 & & & & & & 1,2-cis-disubstituted olefin \\
\hline \multirow[t]{3}{*}{638} & 635 & 665 & 662 & 597 & 580 & & & & $\delta(\mathrm{C}-\mathrm{H})$ in the furan ring \\
\hline & 563 & 559 & 559 & & & & & 595 & \\
\hline & & & 527 & & & & & 521 & \\
\hline
\end{tabular}



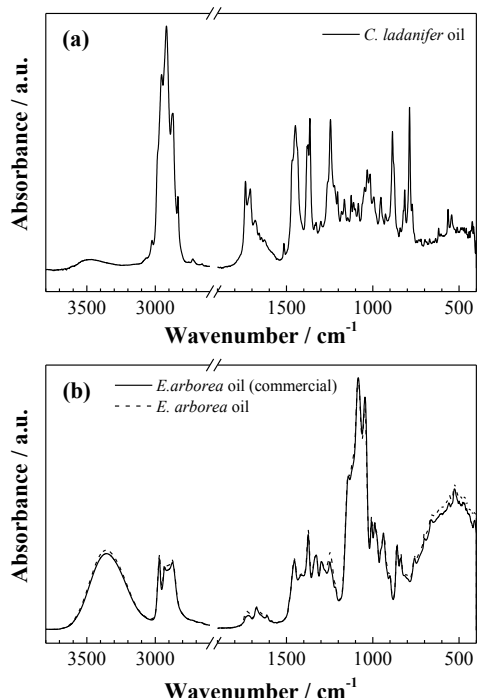

Figure 1: FTIR spectra of (a) Cistus ladanifer oil and (b) Erica arborea oil

Thermal analysis: TG plots of gum rockrose and tree heath oils showed mass loss in the $50-240^{\circ} \mathrm{C}$ and $100-220^{\circ} \mathrm{C}$ temperature range, respectively, with a more abrupt pattern for E. arborea (Figure 2). In both cases this mass loss corresponded to pyrolysis, which was sensitized in the DTG curves by endotherms at $151^{\circ} \mathrm{C}(C$. ladanifer $)$ and at $208^{\circ} \mathrm{C}(C$. arborea $)$. From these temperatures, it can be seen that $E$. arborea oil presents higher thermal stability than that of $C$. ladanifer. In any case, the decomposition point of both oils is high enough to ensure the preservation of terpenoids as main constituents. The chief products of pyrolysis were straight-chain alkanes and alkenes [16]. It should be noted that heating of isopropanol extracts (not shown) resulted, after solvent evaporation, in decomposition of the oils extending up to $400{ }^{\circ} \mathrm{C}$.

The low-temperature DSC thermograms of the two oils under study are depicted in Figure 3. Both C. ladanifer and E. arborea oils vitrify upon cooling. Upon heating, only devitrification is observed. For $C$. ladanifer oil the glass transition is observed at $T_{\mathrm{g}}=(-113 \pm 1)^{\circ} \mathrm{C}$ and for $E$. arborea oil at $T_{\mathrm{g}}=(-79 \pm 1)^{\circ} \mathrm{C}$.
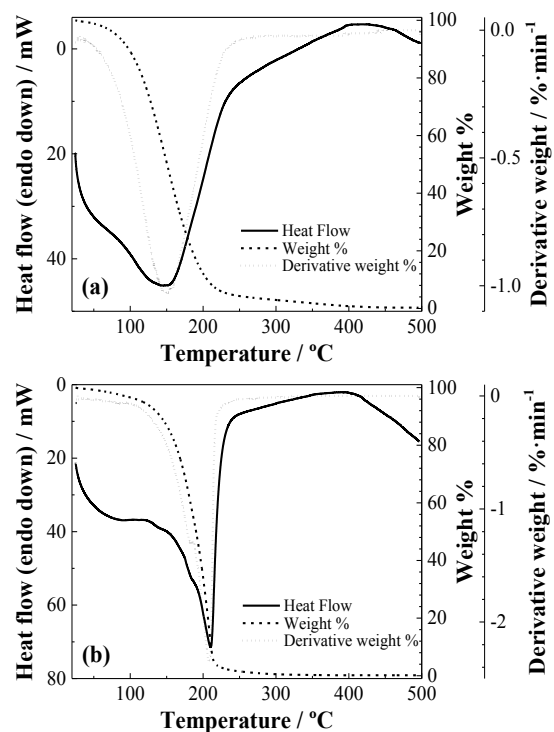

Figure 2: Thermograms for $(a) C$. ladanifer oil, $m=21.14 \mathrm{mg}$; and $(b) E$. arborea oil, $\mathrm{m}=17.77 \mathrm{mg}$. The left $y$ axis corresponds to the DSC curve, while the two $y$ axes on the right correspond to TG/DTG curves.
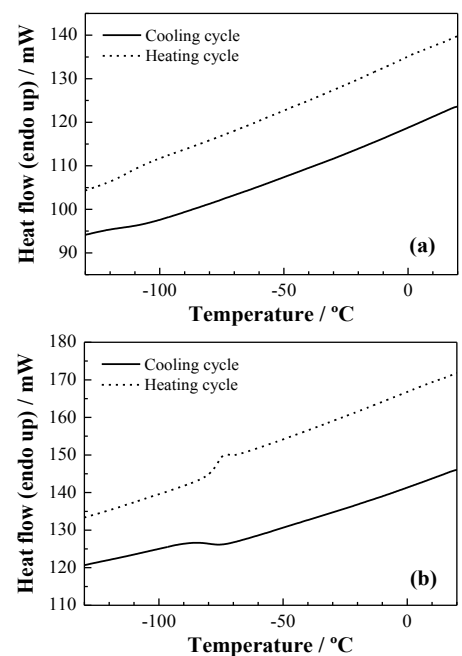

Figure 3: Low-temperature DSC thermograms for (a) C. ladanifer oil, $\mathrm{m}=$ $4.1 \mathrm{mg}$; and (b) E. arborea oil, $\mathrm{m}=5.7 \mathrm{mg}$. The cooling/heating rate was $|\beta|=50^{\circ} \mathrm{C} \cdot \mathrm{min}^{-1}$.

As regards the FTIR results, this technique has proven useful to identify the different components of various parts of the plants and their derived products, serving as a fingerprint technique which provides insight into the biochemical composition of the samples. As noted by Huck [17], this method is particularly suitable for the fast and simultaneous qualitative and quantitative characterization of natural products and their constituents. Thus, the data presented herein can be used for quality control in the cosmetics industry.

With regard to the thermal stability of the oils under study, it is high enough to ensure that their thermolabile constituents would be preserved during hydro-distillation, which is the method recommended by the French Pharmacopoeia for the extraction of essential oils from dried spices and the quality control of essential oils in the laboratory [18].

On another topic, provided that the two shrub species under study are active pyrophytes, the results should also be put in relation to their impact on forest fires: the flammability of the shrubs under study (and, by extension, those of their resins and oils) must be referred (apart from ignition time and moisture) to their contents of terpenoids. The high flammability of $E$. arborea can be ascribed to its high emission of terpenes throughout all the year (which can reach up to $40 \mu \mathrm{g} \cdot \mathrm{g}_{\mathrm{DM}}{ }^{-1} \cdot \mathrm{h}^{-1}$ ), in spite of the fact that its composition is relatively rich in lowvolatile terpenoids, such as triterpenoids. Conversely, although C. ladanifer emits into the air modest amounts of terpenoids $(<7$ $\left.\mu \mathrm{g} \cdot \mathrm{g}_{\mathrm{DM}}{ }^{-1} \cdot \mathrm{h}^{-1}\right)$, it has a very high flammability in the summer due to the potentiated emission of $\alpha$-pinene, a particularly volatile monoterpene, which, as noted above, is an important component of its essential oil [19].

The essential oils from two Western Mediterranean pyrophytes, namely $C$. ladanifer and E. arborea, have been characterized by ATR-FTIR spectroscopy and thermal analytical (TG/DTG and DSC) techniques. Their vibrational spectra have been compared with those of other parts of the plants (leaves, roots, capsules, etc.) and with those of oils, gums and resins from other species. The specific location of the bands from unsaturated and $\alpha: \beta$ unsaturated oxogroups has been related to the different content of terpenoids of $C$. ladanifer oil (mono- and sesquiterpenoids) and E. arborea (triterpenoids). As regards the thermal behavior, $E$. arborea oil showed higher thermal stability than that of $C$. ladanifer, as evinced by the effects in the TG and DSC thermograms (at $210^{\circ} \mathrm{C}$ and $143^{\circ} \mathrm{C}$, respectively). Thus, recognition of the TG and DSC characteristic patterns of both essential oils can also be helpful in identifying the type of oil. On the other hand, the delayed thermal decomposition of the oils under study, together with the characterization of 
terpenoids by ATR-FTIR, provide evidence that oil constituents that are thermolabile, such as terpenoids, are conserved in the extraction process, thus ensuring that hydrodistillation is a valuable method to recover the main constituents of these essential oils.

\section{Experimental}

Samples: C. ladanifer and E. arborea samples were collected from wild plants growing in the municipality of Ayoó de Vidriales (in the province of Zamora, Castilla y Leon, Spain) during the flowering period (Spring 2015). Oils from Rosa rubiginosa L. and Elaeis guineensis Jacq., myrrh from Commiphora, mastic from Pistacia lentiscus L. and tragacanth gum from Astragalus samples used for comparison purposes were of commercial origin.

Methods: Hydro-distillation of $100 \mathrm{~g}$ of fresh and whole leaves of C. ladanifer and E. arborea was carried out in a Clevenger-type apparatus for $3 \mathrm{~h}$. The essential oils were collected, dried under anhydrous sodium sulfate and stored at $4^{\circ} \mathrm{C}$ until used. To confirm their purity, essential oil composition was determined by gas chromatography coupled to mass spectrometric (GC-MS) analysis, according to the experimental conditions specified by Bessah and Benyoussef [4]. A commercial sample E. arborea essential oil, supplied by Radhe Shyam (Barcelona, Spain), was also tested. An alternative extraction method consisting of suspending the vegetal dry matter in amyl alcohol in a 1:2 (w/v) ratio for $30 \mathrm{~min}$, under constant shaking, at room temperature, was also used to isolate high-purity oil from both plants.

Apparatus: GC/MS analysis was conducted with an Agilent Technologies 7890A apparatus (Santa Clara, CA, USA). The vibrational spectra of the materials in the $400-4000 \mathrm{~cm}^{-1}$ spectral range were measured using a Thermo Scientific (Waltham, MA, USA) Nicolet iS50 FT-IR spectrometer, equipped with an in-built diamond attenuated total reflection (ATR) system. Spectra of the oils were recorded at room temperature with a $1 \mathrm{~cm}^{-1}$ spectral resolution, and 64 scans. TG and high-temperature DSC analyses were conducted with a Perkin-Elmer (Waltham, MA, USA) STA6000 simultaneous thermal analyser by heating the samples in a slow stream of $\mathrm{N}_{2}$ $\left(20 \mathrm{~mL} \cdot \mathrm{min}^{-1}\right)$ from room temperature up to $500^{\circ} \mathrm{C}$, with a heating rate of $20^{\circ} \mathrm{C} \cdot \mathrm{min}^{-1}$. Pyris v.11 software was used for data analysis. The low-temperature DSC experiments were performed in a Perkin-Elmer Pyris 1 power compensation calorimeter. A liquid nitrogen Cryofill cooling unit was used, and helium at a $20 \mathrm{~mL} \cdot \mathrm{min}^{-1}$ flux was employed as the purge gas. Samples were cooled to $-170^{\circ} \mathrm{C}$ and then heated to room temperature at a $50^{\circ} \mathrm{C} \cdot \mathrm{min}^{-1}$ rate. The obtained data were analysed using TA Instruments Universal Analysis V4.1D software.

Acknowledgments - Financial support by the European Union LIFE+ Programme under project " $\mathrm{CO}_{2}$ Operation: Integrated agroforestry practices and nature conservation against climate change" (ref. LIFE11 ENV/ES/000535) is gratefully acknowledged. The authors would also like to thank the support of Fundação para a Ciência e a Tecnologia (FCT) under project UID/QUI/UI0313/2013 and the COMPETE Programme. 


\section{References}

[1] Gomes PB, Mata VG, Rodrigues AE. (2005) Characterization of the Portuguese-grown Cistus ladanifer essential oil. Journal of Essential Oil Research, 17, 160-165.

[2] Gülz P-G, Kobold U, Michaelis K, Vostrowsky O. (1984) The composition of terpene hydrocarbons in the essential oils from leaves of four Cistus species. Zeitschrift für Naturforschung C, 39, 699-704.

[3] Verdeguer M, Blázquez MA, Boira H. (2012) Chemical composition and herbicidal activity of the essential oil from a Cistus ladanifer L. population from Spain. Natural Product Research, 26, 1602-1609.

[4] Bessah R, Benyoussef E-H. (2014) Essential oil composition of Erica arborea L. leaves from Algeria. Journal of Essential Oil Bearing Plants, 17, 931-935.

[5] Khasan YY, Zorina AD, Matyukhina LG, Saltykova IA. (1977) Triterpenoids of some plants growing in Siberia. Chemistry of Natural Compounds, 13, 109-109.

[6] Hegnauer R, Hegnauer M. (1962) Chemotaxonomie der Pflanzen; eine Übersicht über die Verbreitung und die systematische Bedeutung der Pflanzenstoffe, Birkhäuser, Basel.

[7] De la Rosa JM, González-Pérez JA, González-Vázquez R, Knicker H, López-Capel E, Manning DAC, González-Vila FJ. (2008) Use of pyrolysis/GC-MS combined with thermal analysis to monitor $\mathrm{C}$ and $\mathrm{N}$ changes in soil organic matter from a Mediterranean fire affected forest. Catena, 74, 296-303.

[8] Cancellieri D, Leoni E, Rossi JL. (2005) Kinetics of the thermal degradation of Erica arborea by DSC: Hybrid kinetic method. Thermochimica Acta, 438, 41-50.

[9] Leroy V, Cancellieri D, Leoni E. (2006) Thermal degradation of ligno-cellulosic fuels: DSC and TGA studies. Thermochimica Acta, 451, 131-138.

[10] Fan M, Dai D, Huang B. (2012) Fourier transform infrared spectroscopy for natural fibres. In Fourier transform-materials analysis. Salih SM. (Ed.), InTech, Rijeka (Croatia), 45-68.

[11] Bodirlau R, Teaca CA, Spiridon I. (2009) Preparation and characterization of composites comprising modified hardwood and wood polymers/poly (vinyl chloride). BioResources, 4, 1285-1304.

[12] Vlachos N, Skopelitis Y, Psaroudaki M, Konstantinidou V, Chatzilazarou A, Tegou E. (2006) Applications of Fourier transform-infrared spectroscopy to edible oils. Analytica Chimica Acta, 573, 459-465.

[13] Moharam M, Abbas L. (2010) A study on the effect of microwave heating on the properties of edible oils using FTIR spectroscopy. African Journal of Microbiology Research, 4, 1921-1927.

[14] Jović O, Smolić T, Jurišić Z, Meić Z, Hrenar T. (2013) Chemometric analysis of Croatian extra virgin olive oils from central Dalmatia region. Croatica Chemica Acta, 86, 335-344.

[15] Martín-Ramos P, Martín-Gil J, Ramos-Sánchez MC, Navas-Gracia LM, Hernández-Navarro S, Martín-Gil FJ. (2016) Vibrational and thermal characterization of seeds, pulp, leaves and seed oil of Rosa rubiginosa L. Boletín de la Sociedad Argentina de Botánica, 51, 429439.

[16] Alencar JW, Alves PB, Craveiro AA. (1983) Pyrolysis of tropical vegetable oils. Journal of Agricultural and Food Chemistry, 31, 12681270 .

[17] Huck CW. (2015) Advances of infrared spectroscopy in natural product research. Phytochemistry Letters, 11, 384-393.

[18] Li Y, Fabiano-Tixier A-S, Chemat F. (2014) Essential oils: From conventional to green extraction. In Essential oils as reagents in Green Chemistry, Li Y, Fabiano-Tixier A.-S, Chemat F. (Eds.), Springer International Publishing, New York, NY, USA, 9-20.

[19] Llusia J, Peñuelas J. (2000) Seasonal patterns of terpene content and emission from seven Mediterranean woody species in field conditions. American Journal of Botany, 87, 133-140. 

5.4. Publicación \#4:

ON THE CRYSTALLINITY OF CELLULOSE MICROFIBERS FROM ALKALINE AND IONIC LIQUIDS TREATMENTS OF CISTUS LADANIDER AND ERICA ARBOREA (enviada a BioResources, ms. ID 1234)

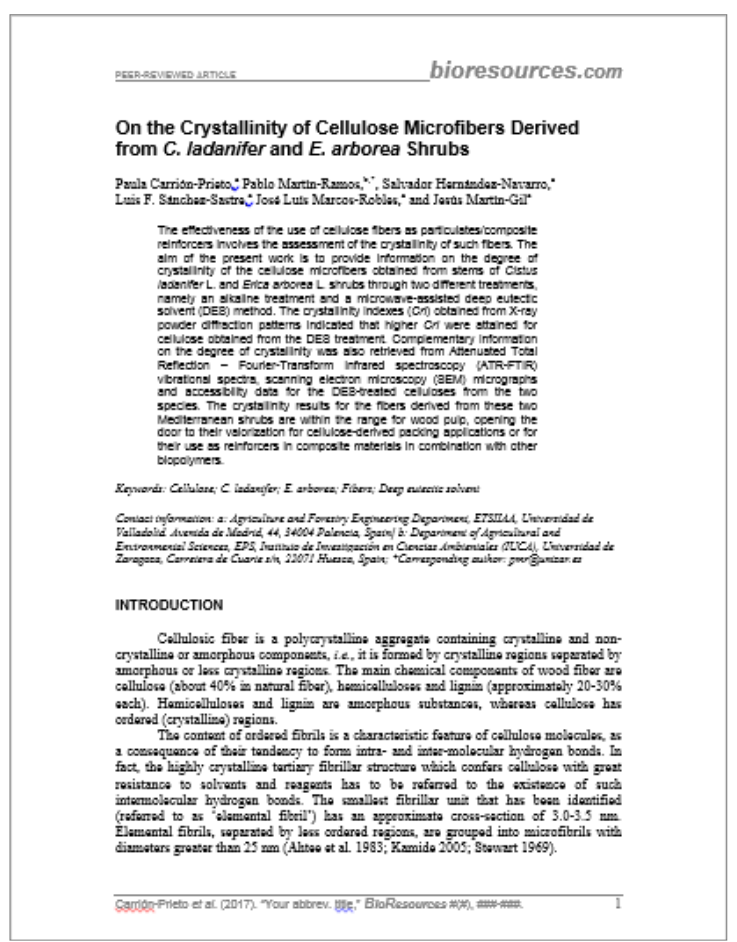





\title{
On the Crystallinity of Cellulose Microfibers Derived from C. ladanifer and E. arborea Shrubs
}

\author{
Paula Carrión-Prieto, ${ }^{\text {a }}$ Pablo Martín-Ramos,,${ }^{\text {, }}$, , Salvador Hernández-Navarro, ${ }^{\mathrm{a}}$

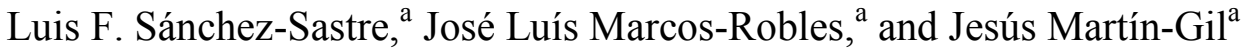

The effectiveness of the use of cellulose fibers as particulates/composite reinforcers involves the assessment of the crystallinity of such fibers. The aim of the present work is to provide information on the degree of crystallinity of the cellulose microfibers obtained from stems of Cistus ladanifer L. and Erica arborea L. shrubs through two different treatments, namely an alkaline treatment and a microwave-assisted deep eutectic solvent (DES) method. The crystallinity indexes $(\mathrm{Cr} I)$ obtained from X-ray powder diffraction patterns indicated that higher $\mathrm{CrI}$ were attained for cellulose obtained from the DES treatment. Complementary information on the degree of crystallinity was also retrieved from Attenuated Total Reflection - Fourier-Transform Infrared spectroscopy (ATR-FTIR) vibrational spectra, scanning electron microscopy (SEM) micrographs and accessibility data for the DES-treated celluloses from the two species. The crystallinity results for the fibers derived from these two Mediterranean shrubs are within the range for wood pulp, opening the door to their valorization for cellulose-derived packing applications or for their use as reinforcers in composite materials in combination with other biopolymers.

Keywords: Cellulose; C. ladanifer; E. arborea; Fibers; Deep eutectic solvent

Contact information: a: Agriculture and Forestry Engineering Department, ETSIIAA, Universidad de Valladolid. Avenida de Madrid, 44, 34004 Palencia, Spain; b: Department of Agricultural and Environmental Sciences, EPS, Instituto de Investigación en Ciencias Ambientales (IUCA), Universidad de Zaragoza, Carretera de Cuarte s/n, 22071 Huesca, Spain; *Corresponding author: pmr@unizar.es

\section{INTRODUCTION}

Cellulosic fiber is a polycrystalline aggregate containing crystalline and noncrystalline or amorphous components, i.e., it is formed by crystalline regions separated by amorphous or less crystalline regions. The main chemical components of wood fiber are cellulose (about $40 \%$ in natural fiber), hemicelluloses and lignin (approximately 20$30 \%$ each). Hemicelluloses and lignin are amorphous substances, whereas cellulose has ordered (crystalline) regions.

The content of ordered fibrils is a characteristic feature of cellulose molecules, as a consequence of their tendency to form intra- and inter-molecular hydrogen bonds. In fact, the highly crystalline tertiary fibrillar structure which confers cellulose with great resistance to solvents and reagents has to be referred to the existence of such intermolecular hydrogen bonds. The smallest fibrillar unit that has been identified (referred to as 'elemental fibril') has an approximate cross-section of 3.0-3.5 nm. Elemental fibrils, separated by less ordered regions, are grouped into microfibrils with diameters greater than 25 nm (Ahtee et al. 1983; Kamide 2005; Stewart 1969). 
Natural plant fibers or biofibers have been used as a reinforcing material for different types of matrices. Attention has recently focused on their use for the reinforcement of natural polymer matrices and to attain environmentally-friendly composite materials (since they are produced from natural resources). The advantages of biofibers over traditional reinforcing fibers (such as fiberglass) are their low cost, low density (with good specific properties), low roughness, biodegradability and the need for less processing equipment. Some of the most widely used natural fibers for the obtaining of reinforced materials are sisal, jute, flax and cellulose fibers from different origins (Bledzki and Gassan 1999; Dante et al. 2014).

It is known that while Pinus laricio is the species with the highest relative content in lignin and Cistus ladanifer L. (gum rockrose) is one of the richest in cellulose (55-60\%), Erica arborea L. (tree heath) is the plant species with highest holocellulose (cellulose and hemicellulose) content (Barboni et al. 2010). These latter two species are evergreen small diameter hardwood shrubs and constitute a significant proportion of Mediterranean forest vegetation. Since 2012, field studies aimed at their valorization, funded by the European Union through the LIFE+ and Joule programs, have been conducted in several municipalities in the province of Zamora (Spain).

With a view to their valorization in cellulose-derived packing, a research topic which was included in the top 10 innovations for 2025 according to the Intellectual Property \& Science business of Thomson Reuters (Thomson Reuters 2014), an assessment of the crystallinity of their cellulose fibers is required. Consequently, the study presented herein aims to gain insight -by using different characterization techniques- on the degree of crystallinity of the cellulose microfibers obtained from the stems of the two aforementioned pyrophytes, upon application of two different treatments (viz. an alkaline method and a microwave-assisted deep eutectic solvent method).

\section{EXPERIMENTAL}

\section{Samples and Reagents}

The samples used in this study were collected from a plot located in the municipality of Ayoó de Vidriales $\left(42^{\circ} 07^{\prime} 10^{\prime \prime} \mathrm{N}, 6^{\circ} 06^{\prime} 59^{\prime \prime} \mathrm{W}\right)$, in the province of Zamora, Castilla y Leon, Spain. The chosen area $(>1.2$ ha) is a mixed shrubland in which the dominant shrub species are E. arborea and C. ladanifer (Carrión-Prieto et al. 2017).

Choline chloride, urea, sodium hydroxide and sodium thiosulfate were purchased from PanReac AppliChem (Barcelona, Spain). Titanium dioxide $\left(\mathrm{TiO}_{2}\right.$, anatase variety) and Avicel ${ }^{\circledR}$ were supplied by Sigma Aldrich Química SL (Madrid, Spain).

\section{Treatments}

Samples of the stem of C. ladanifer and E. arborea $(200 \mathrm{mg})$ were ground in a blade mill and predried at $70{ }^{\circ} \mathrm{C}$ for $24 \mathrm{~h}$ and then immersed in $4 \mathrm{~mL}$ of a $\mathrm{NaOH}$ solution $(25 \% \mathrm{w} / \mathrm{v})$ with vigorous stirring at ambient for $24 \mathrm{~h}$. The alkaline-treated fibers were subsequently washed with running tap water and then distilled water until no alkali was present in the wash, and finally dried under vacuum to a constant weight. The alkali wash was recycled to avoid environmental pollution.

Alternatively, a deep eutectic solvent (DES) ionic liquids system, based on choline chloride-urea, was also used for fibrillation of C. ladanifer and E. arborea wood 
cellulose. Choline chloride/urea DES was prepared by stirring the mixture of choline chloride and urea (mole ratio 1:2) at $80{ }^{\circ} \mathrm{C}$ until a homogeneous colorless liquid was formed (Sun et al. 2003) and then stored in a vacuum dryer. Subsequently, cellulose samples $(200 \mathrm{mg})$ were treated by a mixture of choline chloride/urea $+\mathrm{HNO}_{3}(1.18 \mathrm{~mL})$ with $\mathrm{TiO}_{2}(20 \mathrm{mg})$ as a catalyst, using a microwave digestion system -a Milestone (Sorisole, BG, Italy) Ethos-One microwave oven equipped with a magnetic stirrer system- at $120{ }^{\circ} \mathrm{C}$ for an effective time of $30 \mathrm{~min}$. Samples were allowed to cool down to room temperature. Then, the DES was removed by washing with water (da Silva Lacerda et al. 2015; Li et al. 2011; Zhang and Zhao 2011).

\section{Characterization}

For the determination of crystallinity via X-ray powder diffraction (XRPD), data were obtained with a Bruker (Billerica, MA, USA) D8 Advance Bragg-Brentano diffractometer, in reflection geometry, employing $\mathrm{Cu} \mathrm{K \alpha}$ radiation $(\lambda=1.5406 \AA)$. Diffractograms were collected by continuous scanning over a diffraction angle $2 \theta=5-$ $80^{\circ}$. The crystallinity index $(C r I)$ was calculated using equation $C r I=\left[1-I_{\mathrm{am}} / I_{002}\right] \times 100$ from an internal reference method by Segal et al. (1959). In this equation, $I_{002}$ is the maximum intensity of crystalline scatter at the 002 reflection (in the case of cellulose, at $2 \theta=22.3^{\circ}$ ) and $I_{\mathrm{am}}$ corresponds to the intensity of the minimum or 'valley' between the peaks at $2 \theta=18^{\circ}$ and $22^{\circ}$. The amorphicity index $(A m I)$ is, obviously, $A m I=1-C r I$.

The vibrational spectra of the materials in the $400-4000 \mathrm{~cm}^{-1}$ range were measured using a Thermo Scientific (Waltham, MA, USA) Nicolet iS50 FT-IR Spectrometer, equipped with an in-built diamond attenuated total reflection (ATR) system. The absorption bands at 1430 and $890 \mathrm{~cm}^{-1}$ were used for the calculation of the lateral order index (LOI) (Kljun et al. 2011; O'Connor et al. 1958).

SEM micrographs were obtained with an FEI (Hillsboro, OR, USA) Quanta 200FEG microscope with BSE and SE detectors, equipped with a Genesis energydispersive X-ray (EDS) spectrometer system. Non-metalized samples were used.

Iodine absorption measurements were used to test the accessibility of cellulose, according to ASTM D4607-94 method. The $I S V$ or iodine adsorption value (mg) was calculated as $I S V=[(a-b) \times c \times 254] / w$, where $a$ is the consumable sodium hyposulphite solution with control $(\mathrm{mL}), b$ is the consumable sodium hyposulphite solution with samples $(\mathrm{mL}), c$ is the molar concentration of sodium hyposulphite solution $(\mathrm{mol} / \mathrm{L})$, and $w$ is the dry weight of the sample $(\mathrm{g})$.

\section{RESULTS}

\section{Degree of Crystallinity from X-Ray Powder Diffraction Data}

The determination of the crystallinity indexes from the intensities of the $I_{002}$ and $I_{\mathrm{am}}$ peaks in the diffractograms of the cellulose fibers from E. arborea and C. ladanifer after alkaline treatment (Fig. 1.) resulted in $\mathrm{CrI}$ values of around $39 \%$ and $38 \%$, respectively.

For the cellulose fibers from E. arborea and $C$. ladanifer subjected to the microwave-assisted DES process (Fig. 2.), the calculation of the $\mathrm{CrI}$ yielded values of $51.2 \%$ and $51.6 \%$, respectively. 


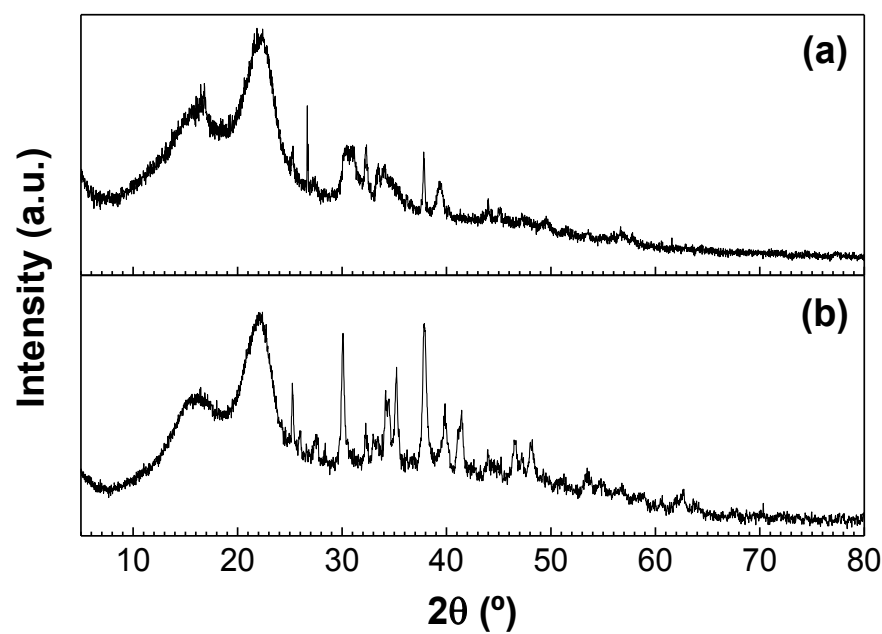

Fig. 1. X-ray powder diffraction patterns for the cellulose fibers from (a) E. arborea and (b) C. ladanifer after alkaline treatment.

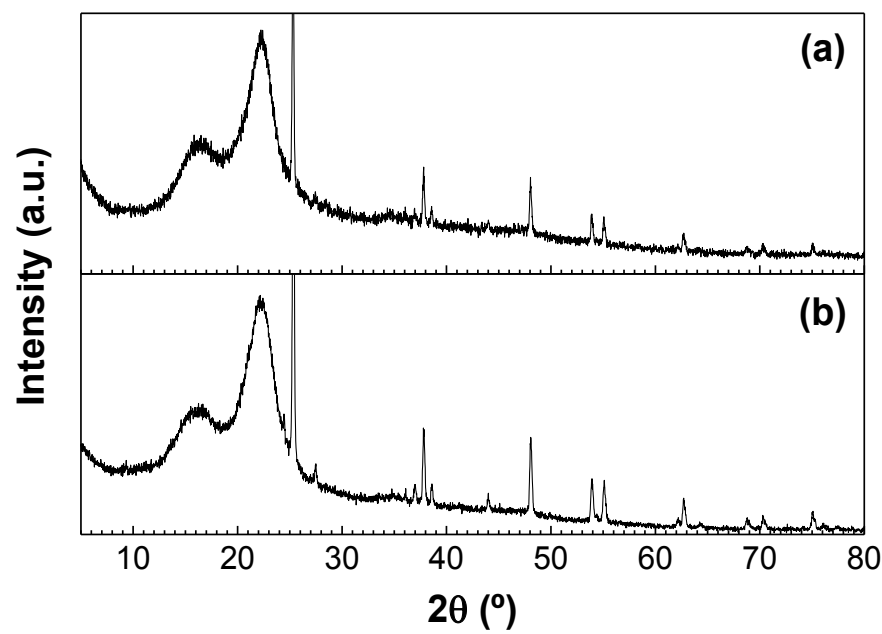

Fig. 2. X-ray powder diffraction patterns for the cellulose fibers from $(a)$ E. arborea and (b) C. ladanifer after microwave-assisted DES treatment for $30 \mathrm{~min}$. The very sharp peaks observed in both diffractograms for $2 \theta>25^{\circ}$ are spurious and can be unequivocally ascribed to some remaining $\mathrm{TiO}_{2}$, anatase variety (ICDD PDF \# 04-002-8296), used in the treatment.

\section{Degree of Crystallinity from ATR-FTIR and SEM Data}

Having observed a higher $\mathrm{CrI}$ with the microwave-assisted DES method, the crystallinity of the DES-treated samples was assessed using alternative approaches. The comparison was made both by examining the bands of the infrared spectra of the samples (calculating the lateral order index) as well as from information derived from SEM micrographs.

Firstly, the FTIR spectra (Fig. 3.) allowed to retrieve information on the interferences responsible for the uncertainty which affected the CrI determinations from the X-ray powder diffraction patterns: the observation of minimized peaks at $1730 \mathrm{~cm}^{-1}$ (associated with $\mathrm{C}=\mathrm{O}$ bonds of non-conjugated ketones present in hemicellulose (Afanas' ev et al. 2007; Morán et al. 2008; Pandey 1999)) evinced that the obtained samples had a low contamination of amorphous hemicellulose. However, the contamination with lignin would be higher: in fact, bands of medium intensity at 1268 and $1378 \mathrm{~cm}^{-1}$ attributable to guaiacyl and syringyl units from lignin could be observed. 


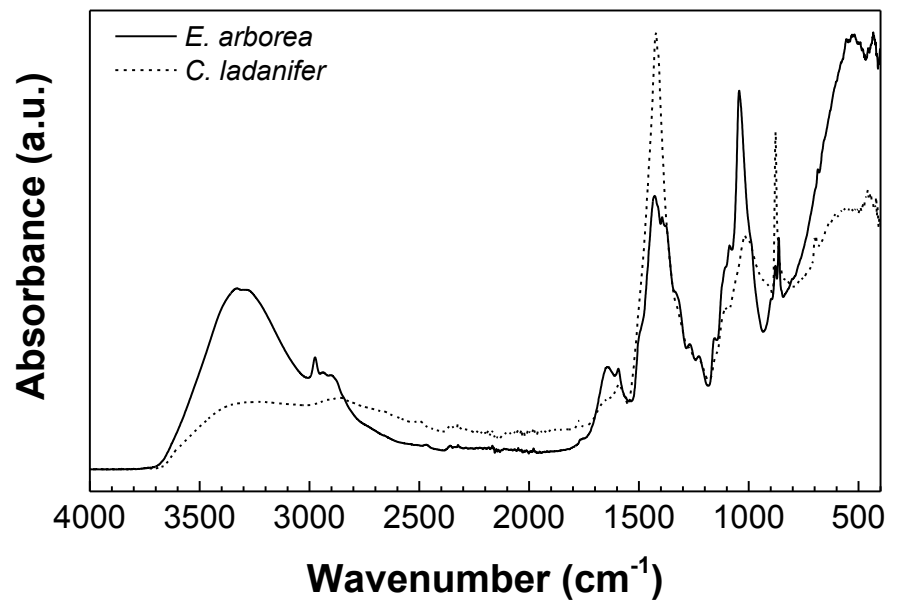

Fig. 3. ATR-FTIR spectra of the cellulose fibers from E. arborea (solid line) and C. ladanifer (dashed line) after microwave-assisted DES treatment of the inner stem.

In relation to the crystallinity, the lateral order index ( $L O I)$ based on the ratio of absorption bands at specific wavenumbers can be used to interpret qualitative changes in cellulose crystallinity: generally, as the $L O I$ decreases crystallinity also decreases (O'Connor et al. 1958). The absorption bands at 1430 and $893 \mathrm{~cm}^{-1}$ can be used to study the type of crystalline cellulose and the crystallinity changes (Kljun et al. 2011; Oh et al. 2005) because crystalline cellulose I spectrum clearly differs in this band from those of cellulose II and amorphous cellulose. From the intensities of the bands in the spectra, the $L O I$ values for $C$. ladanifer and E. arborea samples were 2.2 and 2.9, respectively, suggesting a higher crystallinity for the E. arborea-derived sample than for the treated cellulose from C. ladanifer.

On the other hand, and also according Kljun et al. (2011), if a cellulose fiber has a significant amount of crystalline cellulose I, the absorption band shifts toward 1430 $\mathrm{cm}^{-1}$ and the amounts of cellulose II and amorphous cellulose decrease. Because of a closer proximity of such band to $1430 \mathrm{~cm}^{-1}$ for E. arborea $\left(1428 \mathrm{~cm}^{-1}\right)$ than for $C$. ladanifer $\left(1422 \mathrm{~cm}^{-1}\right)$, a higher crystallinity can be presumed for the cellulose obtained from the former.

Valuable information, complementary to previous results, could also be obtained from the SEM micrographs of both samples. In the micrographs obtained for the fibers of C. ladanifer inner stem (Fig. 4.a and Fig. 4.b), a structure made up of cellulose fibrils agglomerated with each other, due to an incomplete removal of lignin, could be observed. On the other hand, E. arborea cellulose fibers (Fig. 4.c and Fig. 4.d) showed a more organized and regular morphology (more oriented) than those of $C$. ladanifer. From the micrographs obtained for the fibers of the inner stem of E. arborea it was also possible to recognize their dimensions: cylinders of length ranging from 100 to $110 \mu \mathrm{m}$ and with diameters between 9.20 and $15.30 \mu \mathrm{m}$. The aspect ratio $(\mathrm{L} / \mathrm{d}=7-10)$ of these microfibers is high compared to that of untreated fibers (1.9-3.3) and makes them very suitable for sonication to obtain nanowhiskers, an application contemplated in the manufacture of composites. 

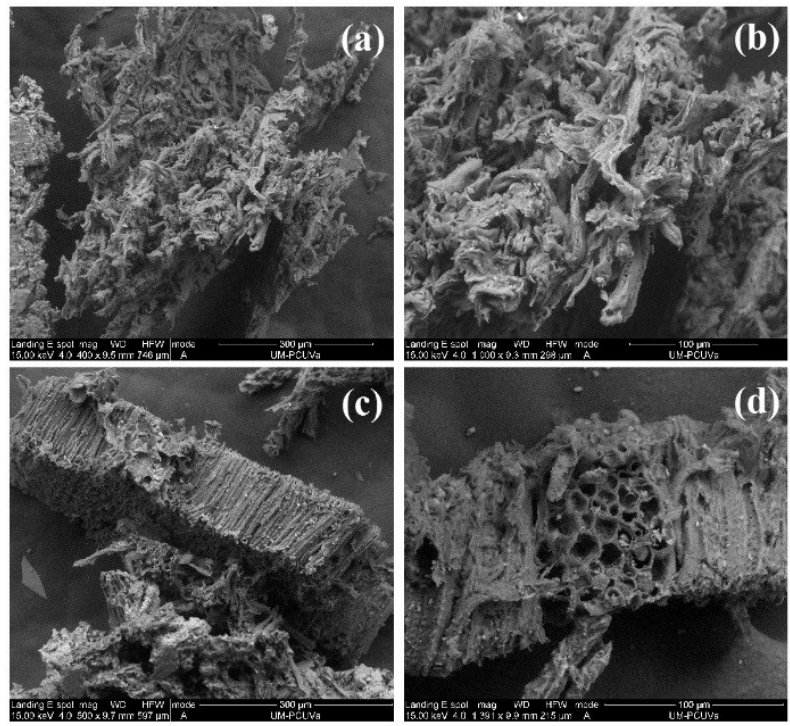

Fig. 4. SEM micrographs of the fibers after microwave-assisted DES-based treatment: inner stem of $C$. ladanifer [400× $(a)$ and $1000 \times(b)$ magnification] and inner stem of E. arborea [500× $(c)$ and $1400 \times(d)$ magnification].

\section{Crystallinity from Accessibility Data}

The accessibility of cellulose concerns the degree of difficulty with which some low-molecular mass reagents are able to reach the hydroxyl groups of cellulose. Adsorption of various solutes onto fibers occurs mainly on the amorphous regions and usually to a lesser degree on the surfaces of crystallized areas. Thus, any method to measure the fiber accessibility can be used for estimation of crystallinity (Roberts 1991; Schleicher et al. 1991). The accessibility of DES-treated cellulose fibers from $C$. ladanifer and E. arborea was studied in comparison with Avicel ${ }^{\circledR}$ microcrystalline cellulose by means of iodine sorption method and results are summarized in Table 11. Accessibility percentages were estimated taking the accessibility of amorphous cellulose (ISV of $412 \mathrm{mg} \mathrm{I}_{2} / \mathrm{g}$ ) as $100 \%$.

Table 1. ISV Values and Accessibility and Crystallinity Estimations for Treated Cellulose Fibers from Different Origins

\begin{tabular}{|c|c|c|c|}
\hline & lodine sorption value $\left(\mathrm{mgl}_{2} / \mathrm{g}\right.$ cellulose) & Accessibility (\%) & Crystallinity (\%) \\
\hline C. ladanifer & 103.5 & 25.1 & 74.9 \\
\hline E. arborea & 113.9 & 27.6 & 72.4 \\
\hline Avicel $^{(B)}$ & 78.3 & 19.0 & 81.0 \\
\hline
\end{tabular}

This indirect method led to significantly higher crystallinity estimations than the other methods discussed above. Further, the value for $C$. ladanifer-derived cellulose would be higher than that from E. arborea, and both of them would be quite close to that obtained for Avicel ${ }^{\circledR}$ reference material.

\section{DISCUSSION}

The crystallinity indices obtained from the X-ray powder diffractograms using the method proposed by Segal et al. (1959), suggested a higher effectiveness of the microwave-assisted DES treatment in comparison with the application of the alkaline treatment. Although this method is widely accepted for comparing the relative differences between samples, the obtained $\mathrm{CrI}$ values can only be taken as a rough 
approximation, since a simple height comparison cannot be expected to provide a reasonable estimate of cellulose crystallinity, as it excludes contributions from the other crystalline peaks and neglects variation in peak width, which can also be affected by crystallite size (Evans et al. 1995; French and Santiago Cintrón 2012; Ju et al. 2015; Park et al. 2010). It should be remembered that Segal et al. (1959) only intended this method to be used as a 'time-saving empirical measure of relative crystallinity'. The more accurate XRD deconvolution method could not be used for the samples studied herein, since the four/five peak fitting procedures are only reliable for purer cellulose samples (e.g., filter paper, bleached kraft pulp, neutral sulfite semi-chemical pulp, etc. (Garvey et al. 2005; Hult and Berglund 2003)), which result in very clean diffraction patterns.

As regards the results from the other indirect measurement techniques for DEStreated samples, the degree of crystallinity was more favorable to $C$. ladanifer than to $E$. arborea or vice versa depending on the chosen measurement method. Whereas the ATR-FTIR vibrational spectra and SEM micrographs suggested higher crystallinity values for $E$. arborea than for $C$. ladanifer, the indirect accessibility data from $I S V$ estimate pointed at a higher degree of crystallinity for $C$. ladanifer. In any case, the differences were not significant.

The percentage of crystallinity for cellulose is a determining characteristic in terms of its possible applications. For example, the low crystallinity of cellulose from pineapple peel has been invoked to explain the excellent results obtained in the preparation of solutions of that material in polyethylene glycol and in the manufacturing of polyurethane foams (Vega-Baudrit et al. 2007). In contrast, the high crystallinity of the cellulose from ramie fibers $(C r I=0.89)$ or Avicel $^{\circledR}(C r I=0.81-0.83)$ (Moya Portuguéz et al. 1992) lends itself to the reinforcement for plastics which could be used in place of glass (Mathijsen 2016).

In the case of the wood from the stems of $C$. ladanifer and E. arborea, the crystallinity of their celluloses, in the $52-75 \%$ range, would be within the interval established for wood pulp (60-85\%) (Núñez 2008). Thus, it can be anticipated that these treated cellulose fibers would be suitable for their application as reinforcers of composites, in combination with natural polymers, such as chitosan (Akhlaghi et al. 2013; de Mesquita et al. 2010), natural rubber (Bendahou et al. 2009), or soy protein (Wang et al. 2006).

\section{CONCLUSIONS}

1. The information resulting from XRD data allowed to establish that the degree of crystallinity of the MW-assisted DES-treated samples was higher than that of their alkaline-treated counterparts.

2. The complementary ATR-FTIR spectra, SEM images and indirect accessibility data from $I S V$ for the DES-treated samples suggested that there were no significant differences in the crystallinity of the cellulose microfibers derived from these two Mediterranean shrubs.

3. The results of the present study indicate that the crystallinity of the treated fibers of both $C$. ladanifer and $E$. arborea would be are within the range established for wood pulp, which makes them suitable to be used in combination with natural polymers for the manufacturing of composite materials. 


\section{ACKNOWLEDGMENTS}

This work was funded by the European Union LIFE+ Programme, under project $" \mathrm{CO}_{2}$ Operation: Integrated agroforestry practices and nature conservation against climate change" (ref. LIFE11 ENV/ES/000535). Access to TAIL-UC facility, funded under QREN-Mais Centro Project ICT_2009_02_012_1890, is also gratefully acknowledged. The authors would also like to thank Prof. P.M. Matei and Prof. F. Garrido-Laurnaga for their collaboration.

\section{REFERENCES CITED}

Afanas' ev, N., Prokshin, G., Lichutina, T., Gusakova, M., Vishnyakova, A., Sukhov, D., and Derkacheva, O. Y. (2007). "Effect of residual lignin on the supramolecular structure of sulfate hardwood cellulose: a Fourier IR study." Russian Journal of Applied Chemistry, 80(10), 1724-1727. DOI: 10.1134/S1070427207100254.

Ahtee, M., Hattula, T., Mangs, J., and Paakkari, T. (1983). "An X-ray diffraction method for determination of crystallinity in wood pulp." Paperi Ja Puu, 65(8), 475-480. DOI:

Akhlaghi, S. P., Berry, R. C., and Tam, K. C. (2013). "Surface modification of cellulose nanocrystal with chitosan oligosaccharide for drug delivery applications." Cellulose, 20(4), 1747-1764. DOI: 10.1007/s10570-013-9954-y.

Barboni, T., Pellizzaro, G., Arca, B., Chiaramonti, N., and Duce, P. (2010). "Analysis and origins of volatile organic compounds smoke from ligno-cellulosic fuels." Journal of Analytical and Applied Pyrolysis, 89(1), 60-65. DOI: 10.1016/j.jaap.2010.05.006.

Bendahou, A., Habibi, Y., Kaddami, H., and Dufresne, A. (2009). "Physico-chemical characterization of palm from Phoenix dactylifera L, Preparation of cellulose whiskers and natural rubber-based nanocomposites." Journal of Biobased Materials and Bioenergy, 3(1), 81-90. DOI: 10.1166/jbmb.2009.1011.

Bledzki, A., and Gassan, J. (1999). "Composites reinforced with cellulose based fibres." Progress in Polymer Science, 24(2), 221-274. DOI: 10.1016/s00796700(98)00018-5.

Carrión-Prieto, P., Hernández-Navarro, S., Martín-Ramos, P., Sánchez-Sastre, L. F., Garrido-Laurnaga, F., Marcos-Robles, J. L., and Martín-Gil, J. (2017). "Mediterranean shrublands as carbon sinks for climate change mitigation: new root-to-shoot ratios." Carbon Management, 1-11. DOI: 10.1080/17583004.2017.1285178.

da Silva Lacerda, V., López-Sotelo, J. B., Correa-Guimarães, A., Hernández-Navarro, S., Sánchez-Bascones, M., Navas-Gracia, L. M., Martín-Ramos, P., PérezLebeña, E., and Martín-Gil, J. (2015). "A kinetic study on microwave-assisted conversion of cellulose and lignocellulosic waste into hydroxymethylfurfural/furfural." Bioresource Technology, 180(0), 88-96. DOI: 10.1016/j.biortech.2014.12.089.

Dante, R. C., Sánchez-Arévalo, F. M., Huerta, L., Martín-Ramos, P., Navas-Gracia, L. M., and Martín-Gil, J. (2014). "Composite Fiber Based on Sisal Fiber and Calcium Carbonate." Journal of Natural Fibers, 11(2), 121-135. DOI: 10.1080/15440478.2013.849644. 
de Mesquita, J. P., Donnici, C. L., and Pereira, F. V. (2010). "Biobased nanocomposites from layer-by-layer assembly of cellulose nanowhiskers with chitosan." Biomacromolecules, 11(2), 473-480. DOI: 10.1021/bm9011985.

Evans, R., Newman, R. H., Roick, U. C., Suckling, I. D., and Wallis, A. F. A. (1995). "Changes in cellulose crystallinity during kraft pulping. Comparison of infrared, X-ray diffraction and solid state NMR results." Holzforschung, 49(6), 498-504. DOI: 10.1515/hfsg.1995.49.6.498.

French, A. D., and Santiago Cintrón, M. (2012). "Cellulose polymorphy, crystallite size, and the Segal Crystallinity Index." Cellulose, 20(1), 583-588. DOI: 10.1007/s10570-012-9833-y.

Garvey, C. J., Parker, I. H., and Simon, G. P. (2005). "On the Interpretation of X-Ray Diffraction Powder Patterns in Terms of the Nanostructure of Cellulose I Fibres." Macromolecular Chemistry and Physics, 206(15), 1568-1575. DOI: 10.1002/macp.200500008.

Hult, K., and Berglund, P. (2003). "Engineered enzymes for improved organic synthesis." Curr Opin Biotechnol, 14(4), 395-400. DOI: 10.1023/a:1024080700873.

Ju, X., Bowden, M., Brown, E. E., and Zhang, X. (2015). "An improved X-ray diffraction method for cellulose crystallinity measurement." Carbohydrate Polymers, 123, 476-481. DOI: 10.1016/j.carbpol.2014.12.071.

Kamide, K. (2005). Cellulose and cellulose derivatives: molecular characterization and its applications, Elsevier, Amsterdam.

Kljun, A., Benians, T. A. S., Goubet, F., Meulewaeter, F., Knox, J. P., and Blackburn, R. S. (2011). "Comparative analysis of crystallinity changes in cellulose I polymers using ATR-FTIR, X-ray diffraction, and carbohydrate-binding module probes." Biomacromolecules, 12(11), 4121-4126. DOI: 10.1021/bm201176m.

Li, C., Zhao, Z. K., Cai, H., Wang, A., and Zhang, T. (2011). "Microwave-promoted conversion of concentrated fructose into 5-hydroxymethylfurfural in ionic liquids in the absence of catalysts." Biomass and Bioenergy, 35(5), 2013-2017. DOI: 10.1016/j.biombioe.2011.01.055.

Mathijsen, D. (2016). "Cellulose as reinforcing material for plastics: an alternative between talcum and glass fiber." Reinforced Plastics, 60(3), 151-153. DOI: 10.1016/j.repl.2016.04.069.

Morán, J. I., Alvarez, V. A., Cyras, V. P., and Vázquez, A. (2008). "Extraction of cellulose and preparation of nanocellulose from sisal fibers." Cellulose, 15(1), 149-159. DOI: 10.1007/s10570-007-9145-9.

Moya Portuguéz, M. E., Durán, M., and Sibaja Ballesteros, M. R. (1992). "Obtención de lignina y celulosa de residuos de maíz." Uniciencia, 9(1), 45-50. DOI:

Núñez, C. E. (2008). "Química de la madera. Celulosa." Pulpa y Papel I, PROCYP, Facultad de Ciencias Exactas, Químicas y Naturales, Universidad Nacional de Misiones, Misiones, Argentina, 57-65.

O'Connor, R. T., DuPré, E. F., and Mitcham, D. (1958). "Applications of infrared absorption spectroscopy to investigations of cotton and modified cottons Part I: physical and crystalline modifications and oxidation." Textile Research Journal, 28(5), 382-392. DOI: 10.1177/004051755802800503.

Oh, S. Y., Yoo, D. I., Shin, Y., and Seo, G. (2005). "FTIR analysis of cellulose treated with sodium hydroxide and carbon dioxide." Carbohydrate Research, 340(3), 417-428. DOI: 10.1016/j.carres.2004.11.027.

Pandey, K. (1999). "A study of chemical structure of soft and hardwood and wood polymers by FTIR spectroscopy." Journal of Applied Polymer Science, 71(12), 
1969-1975. DOI: 10.1002/(SICI)1097-4628(19990321)71:12<1969::AIDAPP6>3.0.CO;2-D.

Park, S., Baker, J. O., Himmel, M. E., Parilla, P. A., and Johnson, D. K. (2010). "Cellulose crystallinity index: measurement techniques and their impact on interpreting cellulase performance." Biotechnology for Biofuels, 3(1), 10. DOI: 10.1186/1754-6834-3-10.

Roberts, G. A. F. (1991). "Accessibility of cellulose." Paper Chemistry, J. C. Roberts, ed., Springer Netherlands, Dordrecht, 9-24.

Schleicher, H., Kunze, J., and Lang, H. (1991). "Physico-chemical methods for the characterisation of cellulose reactivity." Wood Chemistry, 2, 38-41. DOI:

Segal, L., Creely, J., Martin, A., and Conrad, C. (1959). "An empirical method for estimating the degree of crystallinity of native cellulose using the X-ray diffractometer." Textile Research Journal 29(10), 786-794. DOI: 10.1177/004051755902901003.

Stewart, C. M. (1969). "The formation and chemical composition of hardwoods." Appita, 22(4), R32. DOI:

Sun, Y., Guo, T., Sui, Y., and Li, F. (2003). "Quantitative determination of rutin, quercetin, and adenosine in Flos Carthami by capillary electrophoresis." Journal of Separation Science, 26(12-13), 1203-1206. DOI: 10.1002/jssc.200301437.

Thomson Reuters. (2014). "The World in 2025: 10 predictions of innovation." Thomson Reuters IP \& Science, ScienceWatch.com.

Vega-Baudrit, J., Delgado-Montero, K., Sibaja-Ballestero, M., and Alvarado-Aguilar, P. (2007). "Uso alternativo de la melaza de la caña de azúcar residual para la síntesis de espuma rígidas de poliuretano (ERP) de uso industrial." Tecnología, Ciencia, Educación, 22(2), 101-107. DOI:

Wang, Y., Cao, X., and Zhang, L. (2006). "Effects of cellulose whiskers on properties of soy protein thermoplastics." Macromolecular Bioscience, 6(7), 524-531. DOI: 10.1002/mabi.200600034.

Zhang, Z., and Zhao, Z. (2011). "Production of 5-hydroxymethylfurfural from glucose catalyzed by hydroxyapatite supported chromium chloride." Bioresource Technology, 102(4), 3970-3972. DOI: 10.1016/j.biortech.2010.11.098. 
5.5. Publicación \#5:

FURFURAL, 5-HMF, ACID-SOLUBLE LIGNIN AND REDUCING SUGAR CONTENT IN BIOMASS OF CISTUS LADANIDER AND ERICA ARBOREA HYDROLYSATES BY UV-VIS SPECTROPHOTOMETRY 



\title{
Furfural, 5-HMF, acid-soluble lignin and non-reducing sugar contents in $\boldsymbol{C}$. ladanifer and $E$. arborea lignocellulosic biomass hydrolysates
}

\author{
Paula Carrión-Prieto ${ }^{1}$, Pablo Martín-Ramos ${ }^{2 *}$, Salvador Hernández-Navarro ${ }^{1}$, Jesús \\ Martín-Gil ${ }^{1}$
}

${ }^{1}$ Agriculture and Forestry Engineering Department, ETSIIAA, Universidad de Valladolid, Avda. Madrid 44, 34004, Palencia.

2 Department of Agricultural and Environmental Sciences, EPS, Instituto de Investigación en Ciencias Ambientales de Aragón (IUCA), Universidad de Zaragoza, Carretera de Cuarte s/n, 22071, Huesca. Phone: +34 (974) 292668; Fax: +34 (974) 239302; E-mail: pmr@unizar.es

\begin{abstract}
Cistus ladanifer L. and Erica arborea L. biomass hydrolysate components, obtained from microwave-assisted treatments with choline chloride/urea - $\mathrm{HNO}_{3} 10 \%, \mathrm{~N}, \mathrm{~N}$ dimethylacetamide $/ \mathrm{NaHCO}_{3}$ and $\mathrm{N}, \mathrm{N}$-dimethylacetamide $/ \mathrm{CH}_{3} \mathrm{OK}$ as solvents, have been measured using a spectrophotometric method. Interference from acid-soluble lignin was alleviated by the use of absorbance difference spectrum before and after reduction with $\mathrm{NaBH}_{4}$. The concentrations of furfural and 5-(hydroxymethyl)furfural in the filtrate were determined by measuring the absorbance of the difference spectrum at $277 \mathrm{~nm}$ and $285 \mathrm{~nm}$, respectively. Acid-soluble lignin was estimated by the absorbance of the reduced hydrolysate at $280 \mathrm{~nm}$. The total sugars content was calculated applying the Dubois et al. method at $490 \mathrm{~nm}$, while for reducing sugars the Miller et al. method at $540 \mathrm{~nm}$ was chosen. Non-reducing sugars content was calculated by difference between total sugar and reducing sugars percentages. Our results reports the suitability of applying MW-assisted choline chloride/urea deep eutectic solvent for extracting lignin, furfural, 5-HMF and sugars from $C$. ladanifer and $E$. arborea biomass. The best yield for lignin, furfural and 5-HMF was obtained for the 60 min-treatment; and the greatest increase in the production of total and non-reducing sugars occurred for the MW-treatment between 10 and 20 min.
\end{abstract}

\section{Introduction}

Lignin, interlaced with cellulose and hemicellulose, forms a complex crystal structure called lignocellulose that provides support and protection to plant cells [1]. This matrix is difficult to degrade, requiring treatments that break down its structure, hydrolyze the hemicellulose and increase the exposed surface to favor the enzymatic hydrolysis of cellulose [2]. For this purpose, different pretreatments can be used: physical processes, such as grinding or heating; chemical approaches, such as the addition of acids or bases; physical-chemical treatments, such as self-hydrolysis or thermo-hydrolysis; and biological ones, such as the use of enzymes capable of degrading lignin (ligninases). In the study presented herein, after a milling step, several types of treatments with different reagents/solvents have been assessed: based on an alkali $(\mathrm{NaOH})$, on a deep eutectic solvent (DES) mixture of choline chloride:urea and $\mathrm{HNO}_{3} 10 \%$, on $\mathrm{N}, \mathrm{N}$-dimethylacetamide (DMAc) $/ \mathrm{NaHCO}_{3}$ and on $\mathrm{DMAc} / \mathrm{CH}_{3} \mathrm{OK}$, respectively. DES were used to replace ionic liquids (ILs), which are more expensive and toxic. 
During the conventional treatments of the biomass, the high temperatures reached in the processes influence the composition of the by-products generated. While heating improves enzymatic hydrolysis, it also has important drawbacks, such as the formation of compounds that have an inhibitory effect on ethanol producing organisms. An alternative to conventional heating is the application of microwave radiation [3-5], in which the direct contact between the product and the electromagnetic field generated by the microwaves results in a volumetric heating that causes an instantaneous temperature increase [6,7].

The composition of the liquid phase of the treatments includes organic acids (mainly acetic acid, formic acid and levulinic acid), furan derivatives (2-furfuraldehyde, furfural and 5-(hydroxymethyl)-2-furaldehyde), and phenolic compounds (mainly coumaric acid, syringaldehyde and vanillin) [8,9]. Under acidic conditions and especially at high temperature, furfural is readily produced from pentoses and 5-HMF is readily formed from hexoses. Because both furfural and 5-HMF are formed from carbohydrates, they interfere with the accuracy of sugar analysis of any biomass materials. Furthermore, both are harmful to the fermentation of sugars if the concentrations of these compounds exceed certain thresholds [10].

This paper presents some MW-assisted treatments in different solvents carried out to break the intrincate structure of two shrubs, C. ladanifer and E. arborea. Our main objective was to compare the efficiency of the $\mathrm{ChCl} /$ urea $-\mathrm{HNO}_{3} 10 \%$ DES system vs. DMAc-based systems (DMAc/ $\mathrm{NaHCO}_{3}$ and $\mathrm{DMAc} / \mathrm{CH}_{3} \mathrm{OK}$ ) in order to extract lignin, furfural, 5-HMF and sugars.

\section{Materials and methods}

\section{Materials}

Samples of biomass (mainly from the stem) of two species typical of Mediterranean scrublands, Erica arborea L. and Cistus ladanifer L., were selected, dried in a stove and crushed in a knife mill. Furfural (CAS No. 98-01-1), HMF (CAS No. 67-47-0), lignin (CAS No. 8068-05-1) and D-(+)-glucose analytical standards (CAS No. 50-99-7) were purchased from Sigma-Aldrich. The standard solutions were prepared with deionized water. Sodium borohydride (CAS No. 16940-66-2), 3-amino-5-nitrosalicylic acid (DNS, CAS No. 831-51-6), phenol (CAS No. 108-95-2), choline chloride (CAS No. 6748-1), urea (CAS No. 57-13-6), titanium dioxide (CAS No. 13463-67-7), N, $N^{\prime}$ dimethylacetamide (DMAC, CAS No. 127-19-5), sodium bicarbonate (CAS No. 14455-8), potassium methoxide (CAS No. 865-33-8), sodium hydroxide (CAS No. 131073-2) and potassium sodium tartrate (CAS No. 6381-59-5) were also supplied by Sigma Aldrich.

\section{Methods}

\section{Microwave-assisted deep eutectic solvent}

A deep eutectic solvent "ionic liquid" system, based on choline chloride-urea (Figure 1 ) was assessed for the hydrolysis of $C$. ladanifer and E. arborea biomass. Choline chloride/urea DES was prepared by stirring the mixture of choline chloride and urea (mole ratio 1:2) at $80{ }^{\circ} \mathrm{C}$ until a homogeneous colorless liquid was formed, which was then stored in a vacuum dryer. Subsequently, biomass samples $(200 \mathrm{mg})$ were treated by a mixture $(8 \mathrm{~mL})$ of choline chloride/urea and $\mathrm{HNO}_{3} 10 \%$, with $\mathrm{TiO}_{2}(20 \mathrm{mg})$ as a 
catalyst into a microwave digestion system -a Milestone (Sorisole, BG, Italy) EthosOne microwave oven equipped with a magnetic stirrer system- at $120{ }^{\circ} \mathrm{C}$ for an effective time between 1 and of $60 \mathrm{~min}$. Samples were allowed to cool down to room temperature. The DES was finally removed by washing with water.

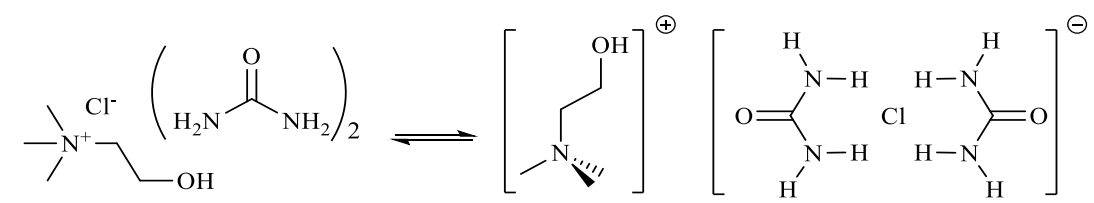

Figure 1. DES of $\mathrm{ChCl}$ and urea where a $[\text { choline }]^{+}$cation is energetically competitive with $\left[\mathrm{Cl}(\mathrm{urea})_{2}\right]^{-}$.

\section{Microwave-assisted DMAc-sodium bicarbonate polar aprotic solvent}

Alternatively to the DES-based method, $8 \mathrm{~mL}$ of a colourless, water-miscible, high boiling liquid -viz. $N, N^{\prime}$-dimethylacetamide (DMAc) with formula $\mathrm{CH}_{3} \mathrm{C}(\mathrm{O}) \mathrm{N}\left(\mathrm{CH}_{3}\right)_{2}-$ was used, in the presence of $40 \mathrm{mg}$ of sodium hydrogen carbonate $\left(\mathrm{NaHCO}_{3}\right)$, as a treatment agent for $C$. ladanifer and $E$. arborea woods (200 $\mathrm{mg}$ of biomass samples). The same procedure explained above for the microwave-assisted DES treatment was followed for the polar aprotic solvent-based treatment. DMAc was removed by washing with water.

\section{Microwave-assisted DMAc-potassium methoxide system}

The third approach hydrolytic treatment for $C$. ladanifer and E. arborea biomass (200 mg) was based on a mixture of $8 \mathrm{~mL}$ of DMAc with $40 \mathrm{mg}$ of potassium methoxide (commonly used as a catalyst for transesterification in the production of biodiesel). The methoxide ion was prepared in situ by reacting methanol with hydroxide. The solution was then treated as in the previously discussed methods.

\section{Alkaline treatment}

Solutions were prepared with $20 \mathrm{mg}$ of each sample and $2 \mathrm{~mL}$ of $0.1 \mathrm{M} \mathrm{NaOH}$, which were stirred for $24 \mathrm{~h}$. From these solutions, $0.3 \mathrm{~mL}$ of each sample were isolated and then diluted to $25 \mathrm{~mL}$ (to keep the concentration within the spectrophotometer measurement range and to avoid absorption flattening due to saturation). When necessary, $\mathrm{HCl}$ was used to preserve the $\mathrm{pH}$ neutral.

\section{ASL, Furfural and HMF content}

The quantification of soluble lignin based on its characteristic peaks at $205 \mathrm{~nm}$ and $280 \mathrm{~nm}$ has been questioned for the determination of soluble lignin because formic acid and acetic acid have significant absorption at $205 \mathrm{~nm}$ and furfural and HMF have absorption maxima close to 277 and $285 \mathrm{~nm}$, respectively. Recently, Sun et al. [11] reported a method to eliminate the interference of furanic compounds by reduction with alkaline sodium borohydride.

The acid-soluble lignin (ASL), furfural (F) and 5-(hydroxymethyl)-furfural (5-HMF) contents were determined according to the methodology proposed by Chi et al. [12], based on the measurement of their respective maximum absorbances at $205 \mathrm{~nm}, 277 \mathrm{~nm}$ and $285 \mathrm{~nm}$ and which makes use of the effect of the reduction with borohydride on the furfural and 5-HMF maxima. For these latter two chemical species, their initial absorbance in the UV-vis spectrum was measured and, after $30 \mathrm{mg}$ of sodium 
borohydride had been added (followed, after $5 \mathrm{~min}$, by the addition of a small amount of $\mathrm{HCl}$ ), absorbance measurements were repeated. Their associated absorption maxima at 277 and $285 \mathrm{~nm}$ completely disappeared upon reduction with $\mathrm{NaBH}_{4}$. Therefore, the furfural and HMF contents could be readily calculated from the absorbance difference before and after reduction $\left(\Delta A_{R}\right)$ at their respective wavelengths.

\section{Sugar content}

The quantification of reducing sugars was conducted according to Miller [13], using DNS as the most specific reagent in a solution containing sodium hydroxide and potassium sodium tartrate. The solution was prepared by mixing $0.8 \mathrm{~g}$ of $\mathrm{NaOH}, 15 \mathrm{~g}$ of sodium potassium tartrate and $0.5 \mathrm{~g}$ of DNS, completing up to $50 \mathrm{~mL}$ with distilled water. To ensure homogeneity, it was boiled for 5 minutes. It was then cooled with water and ice, $5 \mathrm{~mL}$ of water were added to compensate for the evaporated volume, and it was allowed to rest for 15 minutes. With this solution, that will be referred to as DNS, 1:1 mixtures were prepared with the samples and the standard solutions to be analyzed (usually $0.5 \mathrm{~mL}$ of DNS and $0.5 \mathrm{~mL}$ of either the sample or the standard solution). The determination of reducing sugars in these mixtures was conducted by measuring their absorbance at $540 \mathrm{~nm}$.

The total sugars determination was carried out in agreement with the method proposed by DuBois et al. [14]. This method is usually called 'phenol-sulfuric acid method' because in the preparation of the measuring solutions, 1-2 $\mathrm{mL}$ of sample, $1 \mathrm{~mL}$ of phenol $(5 \%)$ and $5 \mathrm{~mL}$ of concentrated sulfuric acid $(95.5 \%)$ are mixed in the test tubes. The test tubes containing these solutions were placed in a rack which was kept in a thermostatic bath, between 25 and $30{ }^{\circ} \mathrm{C}$, for $10-15$ min. Glucose at various concentrations was used as a standard. The spectrophotometric measurement of the total sugars was carried out at $490 \mathrm{~nm}$, that is, at the wavelength at which hexoses and their methylated derivatives exhibit their maximum absorption. Non-reducing sugars content was calculated by difference between the total sugars and the reducing sugars percentages.

\section{Calibration curves}

In order to obtain the calibration curves for each component under study, dissolutions with different concentrations of the analytical standards used as a reference (viz. furfural, lignin, 5-HMF and glucose) were prepared. Absorption values for increasing concentrations of the analytical standard were plotted and data was fitted with a straight line, in agreement with Beer's Law. The concentrations of furfural, 5-HMF and acidsoluble lignin were determined using these calibration curves. Each data point is the mean of three determinations. Standard deviation bars have been omitted for clarity.

\section{Results and discussions}

\section{Furfural, 5-HMF and ASL}

Calibration curves for ASL, furfural and 5-HMF are shown below (Figure 2). Excellent linear relationships (Eq. 1-3) were obtained at their three respective wavelengths (at $\lambda=280 \mathrm{~nm}$ for ASL, at $\lambda=277 \mathrm{~nm}$ for furfural and at $\lambda=285 \mathrm{~nm}$ for 5 HMF), with Pearson coefficients ( $R^{2}$ values) above 0.95 in all cases. 


$$
\begin{aligned}
& y_{A S L}=109.11 x+0.0841 ; R^{2}=0.9802 \\
& y_{F}=270.76 x+0.2236 ; R^{2}=0.9534 \\
& y_{H M F}=1056.2 x+0.017 ; R^{2}=0.9921
\end{aligned}
$$

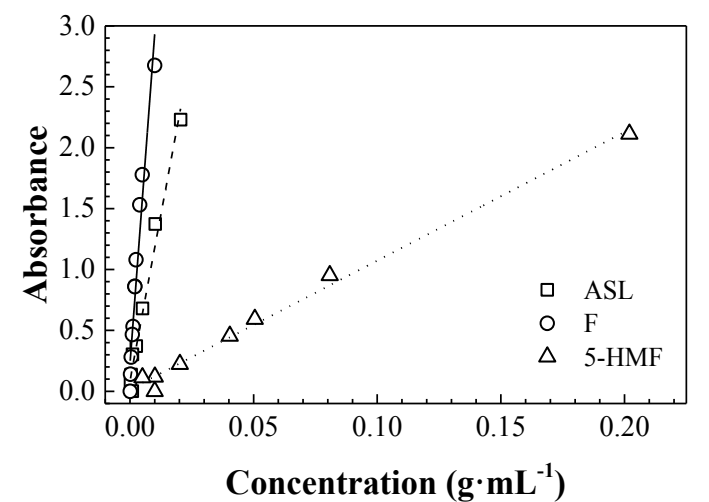

Figure 2. Calibration curves for furfural, acid-soluble lignin and 5-(hydroxymethyl)-furfural concentration.

The highest values of ASL, furfural and 5-HFM were generally obtained after $60 \mathrm{~min}$ of microwave-assisted "ionic liquid" treatment, both for E. arborea and C. ladaniferderived biomass (see Table 1). As noted above, furfural and 5-HMF values, obtained by the difference of the absorption values before and after the reduction with borohydride, were not influenced by the lignin content.

Table 1. Lignin, furfural and 5-HMF in hydrolysates after a deep eutectic solvent MW-assisted extraction.

\begin{tabular}{cccccccc}
\hline \multirow{2}{*}{ Treatment } & Time (min) & \multicolumn{3}{c}{ Erica arborea } & \multicolumn{3}{c}{ Cistus ladanifer } \\
& & Lignin (\%) & Furfural (\%) & HMF (\%) & Lignin & Furfural (\%) & HMF \\
$(\%)$
\end{tabular}


As depicted in Figure 3, both for E. arborea and $C$. ladanifer, the choline chloride/urea treatment was more effective in the production of ASL, furfural and 5HMF than the treatment with DMAc/sodium bicarbonate, which -in turn- showed a better performance than the DMAc/potassium methoxide approach.

It is worth noting that after the choline chloride/urea treatment, E. arborea samples produced higher content in furan-derived products than those of $C$. ladanifer, although the differences were not significant from a statistical point of view: it produced $0.22 \%$ more furfural and $0.07 \%$ more 5-HMF. However, treatment times below 10 min showed a higher production of furfural and 5-HMF from $C$. ladanifer than from E. arborea. As regards the lignin content in the liquid phase after the MW-assisted treatments, it was higher in E. arborea than in C. ladanifer.
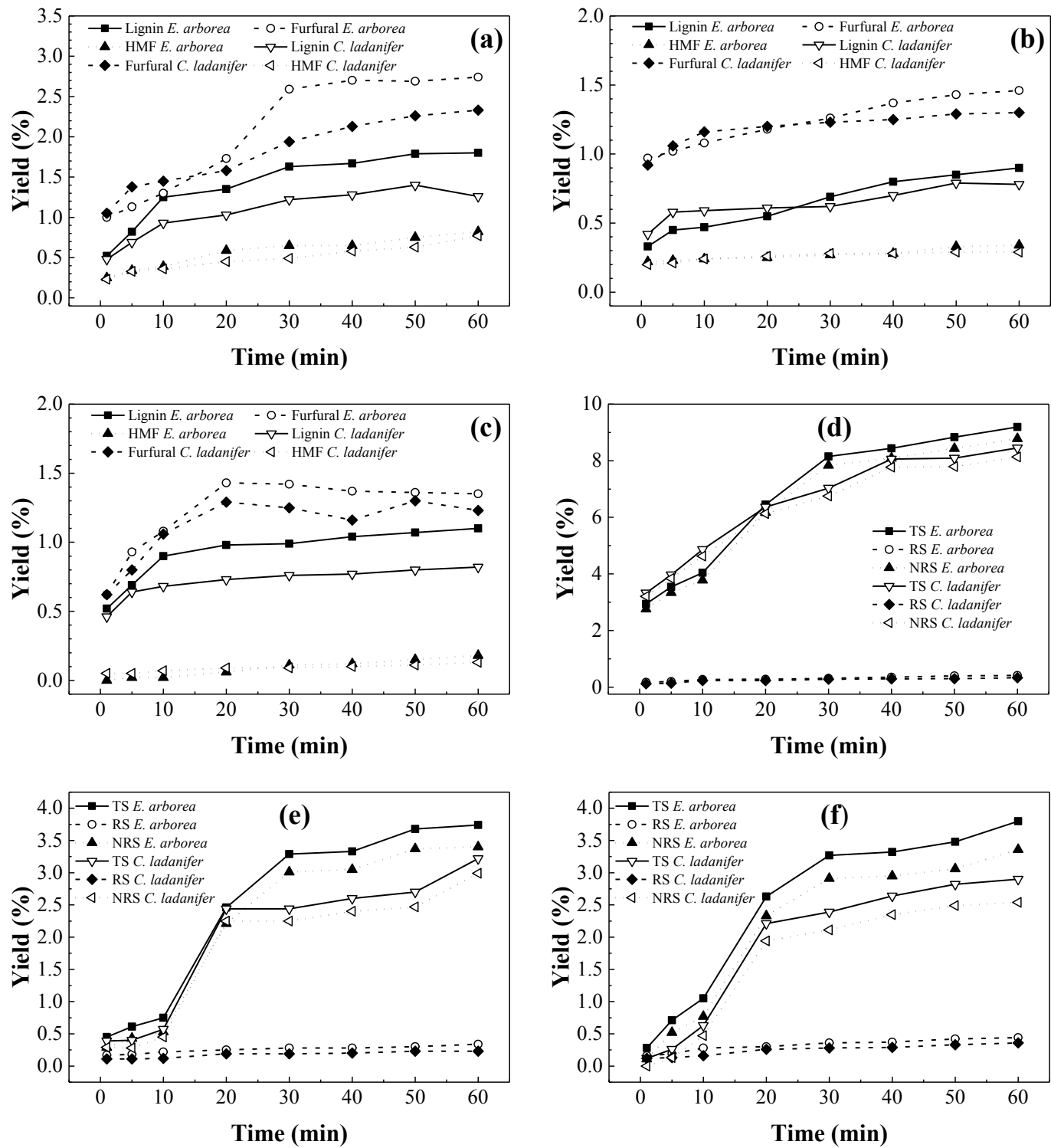

Figure 3. Lignin, furfural and HMF yields for the E. arborea and C. ladanifer lignocellulosic biomass hydrolysates after: $(a)$ a deep eutectic solvent MW-assisted extraction; $(b)$ a polar aprotic solvent MWassisted extraction; and (c) a DMAc/ $\mathrm{CH}_{3} \mathrm{OK}$ solvent $\mathrm{MW}$-assisted extraction. Total, reducing and no reducing sugars in the hydrolysates after: $(d)$ a $\mathrm{MW}$-assisted $\mathrm{ChCl} /$ urea treatment; $(e)$ a $\mathrm{MW}$-assisted $\mathrm{DMAc} / \mathrm{NaHCO}_{3}$ treatment; and $(f)$ a $\mathrm{MW}$-assisted $\mathrm{DMAc} / \mathrm{CH}_{3} \mathrm{OK}$ treatment. 
For the choline chloride/urea treated shrubs biomass, the values for soluble lignin content (1.26-1.80\%), furfural content (2.33-2.74\%) and 5-HMF content $(0.77-0.82 \%)$ were in agreement with those reported by Chi et al. [12] for the acid hydrolysis of Pinus taeda (ASL: 1.43\%, furfural: 2.02\%, and 5-HMF: 1.05\%). Da Silva et al. found furfural +5 -HMF contents ranging from $0.57 \%$ for macauba shell and up to $7.28 \%$ or native cellulose in one of its works [15], and furfural and 5-HMF values of 5.25\% and $0.87 \%$, respectively, for native cellulose in other of its investigations [16].

Non MW-assisted alkaline treatments (with $\mathrm{NaOH}$ ), used for comparison purposes, gave soluble lignin contents twice as high for E. arborea $(2.25 \%)$ as those for $C$. ladanifer $(1.31 \%)$, and both were higher than those obtained for the other treatments. However, furfural contents with the alkaline procedure were $0.40 \%$ for E. arborea and $0.19 \%$ for C. ladanifer, significantly lower than those obtained in the MW-assisted treatments. HMF contents $(0.52 \%$ and $0.47 \%$ for $E$. arborea and $C$. ladanifer, respectively) were similar to those obtained after $20 \mathrm{~min}$ of MW-assisted DES treatment and higher than those obtained in DMAc solvents.

Table 2. Comparative measurements of soluble lignin, furfural and HMF in the hydrolysates.

\begin{tabular}{|c|c|c|c|c|c|c|}
\hline \multirow{2}{*}{ Component } & \multirow{2}{*}{ Solvent } & \multicolumn{2}{|c|}{ Shrubs } & \multirow{2}{*}{$\begin{array}{c}\text { Native } \\
\text { cellulose }\end{array}$} & \multirow{2}{*}{ Hardwoods } & \multirow{2}{*}{ References } \\
\hline & & E. arborea & C. ladanifer & & & \\
\hline \multirow{4}{*}{ Lignin (\%) } & $\mathrm{ChCl} /$ urea & $0.52-1.80$ & $0.48-1.4$ & & & \\
\hline & $\mathrm{DMAc} / \mathrm{NaHCO}_{3}$ & $0.33-0.90$ & $0.42-0.79$ & & 1.43 & Chi et al [12] \\
\hline & $\mathrm{DMAc} / \mathrm{CH}_{3} \mathrm{OK}$ & $0.52-1.10$ & $0.46-0.82$ & & & \\
\hline & $\mathrm{NaOH}$ & 2.25 & 1.31 & & & \\
\hline \multirow{4}{*}{ Furfural (\%) } & $\mathrm{ChCl} /$ urea & $1.00-2.74$ & $1.05-2.33$ & $2.30-5.25$ & & Da Silva et al [15] \\
\hline & $\mathrm{DMAc} / \mathrm{NaHCO}_{3}$ & $0.97-1.46$ & $0.92-1-30$ & & & \\
\hline & $\mathrm{DMAc} / \mathrm{CH}_{3} \mathrm{OK}$ & $0.62-1.43$ & $0.62-1.30$ & & & \\
\hline & $\mathrm{NaOH}$ & 0.40 & 0.19 & & & \\
\hline \multirow{4}{*}{ HMF (\%) } & $\mathrm{ChCl} /$ urea & $0.25-0.82$ & $0.23-0.77$ & $0.23-0.87$ & & Da Silva et al [16] \\
\hline & $\mathrm{DMAc} / \mathrm{NaHCO}_{3}$ & $0.22-0.34$ & $0.20-0.29$ & & & \\
\hline & $\mathrm{DMAc} / \mathrm{CH}_{3} \mathrm{OK}$ & $0.00-0.18$ & $0.05-0.13$ & & & \\
\hline & $\mathrm{NaOH}$ & 0.52 & 0.47 & & & \\
\hline
\end{tabular}

\section{Sugar content}

In order to obtain the calibration curves for glucose (depicted in Figure 4), reference dissolutions with different concentrations of the analytical standard were prepared. The equation of the calibration curve of total sugars (Eq. 4) was built by applying the methodology proposed by DuBois et al. [14], measuring the absorbance at $490 \mathrm{~nm}$. On the other hand, the method by Miller [13] was used for the calibration for reducing sugars (Eq. 5), measuring the absorbance at $540 \mathrm{~nm}$. Eq. 6 is the difference resulting between the calibration curves of total and reducing sugars. $R^{2}$ values were close to 1 .

$$
\begin{array}{ll}
y_{T S}=5.0694 x+0.0525 ; R^{2}=0.9807 & \text { Eq. } 4 \\
y_{R S}=17.867 x+0.0442 ; R^{2}=0.9946 & \text { Eq. } 5 \\
y_{N S}=y_{490}-y_{540}=-12.7976 x+0.0083 & \text { Eq. } 6
\end{array}
$$




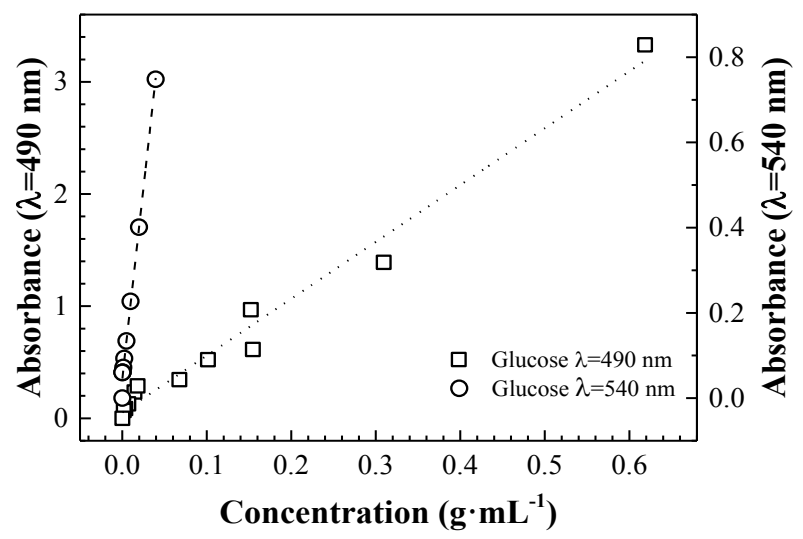

Figure 4. Calibration curves for glucose concentration.

From the data summarized in Table 3, it can be observed that the concentration of reducing sugars in the hydrolysates obtained from both species was low (0.23-0.44\%). Although $E$. arborea presented higher values than $C$. ladanifer, no statistically significant differences were observed. On the other hand, the production of nonreducing sugars was high, close to that of total sugars (provided that they were determined by subtracting the reducing sugars from the total ones).

It can also be observed that the greatest increase in the production of total sugars and therefore of non-reducing sugars- occurred for MW-treatment between 10 and 20 min, both for $C$. ladanifer and E. arborea. For reducing sugars this occurs only for DMAc treatments in the case of $C$. ladanifer. The greatest increase of production of reducing sugars for $E$. arborea was between 5 and $10 \mathrm{~min}$ for all the treatments.

Both for E. arborea and C. ladanifer hydrolysates, the treatment with ionic liquids was found to be more effective in terms of sugar production than the treatments based on DMAc. No significant differences were observed between the results of the microwave-assisted DMAc-potassium methoxide and the DMAc-sodium hydrogen carbonate systems.

Upon application of the choline chloride/urea treatment, E. arborea samples produced more total sugars than $C$. ladanifer ones. The reducing sugars content was similar, although slightly higher in the former.

Upon alkaline treatment (Table 4 ) by $24 \mathrm{~h}$, total sugar values obtained (4.63\% for $E$. arborea and $5.64 \%$ for $C$. ladanifer) were similar those obtained by 10-20 min MWassisted treatment in choline $\mathrm{ChCl} /$ urea solvent and higher than those in DMAc-based solvents. Reducing sugars production $(1.29 \%$ for E. arborea and $1.00 \%$ by C. ladanifer $)$ were three times higher than those of the microwave treatments. Non-reducing sugars for the $\mathrm{NaOH}$ treatment $(3.34 \%$ and $4.64 \%$, respectively) would be similar to those obtained for 5 min treatment with choline $\mathrm{ChCl} / \mathrm{urea}, 40-50 \mathrm{~min}$ with $\mathrm{DMAc} / \mathrm{NaHCO}_{3}$ and 50-60 min with DMAc/ $\mathrm{CH}_{3} \mathrm{OK}$ in the case of E. arborea, and a 10 min treatment with choline $\mathrm{ChCl} /$ urea in the case of $C$. ladanifer (the $4.64 \%$ was much higher than values resulting from the DMAc solvent treatments).

For comparison purposes, Table 4 shows the concentration of total sugars and reducing sugars for corncob (twice higher) and bamboo (ten times higher) [17] 
Table 3. Total (TS), reducing (RS) and non-reducing (NRS) sugars concentrations for the MW-assisted $\mathrm{ChCl} /$ urea $\mathrm{DMAc} / \mathrm{NaHCO}_{3}$ and $\mathrm{DMAc} / \mathrm{CH}_{3} \mathrm{OK}$ treatments as a function of exposure times.

\begin{tabular}{cccccccc}
\hline \multirow{2}{*}{ Treatment } & \multirow{2}{*}{ Time (min) } & \multicolumn{3}{c}{ Erica arborea } & \multicolumn{3}{c}{ Cistus ladanifer } \\
& 1 & TS (\%) & RS (\%) & NRS (\%) & TS (\%) & RS (\%) & NRS (\%) \\
\hline & 5 & 3.94 & 0.17 & 2.76 & 3.33 & 0.12 & 3.21 \\
ChCl:urea & 10 & 4.04 & 0.20 & 3.34 & 3.97 & 0.14 & 3.84 \\
DES MW- & 20 & 6.45 & 0.28 & 6.17 & 6.36 & 0.23 & 6.13 \\
assisted & 30 & 8.15 & 0.31 & 7.84 & 7.03 & 0.28 & 6.75 \\
extraction & 40 & 8.44 & 0.35 & 8.09 & 8.06 & 0.29 & 7.77 \\
& 50 & 8.83 & 0.40 & 8.43 & 8.09 & 0.30 & 7.79 \\
& 60 & 9.19 & 0.41 & 8.78 & 8.45 & 0.33 & 8.13 \\
\hline & 1 & 0.45 & 0.17 & 0.29 & 0.39 & 0.11 & 0.29 \\
Polar aprotic & 5 & 0.61 & 0.18 & 0.43 & 0.40 & 0.11 & 0.28 \\
solvent MW- & 10 & 0.75 & 0.22 & 0.53 & 0.57 & 0.12 & 0.45 \\
assisted & 20 & 2.46 & 0.25 & 2.21 & 2.44 & 0.19 & 2.25 \\
extraction & 30 & 3.29 & 0.28 & 3.01 & 2.44 & 0.19 & 2.25 \\
& 40 & 3.33 & 0.28 & 3.05 & 2.60 & 0.20 & 2.40 \\
& 50 & 3.68 & 0.30 & 3.37 & 2.70 & 0.23 & 2.47 \\
& 60 & 3.74 & 0.34 & 3.40 & 3.22 & 0.23 & 2.99 \\
\hline & 1 & 0.28 & 0.16 & 0.11 & 0.12 & 0.12 & 0.00 \\
DM-assisted & 5 & 0.71 & 0.19 & 0.52 & 0.26 & 0.13 & 0.13 \\
extraction & 10 & 1.05 & 0.28 & 0.77 & 0.63 & 0.16 & 0.47 \\
& 20 & 2.63 & 0.30 & 2.33 & 2.21 & 0.26 & 1.94 \\
& 30 & 3.27 & 0.36 & 2.91 & 2.39 & 0.28 & 2.11 \\
& 50 & 3.32 & 0.37 & 2.95 & 2.64 & 0.29 & 2.35 \\
& 60 & 3.48 & 0.42 & 3.06 & 2.82 & 0.33 & 2.49 \\
& & 3.80 & 0.44 & 3.36 & 2.90 & 0.36 & 2.54 \\
\hline
\end{tabular}

Table 4. Comparison of the sugar concentrations in the shrubs hydrolysates studied herein with values reported by other authors.

\begin{tabular}{|c|c|c|c|c|c|c|}
\hline \multirow{2}{*}{ Component } & \multirow{2}{*}{ Solvent } & \multicolumn{2}{|c|}{ Shrubs } & \multirow{2}{*}{ Corncob } & \multirow{2}{*}{ Bamboo } & \multirow{2}{*}{ References } \\
\hline & & E. arborea & C. Iadanifer & & & \\
\hline \multirow{4}{*}{ Total sugars (\%) } & $\mathrm{ChCl} /$ urea & $2.94-9.19$ & $3.33-8.45$ & $18.6-20.9$ & & Procentese et al. [18] \\
\hline & $\mathrm{DMAc} / \mathrm{NaHCO}_{3}$ & $0.45-3.74$ & $0.39-2.70$ & & & \\
\hline & $\mathrm{DMAC} / \mathrm{CH}_{3} \mathrm{OK}$ & $0.28-3.80$ & $0.12-2.90$ & & & \\
\hline & $\mathrm{NaOH}$ & 4.63 & 5.64 & & & \\
\hline \multirow{4}{*}{ Reducing sugars (\%) } & $\mathrm{ChCl} /$ urea & $0.17-0.41$ & $0.12-0.33$ & & & \\
\hline & $\mathrm{DMAc} / \mathrm{NaHCO}_{3}$ & $0.17-0.34$ & $0.11-0.23$ & & 3.4 & Wu et al. [19] \\
\hline & $\mathrm{DMAc} / \mathrm{CH}_{3} \mathrm{OK}$ & $0.16-0.44$ & $0.12-0.36$ & & & \\
\hline & $\mathrm{NaOH}$ & 1.29 & 1.00 & & & \\
\hline \multirow{4}{*}{ Non-reducing sugars (\%) } & $\mathrm{ChCl} /$ urea & $2.76-8.75$ & $3.21-8.13$ & & & \\
\hline & $\mathrm{DMAc} / \mathrm{NaHCO}_{3}$ & $0.29-3.40$ & $0.28-2.99$ & & & \\
\hline & $\mathrm{DMAc} / \mathrm{CH}_{3} \mathrm{OK}$ & $0.11-3.36$ & $0.00-2.54$ & & & \\
\hline & $\mathrm{NaOH}$ & 3.34 & 4.64 & & & \\
\hline
\end{tabular}

\section{Analysis of kinetic data}

The kinetic coefficients $(k)$, calculated for the different treatments, are reported in Table 5. It may be observed that, in general terms, the highest constants agree with the highest values of production. That is, for the $\mathrm{ChCl} /$ urea treatment, in addition to the highest concentrations of lignin, furfural and reducing sugars, the highest kinetic constants were also obtained -both for E. arborea and C. ladanifer-: $k_{\text {lignin }}$ values of 0.296 and 0.175 , respectively; $k_{\text {furfural }}$ values of 0.319 and 0.065 , respectively; and 
$k_{\text {reducing sugars }}$ of 0.300 and 0.547 , respectively. Another is the case of 5-HMF and reducing sugars, for which the highest formation kinetics were obtained with the DMAc/ $\mathrm{CH}_{3} \mathrm{OK}$ solvent ( $k_{H M F}$ of 4.822 for E. arborea and 0.779 for C. ladanifer; $k_{T S}$ of 1.404 and 1.778, respectively). The difference in the kinetic behavior between furfural and 5-HMF has to be referred to the different percentages of pentose in the raw materials [20].

Table 5. Kinetic coefficients (k), correlation coefficients (r2) and initial concentration of each sample $\left(H_{o}\right)$ determined from the concentration as a function of time for lignin, furfural, 5-HMF, total sugars and reducing sugars production from the hydrolysis of $E$. arborea and $C$. ladanifer lignocellulosic biomass.

\begin{tabular}{|c|c|c|c|c|c|c|c|c|}
\hline \multirow{2}{*}{ Component } & \multirow{2}{*}{ Solvent } & \multicolumn{3}{|c|}{ E. arborea } & \multicolumn{3}{|c|}{ C. ladanifer } & \multirow{2}{*}{ References } \\
\hline & & $k$ & $r^{2}$ & $H_{0}$ & $k$ & $r^{2}$ & $H_{0}$ & \\
\hline \multirow{3}{*}{ Lignin } & $\mathrm{ChCl} /$ urea & 0.2959 & 0.9707 & 0.0320 & 0.1752 & 0.9479 & 0.0321 & \\
\hline & $\mathrm{DMAc} / \mathrm{NaHCO}_{3}$ & 0.2118 & 0.8583 & 0.0321 & 0.0088 & 0.8646 & 0.0321 & \\
\hline & $\mathrm{DMAc} / \mathrm{CH}_{3} \mathrm{OK}$ & 0.0348 & 0.9745 & 0.0321 & 0.0042 & 0.9831 & 0.0320 & \\
\hline \multirow{3}{*}{ Furfural } & $\mathrm{ChCl} /$ urea & 0.3192 & 0.8214 & 0.0320 & 0.0649 & 0.8900 & 0.0321 & 0.2712 (macauba pulp) [16] \\
\hline & $\mathrm{DMAc} / \mathrm{NaHCO}_{3}$ & 0.0011 & 0.8500 & 0.0321 & 0.0001 & 0.9905 & 0.0321 & \\
\hline & $\mathrm{DMAc} / \mathrm{CH}_{3} \mathrm{OK}$ & 0.0433 & 0.8908 & 0.0321 & 0.0309 & 0.8782 & 0.0320 & \\
\hline \multirow{3}{*}{ 5-HMF } & $\mathrm{ChCl} /$ urea & 0.3844 & 0.9025 & 0.0320 & 0.3296 & 0.8365 & 0.0321 & $\begin{array}{c}0.2729 \text { (macauba pulp), } 0.0810 \\
\text { (macauba shell) [16] }\end{array}$ \\
\hline & $\mathrm{DMAc} / \mathrm{NaHCO}_{3}$ & 0.0025 & 0.6806 & 0.0321 & 0.0013 & 0.9240 & 0.0321 & \\
\hline & $\mathrm{DMAc} / \mathrm{CH}_{3} \mathrm{OK}$ & 4.8222 & 0.8024 & 0.0321 & 0.7798 & 0.8367 & 0.0320 & \\
\hline \multirow{3}{*}{ Total sugars } & $\mathrm{ChCl} /$ urea & 0.3778 & 0.8704 & 0.0100 & 0.1605 & 0.9149 & 0.0100 & \\
\hline & $\mathrm{DMAc} / \mathrm{NaHCO}_{3}$ & 1.4143 & 0.8309 & 0.0100 & 1.3890 & 0.8024 & 0.0100 & \\
\hline & $\mathrm{DMAc} / \mathrm{CH}_{3} \mathrm{OK}$ & 1.4044 & 0.8928 & 0.0100 & 1.7780 & 0.8634 & 0.0100 & \\
\hline \multirow{3}{*}{$\begin{array}{l}\text { Reducing } \\
\text { sugars }\end{array}$} & $\mathrm{ChCl} /$ urea & 0.3005 & 0.8780 & 0.0667 & 0.5469 & 0.9137 & 0.0668 & \\
\hline & $\mathrm{DMAc} / \mathrm{NaHCO}_{3}$ & 0.1600 & 0.8976 & 0.0668 & 0.6234 & 0.8339 & 0.0668 & \\
\hline & $\mathrm{DMAc} / \mathrm{CH}_{3} \mathrm{OK}$ & 0.4351 & 0.9132 & 0.0668 & 0.9528 & 0.8690 & 0.0668 & \\
\hline
\end{tabular}

\section{Mechanisms}

Although the three solvents under study have the ability to disrupt the hydrogen bond network of biopolymers, their different mechanisms result in different efficiencies. Further, the lower performance of DMAc systems can be explained by fact that they are disturbed by water impurities [21].

In the DES system, $\mathrm{ChCl}$ may act as a bridge between the urea and the biomass biopolymers units to, subsequently, weaken and break the specific linkages into the biopolymer (e.g., the ether linkages between the phenylpropane units present in lignin, as reported Alvarez-Vasco et al. [22]). Another possibility would be that, instead of $\mathrm{ChCl}$ and urea, the intermediate agents were choline cation and $\left[\mathrm{Cl}(\text { urea })_{2}\right]^{-}$anion.

Other is the case of DMAc systems, where the hydroxyl groups of lignocellulosic materials may interact with a sodium- or potassium-DMAc macrocation via hydrogen bonding bridged by the bicarbonate or methoxide anions (Figure 5). Sodium or potassium can interact with the carbonyl oxygen via ion-dipole interaction [23], but for this interaction to take place, no biopolymer bound water can be present. On the contrary, in the case of DES systems, this problem does not occur: since water is linked to urea through hydrogen bonding, the deleterious water effect is suppressed [24]. 

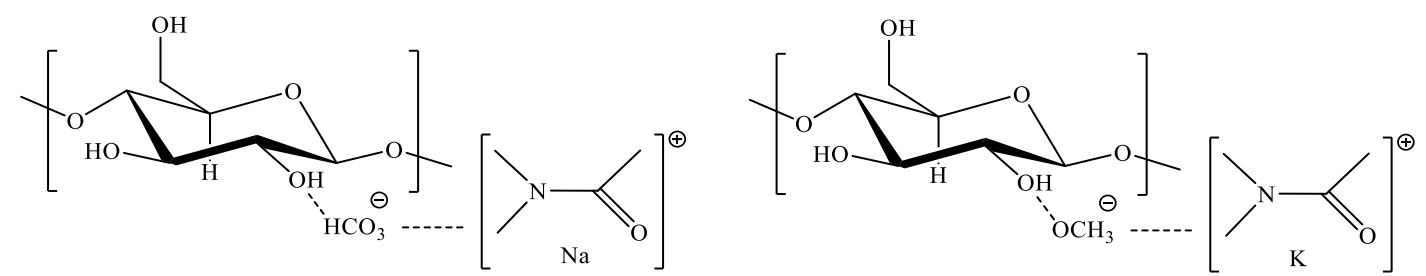

Figure 5. Proposed interaction between $\mathrm{DMAc}-\mathrm{NaHCO}_{3}$ and $\mathrm{DMAc}-\mathrm{CH}_{3} \mathrm{OK}$ solvents and sugar polymer

\section{Conclusions}

The results suggest that the DES treatment offers an efficient, safe, sustainable, and cost effective alternative to conventional methods for the extraction of bioactive compounds from $C$. ladanifer and E. arborea biomass. Samples of these shrubs may be easily dissolved by a $\mathrm{MW}$-assisted procedure in a $\mathrm{ChCl} /$ urea deep eutectic solvent to give lignin, furfural, 5-(hydroxymethyl)furfural and sugars with reasonable yields. Conversely, the DMAc/ $\mathrm{NaHCO}_{3}$ and $\mathrm{DMAc} / \mathrm{CH}_{3} \mathrm{OK}$ solvent exchange systems would not be appropriate due to water impurities disruptive effect.

A peculiarity of the present work compared to previous ones $[15,16]$ is that the current operating conditions led to higher contents of non-reducing sugars than reducing sugars. This finding can be useful to modify cured phenol-formaldehyde resins: whereas reduced sugars cannot be used to modify these resins, non-reducing sugars can be used to replace a major portion of the adhesive resin. Another application of the non-reducing sugars (sucrose) is their advantageous application as a starting material in bioprocesses to produce succinic acid (one of the chemical platforms suggested by the DOE), farnesene (sesquiterpenes) and sucralose.

\section{References}

[1] C.A. Mooney, S.D. Mansfield, R.P. Beatson, J.N. Saddler, The effect of fiber characteristics on hydrolysis and cellulase accessibility to softwood substrates, Enzyme Microb. Technol., 25 (1999) 644-650.

[2] Y. Sun, J. Cheng, Hydrolysis of lignocellulosic materials for ethanol production : a review q, Bioresour. Technol., 83 (2002) 1-11.

[3] Z. Hu, Z. Wen, Enhancing enzymatic digestibility of switchgrass by microwaveassisted alkali pretreatment, Biochem. Eng. J., 38 (2008) 369-378.

[4] D. Jackowiak, J.C. Frigon, T. Ribeiro, A. Pauss, S. Guiot, Enhancing solubilisation and methane production kinetic of switchgrass by microwave pretreatment, Bioresour. Technol., 102 (2011) 3535-3540.

[5] S.M. Nomanbhay, R. Hussain, K. Palanisamy, Microwave-Assisted Alkaline Pretreatment and Microwave Assisted Enzymatic Saccharification of Oil Palm Empty Fruit Bunch Fiber for Enhanced Fermentable Sugar Yield, Journal of Sustainable Bioenergy Systems, 03 (2013) 7-17.

[6] A.d.1. Hoz, A. Diaz-Ortiz, A. Moreno, Microwaves in organic synthesis. Thermal and non-thermal microwave effects, Chem. Soc. Rev., 34 (2005) 164-178.

[7] D.R. Keshwani, J.J. Cheng, Microwave-based alkali pretreatment of switchgrass and coastal bermudagrass for bioethanol production, Biotechnol. Progr., 26 (2010) 644-652. 
[8] A.K. Chandel, C. Es, R. Rudravaram, M.L. Narasu, V. Rao, P. Ravindra, Economics and environmental impact of bioethanol production technologies: an appraisal, Biotechnology and Molecular Biology Review, 2 (2007) 14-32.

[9] H. Lee, D.H. Cho, Y.H. Kim, S.J. Shin, S.B. Kim, S.O. Han, J. Lee, S.W. Kim, C. Park, Tolerance of Saccharomyces cerevisiae K35 to lignocellulose-derived inhibitory compounds, Biotechnology and Bioprocess Engineering, 16 (2011) 755760 .

[10]A. Martinez, M.E. Rodriguez, S.W. York, J.F. Preston, L.O. Ingram, Use of UV absorbance to monitor furans in dilute acid hydrolysates of biomass, Biotechnol. Progr., 16 (2000) 637-641.

[11] S. Sun, Y. Song, P. Sun, J. Chen, Furfural, acid-soluble lignin and reducing sugar content in biomass hydrolysate by spectrophotometric method, Taiyangneng Xuebao/Acta Energiae Solaris Sinica, 34 (2013) 331-336.

[12]C. Chi, Z. Zhang, H.-m. Chang, H. Jameel, Determination of furfural and hydroxymethylfurfural formed from biomass under acidic conditions, Journal of Wood Chemistry and Technology, 29 (2009) 265-276.

[13]G.L. Miller, Use of dinitrosalicylic acid reagent for determination of reducing sugar, Anal. Chem., 31 (1959) 426-428.

[14]M. DuBois, K.a. Gilles, J.K. Hamilton, P.a. Rebers, F. Smith, Colorimetric method for determination of sugars and related substances, Anal. Chem., 28 (1956) 350356.

[15] V. da Silva Lacerda, J.B. López-Sotelo, A. Correa-Guimarães, P. Martín-Ramos, S. Hernández-Navarro, M. Sánchez-Bascones, L.M. Navas-Gracia, Efficient microwave-assisted acid hydrolysis of lignocellulosic materials into total reducing sugars in ionic liquids, Cellulose, Chemistry and Technology, 50 (2016) 761-770.

[16]V. da Silva Lacerda, J.B. López-Sotelo, A. Correa-Guimarães, S. HernándezNavarro, M. Sánchez-Bascones, L.M. Navas-Gracia, P. Martín-Ramos, E. PérezLebeña, J. Martín-Gil, A kinetic study on microwave-assisted conversion of cellulose and lignocellulosic waste into hydroxymethylfurfural/furfural, Bioresource Technology, 180 (2015) 88-96.

[17]A. Martel, Transformation of Symphytum officinale L. and Panicum virgatum L. biomass to 5-Hydroxymethylfurfural for biofuel production, in, 2016.

[18]A. Procentese, E. Johnson, V. Orr, A. Garruto Campanile, J.A. Wood, A. Marzocchella, L. Rehmann, Deep eutectic solvent pretreatment and subsequent saccharification of corncob, Bioresour. Technol., 192 (2015) 31-36.

[19] Y. Wu, C. Zhang, Y. Liu, Z. Fu, B. Dai, D. Yin, Biomass char sulfonic acids (BC$\mathrm{SO} 3 \mathrm{H}$ )-catalyzed hydrolysis of bamboo under microwave irradiation, BioResources, 7 (2012) 5950-5959.

[20]Y. Liu, J. Wang, M. Wolcott, Modeling the production of sugar and byproducts from acid bisulfite pretreatment and enzymatic hydrolysis of Douglas-fir, Bioresour. Technol., 224 (2017) 389-396.

[21]A. Potthast, T. Rosenau, R. Buchner, T. Röder, G. Ebner, H. Bruglachner, H. Sixta, P. Kosma, The cellulose solvent system N,N-dimethylacetamide/lithium chloride revisited: The effect of water on physicochemical properties and chemical stability, Cellulose, 9 (2002) 41-53.

[22] C. Alvarez-Vasco, R. Ma, M. Quintero, M. Guo, S. Geleynse, K.K. Ramasamy, M. Wolcott, X. Zhang, Unique low-molecular-weight lignin with high purity extracted from wood by deep eutectic solvents (DES): a source of lignin for valorization, Green Chem., 18 (2016) 5133-5141. 
[23]C.L. McCormick, P.A. Callais, B.H. Hutchinson, Solution studies of cellulose in lithium chloride and N,N-dimethylacetamide, Macromolecules, 18 (1985) 23942401.

[24]C. Du, B. Zhao, X.-B. Chen, N. Birbilis, H. Yang, Effect of water presence on choline chloride-2urea ionic liquid and coating platings from the hydrated ionic liquid, Sci. Rep., 6 (2016) 29225-29225. 

6.CONCLUSIONES 

De los resultados del presente estudio se han alcanzado las siguientes conclusiones:

1. Del muestreo de los arbustedos de Ayoó de Vidriales (Zamora, España), se han establecido las características poblacionales, densidad y estructuras de edad para jara (Cistus ladanifer) y brezo (Erica arborea). A partir del análisis ortofotográfico de la superficie, cabe destacar que la densidad poblacional de ambas especies es tan elevada que la fracción de cavidad cubierta es total.

2. En relación con la caracterización química a través de análisis elemental (CHN) de hojas, tallos delgados, tallos gruesos y raíces de ambas especies, se ha confirmado que los contenidos de carbono en las fracciones aérea y radical no difieren de forma significativa, por lo que el uso del valor global de 0.5 recomendado por la IPCC es aplicable. Con respecto a los contenidos de nitrógeno, se observa una distribución de pirámide invertida, con los porcentajes más altos en las hojas y los más bajos en las raíces. Ahora bien, al analizar el contenido en nitrógeno teniendo en cuenta la biomasa de cada fracción, se ha observado que el comportamiento es muy diferente en ambas especies: para $C$. ladanifer sigue el orden hojas $>$ tallos delgados $>$ tallos gruesos $>$ raíces, mientras para E. arborea es tallos gruesos $>$ raíces $>$ tallos delgados $>$ hojas.

3. Se han determinado los cocientes root-to-shoot para la estimación de la biomasa total a partir de medidas de biomasa aérea mediante métodos directos e indirectos. Los cocientes obtenidos por el método UN-ECE/FAO-IPCC/Sanquetta et al. a partir de las determinaciones de biomasa han resultado ser de 0.22 para $C$. ladanifer y 0.55 para E. arborea, en consonancia con las notables diferencias en cuanto a porcentajes de distribución de biomasa para ambas especies. Por otra parte, el análisis vibracional de las muestras mediante espectroscopía infrarroja con transformada de Fourier (FTIR), una técnica no destructiva, rápida y de bajo coste, ha permitido obtener excelentes estimaciones de los cocientes root-to-shoot. Mediante el uso de las áreas bajo los picos de absorción de $1369 \mathrm{~cm}^{-1}$ (banda asociada a la celulosa) y de $1514 \mathrm{~cm}^{-1}$ (banda asociada a lignina), para $C$. ladanifer y E. arborea, respectivamente, se han obtenido cocientes de 0.25 y 0.53 , resultados prácticamente idénticos a los obtenidos por el método tradicional.

4. En base a la información de contenidos de carbono y distribución de la biomasa, ha sido posible estudiar el comportamiento como sumideros de carbono de estos 
arbustedos. Se ha estimado que C. ladanifer and E. arborea almacenarían 12.27 y $19.86 \mathrm{tC} \cdot \mathrm{ha}^{-1}$, o 45 y $73 \mathrm{tCO}_{2} \mathrm{eq} \cdot \mathrm{ha}^{-1}$, valores sustancialmente más altos que los de otros arbustos, evidenciando la importancia de estas dos especies para la mitigación del Cambio Climático.

5. El aprovechamiento como combustible de E. arborea cumple los requerimientos EN 14961-2, pues los contenidos caloríficos de núcleo y corteza son muy altos y no significativamente diferentes y el contenido en cenizas para ejemplares con diámetro $>2.8 \mathrm{~cm}$ es admisible. Otro es el caso de C. ladanifer, que se encuentra en el límite permitido de la normativa y sólo cumple los requerimientos para ejemplares añosos, cuando el diámetro de la madera es bastante grande $(>3.4 \mathrm{~cm})$ y el porcentaje de corteza es inferior al 16\%. Sólo en esas condiciones los porcentajes de cenizas resultan aceptables $(\leq 2 \%)$ y el contenido calorífico alcanza un valor admisible para su uso como combustible $(18 \mathrm{~kJ} / \mathrm{g})$. En consecuencia, mientras la cosecha de E. arborea para utilización como fuel no tiene por qué resultar selectiva, la de $C$. ladanifer debería limitarse a los ejemplares más robustos.

6. La investigación de las dos especies estudiadas como precursores de productos de alto valor añadido ha conducido al estudio de sus aceites esenciales, que alcanzan precios de mercado superiores a $200 € / \mathrm{L}$ en el caso de $C$. ladanifer. La caracterización de sus componentes químicos principales, realizada en comparación con otras partes de las plantas y con aceites, gomas y resinas de otras especies, ha sido llevada a cabo por FTIR. La localización específica de las bandas de absorción para grupos oxo $\alpha: \beta$ insaturados ha sido relacionado con el diferente contenido en terpenoides de los aceites: mono- y sesquiterpenoides para C. ladanifer y triterpenoides para E. arborea.

7. A efectos de incendios forestales, la inflamabilidad de los arbustos bajo estudio (y, por extensión, la de sus resinas y aceites) ha de ser referida (aparte de tiempo de ignición y porcentaje de humedad) a su contenido en los diversos tipos de terpenoides y a la concentración con que estas especies tipo isoprenoide son emitidas al aire. La alta inflamabilidad de E. arborea ha de ser referida a sus altas emisiones de terpenos durante todo el año (que pueden alcanzar hasta 40 $\mu \mathrm{g} \cdot \mathrm{gDM}^{-1} \cdot \mathrm{h}^{-1}$ ) por más que su composición sea relativamente rica en terpenoides de baja volatilidad, como los triterpenoides. Por el contrario, C. ladanifer, aunque 
emite al aire cantidades modestas de terpenoides $\left(<7 \mu \mathrm{g} \mathrm{gDM}^{-1} \mathrm{~h}^{-1}\right)$, posee una inflamabilidad muy alta en verano debido a la emisión potenciada de $\alpha$-pineno, un monoterpeno especialmente volátil, componente importante de su aceite esencial.

8. El análisis térmico ha evidenciado una mayor estabilidad del aceite de $C$. ladanifer frente al de E. arborea. Pese a la termolabilidad de los terpenoides, la hidrodestilación ha resultado ser un procedimiento adecuado para su extracción, al no afectar a su persistencia.

9. De los sistemas de hidrólisis y extracción utilizados para la conversión de la biomasa de C. ladanifer y E. arborea en productos derivados, el sistema cloruro de colina/urea- $\mathrm{HNO}_{3}, 10 \%$ asistido por microondas ha proporcionado mejores resultados en lignina, furfural, hidroximetilfurfural y azúcares que los sistemas DMAc/ $\mathrm{NaHCO}_{3}$ y DMAc/CH 3 OK, también asistidos por microondas.

Sin embargo, si el objetivo de tratar la biomasa de C. ladanifer y E. arborea es recuperar azúcares para subsiguiente sacarificación enzimática, los muy bajos contenidos en 5-HFM obtenidos con los sistemas dimetilacetamida (especialmente, $\mathrm{CH}_{3} \mathrm{OK}$ ) hacen de estos unos procedimientos ventajosos en comparación con el método tradicional que utiliza $\mathrm{NaOH}$.

10. Los índices de cristalinidad del residuo celulósico que resulta tras tratamiento por cloruro de colina/urea asistido por microondas son mejores que los que resultan del tratamiento alcalino. La información obtenida de los espectros ATR-FTIR, imágenes SEM y datos de accesibilidad permite establecer que las cristalinidades de las fibras de celulosa procedentes de E. arborea y C. ladanifer se encuentran dentro del intervalo establecido para pulpa de madera y por consiguiente, pueden ser de aplicación en la industria del empaquetado y como reforzantes en materiales compuestos. 

From the results of the present study the following conclusions have been reached:

1. Population characteristics, density and age structures for gum rockrose (Cistus ladanifer) and heather (Erica arborea) have been established from the sampling of shurblands in Ayoó de Vidriales (Zamora, Spain). From the orthophotographic analysis of the surface, it is worth noting that the population density of both species is so high that the entire soil is covered by the plant canopies.

2. In relation to the chemical characterization of leaves, thin stems, thick stems and roots of both species by elemental analysis $(\mathrm{CHN})$, it has been confirmed that the carbon contents in the aerial and radical fractions do not differ significantly, so the use of the global value of 0.5 recommended by the IPCC is applicable. With respect to the nitrogen contents, an inverted pyramid distribution is observed, with the highest percentages in the leaves and the lowest in the roots. However, when analyzing the nitrogen contents taking into account the biomass of each fraction, it has been observed that the behavior is very different in both species: for $C$. ladanifer follows the order leaves $>$ thin stems $>$ thick stems $>$ roots, while for $E$. arborea is thick stems $>$ roots $>$ thin stems $>$ leaves.

3. Root-to-shoot ratios have been determined for the estimation of total biomass from aerial biomass measurements using direct and indirect methods. The ratios obtained by the UN-ECE/FAO-IPCC/Sanquetta et al. from biomass determinations have been found to be 0.22 for $C$. ladanifer and 0.55 for $E$. arborea, in consonance with the notable differences in percentages of biomass distribution for both species. On the other hand, the vibrational analysis of samples using Fourier Transform Infrared Spectroscopy (FTIR), a nondestructive, fast and low-cost technique, has allowed to obtain excellent estimates of the root-to-shoot ratios. By using the areas under the absorption peaks of 1369 $\mathrm{cm}^{-1}$ (band associated with cellulose) and $1514 \mathrm{~cm}^{-1}$ (lignin-associated band), for C. ladanifer and E. arborea, respectively, ratios of 0.25 and 0.53 were obtained, i.e., almost identical results to those obtained by the traditional method.

4. Based on information on carbon content and biomass distribution, it has been possible to study the behavior as carbon sinks of these shrubs. It has been estimated that $C$. ladanifer and E. arborea would store 12.27 and $19.86 \mathrm{tC} \cdot \mathrm{ha}^{-1}$, or 
45 and $73 \mathrm{tCO}_{2} \mathrm{eq} \cdot \mathrm{ha}^{-1}$, values substantially higher than those of other shrubs, evidencing the importance of these two species for the mitigation of Climate Change.

5. The use of E. arborea as fuel fulfills the requirements of EN 14961-2, since the calorific contents of core and bark are very high and not significantly different and the ash content for specimens with a diameter $>2.8 \mathrm{~cm}$ is admissible. Another is the case of $C$. ladanifer, which is within the limits allowed by the regulations and only meets the requirements for elderly specimens, when the diameter of the wood is quite large $(>3.4 \mathrm{~cm})$ and the percentage of bark is less than $16 \%$. Only under these conditions the ash percentages are acceptable $(\leq 2 \%)$ and the calorific content reaches an admissible value for use as fuel $(18 \mathrm{~kJ} / \mathrm{g})$. Consequently, while the crop of $E$. arborea for use as fuel does not have to be selective, $C$. ladanifer should be limited to the most robust specimens.

6. The valorization of the two species studied on the basis of high value added products has led to the study of their essential oils, which reach market prices in excess of $200 € / \mathrm{L}$ in the case of $C$. ladanifer. The characterization of their main chemical components, performed in comparison with other parts of plants and with oils, gums and resins of other species, has been carried out by FTIR. The specific location of the absorption bands for oxo $\alpha$ : $\beta$ unsaturated groups has been related to the different terpenoid content of oils: mono- and sesquiterpenoids for C. ladanifer and triterpenoids for E. arborea.

7. As regards forest fires, the flammability of the shrubs under study (and, by extension, that of their resins and oils) must be referred to (in addition to ignition time and percentage of moisture) their content in terpenoids and the concentration with which these isoprenoid type species are emitted into the air. The high flammability of E. arborea has to be referred to its high terpene emissions throughout the year (which can reach up to $40 \mu \mathrm{g} \cdot \mathrm{gDM}^{-1} \cdot \mathrm{h}^{-1}$ ), even though its composition is relatively rich in low volatility terpenoids, such as triterpenoids. In contrast, C. ladanifer, although emitting modest quantities of terpenoids $(<7$ $\left.\mu \mathrm{g} \cdot \mathrm{gDM}^{-1} \cdot \mathrm{h}^{-1}\right)$, has a very high flammability in summer due to the enhanced emission of $\alpha$-pinene, a particularly volatile monoterpene component important part of its essential oil. 
8. The thermal analysis showed a greater stability of the oil of $C$. ladanifer as compared to that of E. arborea. Despite the thermolability of terpenoids, hydrodistillation has proven to be a suitable procedure for its extraction, as it does not affect its persistence.

9. From the hydrolysis and extraction systems used for the conversion of $C$. ladanifer and E. arborea biomass to by-products, the microwave-assisted choline/urea- $\mathrm{HNO}_{3}$ chloride system has attained better results in terms of lignin, furfural, hydroxymethylfurfural and sugars production than the microwave assisted $\mathrm{DMAc} / \mathrm{NaHCO}_{3}$ and $\mathrm{DMAc} / \mathrm{CH}_{3} \mathrm{OK}$ systems.

Nevertheless, if the aim of treating $C$. ladanifer and E. arborea biomass is to recover sugars for subsequent enzymatic saccharification, the very low 5-HMF contents attained with the dimetylacetamide systems (especially $\mathrm{CH}_{3} \mathrm{OK}$ ) make them highly advantageous as compared to the traditional method using $\mathrm{NaOH}$.

10. The crystallinity indices of the cellulosic residue resulting from treatment by microwave assisted choline chloride/urea DES are better than those resulting from the alkaline treatment. The information obtained from ATR-FTIR spectra, SEM images and accessibility data makes it possible to establish that the crystallinity of the cellulose fibers from E. arborea and $C$. ladanifer are within the range established for wood pulp and can therefore be of application in the packaging industry and as reinforcers in composite materials. 

7.BIBLIOGRAFÍA 

AFANASEV, N.; PROKSHIN, G.; LICHUTINA, T.; GUSAKOVA, M.; VISHNYAKOVA, A.; SUKHOV, D.; DERKACHEVA, O.Y. 2007. Effect of residual lignin on the supramolecular structure of sulfate hardwood cellulose: a Fourier IR study. Russ. J. Appl. Chem., 80: 1724-1727.

AGREN, G.I.; INGESTAD, T. 1987. Root: shoot ratio as a balance between nitrogen productivity and photosynthesis. Plant, Cell and Environment, 10(7): 579-586. doi: 10.1111/1365-3040.ep11604105.

AHTEE, M.; HATTULA, T.; MANGS, J.; PAAKKARI, T. 1983. An X-ray diffraction method for determination of crystallinity in wood pulp. Paperi Ja Puu, 65: 475-480.

AKHLAGUI, S.P.; BERRY, R.C.; TAM, K.C. 2013. Surface modification of cellulose nanocrystal with chitosan oligosaccharide for drug delivery applications, Cellulose, 20: 1747-1764.

ALENCAR, J.W.; ALVES, P.B.; CRAVEIRO, A.A. 1983. Pyrolysis of tropical vegetable oils. Journal of Agricultural and Food Chemistry, 31: 1268-1270.

ALÍAS GALLEGO, J.C.; GARCÍA ROSA, M., VALARES MASA, C.; SOSA DÍAZ, T.; CHAVES LOBÓN, N. El matorral como sumidero de carbono. $5^{\circ}$ Congreso Forestal Español. Ávila, 21-25 Sept, 2009.

AMIR, S.; HADIFI, M.; LEMEE, M.; MERLINA, G.; GUIRESSE, M-; PINELLI, E.; REVEL, J.C.; BAILLY, J.R.; AMBLES, A. 2006. Structural characterization of humic acids, extracted from sewage sludge during composting, by thermochemolysis-gas chromatography-mass spectrometry. Process Biochemistry, 41 (2): 410-422. doi: 10.1016/j.procbio.2005.07.005.

ARSUAGA, J.L.; MARTÍN-LOECHES, M. 2013. El sello indeleble: Pasado, presente y futuro del ser humano. Penguin Random House, 424 pp.

ASEEVA, R.M.; THANH, B.D.; SERKOV, B.B. 2005. Factors Affecting Heat Release at the Combustion of the Different Species of Wood. En: Berlin AA, Novakov IA, Khalturinskiy NA, Zaikov GE (eds.) Chemical Physics of Pyrolysis, Combustion, and Oxidation. Nova Science Publishers New York, USA, pp 45-53.

BAKER, M.J., Júlio Trevisan, Paul Bassan, Rohit Bhargava, Holly J. Butler, Konrad M. Dorling, Peter R. Fielden, Simon W. Fogarty, Nigel J. Fullwood, Kelly a Heys, Caryn Hughes, Peter Lasch, Pierre L. Martin-Hirsch, Blessing Obinaju, Ganesh D. Sockalingum, Josep Sulé-Suso, Rebecca J. Strong, Michael J. Walsh, Bayden R. Wood, Peter Gardner, and Francis L. Martin. 2014. Using Fourier transform IR spectroscopy to analyze biological materials. Nature protocols 9 (8):1771-91. doi: 10.1038/nprot.2014.110.

BALDOCCHI, D.D.; FALGE, E.; GU, L.; OLSON, R.; HOLLINGER, E.; RUNNING, D; ANTHONI, P.M. et al 2001. FLUXNET: A new tool to study the temporal and spatial variability of ecosystem-scale carbon dioxide, water vapor, and energy flux densities. Bulletin of the American Meteorological Society, 82: 2415-2434.

BARBOUTIS, I; LYKIDIS, C. 2014. The effects of bark on fuel characteristics of some evergreen Mediterranean hardwood species. In: Barnes HM, Herian VL (eds) Proceedings of the 57th International Convention of Society of Wood Science and Technology, Zvolen, Slovakia. Society of Wood Science and Technology, pp 533540. 
BARBONI, T.; PELLIZZARO, G.; ARCA, B: CHIARAMONTI, N.; DUCE, P. 2010. Analysis and origins of volatile organic compounds smoke from ligno-cellulosic fuels. Journal of Analytical and Applied Pyrolysis, 89(1): 60-65. doi: 10.1016/j.jaap.2010.05.006.

BARMPOUTIS, P.; LYKIDIS, C.; BARBOUTIS, I. 2015. Influence of stem diameter and bark ratio of evergreen hardwoods on the fuel characteristics of the produced pellets. Pro Ligno 11:673-679.

BARRIO, DUEÑAS et al., Industrial Crops and Products, 2013, 41: 41-45; doi: http://dx.doi.org/10.1016/j.indcrop.2012.03.038

BASANTA, A. 1982. Vegetación serial en Sierra Morena. Estudio ecológico de las respuestas del matorral a distintas intervenciones en el Coto Nacional "La Pata del Caballo" (Huelva). PhD, Universidad de Sevilla.

BENDAHOU, Y.; HABIBI; Y.; KADDAMI, H.; DUFRESNE, A. 2009. PhysicoChemical Characterization of Palm from Phoenix Dactylifera-L, Preparation of Cellulose Whiskers and Natural Rubber-Based Nanocomposites. Journal of Biobased Materials and Bioenergy, 3: 81-90.

BOMBELLI, A. et al. 2009. T12 Assessment of the status of the development of the standards for the Terrestrial Essential Climate Variables: Biomass vol 67, 10 edn. Global Terrestrial Observing System Secretariat, Land and Water Division (NRL), Food and Agriculture Organization of the United Nations (FAO), Rome, Italy.

BOUBAKER, A.; KAYOULI, C.; BOUKARI, A.; BULDGEN, A. 2004. Chemical and biological characterisation of some woody species browsed by goats in the NorthWest of Tunisia. En: Ben Salem H, Morand-Fehr P, Nefzaoui A (eds) Nutrition and feeding strategies of sheep and goats under harsh climates, vol 59. Options Méditerranéennes: Série A. Séminaires Méditerranéens. CIHEAM, Zaragoza, Spain, pp 147-151

BESSAH, R; BENYOUSSEF, E-H. 2014. Essential oil composition of Erica arborea L. leaves from Algeria. Journal of Essential Oil Bearing Plants, 17: 931-935.

BJÖRMAN, A. 1956. Studies on finely divided wood. Part 1. Extraction of lignin with neutral solvents," Svensk papperstidning, 59: 477-485.

BLANCO OYONARTE, P.; NAVARRO CERRILLO, R.M. 2003. Aboveground phytomass models for major species in shrub ecosystems of western Andalusia." Forest Systems, 12 (3):47-55. doi: 10.5424/1078.

BODIRLAU, R.; TEACA, C.A.; SPIRIDON, I. 2009. Preparation and characterization of composites comprising modified hardwood and wood polymers/poly (vinyl chloride). BioResources, 4, 1285-1304.

CHPQA. 2008. Guidance Note 29: Alternative fuels - Energy inputs. UK Combined Heat \& Power Quality Assurance Programme, London, UK.

CANCELLIERI, D.; LEONI, E.; ROSSI, J.L. 2005. Kinetics of the thermal degradation of Erica arborea by DSC: Hybrid kinetic method. Thermochimica Acta, 438: 41-50.

CARRIÓN-PRIETO, P.; MARTIN-RAMOS, P.; TERESA M.R. MARÍA; HERNÁNDEZ-NAVARRO, S.; GARRIDO-LAURNAGA, F.; M. ERMELINDA S. EUSEBIO; MARTÍN-GIL, J. 2016. Vibrational and termal studies of essential oils derived from Cistus ladanifer and Erica arborea. Natural Product Communications, 12(1): 119-122. 
CARRIÓN-PRIETO, P.; HERNÁNDEZ-NAVARRO, S.; MARTIN-RAMOS， P.; SANCHEZ-SASTRE, L.F.; GARRIDO-LAURNAGA, F.; MARCOS-ROBLES, J.L.; MARTÍN-GIL, J. 2017. Mediterranean shrublands as carbon sinks for Climate Change mitigation: new root to shoot ratios. Carbon Management. doi: $10.1080 / 17583004.2017 .1285178$.

CARRIÓN-PRIETO， P.; MARTIN-RAMOS， P.; HERNÁNDEZ-NAVARRO，S.; SÁNCHEZ-SASTRE, L.F.; MARTÍN-GIL, J. 2017. On the crystallinity of cellulose microfibers from alkaline and ionic liquids treatments of $C$. ladanifer and $E$. arborea stems. En prensa.

CARRIÓN-PRIETO, P.; MARTIN-RAMOS, P.; HERNÁNDEZ-NAVARRO, S.; SÁNCHEZ-SASTRE, L.F.; MARCOS-ROBLES, J.L.; MARTÍN-GIL, J. 2017. Valorization of Cistus ladanifer and Erica arborea shrubs for fuel: wood and bark thermal characterization. En prensa.

CASARES, M. 2017. Las cajas de las jaras. Conceptos básicos Universidad de Granada. http://www.ugr.es/ mcasares/Organografia/Fruto/Frutos\%20cerrados.htm

CASTRO-DÍEZ, P-; MONTSERRAT-MARTÍ, G.; CORNELISSEN, J.H.C. 2003. Trade-offs between phenology, relative growth rate, life form and seed mass among 22 Mediterranean woody species. Plant Ecology, 166 (1):117-129. doi: 10.1023/a:1023209230303.

CASTRO, I.; CASADO, M.A.; RAMÍREZ-SANZ, L.; DE MIGUEL, J.M.; COSTA, M.; DÍAZ-PINEDA, F. 1996. Funciones de estimación de la biomasa aérea de varias especies del matorral mediterráneo del centro de la península Ibérica. Orsis: Organismes i Sistemes, 11: 107-116.

CATONI, R.; GRATANI, L. 2014. Variations in leaf respiration and photosynthesis ratio in response to air temperature and water availability among Mediterranean evergreen species. Journal of Arid Environments, 102: 82-88. doi: 10.1016/j.jaridenv.2013.11.013.

CERRILLO, R.M.; NAVARRO, R.M.; BLANCO-OYONARTE, P. 2006. Estimation of above-ground biomass in shrubland ecosystems of southern Spain. Investigación agraria. Sistemas y recursos forestales, 15(2): 197-207. doi: 10.5424/srf/200615200964.

CHANDEL, A.K.; ES, C.; RUDRAVARAM, R.; NARASU, M.L.; RAO, V.; RAVINDRA, P. 2007. Economics and environmental impact of bioethanol production technologies: an appraisal. Biotechnology and Molecular Biology Review, 2: 14-32.

CHEN, H.; FERRARI, C.; ANGIULI, M.; YAO, J.; RASPI, C.; BRAMANTI, E. 2010. Qualitative and quantitative analysis of wood samples by Fourier transform infrared spectroscopy and multivariate analysis." Carbohydrate Polymers, 82(3): 772-778. doi: 10.1016/j.carbpol.2010.05.052.

CHI, C.; ZHANG, Z.; CHANG, H.M.; JAMEEL, H. 2009. Determination of furfural and hydroxymethylfurfural formed from biomass under acidic conditions. Journal of Wood Chemistry and Technology, 29: 265-276.

DE LA HOZ, A.; DÍAZ-ORTIZ, A. MORENO, A. 2005. Microwaves in organic synthesis. Thermal and non-thermal microwave effects. Chemical Society Reviews, vol. 34, pp. 164-178. 
DE LA ROSA, J.M, GONZÁLEZ-PÉREZ，J.A.; GONZÁLEZ-VÁZQUEZ， R.; KNICKER, H.; LÓPEZ.CAPEL, E.; MANNING, D.A.C.; GONZÁLEZ-VILA, F.J. 2008. Use of pyrolysis/GC-MS combined with thermal analysis to monitor $C$ and $N$ changes in soil organic matter from a Mediterranean fire affected forest. Catena, 74: 296-303.

DE MELlO, A.; NUTTO, L.; WEBER, C.; SANQUETTA, C.; MONTEIRO DE MATOS, J.; BECKER, G. 2012. Individual biomass and carbon equations for Mimosa scabrella Benth. (bracatinga) in southern Brazil. Silva Fennica, 46(3): 333-343. doi: 10.14214/sf.44.

DE MESQUITA, J.P; DONNICI, C.L.; PEREIRA, F.V. 2010. Biobased nanocomposites from layer-by-layer assembly of cellulose nanowhiskers with chitosan, Biomacromolecules, 11: 473-480.

DENCE, C.V. 1992. The Determination of Lignin, D. S. Y. Lin and P. E. D. C. W. Dence, Eds., ed: Springer Berlin Heidelberg, pp. 33-61.

DICASTRI, F. 1981. Mediterranean--type shrublands of the World. En: Mediterraneantype Shrublands. F. Di Castri, D.W. Goodall and R.L. Specht, R.L., eds. pp 1-152. Elsevier, Amsterdam.

DIMITRAKOPOULOS, A.P.; PANOV, P.I. 2001. Pyric properties of some dominant Mediterranean vegetation species. Int $\mathrm{J}$ Wildland Fire, 10: 23-27. doi:10.1071/wf01003.

DISCOVER LIFE. http://www.discoverlife.org/mp/20m?kind=Erica+arborea

DOAT, J.; VALETTE, J.C.; ASKRI, D.; CAUMARTIN, L.; BETTACHINI, M.; MORO, M. 1981. Le pouvoir calorifique supérieur d'espèces forestières méditerranéennes. Annales des Sciences forestières, 38:469-486.

DUBOIS, M: GILlES, K.A.; HAMILTON, J.K.; REBERS, P.A.; SMITH, F. 1956. Colorimetric method for determination of sugars and related substances. Analytical Chemistry, 28: 350-356, 1956.

ECN. 2016. ECN Phyllis2 database for biomass and waste. Energy research Centre of the Netherlands. https://www.ecn.nl/phyllis2. Accessed December 3, 20162016

EN-PLUS. 2015. ENplus Handbook version 3.0. European Biomass Association AEBIOM, Brussels, Belgium.

ESPAÑA, 2007. Ley orgánica 14/2007, de 30 de noviembre, de reforma del Estatuto de Autonomía de Castilla y León. Boletín Oficial del Estado, 01/12/2007, núm. 288.

EUROPEAN PELLET COUNCIL. 2011. Handbook for the Certification of Wood Pellets for Heating Purposes, based on EN 14961-2. European Pellet Council, Brussels, Belgium

EVANS, R.; NEWMAN, R.H.; ROICK, U.C.; SUCKLING, I.D.; WALLIS, A.F.A. 1995. Changes in Cellulose Crystallinity During Kraft Pulping. Comparison of Infrared, X-ray Diffraction and Solid State NMR Results. Holzforschung 49: 498504.

EVANS, P.A. 1991. Differentiating "hard" from "soft" woods using Fourier transform infrared and Fourier transform spectroscopy. Spectrochimica Acta Part A: Molecular Spectroscopy 47 (9-10):1441-1447. doi: 10.1016/0584-8539(91)80235-b. 
FAN, M.; DAI, D.; HUANG, B. 2012. Fourier transform infrared spectroscopy for natural fibres. In Fourier transform-materials analysis. Salih S.M. (Ed.), InTech, Rijeka (Croatia), 45-68.

FAUST, B. 1997. Modern Chemical Techniques: An Essential Reference for Students and Teachers: Royal Society of Chemistry.

FEDERICI, S.; TUBIELLO, F.N.; SALVATORE, M.; JACOBS, H.; SCHMIDHUBER, J. 2015. New estimates of $\mathrm{CO}_{2}$ forest emissions and removals: 1990-2015." Forest Ecology and Management 352:89-98. doi: 10.1016/j.foreco.2015.04.022.

FERRO, M.D.; FERNANDES, M.C.; PAULINO, A.F.C.; PROZIL, S.O.; GRAVITIS, J.; EVTUGUIN, D.V.; XAVIER, A.M.R.B. 2015. Bioethanol production from steam explosion pretreated and alkali extracted Cistus ladanifer (rockrose). Biochemical Engineering Journal, 104: 98-105. doi: 10.1016/j.bej.2015.04.009.

FRENCH, A.D.; SANTIAGO CINTRÓN, M. 2012. Cellulose polymorphy, crystallite size, and the Segal Crystallinity Index, Cellulose, 20: 583-588.

GARCÍA-ROSA, M. 2013. Estudio de la biomasa de Cistus ladanifer L. y Retama sphaerocarpa L. como sumidero de CO2: existencias y potencialidad. Departamento de Biología Vegetal, Ecología y Ciencias de la Tierra, Universidad de Extremadura, Universidad de Extremadura. España (http://hdl.handle.net/10662/577).

GAYOSO, A.; GUERRA, G. 2005. Contenido de carbono en la biomasa aérea de bosques nativos en Chile. Bosque (Valdivia), 26(2): 33-38. doi: 10.4067/s071792002005000200005.

GOMES, PB; MATA, VG; RODRIGUES, A.E. 2005. Characterization of the Portuguese-grown Cistus ladanifer essential oil. Journal of Essential Oil Research, 17: 160-165.

GRIFFTHS, P.R.; DE HASETH, J.A. 2007. Fourier Transform Infrared Spectrometry: John Wiley \& Sons.

GÜLZ, P-G; KOBOLD, U; MICHAELIS, K; VOSTROWSKY, O. 1984. The composition of terpene hydrocarbons in the essential oils from leaves of four Cistus species. Zeitschrift für Naturforschung C, 39: 699-704.HEGNAUER, R; HEGNAUER, M. 1962. Chemotaxonomie der Pflanzen; eine Übersicht über die Verbreitung und die systematische Bedeutung der Pflanzenstoffe, Birkhäuser, Basel.

HERNÁNDEZ, S.; MARTÍN, J.; RUIZ, N. 2007. Estimación del secuestro de carbono en suelos bajo masas forestales de Pinus halepensis en Castilla y León (España). Departamento de Ingeniería Agrícola y Forestal. Universidad de Valladolid. Reunión de la sociedad española forestal.

HIDALGO, N. 2014. Brezo blanco. Zeta bolsillo, 336 pp.

HILBERT, D.W. 1990. Optimization of Plant Root: Shoot Ratios and Internal Nitrogen Concentration." Annals of Botany 66(1): 91-99.

HUCK, C.W. 2015. Advances of infrared spectroscopy in natural product research. Phytochemistry Letters, 11: 384-393. doi: 10.1016/j.phytol.2014.10.026.

IBAÑEZ, J.J.; VAYREDA, J.; GRACIA, C. 2002. Metodología complementaria al Inventario Forestal Nacional en Catalunya." In El Inventario Forestal Nacional. Elemento clave para la Gestión Forestal Sostenible, edited by F. Bravo, M. Del Río and C. Del Peso, 67-77. Fundación General de la Universidad de Valladolid. 
IDAE. Resumen del Plan de Energías Renovables 2011-2022.

http://www.idae.es/uploads/documentos/documentos Resumen PER 20112020 15f3dad6.pdf

IPCC. 2006. 2006 IPCC Guidelines for National Greenhouse Gas Inventories Agriculture, Forestry and Other Land Use. Edited by H.S. Eggelston, L. Buendia, K. Miwa, T. Ngara and K. Tanabe. Vol. 4, Guidelines for National Greenhouse Gas Inventories. Hayama, Japan: IPCC National Greenhouse Gas Inventories Programme - Institute for Global Environmental Strategies.

ISO 2014. Solid biofuels, Fuel specifications and classes. Part 2: Graded wood pellets. ISO 17225-2:2014. International Organization for Standardization, Geneva, Switzerland.

ISO 2015. Solid biofuels, Determination of ash content. ISO 18122:2015. International Organization for Standardization, Geneva, Switzerland.

JACKOWIAK, D.; FRIGON, J.C.; RIBEIRO, T.; PAUSS, A.; GUIOT, S. 2011. Enhancing solubilisation and methane production kinetic of switchgrass by microwave pretreatment. Bioresource Technology, 102: 3535-3540

JOVIĆ, O.; SMOLIĆ, T; JURIŜIĆ, Z; MEIĆ, Z.; HRENAR, T. 2013. Chemometric analysis of Croatian extra virgin olive oils from central Dalmatia region. Croatica Chemica Acta, 86: 335-344.

JU, X.; BOWDEN, M.; BROWN, E.E.; ZHANG, X. 2015. An improved X-ray diffraction method for cellulose crystallinity measurement. Carbohydr. Polym., 123: 476-481.

JUNTA DE CASTILLA Y LEÓN. Programa de movilización de los recursos forestales en Castilla y León, 2014-2022. Consejería de Fomento y Medio Ambiente, Valladolid, $68 \mathrm{pp}$.

KAMIDE, K. 2005. Cellulose and cellulose derivatives: molecular characterization and its applications, 1st ed., Elsevier, Amsterdam, 652 pp.

KAUPPI, P.E.; MIELIKAINEN, K.; KUUSELA, K. 1992. Biomass and Carbon Budget of European Forests, 1971 to 1990. Science, 256 (5053):70-74. doi: 10.1126/science.256.5053.70.

KAUPPI, P.E., TOMPPO, E.; FERM, A. 1995. $C$ and $N$ storage in living trees within Finland since 1950s." Plant and Soil, 168-169(1): 633-638. doi: $10.1007 / \mathrm{bf} 00029377$.

KESHWARI, D.R.; CHENG, J.J. 2010. Microwave-based alkali pretreatment of switchgrass and coastal bermudagrass for bioethanol production. Biotechnology Progress, 26: 644-652, 2010.

KHASAN, YY; ZORINA, AD; Matyukhina LG, Saltykova IA. 1977. Triterpenoids of some plants growing in Siberia. Chemistry of Natural Compounds, 13: 109-109.

KIENZE, E.; SCHRAG, I.; BUTTERWICK, R.; OPITZ, B. 2001. Calculation of gross energy in pet foods: new data on heat combustion and fibre analysis in a selection of foods for dogs and cats. J Anim Physiol Anim Nutr, 85: 148-157. doi:10.1046/j.1439-0396.2001.00311.x

KLJUN, A.; BENIANS, T.A.S.; GOUBET, F.; MEULEWAETER, F. KNOX, J.P. BLACKBURN, R.S. 2011. Comparative Analysis of Crystallinity Changes in 
Cellulose I Polymers Using ATR-FTIR, X-ray Diffraction, and CarbohydrateBinding Module Probes. Biomacromolecules, 12: 4121-4126.

KOEHLER, E.S.; WATZLAWICK, F; KIRCHNER, F.F. 2002. Fontes e niveis de erros nas estimativas do potencial de fixação de carbono. En: As florestas e o carbono, edited by C.R. Sanquetta, L.F. Watzlawick, R. Balbinot, M.A.B. Ziliotto and F.S. Gomes, 251-264. Curitiba, Brasil: Imprensa Universitaria da UFPR.

KOLLMANN, F. 1959. Tecnología de la madera y sus aplicaciones. 2 vols. Madrid, España: Instituto Forestal de Investigaciones y Experiencias y Servicios de la Madera. Ministerio de Agricultura.

KOWALSKI, A.S.; SERRANO-ORTIZ, P.; JANSSENS, I.A.; SÁNCHEZ-MORAL, S.; CUEZVA, S.; DOMINGO, F; ALADOS-ARBOLEDAS, L. 2008. Can flux tower research neglect geochemical CO2 exchange? Agricultural and Forest Meteorology, 148(6-7): 1045-1054.

KOWALSKI, A.S., SARTOREM, M.; BURLETT, R.; BERBIGIER, P.; LOUSTAU, D. 2003. The annual carbon budget of a French pine forest (Pinus pinaster) following harvest. Global Change Biology, 9: 1051-1065.

KUMMEROW, J. 1981. Carbon allocation to root systems in Mediterranean evergreen sclerophylls. En: Task for vegetation science, 4: 115-120. N.S. Margaris y N.S. Mooney, eds. W. Junk Publishers, The Hague, Netherlands.

LEE, H.; CHO, D.H.; KIM, Y.H.; SHIN, S.J.; KIM, S.B.; HAN, S.O. et al. 2011. Tolerance of Saccharomyces cerevisiae K35 to lignocellulose-derived inhibitory compounds. Biotechnology and Bioprocess Engineering, 16: 755-760.

LEROY, V.; LEONI, E.; CANCELLIERI, D. 2010. Thermal Degradation of Lignocellulosic Fuels: Biopolymers Contribution. En: Biopolymers, editado por Magdy Elnashar, 303-318. Rijeka, Croatia: InTech.

LEROY, V.; CANCELLIERI, D; LEONI, E. 2006. Thermal degradation of lignocellulosic fuels: DSC and TGA studies. Thermochimica Acta, 451: 131-138

LIN, Y.J.; LIU, C.P.; LIN, J.C. 2002. Measurement of specific gravity and carbon content of important timber species in Taiwan. Taiwan Journal of Forest Science, 17(3): 291-299.

LÖWE, H.; SEUFERT, G.; RAES, F. 2000. Comparison of methods used within Member States for estimating $\mathrm{CO}_{2}$ emissions and sinks according to UNFCCC and EU Monitoring Mechanism: forest and other wooded land. Biotechnologie, agronomie, société et environnement, 4(4): 315-319.

LlORET, F., CASANOVAS, C.; PENUELAS, J. 1999. Seedling survival of Mediterranean shrubland species in relation to root-shoot ratio, seed size and water and nitrogen use. Functional Ecology, 13(2): 210-216. doi: 10.1046/j.13652435.1999.00309.x.

LLISIA, J.; PEÑUELAS, J. 2000. Seasonal patterns of terpene content and emission from seven Mediterranean woody species in field conditions. American Journal of Botany, 87: 133-140.

LI, Y.; FABIANO-TIXIER, A.S.; CHEMAT, F. 2014. Essential oils: From conventional to green extraction. En: Essential oils as reagents in Green Chemistry, Li Y, Fabiano-Tixier A.-S, Chemat F. (Eds.), Springer International Publishing, New York, NY, USA, 9-20. 
LUNDQUIST, K. Lundquist. 1992. Wood. D. S. Y. Lin and P. E. D. C. W. Dence, Eds., ed: Springer Berlin Heidelberg, 1992, pp. 65-70.

MARTÍN-RAMOS, P.; MARTÍN-GIL， J.; RAMOS-SÁNCHEZ， M.C.; NAVAS.GRACIA, L.M.; HERNÁNDEZ-NAVARRO, S.; MARTÍN-GIL, F.J. 2016. Vibrational and thermal characterization of seeds, pulp, leaves and seed oil of Rosa rubiginosa L. Boletín de la Sociedad Argentina de Botánica, 51: 429-439.

MARQUÉS, E.; PAIVA, J.M.; PINHO, C. 2011. The new Portuguese energy challenge? Pellets from shrubs. En: Roncalli A, Telésforo J (eds) 21st Brazilian Congress of Mechanical Engineering, Natal, RN, Brazil, October 24-28, 20112011. ABCM, p 12

MARTÍNEZ, J.M.; VARELA, M.; ESCALADA, R.; MURILLO, J.M.; GONZÁLEZ, E.; CARRASCO, J.; MANZANARES, P. 2000. Combustion assays of brushwood (Cistus ladanifer) biomass in a BAFB pilot plant. 2000. En: Kyritsis S (ed) 1st World Conference on Biomass for Energy and Industry, Sevilla, Spain, June 5-9, 2000. James \& James Science Publishers Ltd., pp 1987-1990

MARTÍNEZ, A; RODRÍGUEZ, M.E.; YORK, S.W.; PRESTON, J.F.; INGRAM, L.O. 2000. Use of UV absorbance to monitor furans in dilute acid hydrolysates of biomass. Biotechnology Progress, 16: 637-641.

MASALLES, R.M.; VIGO, J. 1987. La successió a les terres mediterrànies: sèries de vegetació. En: Ecosistemes terrestres: la resposta als incendis i a d'altres pertorbacions, edited by J. Terradas, 27-43. Barcelona, Spain: Quaderns d'Ecologia Aplicada

MATHIJSEN, D. 2016 Cellulose as reinforcing material for plastics: an alternative between talcum and glass fiber. Reinforced Plastics, 60: 151-153

MC INNES, K. 2014. Esoterismo floral. Dunken, 592 pp.

MELLO, AAD; NUTTO, L.; WEBER, K.S.; SANQUETTA, C.E.; MATOS J.L.M.D.; BECKER, G. 2012. Individual biomass and carbon equations for Mimosa scabrella Benth. (Bracatinga) in Southern Brazil. Silva Fenn, 46: 333-34

MENDOZA-PONCE, A.; GALICIA, L. 2010. Aboveground and belowground biomass and carbon pools in highland temperate forest landscape in Central Mexico. Forestry 83(5): 497-506. doi: 10.1093/forestry/cpq032

MILLER, G.L. 1959. Use of dinitrosalicylic acid reagent for determination of reducing sugar. Analytical Chemistry, 31: 426-428, 1959.

MOHARAM, M.; ABBAS, L. 2010. A study on the effect of microwave heating on the properties of edible oils using FTIR spectroscopy. African Journal of Microbiology Research, 4, 1921-1927.

MOYA PORTUGUÉZ, M.E.; DURAN, M.; SIBAJA-BALLESTEROS. 1992. Obtención de lignina y celulosa de residuos de maíz. Uniciencia, 9: 45-50.

MORÁN, J.I; ÁlVAREZ, V.A.; CYRAS, V.P.; VAZQUEZ, A. 2008. Extraction of cellulose and preparation of nanocellulose from sisal fibers. Cellulose, 15: 149-159.

MOKANY, K.; RAISON, R.J.; PROKUSHKIN, A.S. 2006. Critical analysis of rootshoot ratios in terrestrial biomes. Global Change Biology, 12(1): 84-96. doi: 10.1111/j.1365-2486.2005.001043.x. 
MONTERO, G.; ALONSO, A.; RUIZ-PEINADO, R. 2002. Cuantificación del potencial de las principales especies forestales españolas para el almacenamiento de carbono a medio y largo plazo. Bosques, sociedad y cambio climático. Universidad Menéndez Pelayo. Santander.

MONTERO, G.; MUÑÓZ, M.; AGUDO, R. 2005a. Cuantificación del CO2 fijado por las principales especies arbóreas de Andalucía. IV congreso forestal español. Zaragoza.

MONTERO, G.; RUIZ-PEINADO, R.; MUÑÓZ, M. 2005b. Producción de biomasa y fijación de CO2 por los bosques españoles. Monografías INIA. vol. 13, Monografías INIA: Serie Forestal. Madrid, Spain: Instituto Nacional de Investigación y Tecnología Agraria y Alimentaría (INIA).

MONTERO, G.; PASALODOS-TATO, M.; LÓPEZ-SENESPLEDA, E.; ONRUBIA; R.; MADRIGAL, G. 2013. Ecuaciones para la estimación de la biomasa en matorrales y arbustedos mediterráneos. $6^{\circ}$ Congreso Forestal Español, VitoriaGasteiz, Spain, June 10-14, 2013.

MONTERO, G., PASALODOS-TATO, M.; MONTOTO, R.; LÓPEZ-SENESPLEDA, E.; ONRUBIA, R.; BRAVO-OVIEDO, A.; RUÍZ-PEINADO, R. 2013. Contenido de Carbono en la biomasa de las principales especies de matorral y arbustedos de España. $6^{\circ}$ Congreso Forestal Español, Vitoria-Gasteiz, Spain, June 10-14, 2013.

MOONEY, C.A.; MANSFIELD, S.D.; BEATSON, R.P.; SADDLER, J.N. 1999. The effect of fiber characteristics on hydrolysis and cellulase accessibility to softwood substrates. Enzyme and Microbial Technology, 25: 644-650.

MOYANO, A.; CHARRO E. 2007. Efecto de la reforestación con Prunus avium sobre el secuestro de carbono tanto por la biomasa como por el suelo. Departamento de Ciencias Agroforestales. Universidad de Valladolid. Reunión de la sociedad española forestal.

NAVARRO, R.M. 2004. Fitomasa aérea en los ecosistemas de matorral en el monte Can Vilallonga (T.M. DE Cassà de la Selva-Girona). Ecología, 18: 99-112.

NOMANBHAY, S. M.; HUSSAIN, R.; PALANISAMY, K. 2013. Microwave-Assisted Alkaline Pretreatment and Microwave Assisted Enzymatic Saccharification of Oil Palm Empty Fruit Bunch Fiber for Enhanced Fermentable Sugar Yield. Journal of Sustainable Bioenergy Systems, 3: 7-17.

NUÑEZ, C.E. 2008. Química de la madera. Celulosa, En: Pulpa y Papel I, PROCYP, Facultad de Ciencias Exactas, Químicas y Naturales, Universidad Nacional de Misiones, Misiones, Argentina, 2008, pp. 57-65.

O'CONNOR, R.T.; DUPRÉ, E.F.; MITCHAM, D. 1958. Applications of infrared absorption spectroscopy to investigations of cotton and modified cottons Part I: physical and crystalline modifications and oxidation. Textile Research Journal, 28: 382-392.

OH, S.Y.; YOO, D.I.; SHIN, Y.; SEO, G. 2005. FTIR analysis of cellulose treated with sodium hydroxide and carbon dioxide. Carbohydr. Res., 340: 417-428.

ORELLANA-RIVADENEYRA， G.; SANDOVAL-SAOLÍS， M.L.; LINARESFLEITES, G.; GARCÍA-CALDERÓN, N.E.; TAMARIZ-FLORES; J.V. 2012. Description of the carbon dynamics in forest soils using a model of reservoirs. Avances en Ciencias e Ingeniería, 3(1): 123-135. 
OSORO, K.; VASALlO, J.M.; CELAYA, R.; MARTÍNEZ, A. 1999. Developing viable systems to manage seminatural vegetation in temperate LFAs in Spain. En: Livestock production in the european less favoured areas, editado por Laker, J.P. y Milne, J.A., Dublín, Irlanda, pp. 133-143.

OWEN, N.L.; THOMAS, D.W. 1989. Infrared studies of "hard" and "soft" woods. Applied Spectroscopy, 43(3): 451-455. doi: 10.1366/0003702894202760.

PANDEI, K. 1999. A study of chemical structure of soft and hardwood and wood polymers by FTIR spectroscopy. J. Appl. Polym. Sci., 71: 1969-1975.

PATON, D.; OSORIO, R.; AZOCAR, P.; BOTE, H.; MATAS, A.; TOVAR, J. 1997. Prediction of browsing biomass of certain shrub species from arid Mediterranean climate of North Chile by multicriteria analysis. Archivos de Zootecnia, 46(175): 225-237.

QUINTELA-SABARIS, C.; GIOVANNI GIUSEPPE, V.; CASTRO, D.; FRAGA, M.I. 2011. Chloroplast DNA phylogeography of the shrub Cistus ladanifer L. (Cistaceae) in the highly diverse Western Mediterranean region. Plant Biology 13(2):391-400

RIVAS MARTÍNEZ, S. 1987. Memoria del mapa de series de vegetación de España 1:400.000. Ministerio de Agricultura, Pesca y Alimentación. Madrid, España, 268 pp.

RUÍZ-PEINADO, R.; MONTERO, G.; DEL RÍO, M. 2012. Biomass models to estimate carbon stocks for hardwood tree species. Forest Systems 21 (1):42-52. doi: 10.5424/fs/2112211-02193.

RUÍZ-PEINADO, R.; DEL RÍO, M.; MONTERO, G. 2011. New models for estimating the carbon sink capacity of Spanish softwood species. Forest Systems, 20(1): 176188.

SANQUETTA, C.R.; CORTE, A.P.D.; BALBINOT, R.; ZILLIOTTO, M.A.B. 2004. Proposta metodológica para quantificação e monitoramento do carbono estocado em florestas plantadas. En: Mercado de carbono: mercado e ciência, edited by C.R. Sanquetta and M.A.B. Zilliotto, 240-255. Curitiba, Brazil: UFPR / ECOPLAN.

SANQUETTA, C.R., CORTE, A.P.D.; DA SILVA, F. 2011. Biomass expansion factor and root-to-shoot ratio for Pinus in Brazil. Carbon Balance and Management, 6(6): 1-8. doi: 10.1186/1750-0680-6-6.

SARKANEN, K.V.; LUDWIG, C.H. 1971. Lignins: occurrence, formation, structure and reactions. New York: Wiley-Interscience, 1971.

SCHENK, H.J.; JACKSON, R.B. 2002. Rooting depths, lateral root spreads and belowground/above-ground allometries of plants in water-limited ecosystems. Journal of Ecology, 90 (3): 480-494. doi: 10.1046/j.1365-2745.2002.00682.x.

SCHWANNINGER, M.; RODRIGUES, C.; PEREIRA, H.; HINTERSYOISSER, B. 2004. Effects of short-time vibratory ball milling on the shape of FT-IR spectra of wood and cellulose. Vibrational Spectroscopy, 36(1): 23-40. doi: 10.1016/j.vibspec.2004.02.003.

SÁEZ，J.L. 2017. http://plantararboles.blogspot.com.es/2012/09/arbustosautoctonosespana.html

SEGAL, L.; CREELY, J.; MARTIN, A.; CONRAD, C. 1959. An empirical method for estimating the degree of crystallinity of native cellulose using the X-ray diffractometer. Textile Research Journal, 29: 786-794. 
SOMOGYI, Z.; CIENCIALA, E.; MÄKIPÄÄ, R.; MUUKKONEN, P.; LEHTONEN, A.; WEISS, P. 2007. Indirect methods of large-scale forest biomass estimation. European Journal of Forest Research, 126(2): 197-207. doi: 10.1007/s10342-0060125-7.

STEVENSON, R.L. 2009. Ballads. Pickard Press, 85 pp.

STEWART, C.M. 1969. The formation and chemical composition of hardwoods. Appita, 22, R32.

SUN, Y.; CHENG, J. 2002. Hydrolysis of lignocellulosic materials for ethanol production: a review. Bioresource technology, 83: 1-11.

SUN, S.; SONG, Y.; SUN, P.; CHEN, J. 2013. Furfural, acid-soluble lignin and reducing sugar content in biomass hydrolysate by spectrophotometric method. Acta Energiae Solaris Sinica, 34: 331-336.

TALWALTAR, A.T. 1981. United S, Department of Energy, Institute of Gas T. IGT/DOE coal-conversion systems technical data book. Institute of Gas Technology, Chicago, Ill.

TERRAB, A.; ANDRÉS, C.; DÍEZ, M. J. 2004. Análisis polínico de mieles en el Parque Natural Sierra de Aracena y Picos de Aroche. Botanica Complutensis, 28:121-126.

TERRADAS, J. 2001. Ecología de la vegetación: de la ecofisiología de las plantas a la dinámica de comunidades y paisajes. Barcelona, Spain: Omega.

THOMSON REUTERS. 2014. The World in 2025: 10 predictions of innovation. T.R.I. Science, ScienceWatch.com, pp. 28.

TIHAY, V. SANTONI, P.A.; SIMEONI, A.; GARO, J.P.; VANTELON, J.P. 2009. Skeletal and global mechanisms for the combustion of gases released by crushed forest fuels. Combust Flame, 156: 1565-1575. doi:10.1016/j.combustflame.2009.05.004.

TRAORÉ, M.; KAAL, J.; MARTÍNEZ CORTIZAS, A. 2016. Application of FTIR spectroscopy to the characterization of archeological wood. Spectrochimica Acta Part A: Molecular and Biomolecular Spectroscopy, 153: 63-70. doi: 10.1016/j.saa.2015.07.108.

UN-ECE/FAO. 2000. Forest resources of Europe, CIS, North America, Australia, Japan, and New Zealand (industrialized temperate/boreal countries): UNECE/FAO contribution to the Global Forest Resources Assessment 2000. Vol. 17, Geneva timber and forest study papers. New York, USA: United Nations, Economic Commission for Europe, Timber Section; Food and Agriculture Organization of the United Nations.

VALARES-MASA, C.; SOSA-DÍAZ, T.; ALÍAS-GALLEGO, J.; CHAVES-LOBÓN, N. 2016. Quantitative variation of flavonoids and diterpenes in leaves and stems of Cistus ladanifer L. at different ages Molecules, 21: 275-288. doi:10.3390/molecules21030275.

VARTANIAN, E.; BARRES, O.; ROQUE, C. 2015. FTIR spectroscopy of woods: A new approach to study the weathering of the carving face of a sculpture. Spectrochimica Acta - Part A: Molecular and Biomolecular Spectroscopy, 136: 1255-1259. doi: 10.1016/j.saa.2014.10.011 
VERDEGUER, M.; BLAZQUEZ, M.A.; BOIRA, H. 2012. Chemical composition and herbicidal activity of the essential oil from a Cistus ladanifer L. population from Spain. Natural Product Research, 26: 1602-1609.

VEGA-BAUDRIT, J.; DELGADO-MONTERO, K.; SIBAJA-BALLESTERO, M.; ALVARADO-AGUILAR, P. 2007. Uso alternativo de la melaza de la caña de azúcar residual para síntesis de espuma rígidas de poliuretano (ERP) de uso industrial. Tecnología, Ciencia, Educación, 22: 101-107.

VLACHOS, N.; SKOPELITIS, Y.; PSAROUDAKI, M.; KONSTANTINIDOU, V.; CHATZILAZAROU, A.; TEGOU, E. 2006. Applications of Fourier transforminfrared spectroscopy to edible oils. Analytica Chimica Acta, 573: 459-465.

VV.AA. 2013. Elaboración del Diagnóstico sectorial de los territorios del proyecto de cooperación, Red de Territorios Sostenibles, R.E.T.O.S. (2013), Macovall (2000, ríos llenos de vida) y Ministerio de Agricultura, Alimentación y Medio Ambiente.

WANG, F et al. 2016. Regulation for Optimal Liquid Products during Biomass Pyrolysis: A Review IOP Conference Series: Earth and Environmental Science, 40: 012047. doi:10.1088/1755-1315/40/1/012047

WANG, Y.; CAO, X.; ZHANG, L. 2006. Effects of Cellulose Whiskers on Properties of Soy Protein Thermoplastics, Macromol. Biosci, 6: 524-531.

WANG, J.; DUAN, B.; ZHANG, Y. 2011. Effects of experimental warming on growth, biomass allocation, and needle chemistry of Abies faxoniana in even-aged monospecific stands. Plant Ecology, 213(1): 47-55. doi: 10.1007/s11258-011-00051.

WATSON, R.T.; NOBLE, I.R. 2002. Carbon and the Science-Policy Nexus: The Kyoto Challenge. En: Challenges of a Changing Earth, edited by Will Steffen, Jill Jäger, David J. Carson and Clare Bradshaw, 57-64. Berlin, Heidelberg: Springer Berlin Heidelberg.

ZABANIOTOU, A.A.; ROUSSOS, A.I.; KORONEOS, C.J. 2000. A laboratory study of cotton gin waste pyrolysis. J Anal Appl Pyrolysis, 56: 47-59. doi:10.1016/s01652370(00)00088-7

ZU, Z.; WEN, Z. 2008. Enhancing enzymatic digestibility of switchgrass by microwaveassisted alkali pretreatment. Biochemical Engineering Journal, 38: 369-378. 
